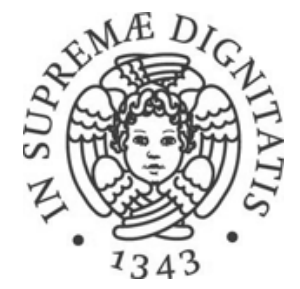

UNIVERSITY OF PISA

Department of Computer Science

Master Degree Program in Computer Science

MASTER THESIS

\title{
Matching cloud services with TOSCA
}

SUPERVISOR

Prof. Antonio BROGI

CANDIDATE

Jacopo SOLDANI

Academic Year 2012-2013 
To those who made this possible, both who can be here and understand and who cannot. 


\begin{abstract}
The OASIS TOSCA specification aims at enhancing the portability of cloud-based applications by defining a language to describe and manage service orchestrations across heterogeneous clouds. A service template is defined as an orchestration of typed nodes, which can be instantiated by matching other service templates. In this thesis, after defining the notion of exact matching between TOSCA service templates and node types, we define three other types of matching (plugin, flexible and white-box), each permitting to ignore larger sets of non-relevant syntactic differences when type-checking service templates with respect to node types. We also describe how service templates that plug-in, flexibly or whitebox match node types can be suitably adapted so as to exactly match them.
\end{abstract}




\section{Contents}

$\begin{array}{lll}1 & \text { Introduction } & 1\end{array}$

$\begin{array}{lll}2 & \text { Background: TOSCA } & 6\end{array}$

2.1 Use cases of TOSCA $\ldots \ldots \ldots \ldots \ldots$

2.2 TOSCA syntax $\ldots \ldots \ldots \ldots \ldots$

2.3 A TOSCA example . . . . . . . . . . . . . . 21

$3 \quad$ Node Type black-box matching (and adaptation) 30

$3.1 \quad$ Exact matching . . . . . . . . . . . . . . . 31

3.1.1 Definition of exact matching . . . . . . . . 31

3.1 .2 Exact matching examples . . . . . . . . 44

$3.2 \quad$ Plug-in matching . . . . . . . . . . . . . . . 47

3.2.1 Definition of plug-in matching . . . . . . . 47

3.2.2 Adaptation: the oblivion boundaries approach . . 52

3.2.3 $\quad$ Plug-in matching (and adaptation) examples . . . 56

$3.3 \quad$ Flexible matching $\ldots \ldots \ldots \ldots$. . . . . . . . . . 61

3.3.1 Definition of flexible matching . . . . . . . . 62 
3.3.2 Adaptation: extended oblivion boundaries approach 66

3.3.3 $\quad$ Flexible matching (and adaptation) examples . . 66

3.4 Concluding remarks $\ldots \ldots \ldots \ldots \ldots$. . . . . . . . 67

3.4.1 Matching and adaptation . . . . . . . . . 67

3.4 .2 Taking semantics into account . . . . . . . 70

3.4.3 Treatment of mismatchings . . . . . . . . 70

$4 \quad$ Node Type white-box matching (and adaptation) 72

4.1 Motivating example . . . . . . . . . . . . . 72

4.2 White-box matching (and adaptation) . . . . . . 74

4.2.1 White-box matching condition . . . . . . . 75

4.2 .2 Adaptation . . . . . . . . . . . 80

4.3 Generating plans . . . . . . . . . . . . . 82

$4.3 .1 \quad$ Functional dependency synthesis . . . . . . 83

4.3 .2 Operation sequence detection . . . . . . . 88

4.3 .3 Example . . . . . . . . . . . . . . 103

4.4 Concluding remarks $\ldots \ldots \ldots \ldots \ldots$. . . . . . . . 110

$4.4 .1 \quad$ A complete example . . . . . . . . . . . . 110

$4.4 .2 \quad$ Matching and adaptation . . . . . . . . . . 113

4.4 .3 Taking semantics into account . . . . . . . . 115

4.4 .4 Practical observations . . . . . . . . . . 116

4.4 .5 What's next? . . . . . . . . . . . 116

$\begin{array}{lll}5 & \text { Proof-of-concept implementation } & 118\end{array}$ 
$5.1 \quad$ Implementation of basic features . . . . . . . . . . . . 119

5.2 Implementation of the needed TOSCA components . . . 132

5.3 Implementation of the matchmakers . . . . . . . . . . 135

5.4 Concluding remarks . . . . . . . . . . . . . 154

$\begin{array}{lll}6 & \text { Conclusions } & 155\end{array}$

6.1 Summary of contributions . . . . . . . . . . . 155

6.2 Related work . . . . . . . . . . . . . . 157

6.3 Future work . . . . . . . . . . . . . . . . . . 159

A Example of test of the proof-of-concept implementation 161

A.1 Implementation of the needed input . . . . . . . . . 163

A.2 Implementation of the Test . . . . . . . . . 175

A.3 Concluding remarks . . . . . . . . . . . . . . . 177 


\section{List of Figures}

$2.1 \quad$ TOSCA Service template $[25] . \ldots \ldots \ldots \ldots$

2.2 Requirement and capabilities [25] . . . . . . . . 18

$2.3 \quad$ TOSCA cloud application example. . . . . . . . . 21

$3.1 \quad$ Service Template substituting a NodeType [26]. . . . . . . 31

3.2 TOSCA DBMSNodeType example. . . . . . . . . . . 44

$3.3 \quad$ TOSCA Service Template exact matching example. . . . . 45

$3.4 \quad$ TOSCA ServiceTemplate exact matching example (modified). ................... 46

$3.5 \quad$ Oblivion boundaries adaptation approach. . . . . . . 53

$3.6 \quad$ Echo node usage in the oblivion boundaries adaptation approach. .................. 55

3.7 TOSCA Service Template plug-in matching example. . . 58

3.8 TOSCA ServiceTemplate oblivion boundaries adaptation example. . . . . . . . . . . . 59

3.9 TOSCA Service Template plug-in matching example (modified). . . . . . . . . . . . 6 6 60

3.10 Black-box matching (and adaptation) procedure. . . . . 68 
3.11 Adaptation approach. . . . . . . . . . . . . 69

4.1 Example of available ServiceTemplate. . . . . . . 73

$4.2 \quad$ Example of desired NodeType elements. . . . . . . . . 73

$4.3 \quad$ A directed hyperedge. . . . . . . . . . . . . 84

4.4 OPERATIONSETSDISCOVERING algorithm. . . . . . . . . 91

$4.5 \quad$ Parameters ontology example. . . . . . . . . . . . . 104

$4.6 \quad$ Dependency hypergraph example. . . . . . . . . . . . 104

4.7 Example of generated plan. . . . . . . . . . . . 109

$4.8 \quad$ A complete matching example. . . . . . . . . . 111

4.9 Complete matching example adaptation. . . . . . . . 113

4.10 Extended matching (and adaptation) procedure. . . . . . 114

$5.1 \quad$ UML diagram of the developed matchmakers. . . . . . 136

6.1 Complete matching (and adaptation) procedure. . . . . . 160

A.1 Node type and service templates employed in testing the proof-of-concept implementation. . . . . . . . . . . . . . 162

A.2 Test results. . . . . . . . . . . . . . . . . . 177 


\section{List of Listings}

$2.1 \quad$ TOSCA Definitions element high level syntax . . . . . . 10

2.2 TOSCA Service Template element high level syntax . . . 12

2.3 TOSCA NodeType element high level syntax. . . . . . . . 14

2.4 TOSCA Relationship Type element high level syntax. . . . 16

2.5 TOSCA RequirementType element and CapabilityType element high level syntax. . . . . . . . . . . 17

$2.6 \quad$ TOSCA Policy Type element high level syntax . . . . . . 19

2.7 TOSCA Policy Template element high level syntax. . . . 20

2.8 TOSCA example Definitions document. . . . . . . . . 21

2.9 TOSCA example requirement and capability types definitions. . . . . . . . . . . . . . . . . 22

2.10 Properties structure definitions required in our TOSCA example. . . . . . . . . . . . 23

2.11 TOSCA example NodeType definitions. . . . . . . . . 23

2.12 TOSCA example Relationship Type definition. . . . . . . 25

2.13 TOSCA example Topology Template definition. . . . . . . 26

2.14 TOSCA example Plans definition. . . . . . . . . . 27

2.15 TOSCA example boundary definitions. . . . . . . . 28 
$3.1 \quad$ High level syntax of TOSCA NodeType and ServiceTemplate elements interested in requirements matching. . . . 32

3.2 High level syntax of TOSCA elements interested in policies matching. . . . . . . . . . . . . 35

3.3 High level syntax of TOSCA NodeType and ServiceTemplate elements interested in properties matching. . . . . . 37

3.4 High level syntax of TOSCA NodeType and ServiceTemplate elements interested in interfaces matching. . . . . . 40

$5.1 \quad$ JAVA implementation of a generic capability (type). . . 119

$5.2 \quad$ JAVA implementation of a generic requirement (type). . 120

$5.3 \quad$ JAVA implementation of a generic policy (type). . . . . . 121

$5.4 \quad$ JAVA implementation of a set of policies. . . . . . . . 122

$5.5 \quad$ JAVA implementation of a property. . . . . . . . . 124

$5.6 \quad$ JAVA implementation of an operation parameter. . . . . 125

5.7 JAVA implementation of an operation parameter. . . . . 127

$5.8 \quad$ JAVA implementation of an interface. . . . . . . . . . . 129

$5.9 \quad$ JAVA implementation of a generic service component. . . 132

5.10 JAVA implementation of a generic NodeType. . . . . . 134

5.11 JAVA implementation of a generic Service. . . . . . . . . 134

5.12 JAVA implementation of the abstract Matchmaker. . . 135

5.13 JAVA implementation of the ExactMatchmaker (1). . . . 139

5.14 JAVA implementation of the ExactMatchmaker (2). . . . 142

5.15 JAVA implementation of the PlugInMatchmaker (1). . . 146 
5.16 JAVA implementation of the PlugInMatchmaker (2). . . 149

A.1 JAVA implementation of the CalculatorCapabilityType. 163

A.2 JAVA implementation of the ExtendedCalculatorCapabilityType................. 163

A.3 JAVA implementation of the RapidCalculatorPolicyType. 164

A.4 JAVA implementation of the CalculatorNodeType. . . . 164

A.5 JAVA implementation of Service. . . . . . . . . 166

A.6 JAVA implementation of ServiceBis. . . . . . . . 169

A.7 JAVA implementation of ServiceTer. . . . . . . . . 172

A.8 JAVA implementation of the Test. . . . . . . . . 175 


\section{Chapter 1}

\section{Introduction}

How to deploy and manage, in an efficient and adaptive way, complex multi-service applications across heterogeneous cloud environments is one of the problems that have emerged with the cloud revolution [30]. Currently, migrating (parts of) an application from one cloud to another is a costly and error-prone process that has to be performed manually. Part of (if not the whole) application must be stopped in order to migrate services (possibly along with data) to a different cloud and to restart them, taking care of synchronizing and maintaining the interoperability with the rest of the application. As a result, cloud users tend to end up locked into the cloud platform they are using since it is practically unfeasible for them to migrate (parts of) their application across different clouds platforms [29].

In this scenario, OASIS recently created a Technical Committee on Topology and Orchestration Specification for Cloud Application (TOSCA), whose goal is to ease the portability of cloud-based applications by defining a language to describe and manage service orchestrations across heterogeneous clouds. The first specification of TOSCA [25] defines a XML-based lanugage that permits to specify (in a vendor-agnostic way) topology and behaviour of complex multi-cloud applications as service 
templates that orchestrate typed nodes.

The expected impact of TOSCA on cloud service portability is well explained in [18]. Let us think of similar attempts to deal with the lifecycle of complex man-made structures (like skyscrapers or bridges). Such structures are constructed, modified, maintained, and even dismantled using industry standard descriptions and manifests. The focus of these documents is primarily at the conceptual level of the building itself, its principal components, construction and maintenance, and not on how those components (like a furnace or elevator) are actually built themselves. Similarly, TOSCA is designed to support the definition of a common, machine-readable language for service description, maintenance, and operational management (which are the best practices needed to support cloud services across their lifetime). In other words, TOSCA enables the creation of an eco-system in which cloud service developers can describe (and model) the principal components, characteristics and requirements of a service in a standardized fashion so that the service can be understood, installed (deployed) or removed (undeployed) by different types of TOSCA-compliant cloud providers with very little additional effort.

As stated in the TOSCA primer [26], node types can be made concrete by substituting them by a service template. However, while the matching between service templates and node types is mentioned with reference to an example ([26], page 35$)$ :

«Service template ST may substitute node type $N$ because the boundary of ST matches all defining elements of $N$ : all properties, operations, requirements and capabilities of ST match exactly those of $N . »$,

no formal definition of matching is given either in [25] or in [26]. A definition of matching is employed in [33] to merge TOSCA services by matching entire portions of their topology templates. The definition of 
matching employed in [33] is however very strict, as two service components are considered to match only if they expose the same qualified name.

The objective of this work is to contribute to the TOSCA specification by first providing a formal definition of the notion of exact matching between TOSCA ServiceTemplate and NodeType elements, and by then extending such definition in order to provide three other types of matching (plug-in, flexible and white-box), each permitting to ignore larger sets of non-relevant syntactic differences when type-checking service templates with respect to node types. More precisely:

- the plug-in matching extends the exact one by considering a service that "require less" and "offers more" than a node type compatible with the latter;

- the flexible matching in turn extends the plug-in one by employing ontologies to check whether differently named features are semantically equivalent (so as to ignore non-relevant syntactic differences);

- the white-box matching in turn extends the flexible one by searching missing (equivalent) features inside the service topology. It still employs ontologies to check whether differently named features can be considered semantically equivalent. Furthermore, it employs a recursive algorithm to detect available compositions of operations which are semantically equivalent to needed (missing) operations.

To allow exploiting the new notions of matching not only during typechecking but also for node instantiation, we describe how a service template that plug-in, flexibly or white-box matches a typed node can be suitably adapted so as to exactly match it.

The results presented in this thesis intend to contribute to the formal definition of TOSCA. The different types of matching defined in this thesis can be fruitfully integrated in the TOSCA implementations that are 
currently under development (such as [27] and [32]) in order to enhance their type-checking capabilities. More in general, the presented definitions of matching can be exploited to implement type-checking mechanism over service descriptions by taking into account, beyond functional features, also requirements, capabilities, policies, and properties.

It is worth pointing that implementing our matching notions (e.g., as a plug-in of TOSCA IDEs) will contribute to cloud service portability and multi-cloud service development. Indeed, with the availability of such an implementation, a cloud service developer will have the possibility to:

- employ more available (adapted) cloud services instead of developing her application's encompassed components,

- migrating more application's components across heterogeneous clouds by changing the used available (adapted) cloud services, and

- choose between more different cloud service providers the one which provides the compatible service with the best quality-price ratio.

\section{Outline}

The rest of the document is organized as follows:

Chapter 2 provides an overview of the TOSCA specification [25].

Chapter 3 starts by formalizing the TOSCA [25] notion of (black-box) exact matching. It then proceeds showing two other ways to match services from a black-box viewpoint (plug-in, flexible). Along with these matching notions, a way to adapt available service templates (in order to make them exactly match the desired node types) is provided. 
Chapter 4 extends the matching notion of Chapter 3 by moving the viewpoint to a white-box one. A way to adapt service templates which white-box match the desired node types is given too.

Chapter 5 shows a partial implementation of the matching procedure in order to demonstrate the feasibility of the notions proposed in this thesis.

Chapter 6 summarizes the contributions of this thesis, discusses the work in related research fields and provides some concluding remarks. 


\section{Chapter 2}

\section{Background: TOSCA}

The Topology and Orchestration Specification for Cloud Application (TOSCA) [25] is an XML-based language and metamodel which can be used to describe IT services. The main goal of the TOSCA specification is to allow a description of composite cloud-based applications and of their management in a modular and portable fashion.

The creator of a cloud service defines it in a so-called service template (Figure 2.1). This template is composed by:

- a topology template, a graph in which typed nodes represent service's components and typed relationships connect and structure nodes into the topology, and by

- plans, workflows used to describe managing concerns.

The following sections describe the most important elements of the TOSCA specification [25]. Before describing the TOSCA syntax (Section 2.2 , we proceed by showing the major use cases supported by this specification (Section 2.1). Once all the basic concepts are given, we provide a complete TOSCA cloud service example (Section 2.3). 


\section{Service Template}

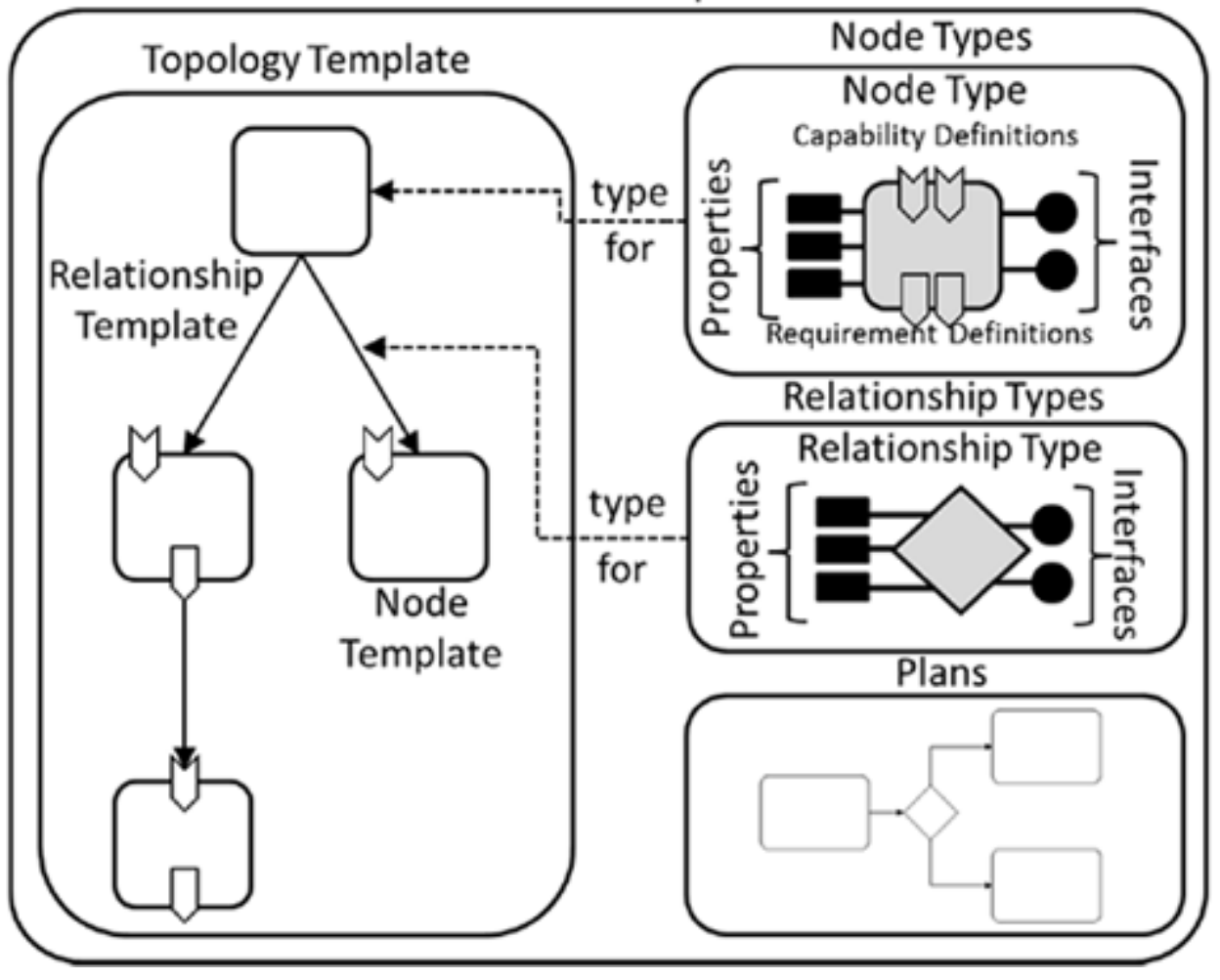

Figure 2.1: TOSCA Service template [25].

\section{$2.1 \quad$ Use cases of TOSCA}

In the previous paragraph we have seen which is the main use case of TOSCA: to provide a standard support in specifying topological and management aspects of cloud service applications. Despite this, in the TOSCA specification [25] several other supported use cases are proposed.

\section{Service as marketable entities}

According to the authors, the service template standardization will cause the creation and spread of a market for hosted IT services.

Having a standard for topology template definition enables interoperable specification of services' structure. Especially, that standard will let 
cloud service development experts define service topology models. Those models could then be published in repositories of one or more service providers. Each provider would map the specified service topology to its available concrete infrastructure in order to support concrete instances of the service and adapt the management plans accordingly.

Furthermore, making a concrete instance of a topology template can be done by running a corresponding plan (also known as build plan). So, the service developer who creates the service template could provide this plan too. The build plan can be adapted to the concrete environment of a particular service provider. This is not only the case of build plans: other management plans of a service could then be specified as part of a service template (and adapted to the providers' infrastructure).

Thus, not only the structure of a service can be defined in an interoperable manner, but also its management plans. Such a configuration will let consumers easily search, select and use predefined IT services (hosted on cloud service providers).

\section{Portability of service templates}

TOSCA service templates standardization will support the portability of IT service definitions. Observe that, as specified by the TOSCA authors, portability denotes the ability of one cloud provider to understand the structure and behaviour of a service template created by another party (e.g., another cloud provider, enterprise IT department, service developer).

Furthermore, if a service template is portable, this does not mean that its encompassed components are portable too. Portability of a service only implies that its definition is comprehensible in an interoperable manner (i.e., the topology model and corresponding plans are understood by TOSCA-compliant providers). Individual components portability has to be ensured (if needed) via other mechanisms. 


\section{Service composition}

Another use case specified by TOSCA authors is the composition of service templates. Since a service template provides an abstraction that does not make assumption about the hosting environment, the deployed service could be hosted on more than one cloud provider. This enables an important feature: multi-cloud service applications deployment (e.g., large organization could use automation products from different suppliers for different data centers).

\subsection{TOSCA syntax}

In this section we will show how cloud services can be defined using TOSCA. To do it, we will follow the same notation of the TOSCA specification to define the serialization of resources:

- characters are appended to items to indicate cardinality:

$$
\begin{aligned}
& \text { - "?" }(0 \text { or } 1) ; \\
& \text { - "*" ( } 0 \text { or more }) ; \\
& \text { - "+" (1 or more })
\end{aligned}
$$

- vertical bars, "I", denote choice;

- parentheses, "(" and ")", are used to indicate the scope of the operators "?", "*", "+" and "I";

- ellipses (i.e., "...") indicate points of extensibility.

\section{Definitions}

All elements needed to define a cloud service are provided in the TOSCA Definitions element (Listing 2.1). This element is the root of a TOSCA 
XML document. It has a set of properties; the most important ones will be discussed next.

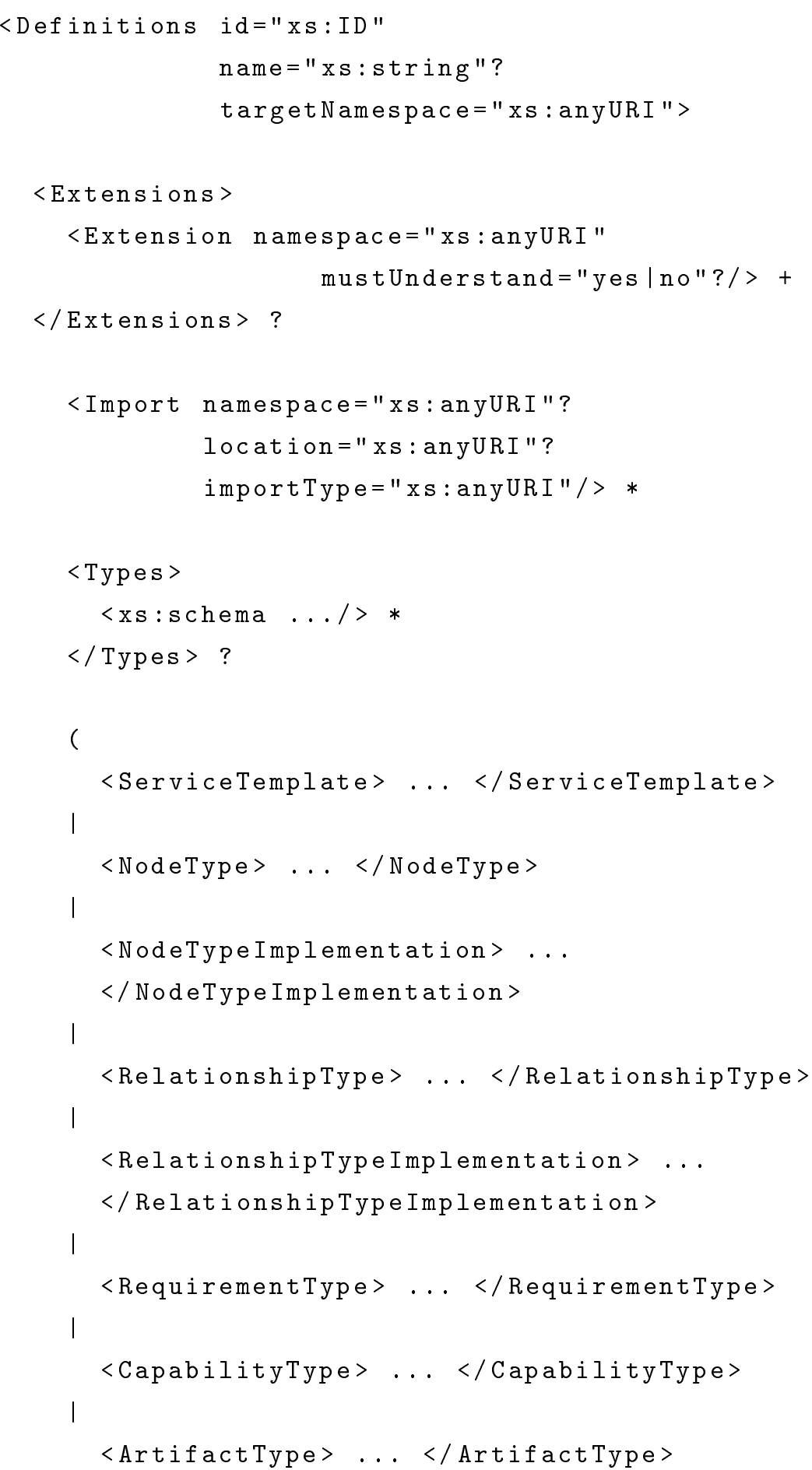




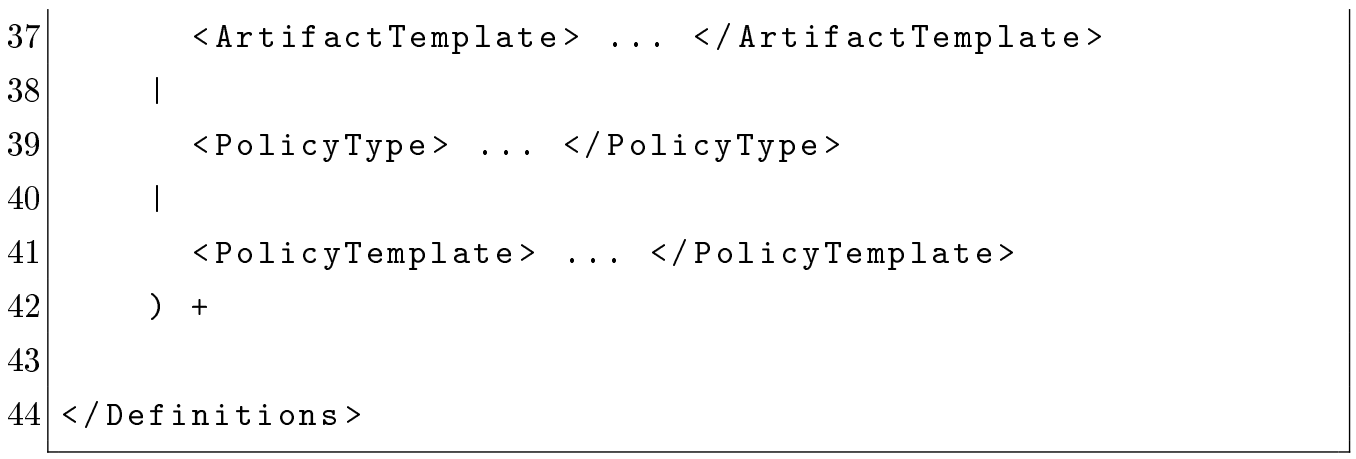

Listing 2.1: TOSCA Definitions element high level syntax

Each Definitions element has a unique $i d$ regarding its namespace and (possibly) a descriptive, human-readable name. In addition, the targetNamespace attribute declares the namespace of the Definitions. This is an important feature because it lets other Definitions elements reference this one.

The optional Import element provides means to use external Definitions, XML Schemas or WSDL definitions. A Definitions element must name all external references that it uses via Import elements.

With the optional Types element the application developer can specify additional XML definitions to use throughout the Definitions document (e.g., as attributes in other elements). In this way, the developer does not need to define them in separate documents and import them via Import elements. The types are XML Schema elements by default but they could also be of arbitrary types.

The explanation of the other elements reported in Listing 2.1 is given in the following sections.

\section{Service templates}

The ServiceTemplate element (Listing 2.2) specifies each topological and management aspect of a cloud application by means of TopologyTemplate elements and Plans, respectively. 


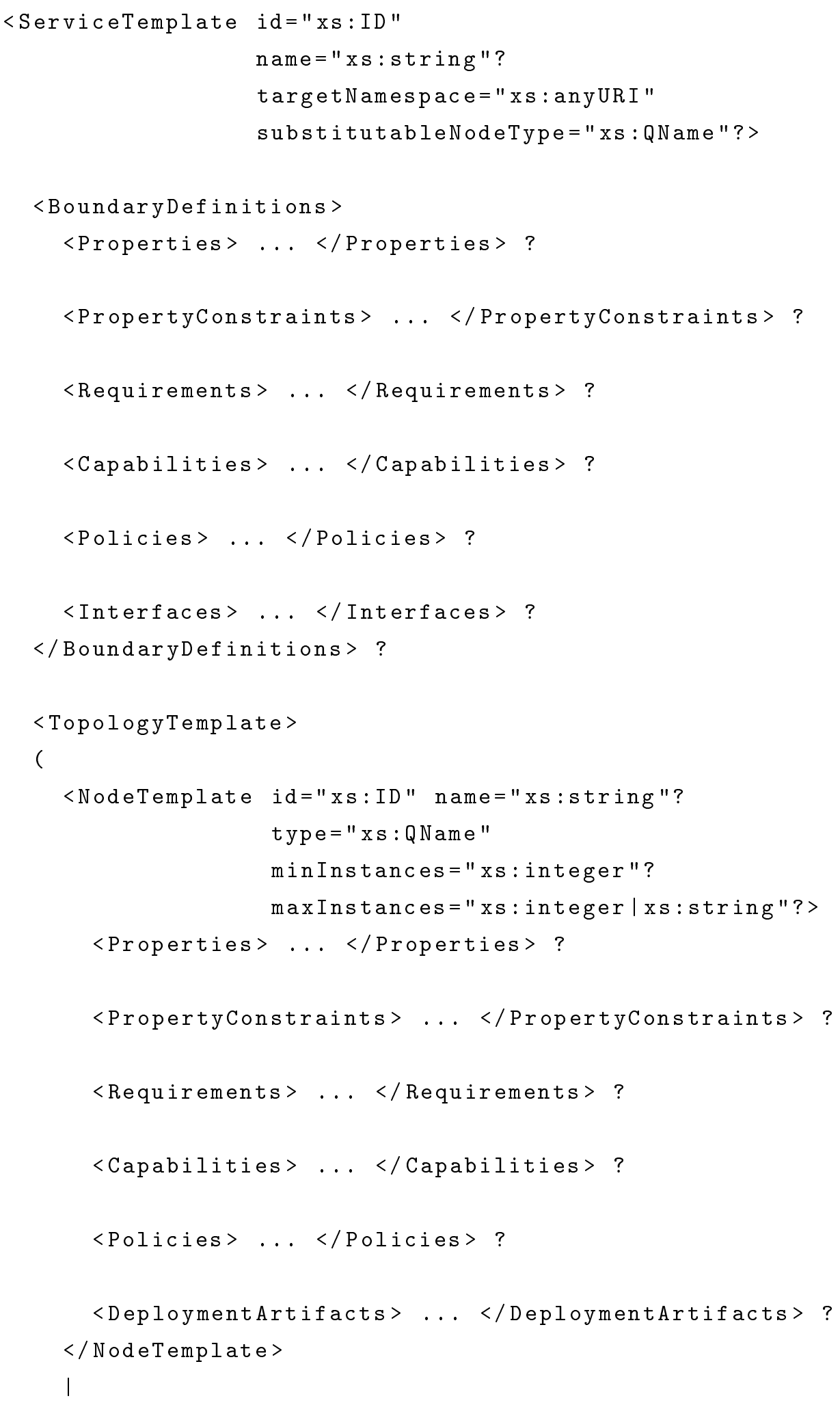




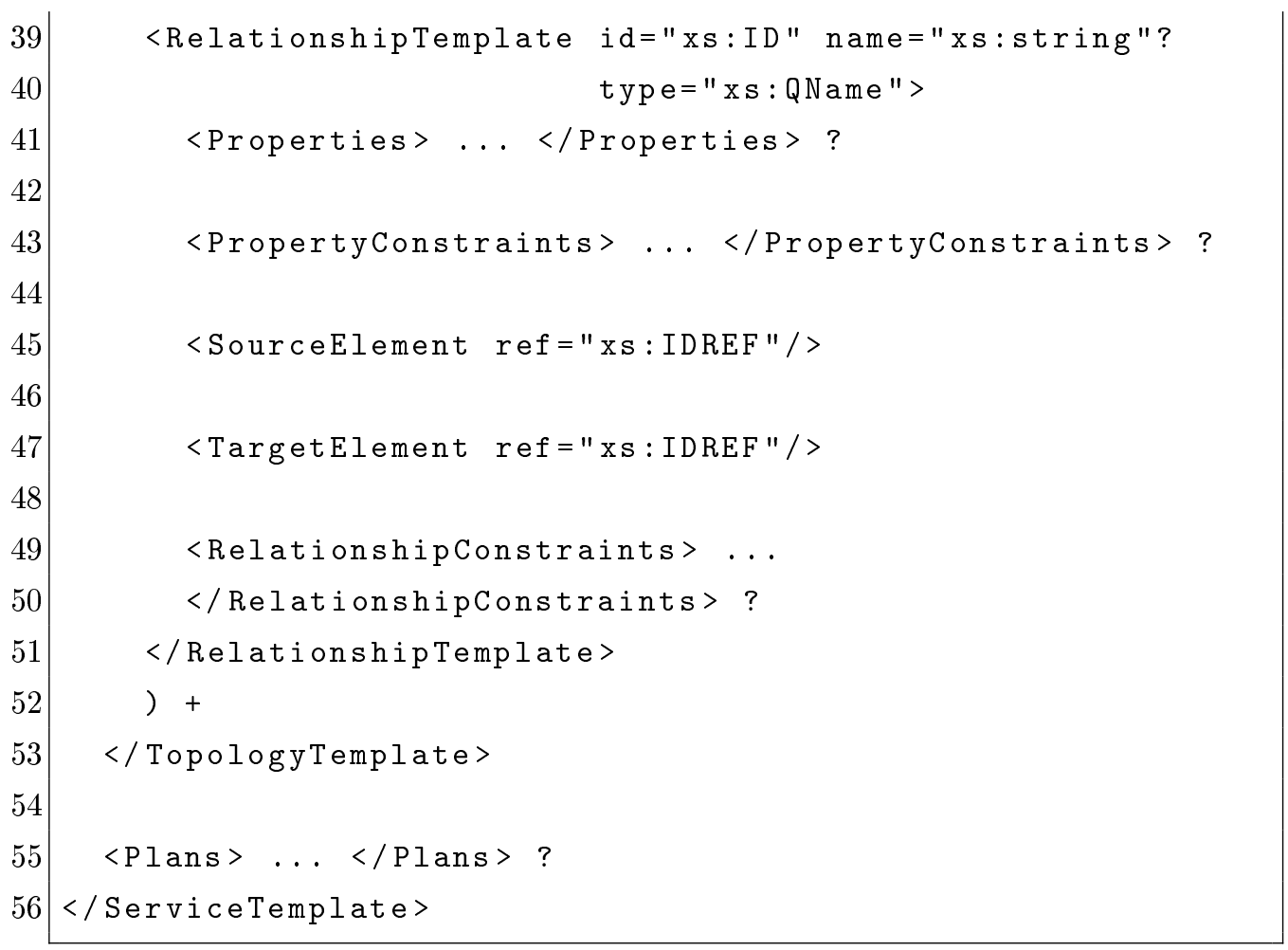

Listing 2.2: TOSCA ServiceTemplate element high level syntax

As for the Definitions element, each ServiceTemplate element requires an unique $i d$ in its own targetNamespace. An important additional attribute is substitutableNodeType. This attribute specifies the NodeType that can be substituted by this ServiceTemplate. So, if another ServiceTemplate contains a NodeTemplate of the specified NodeType (or any NodeType which is derived from the specified one), then such NodeTemplate can be substituted by an instance of the ServiceTemplate under definition.

Another way a node type can be substituted by a service template is by matching what it exposes (Properties, Requirements, Capabilities, Policies and Interfaces) with what a ServiceTemplate exposes. The latter comes with the BoundaryDefinitions element.

When the cloud application developer has specified all the global aspects, she could proceed in defining the topology and management concerns. 
Topology template A TopologyTemplate defines the topological structure of an IT service as a directed graph. The vertices are represented by a set of NodeTemplate elements and the directed edges by a set of Relationship Template elements 1 . Each edge expresses the semantics of the relationship between nodes.

Plans The Plans element contains Plan elements specifying how to manage the ServiceTemplate under definition during its life cycle (e.g., how to deploy, start and stop it).

\section{Node types}

With the Node Type element (Listing 2.3) it is possible to specify a reusable entity that defines the type of one or more node templates in a ServiceTemplate element.

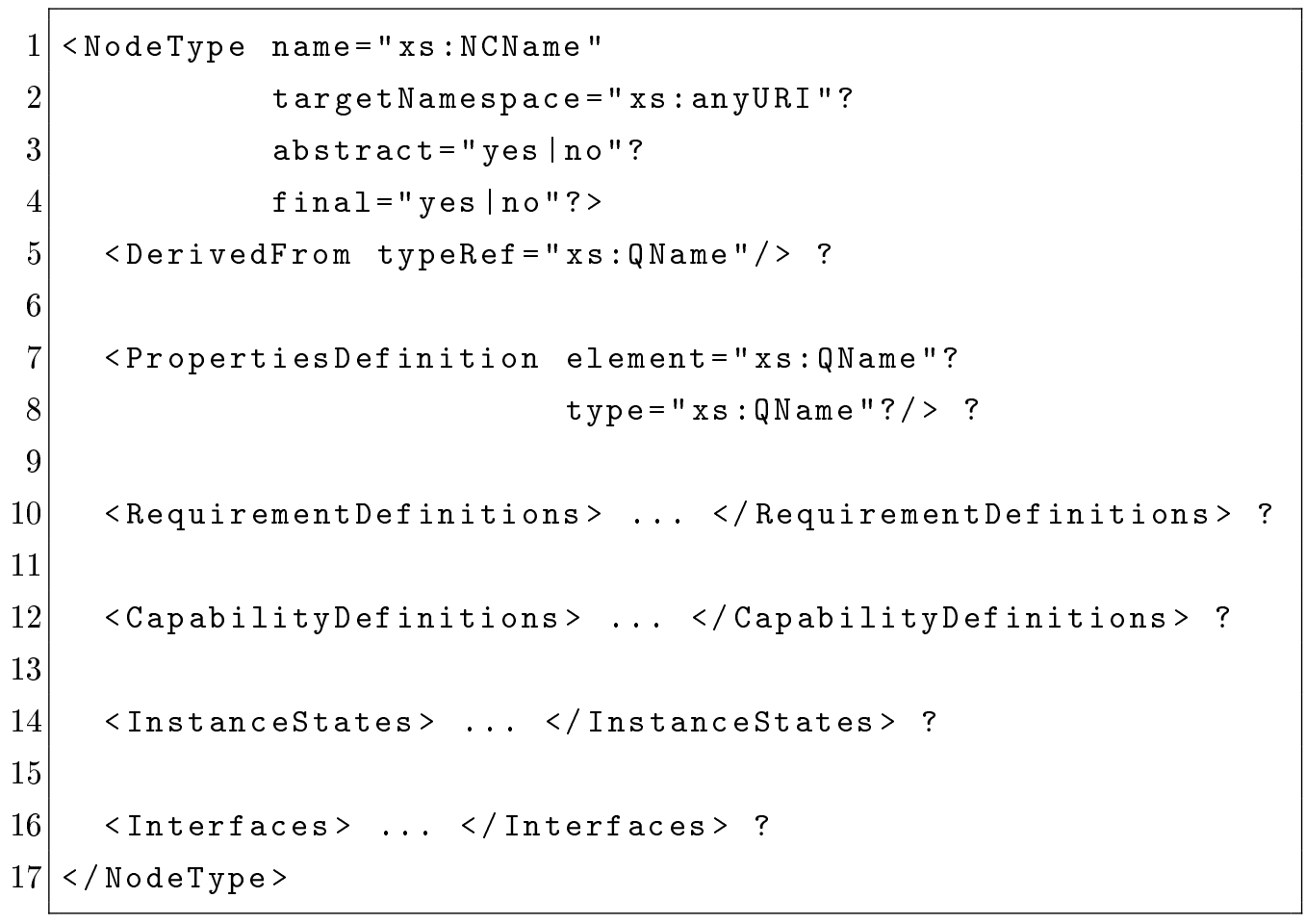

Listing 2.3: TOSCA NodeType element high level syntax.

\footnotetext{
${ }^{1}$ Both NodeTemplate elements and RelationshipTemplate elements are typed by referring NodeType elements and Relationship Type elements, respectively.
} 
Each NodeType requires a unique name to be identified in its own targetNamespace. The NodeType under definition could be also specified as abstract or final (if needed). The former means that no instances can be created from NodeTemplate elements that use this NodeType as their type. The latter says that other NodeType elements must not be derived from the under definition one.

With the optional DerivedFrom element, the node type developer can implement inheritance. Conflicting definitions are resolved by the rule that local new definition always override derived definitions.

The optional RequirementDefinitions and CapabilityDefinitions elements are used to specify which properties and capabilities the NodeType under consideration exposes.

The InstanceStates element is used to model the life cycle of an instance of this NodeType. Indeed, this element specifies the set of states an instance of this NodeType can occupy.

Finally, with the optional element Interfaces the developer could define the operations which can be performed on (instances of) this NodeType. Such operations definition is given in the form of nested Interface elements (each of which is characterized by a name and some Operation elements).

Once the NodeType has been defined, it should be linked with the executables by which is implemented. NodeTypeImplementation elements provide a collection of implementation artifacts (executables implementing the interface operations) and deployment artifacts (executables needed to materialize instances of NodeTemplate elements referring the NodeType under consideration) ${ }^{2}$.

\footnotetext{
${ }^{2}$ The respective executables are defined as separate ArtifactTemplate elements and are referenced from the implementation artifacts and deployment artifacts of a NodeTypeImplementation.
} 


\section{Relationship types}

As mentioned earlier, pairs of NodeTemplate elements are connected by Relationship Template elements. Each of these Relationship Template elements is typed by a Relationship Type element (Listing 2.4).

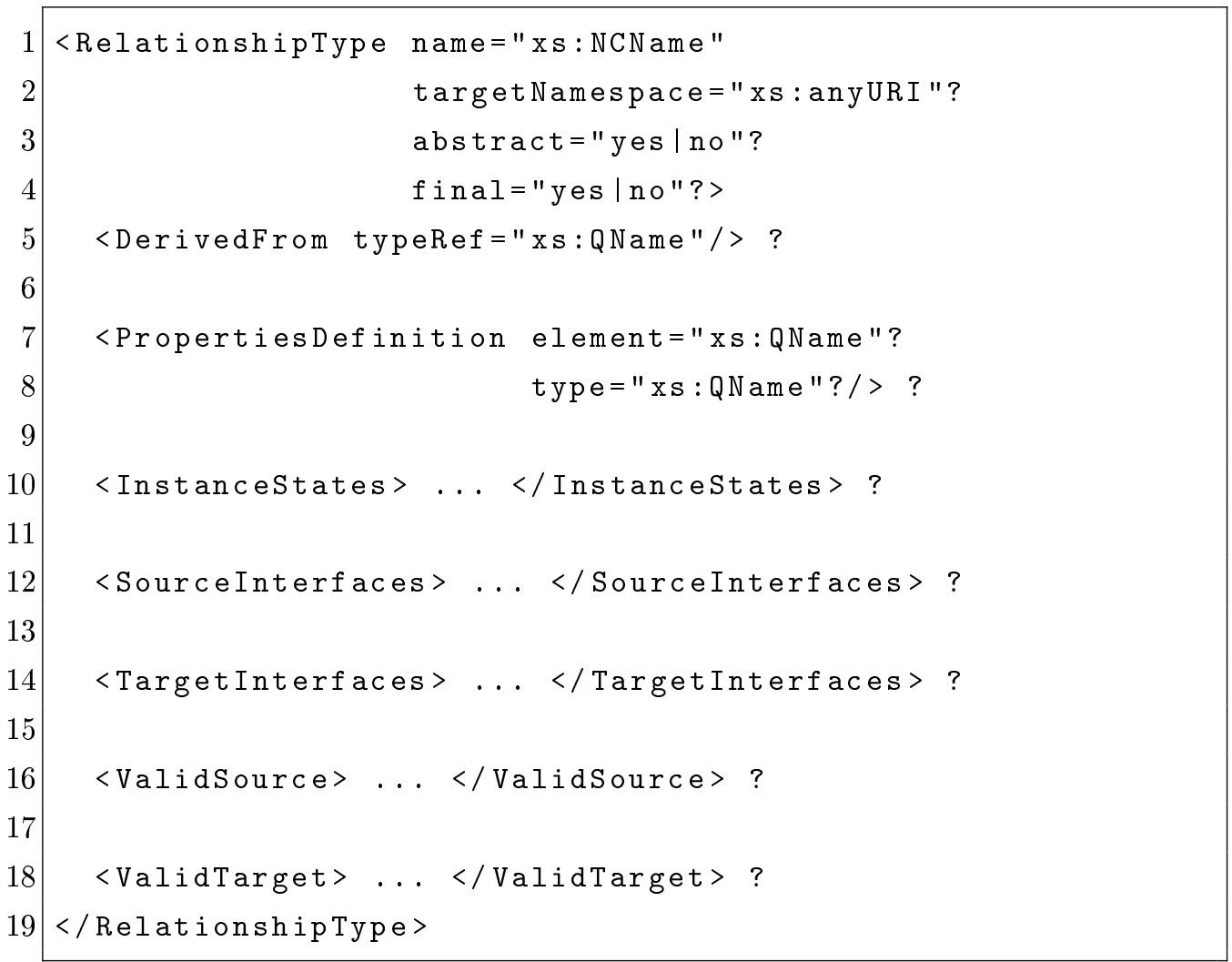

Listing 2.4: TOSCA Relationship Type element high level syntax.

Every Relationship Type requires a unique name to be identified in its own targetNamespace. If needed, the Relationship Type under definition could be specified as abstract or final. These two attributes have the same meaning of those appearing in NodeType elements. Furthermore, also the meaning (and usage) of DerivedFrom, PropertiesDefinition and InstanceStates elements is the same of those contained in NodeType elements.

The optional SourceInterfaces and TargetInterfaces elements contain definitions of manageability interfaces of a relationship of this Relation- 
ship Type (in order to actually establish the relationship between the source and the target in the deployed service). Those interface definitions are contained in nested Interface elements, whose content is the same as for NodeType interfaces.

With the optional ValidSource and ValidTarget elements, the service developer can specify the type of object that is allowed as a valid origin or target for relationships of the Relationship Type under definition.

Finally, the Relationship Type should be linked with the objects by which its interfaces are implemented. This comes with a separated Relationship TypeImplementation element. Indeed, such an element provides the collection of executables implementing the interface operations (also known as implementation artifacts $)^{3}$

\section{Requirements and capabilities}

Each NodeType element can declare to expose some requirements and capabilities. As shown in Figure 2.2, they can be expressed instantiating RequirementType and Capability Type elements (Listing 2.5) via RequirementDefinition and CapabilityDefinition elements.

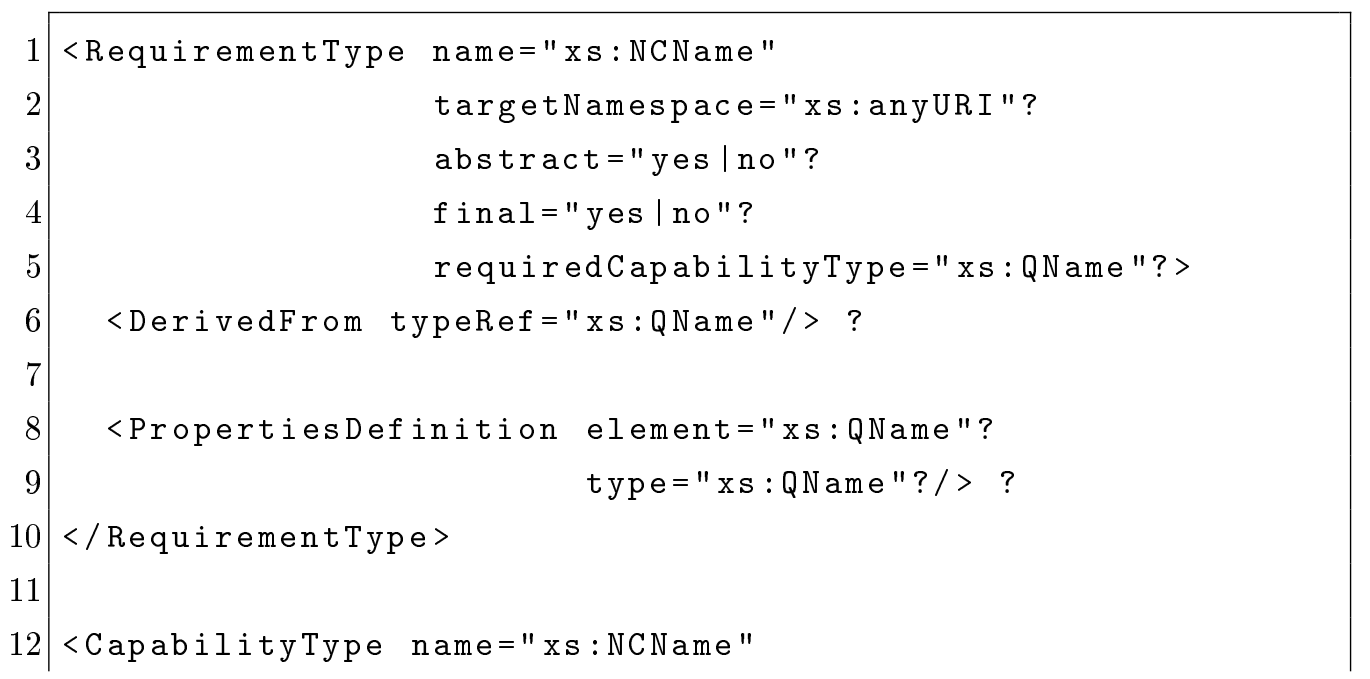

\footnotetext{
${ }^{3}$ The particular executables are defined as separate ArtifactTemplate elements and referenced from the implementation artifacts of a RelationshipTypeImplementation.
} 


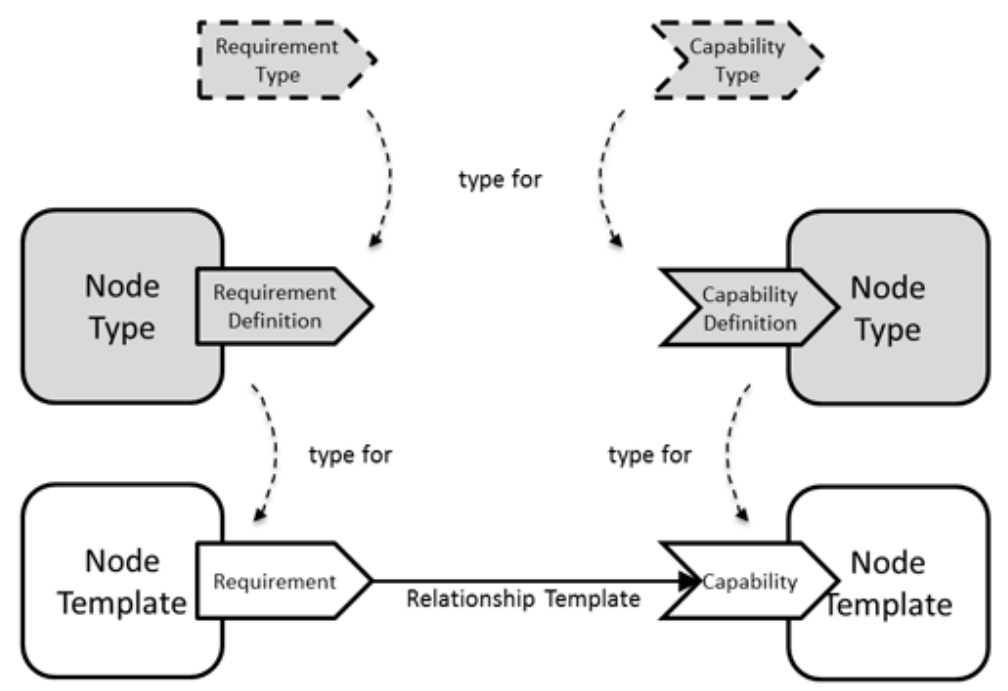

Figure 2.2: Requirement and capabilities [25]

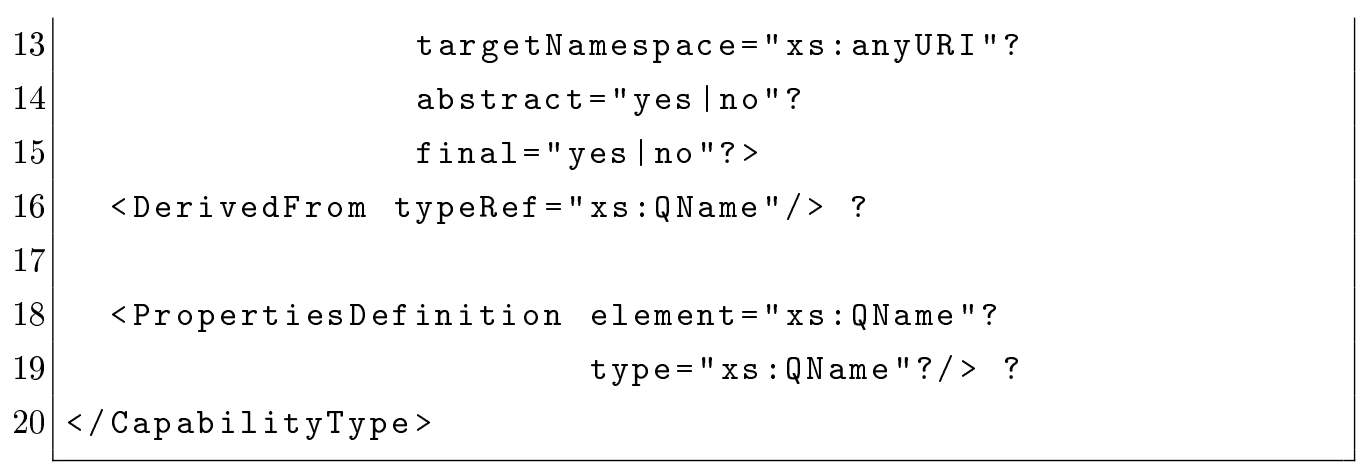

Listing 2.5: TOSCA RequirementType element and CapabilityType element high level syntax.

The meaning of all the RequirementType and CapabilityType elements common properties is the same of previous elements. The only addition is the optional requiredCapability Type attribute. It could be used to specify which CapabilityType elements ${ }^{4}$ satisfy the requirement expressed by the RequirementType element under definition.

\footnotetext{
${ }^{4}$ Since TOSCA supports inheritance, both the specified capability type and those types derived from it satisfy the considered requirement.
} 


\section{Policies}

Non-functional behaviour or QoS (Quality of Service) are defined in TOSCA by means of policies. A Policy can express such diverse things like monitoring behaviour, payment conditions, scalability, or continuous availability, for example.

As reported in Listing 2.1, a NodeTemplate can be associated with a set of Policies collectively expressing the non-functional behaviour or QoS that each instance of the NodeTemplate will expose. Each Policy specifies the actual properties of the non-functional behaviour. These properties are defined by means of a Policy Type (Listing 2.6.

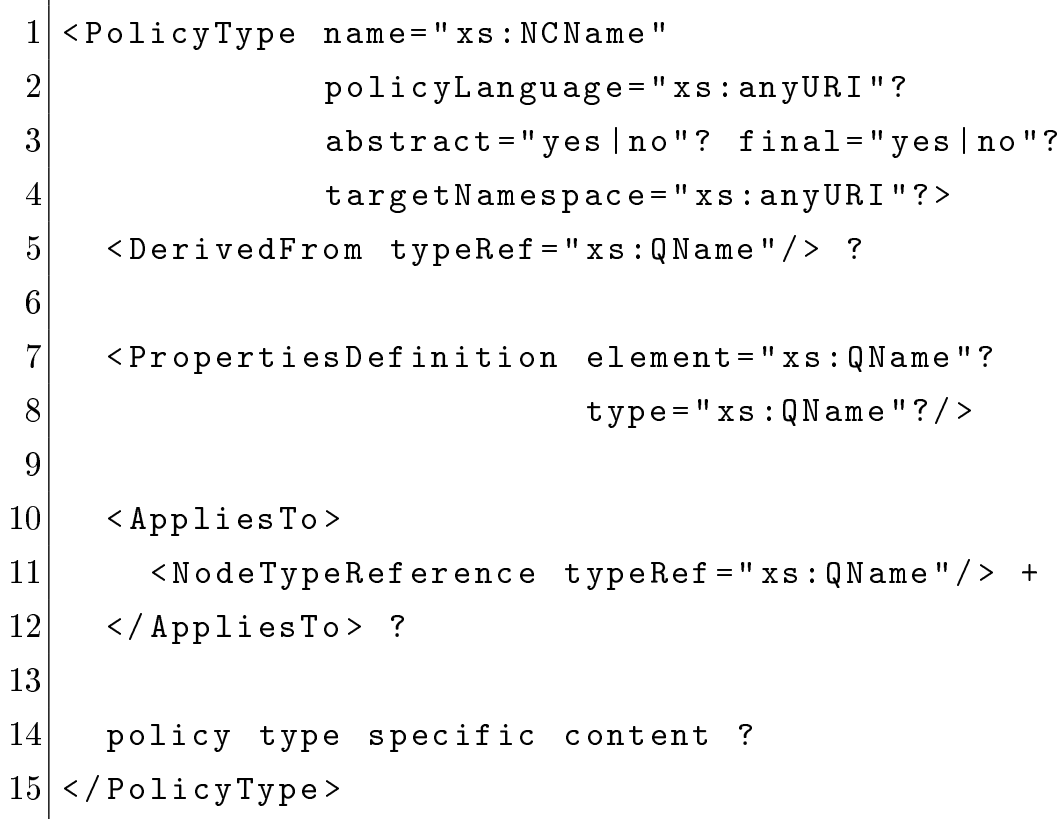

Listing 2.6: TOSCA PolicyType element high level syntax

Observe that a PolicyType could specify the non-functional behaviour it represents via both PropertiesDefinition and policy type specific content5. Furthermore, (via the AppliesTo element) a policy type can declare the set of NodeType elements it specifies non-functional behaviour for. Note

\footnotetext{
${ }^{5}$ The latter uses an arbitrary language which can be specified in the optional PolicyLanguage attribute.
} 
that being "applicable to" does not enforce implementation (e.g., in case a Policy Type expressing high availability is associated with a WebserverNodeType, an instance of the WebserverNodeType is not necessarily high available). Whether or not an instance of a NodeType to which a PolicyType is applicable will show the specified non-functional behaviour, is determined by a NodeTemplate of the corresponding NodeType.

Once the general properties have been defined in a PolicyType, their actual values are provided by one or more PolicyTemplate elements (Listing 2.7).

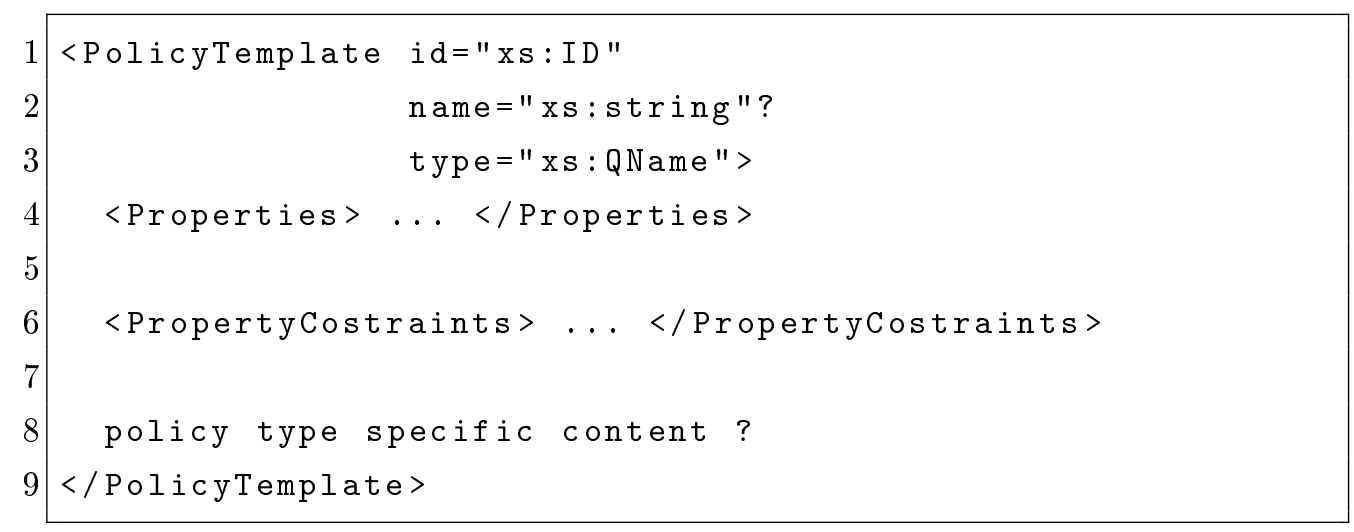

Listing 2.7: TOSCA PolicyTemplate element high level syntax.

A PolicyTemplate defines the invariant properties of the non-functional behaviour to be represented. Its variant properties are setted in a Policy element of a NodeTemplate. This is because those properties result from the actual usage of a PolicyTemplate in the NodeTemplate under consideration (e.g., a Policy Template for italian customers yearly payments will set the paymentPeriod property to "yearly" and the currency property to "EUR", leaving the amount property open; the amount property will be set when the corresponding Policy Template is used for a Policy within a NodeTemplate). 


\subsection{A TOSCA example}

Suppose that a cloud application developer wants to build the weather forecast cloud application in Figure 2.3. Her work starts with the creation

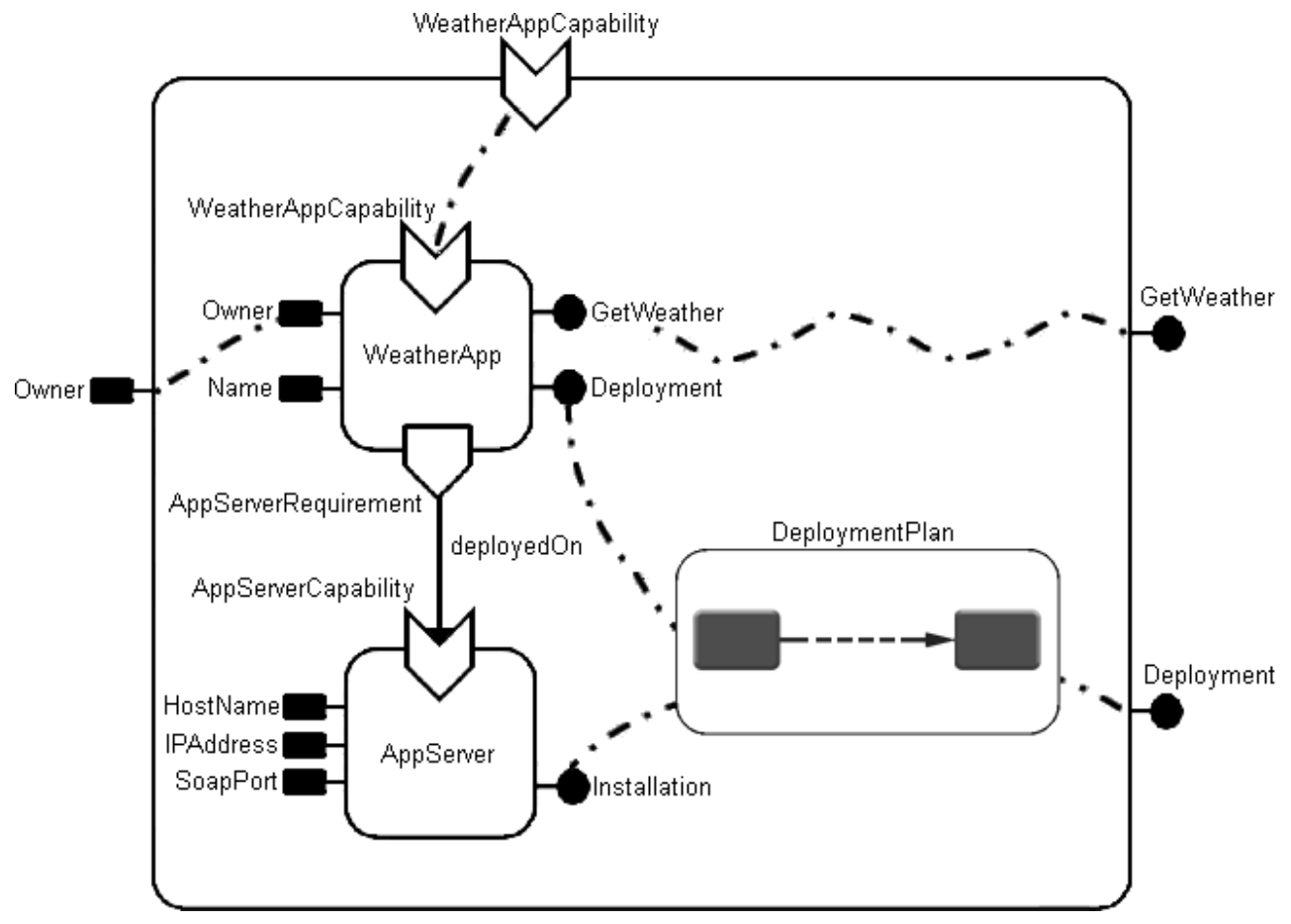

Figure 2.3: TOSCA cloud application example.

of a new Definitions document (Listing 2.8) in which to put all required elements. To enhance readability we are going to show each needed definition in a separate section.

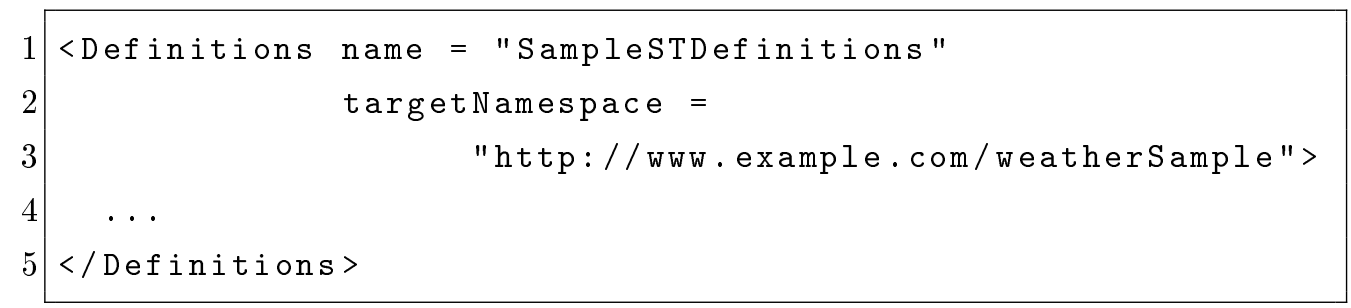

Listing 2.8: TOSCA example Definitions document. 


\section{Requirement and capability types}

Before writing the NodeType and RelationshipType elements needed by the desired application, the developer must ensure she has all the necessary requirement and capability types. In other words, she has to write the AppServerRequirement, AppServerCapability and WeatherAppCapability definitions (Listing 2.9 and to include them in the Definitions element.

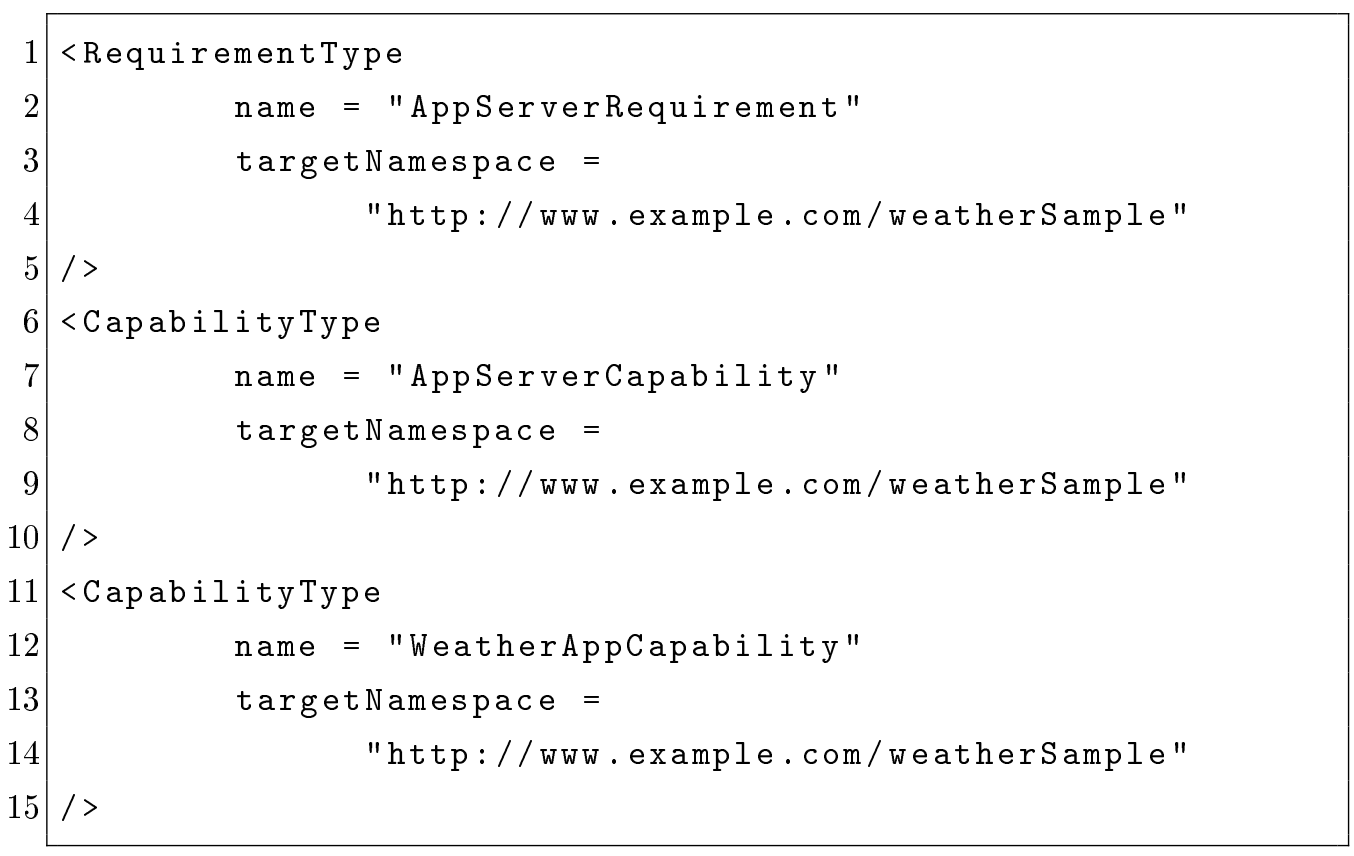

Listing 2.9: TOSCA example requirement and capability types definitions.

Please note that the exposed requirement and capabilities definitions are only composed by their name. This is because they will be used only in establishing the meaning of desired relationships.

\section{Node types}

Once requirement and capability types are defined, the application developer needs only one other ingredient to proceed in NodeType definitions: 
the properties structures definition (Listing 2.10).

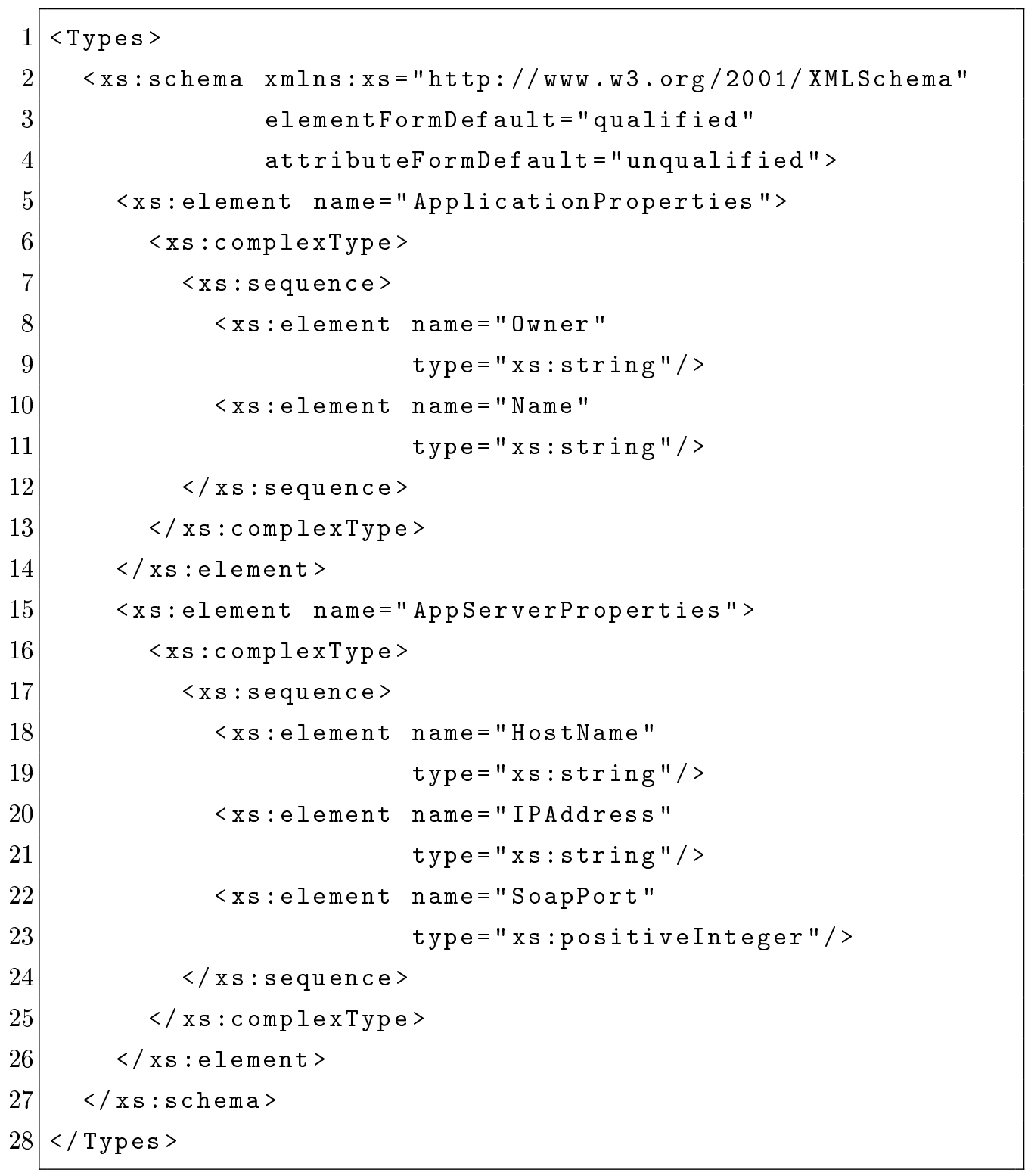

Listing 2.10: Properties structure definitions required in our TOSCA example.

As the Types element is included in the Definitions document, she can proceed defining the required NodeType elements (Listing 2.11).

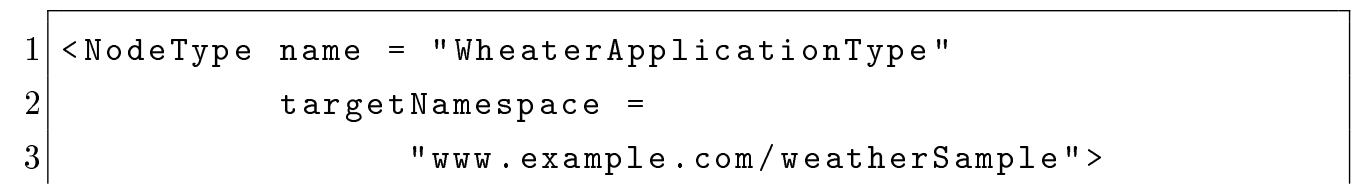




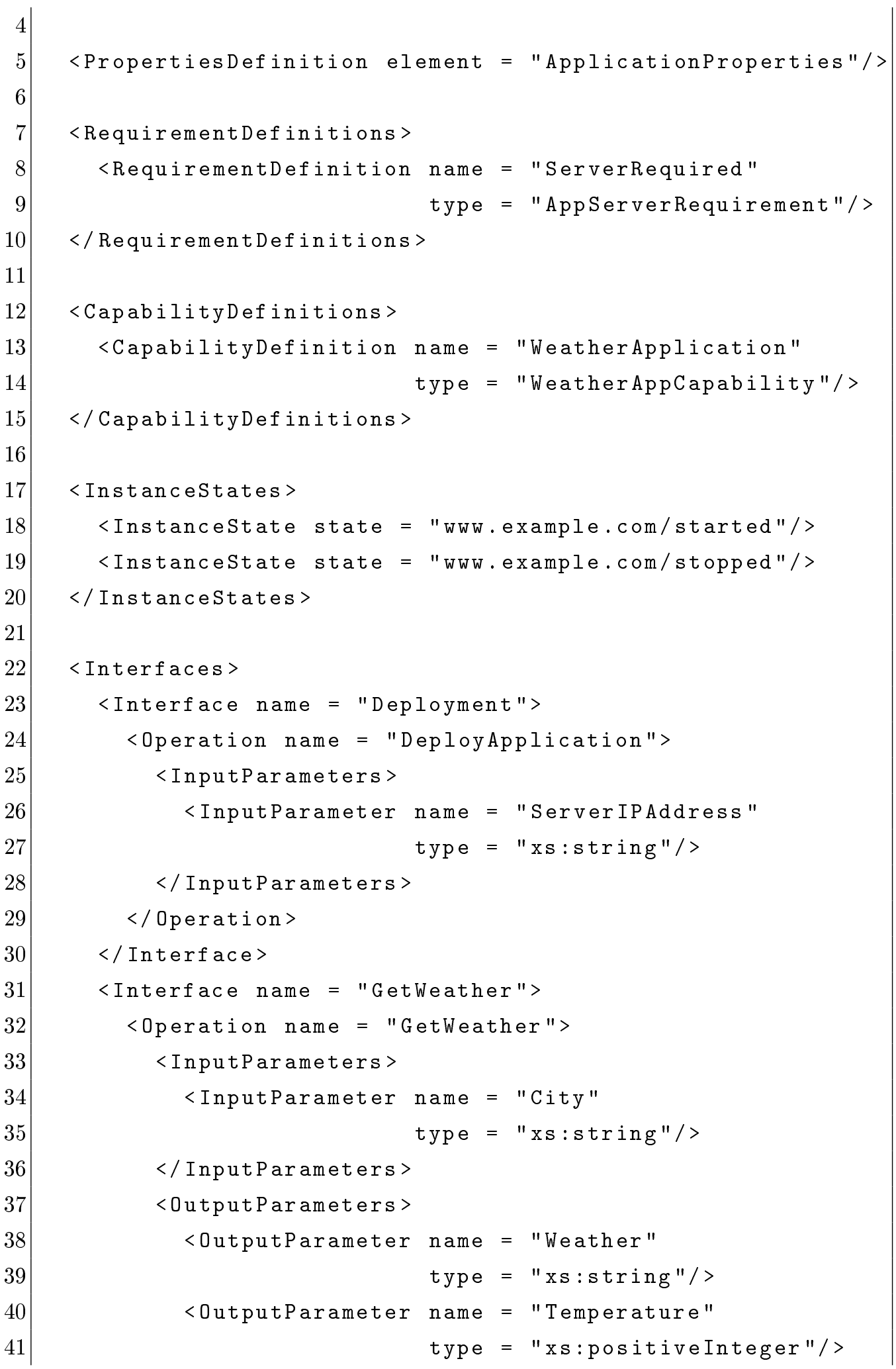




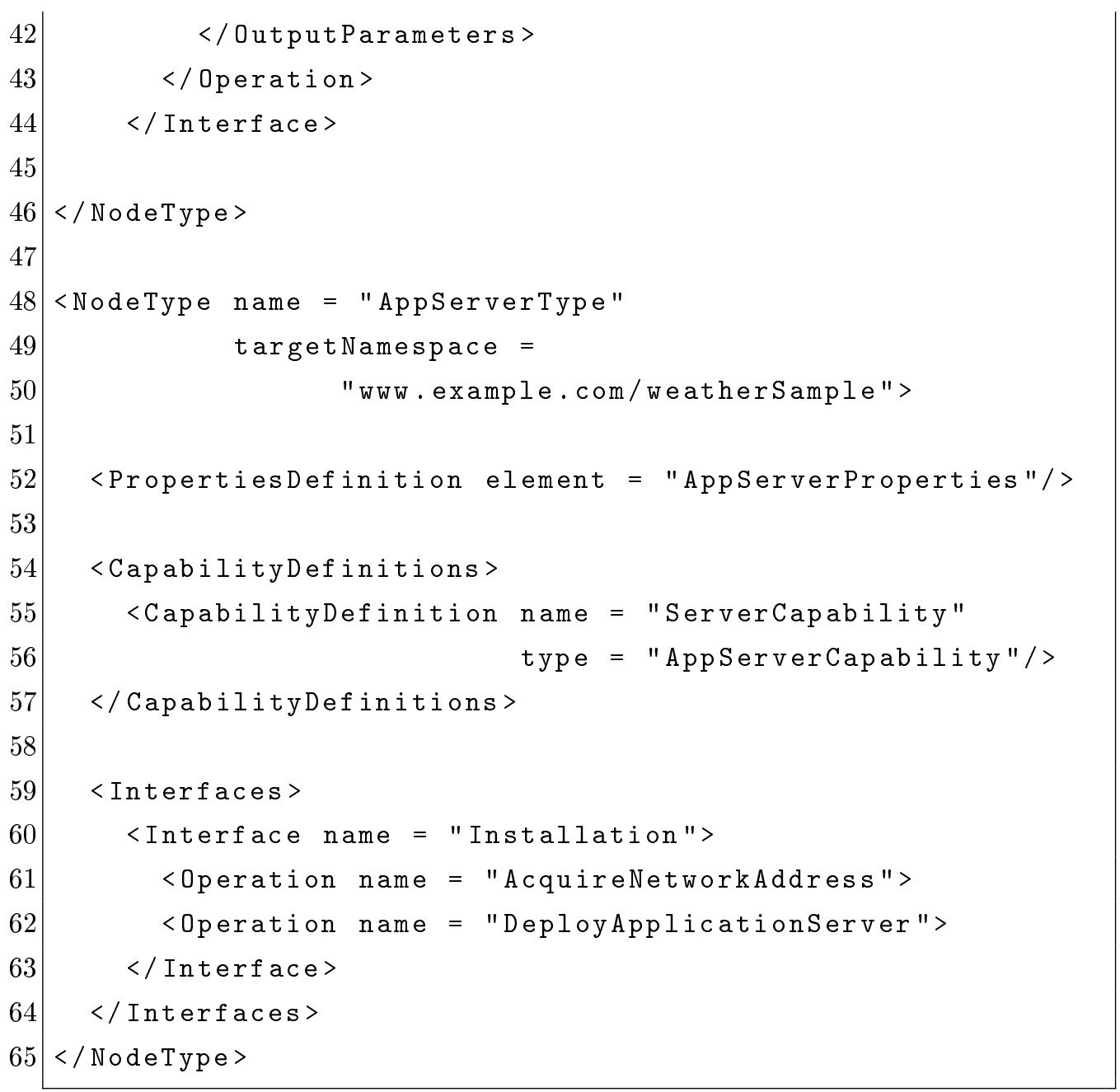

Listing 2.11: TOSCA example NodeType definitions.

\section{Relationship types}

Looking at Figure 2.3, we observe that the desired service topology contains a relationship between the WeatherApp's requirement and the AppServer's capability. So, the desired relationship's type must be defined by the application developer (Listing 2.12).

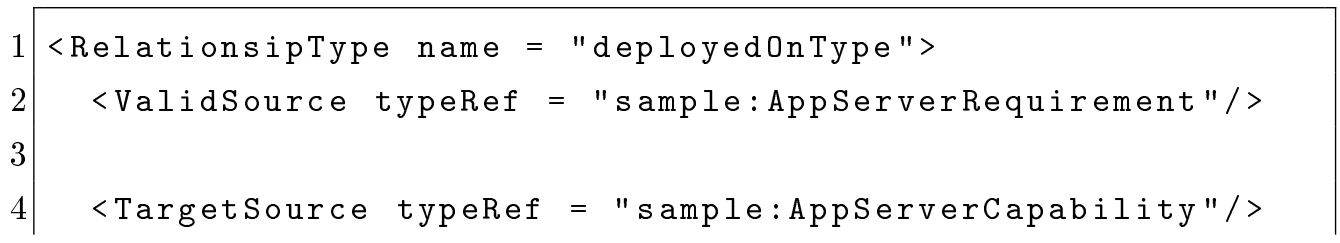


$5 \mid</$ Relationshiptype >

Listing 2.12: TOSCA example Relationship Type definition.

\section{Topology template}

In the previous sections we have shown the type definitions made by the weather forecast application developer. Now, she can proceed in instantiating those types in order to build up the desired service topological aspects (Listing 2.13).

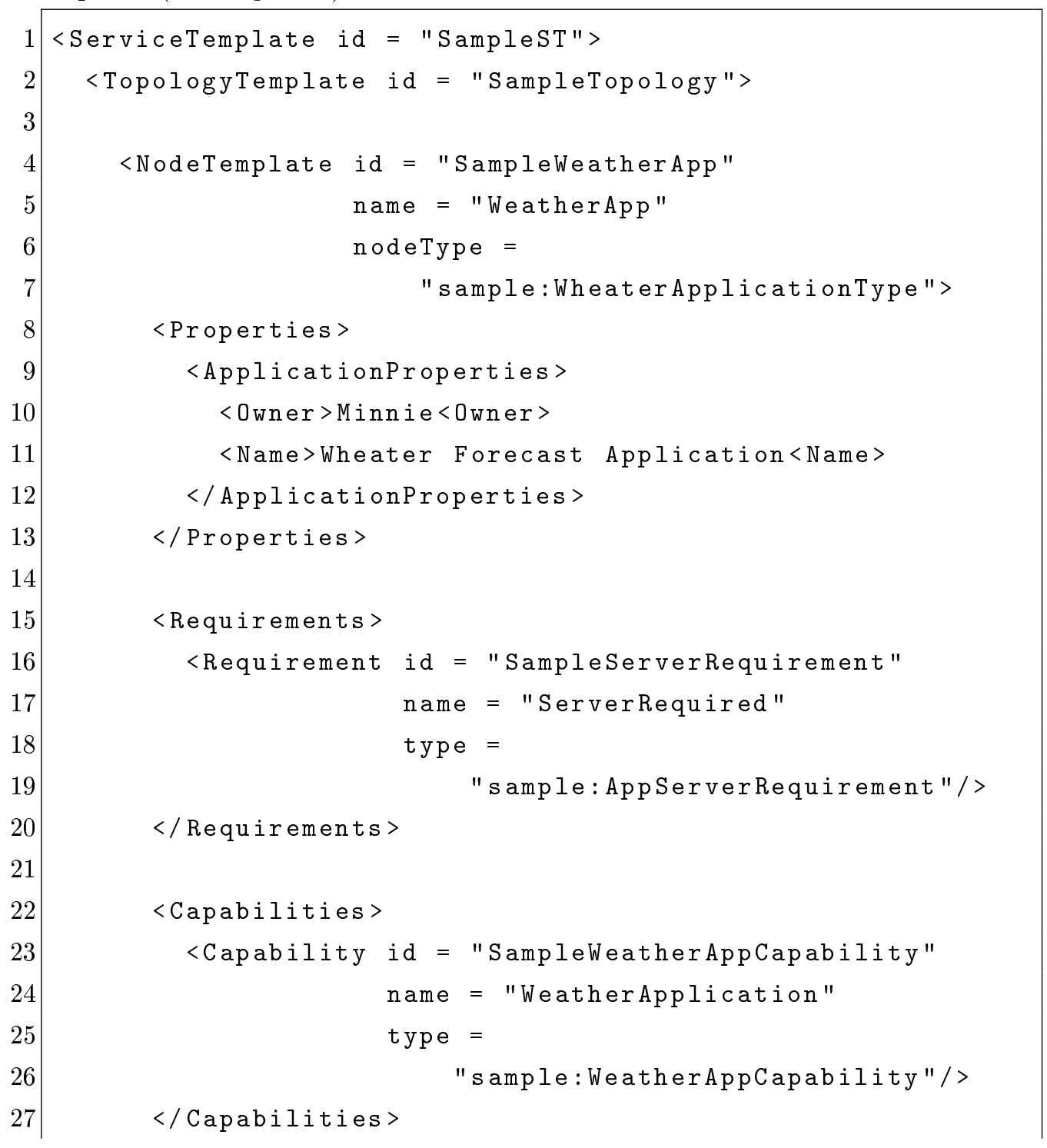




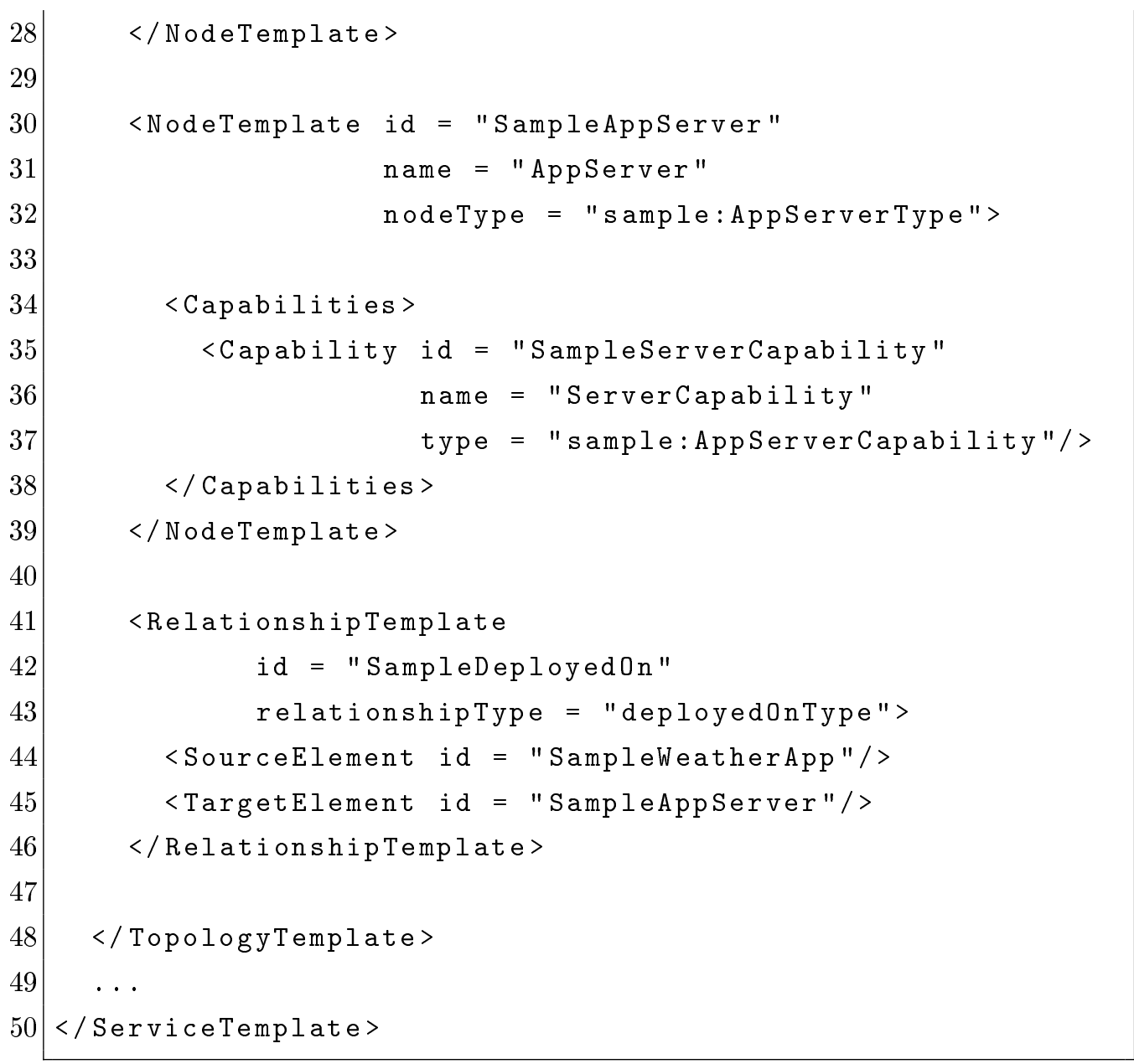

Listing 2.13: TOSCA example TopologyTemplate definition.

\section{Plans}

Once the service topology has been defined, the application developer must define all desired management aspects. A possible solution to the exposed issue is to use URI references to already existing plans (Listing 2.14 ).

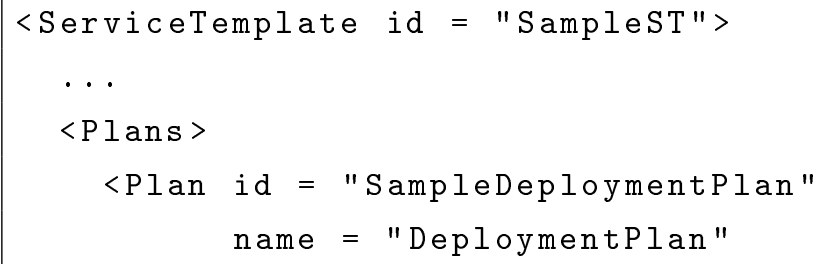




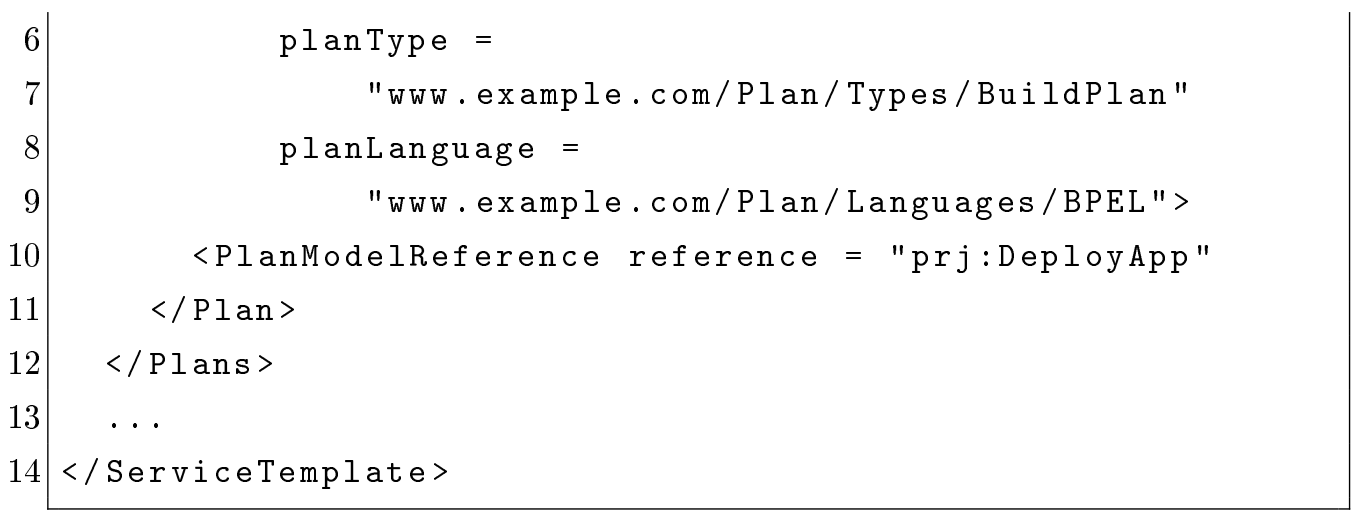

Listing 2.14: TOSCA example Plans definition.

\section{Service template and boundaries}

Last but not least, the application developer must ensure that her service only exposes what she wants. This is done via the BoundaryDefinitions element (Listing 2.15).

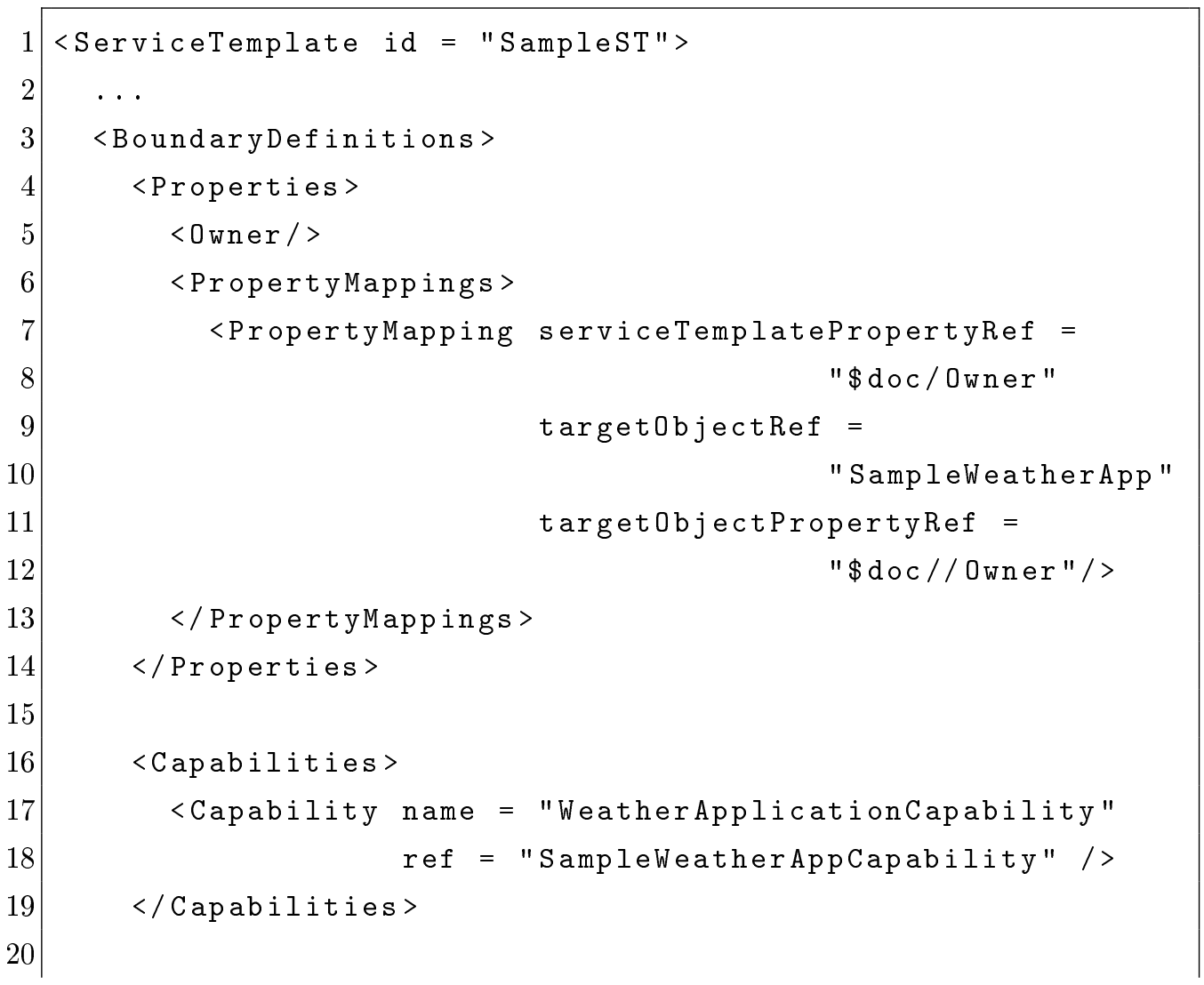




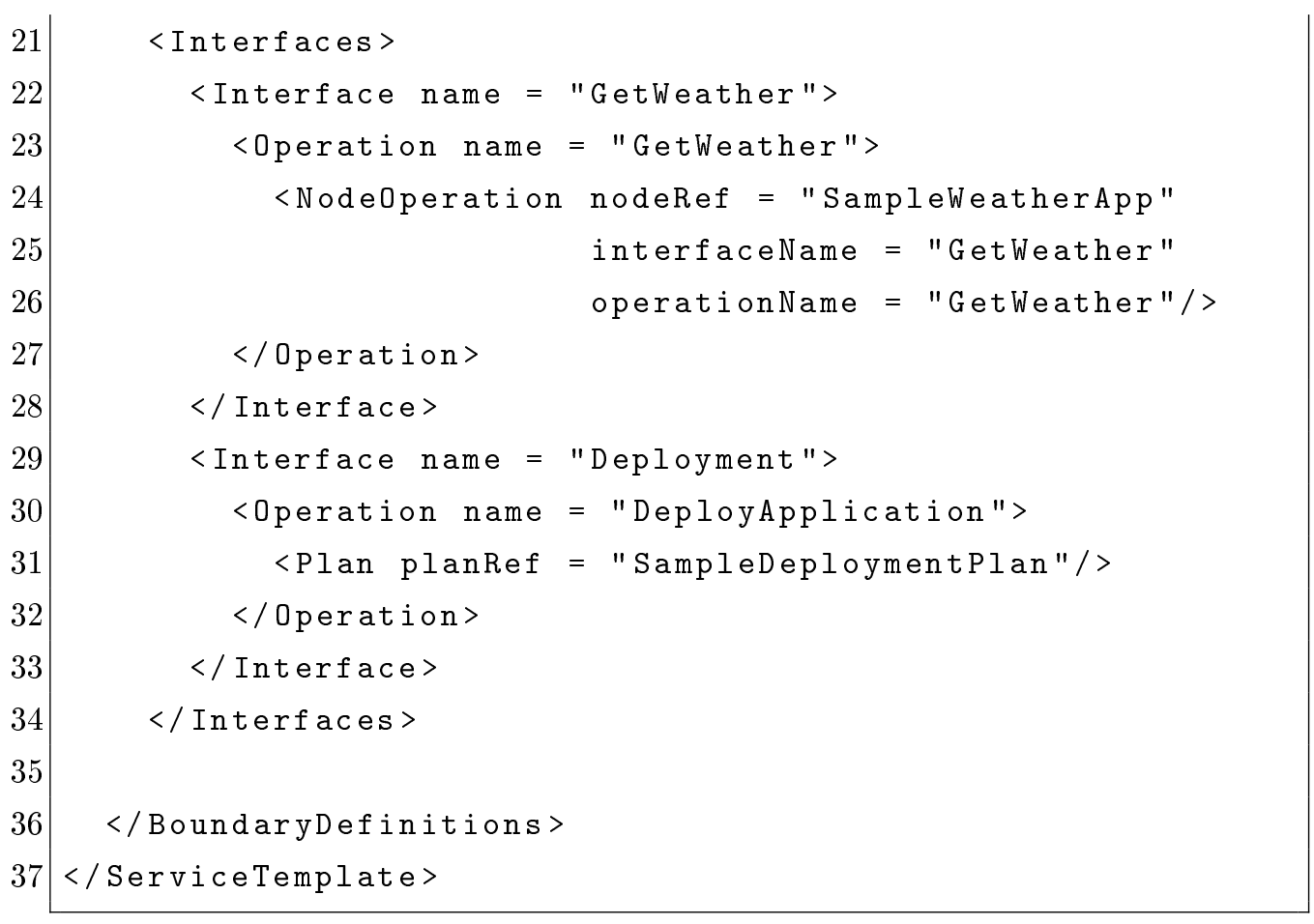

Listing 2.15: TOSCA example boundary definitions. 


\section{Chapter 3}

\section{Node Type black-box matching (and adaptation)}

The objective of this chapter is to give a notion of black-box matching between NodeType and ServiceTemplate elements. Once this notion is available, it can be used as a first step in understanding whether a TOSCA service component could be replaced with an available cloud service.

To provide such a compatibility notion we start by defining an exact matching between the two TOSCA elements under consideration (Section 3.1). Then, observing that there is no need to exactly match the elements, we define the notion of plug-in matching (Section 3.2). Finally, using ontologies to ignore non-relevant syntactic differences, we derive the flexible matching notion (Section 3.3).

We also describe how a Service Template that plug-in or flexibly matches the desired NodeType can be suitably adapted so as to exactly match it. 


\subsection{Exact matching}

We consider the problem of matching a NodeType $N$ with a ServiceTemplate ST. The TOSCA Primer [26] gives an informal notion of matching between these two elements. Referring to Figure 3.1, the Primer authors

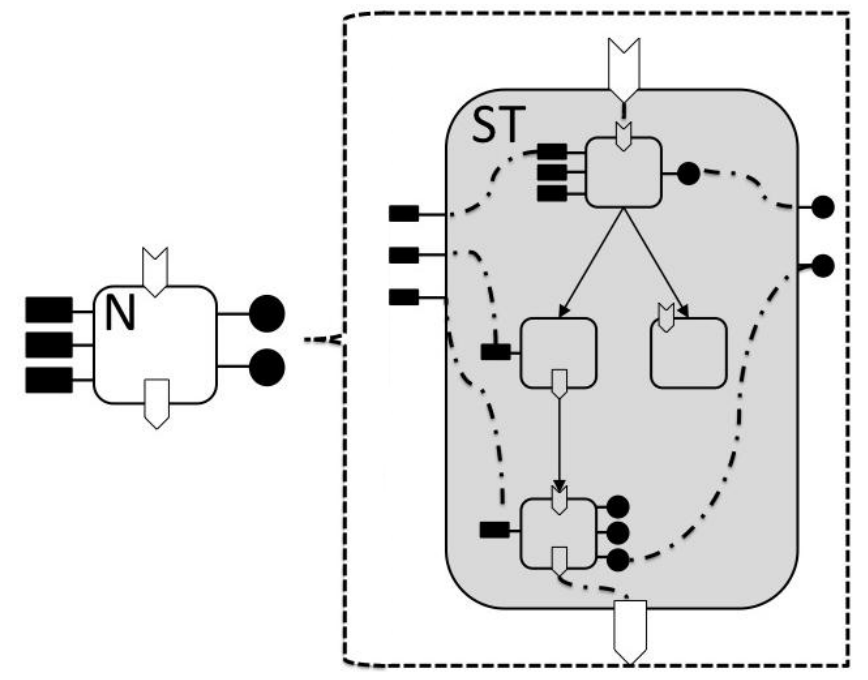

Figure 3.1: ServiceTemplate substituting a NodeType [26].

say:

«Service template ST may substitute node type $N$ because the boundary of ST matches all defining elements of $N$ : all properties, operations, requirements and capabilities of ST match exactly those of $N . »$

No additional matching information is given. So, we want to formalize this notion.

\subsubsection{Definition of exact matching}

What we want to do is to define an operator "三" which takes a pair $\langle$ NodeType, ServiceTemplate $\rangle$ and returns a truth value (which represents 
whether the two elements exactly match or not).

Definition 3.1. A NodeType $N$ exactly matches a ServiceTemplate $S T$ $(N \equiv S T)$ if and only if:

$$
\begin{gathered}
\text { N.RequirementDefinitions } \equiv_{R} \text { ST.Requirements } \wedge \\
\text { N.CapabilityDefinitions } \equiv_{C} \text { ST.Capabilities } \wedge \\
\text { PolicyType applicable to } N \equiv_{P O} \text { ST.Policies } \wedge \\
\text { N.PropertiesDefinition } \equiv_{P R} \text { ST.Properties } \wedge \\
\text { N.Interfaces } \equiv_{I} \text { ST.Interfaces }
\end{gathered}
$$

To understand what Definition 3.1 means, we need to analyse each of the given conditions.

\section{Exact matching of requirements}

Looking at the syntax of the elements interested in this kind of matching (Listing 3.1), we can define the required condition.

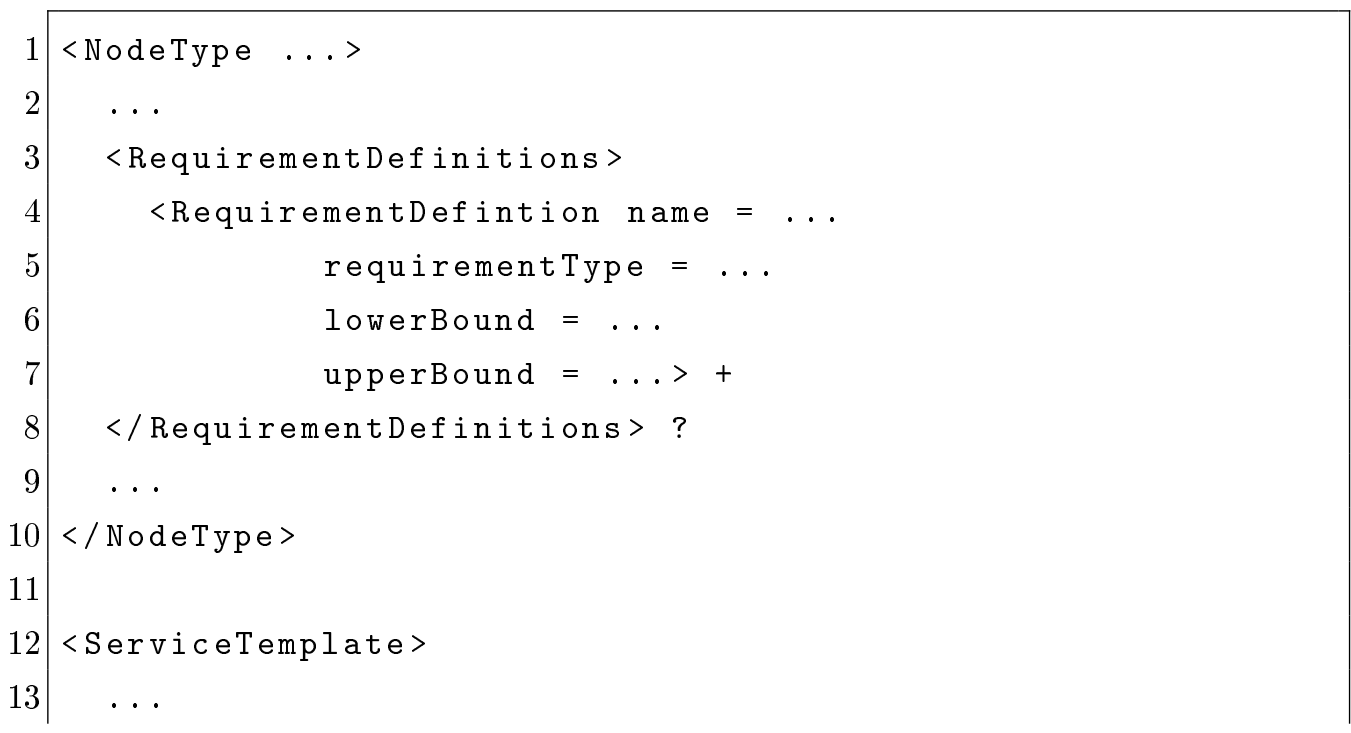




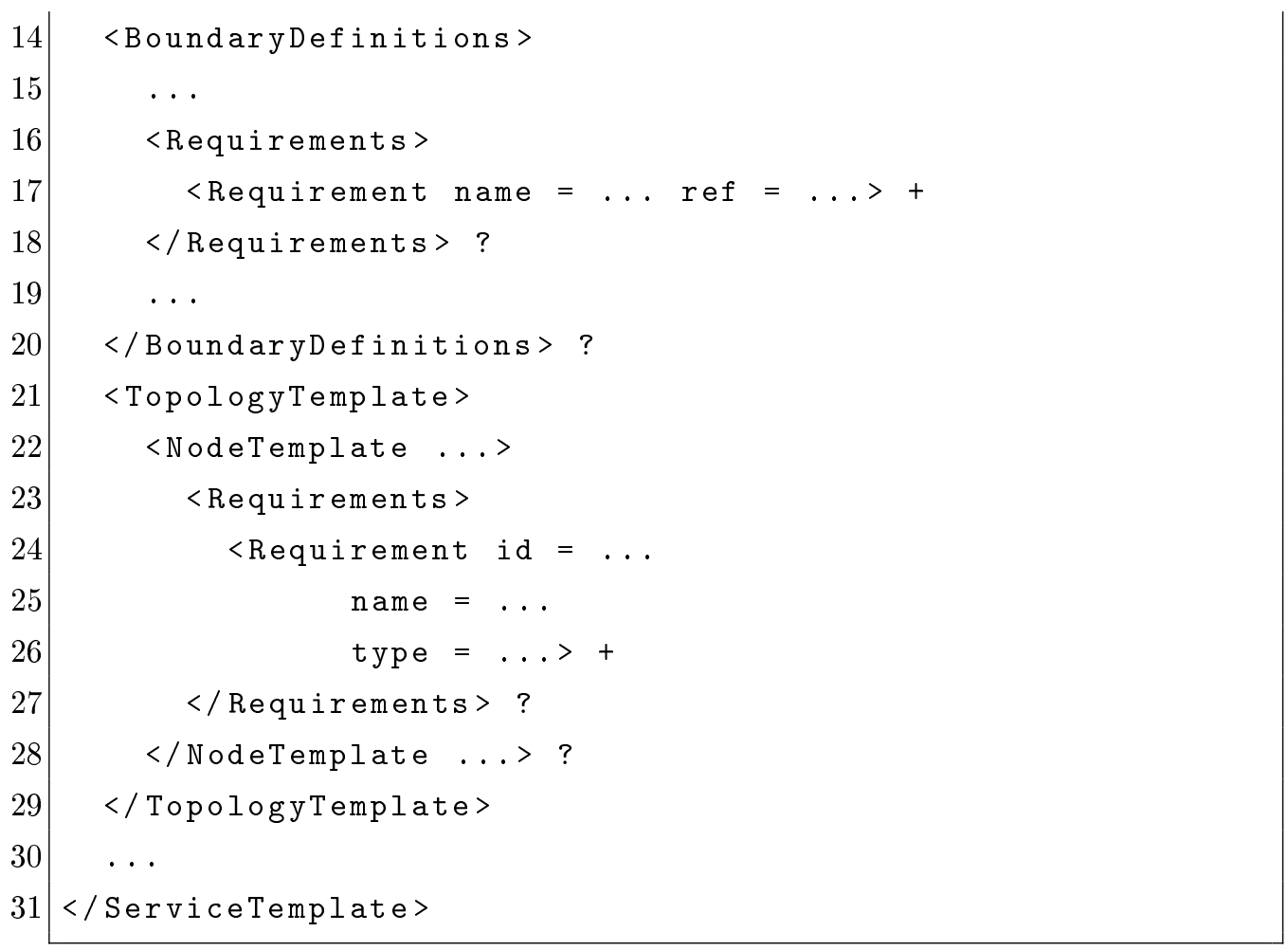

Listing 3.1: High level syntax of TOSCA NodeType and ServiceTemplate elements interested in requirements matching.

The Topology and Orchestration Specification for Cloud Applications [25] says that:

«The name and type of the Requirement MUST match the name and type of a RequirementDefinition in the NodeType specified in the type attribute of the NodeTemplate.»

Extending this concept to our problem, we can state that each RequirementDefinition in the NodeType must have the same name and type of exactly one of the ServiceTemplate's Requirements (referred by the ref attribute in the BoundaryDefinitions element).

Furthermore, looking at NodeType specification [25], the authors says that the NodeType under consideration exposes also the requirements (if not overridden) of the NodeType it is derived from. This means that its 
Chapter 3. NodeType black-box matching (and adaptation)

requirements consist of the (set theoretic) union of the requirements it defines and the requirements it inherits from the parent NodeType.

The above reasoning let us define the condition needed in Definition 3.1 .

Definition 3.2. Let $N$ be a NodeType and $S T$ be a ServiceTemplate. We say that

$$
\text { N.RequirementDefinitions } \equiv_{R} \text { ST.Requirements }
$$

if and only if

1. $\forall$ RequirementDefinition $x \in N$.RequirementDefinitions

$\exists$ ! Requirement $y \in S T$.Requirements:

$x . n a m e=y . n a m e \wedge x . r e q u i r e m e n t T y p e=y . r e f . t y p e$

2. $\forall$ Requirement $y \in S T$.Requirements

$\exists$ ! RequirementDefinition $x \in N$.RequirementDefinitions:

$x . n a m e=y . n a m e \wedge x$. requirementType $=$ y.ref.type

\section{Exact matching of capabilities}

The high similarity between the capabilities syntax and the requirements one leads to the following definition.

Definition 3.3. Let $N$ be a NodeType and $S T$ be a ServiceTemplate. We say that

\section{N.CapabilityDefinitions $\equiv_{C}$ ST.Capabilities}

\footnotetext{
${ }^{1}$ Using both the conditions we ensure the one-to-one correspondence between the requirements of the two elements.
} 
if and only if:

1. $\forall$ CapabilityDefinition $x \in$ N.CapabilityDefinitions

$\exists$ ! Capability $y \in S T$. Capabilities:

$x$. name $=y$. name $\wedge$ x.capability Type $=$ y.ref.type

2. $\forall$ Capability $y \in S T$.Capabilities

$\exists$ ! CapabilityDefinition $x \in N$. CapabilityDefinitions:

$x$. name $=y$. name $\wedge$ x.capability Type $=$ y.ref.type

\section{Policies compatibility}

As before, we need to look at the syntax of interested elements to define the policies matching condition.

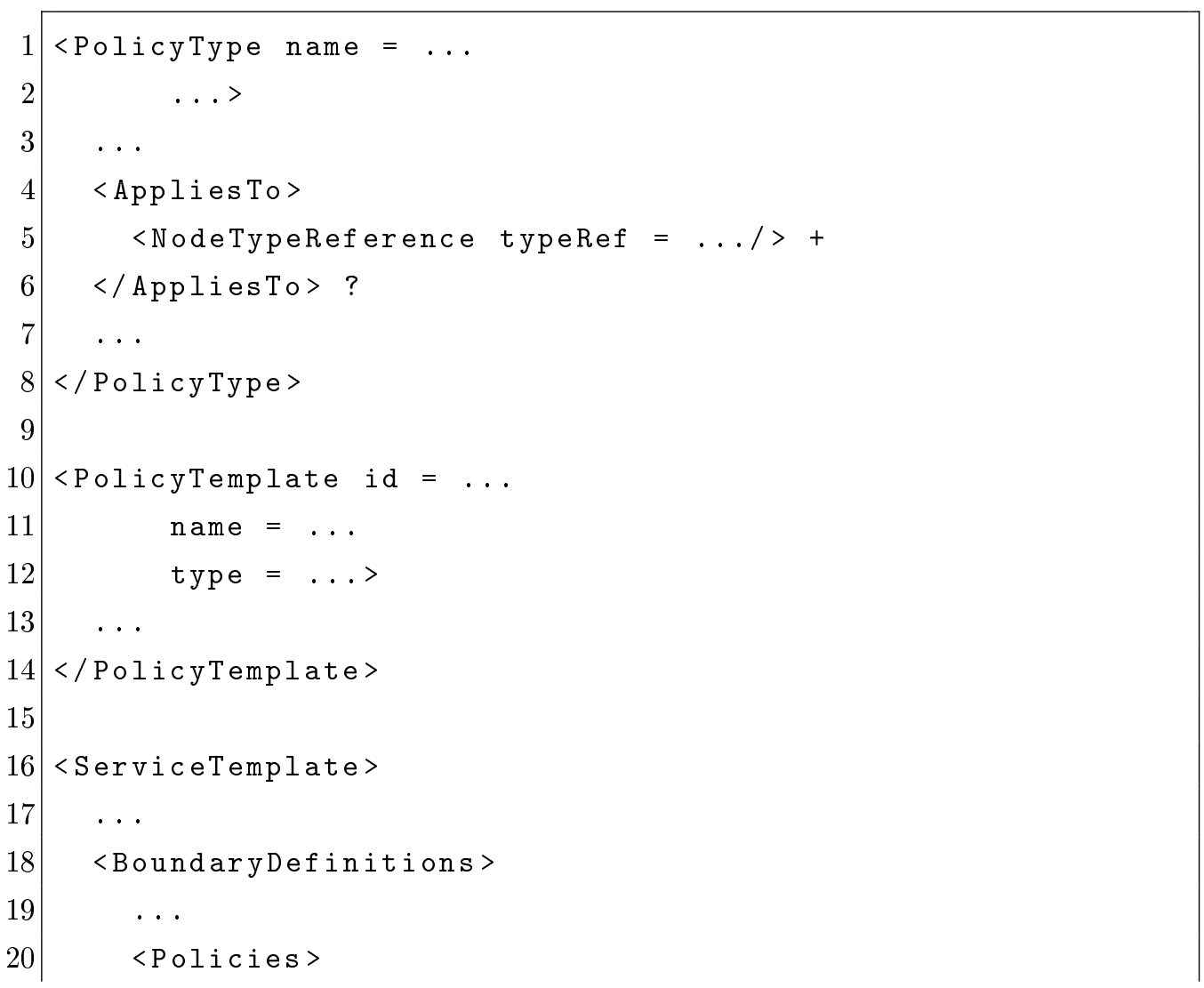




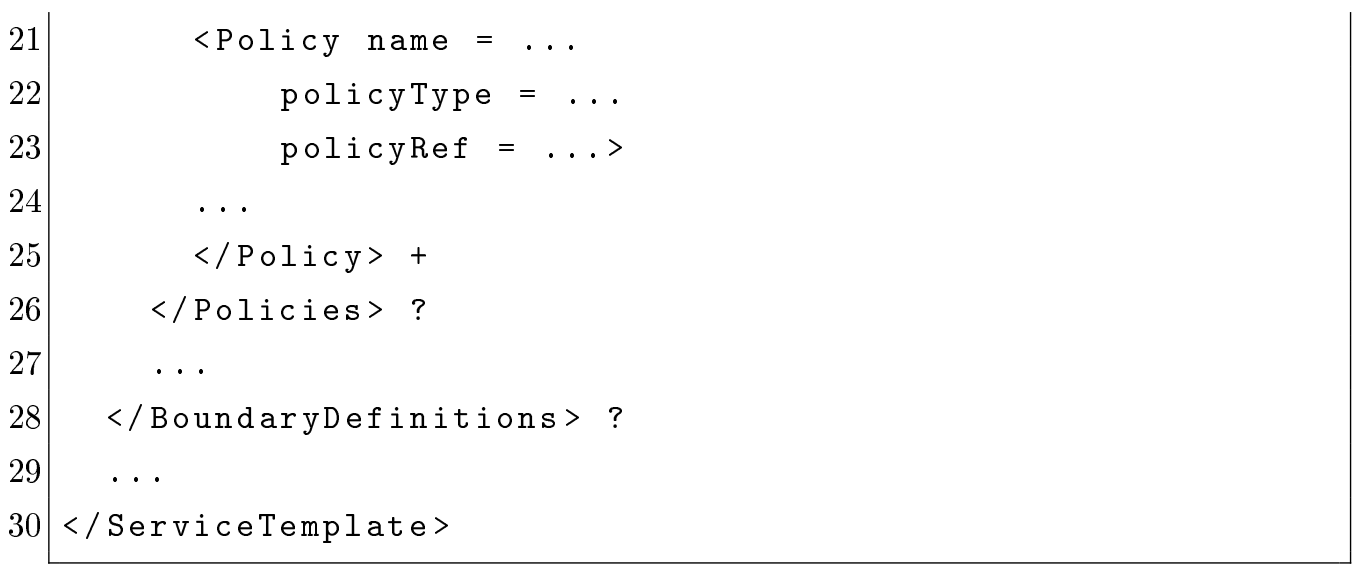

Listing 3.2: High level syntax of TOSCA elements interested in policies matching.

Looking at Listing 3.2 , we immediately observe the lack of NodeType element. In this case we have to consider PolicyType, PolicyTemplate and ServiceTemplate elements. This is because a NodeType cannot expose any kind of policy, but a policy can specify which set of NodeType elements it is applicable to.

Assumption 3.1. If a PolicyType $P_{T Y}$ does not contain any AppliesTo element, then $P_{T Y}$ will be applicable to any NodeType.

The above consideration suggests that if we want to substitute a NodeType $N$ with a ServiceTemplate $S T$, then the latter must expose policies applicable to the former. So, let us define the infix operator $\succ$ to indicate that the PolicyType of the considered policy (on the left of $\succ$ ) is applicable to the NodeType (on the right of $\succ$ ).

Definition 3.4. The infix operator

$$
\succ:(\{\text { PolicyType }\} \cup\{\text { PolicyTemplate }\}) \times\{\text { NodeType }\} \rightarrow\{\text { true }, \text { false }\}
$$

is defined as follows:

- let $P_{T Y}$ be a PolicyType and $N$ a NodeType. $P_{T Y} \succ N$ is true 
if and only if the $P_{T Y}$.AppliesTo element (is empty or) contains a NodeTypeReference to $N$ or to a NodeType from which $N$ is derived;

- let $P_{T M P}$ be a PolicyTemplate and $N$ a NodeType. $P_{T M P} \succ N$ is true if and only if $P_{T M P}$.type $\succ N$ is true.

Now we can define the desired condition.

Definition 3.5. Let $N$ be a NodeType and ST a ServiceTemplate. The set of PolicyTypes applicable to $N$ exactly matches $\left(\equiv_{P O}\right)$ ST.Policies if and only if:

$\forall S T$.Policies.Policy x, (x.policyType $\succ N \vee x$.policyRef $\succ N)$

\section{Exact matching of properties}

Understanding how to match NodeType.PropertiesDefinition and Service Template properties requires to look at their syntax (Listing 3.3).

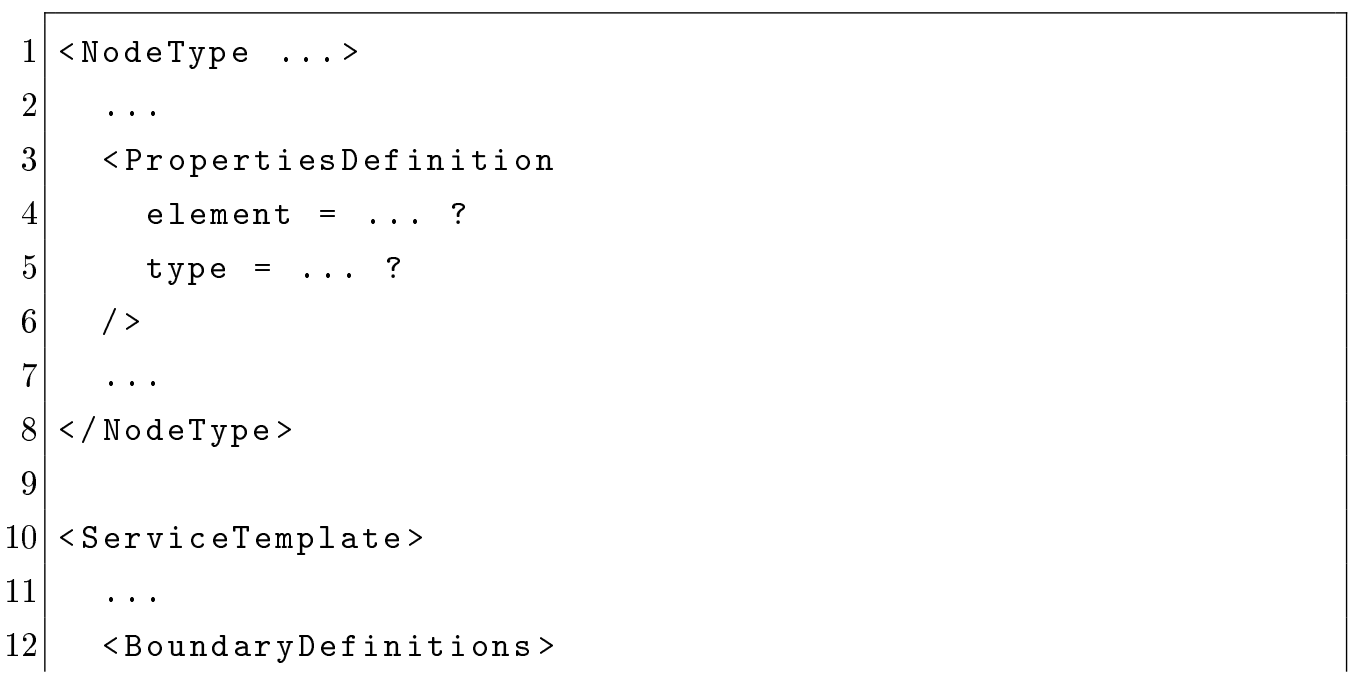




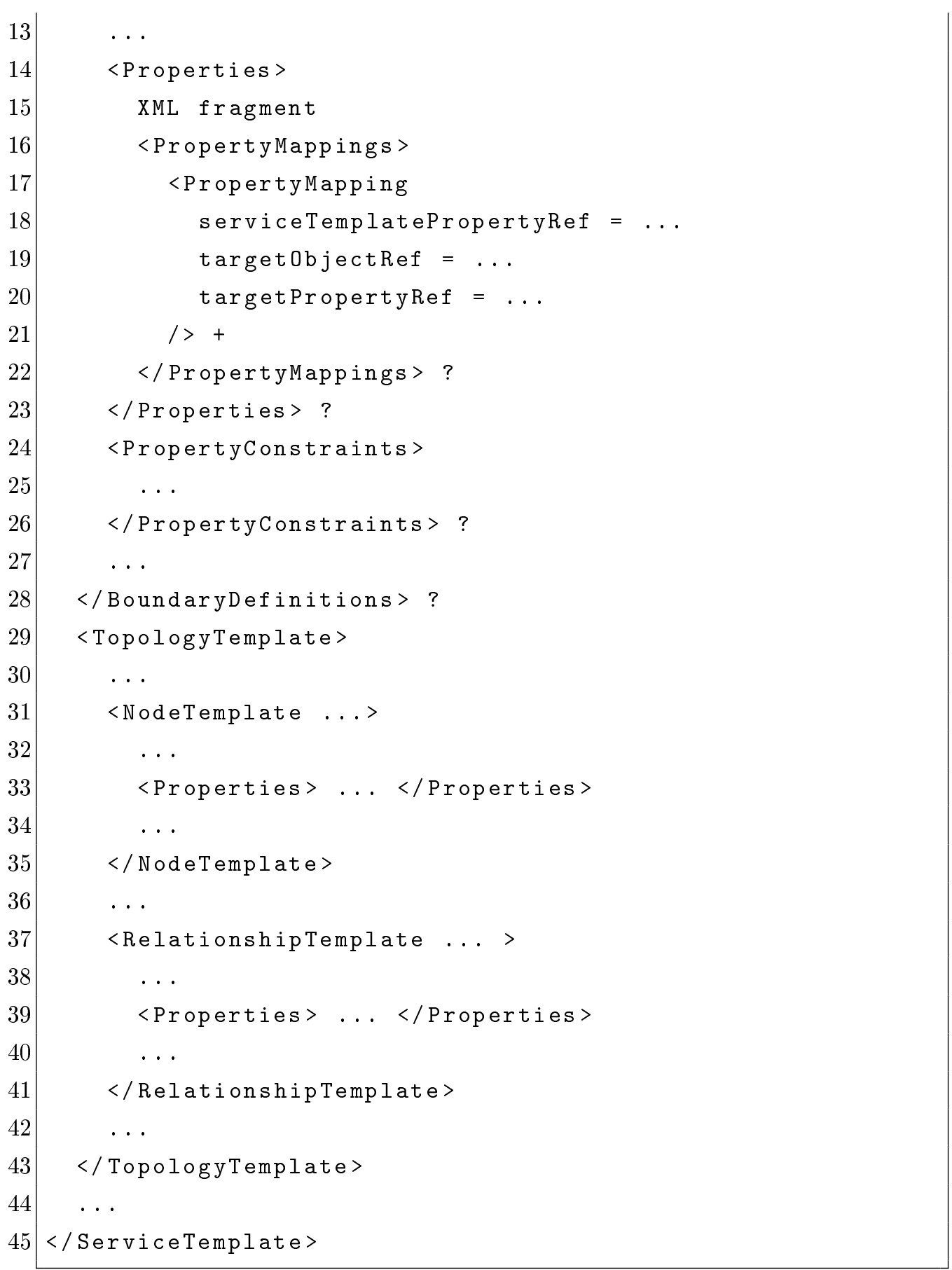

Listing 3.3: High level syntax of TOSCA NodeType and ServiceTemplate elements interested in properties matching. 
Chapter 3. NodeType black-box matching (and adaptation)

Observe that there is a different properties definition between NodeType and ServiceTemplate elements:

- the former specifies (via one of the element and type attributes) the XML schema of the NodeType observable properties;

- the latter specifies ServiceTemplate actual property values (and constraints) using a XML fragment and mapping the exposed properties to the ones of nested elements (using the serviceTemplatePropertyRef attribute - to refer the property defined in the XML fragment - and targetObjectRef and targetObjectPropertyRef attributes - to identify the property of the nested element).

This means that, because of lack of values (and constraints) in NodeType elements, we can only match property types.

Furthermore, in the TOSCA specification [25], the authors assume that the XML type representing the NodeType properties extends the XML type of the properties of the NodeType referenced in the DerivedFrom element. This implies that we do not have to worry about what the NodeType element inherits from its parent.

The above considerations let us define the desired condition:

Definition 3.6. Let $N$ be a NodeType and $S T$ a ServiceTemplate. We say that

$$
\text { N.PropertiesDefinition } \equiv_{P R} \text { ST.Properties }
$$

if and only if the XML type of ST.Properties is the same as the one defined with N.PropertiesDefinition 


\section{Exact matching of interfaces}

Finally, we want to analyze Interfaces matching. So, as done before, we need to take a look to the elements interested in this matching (Listing 3.4).

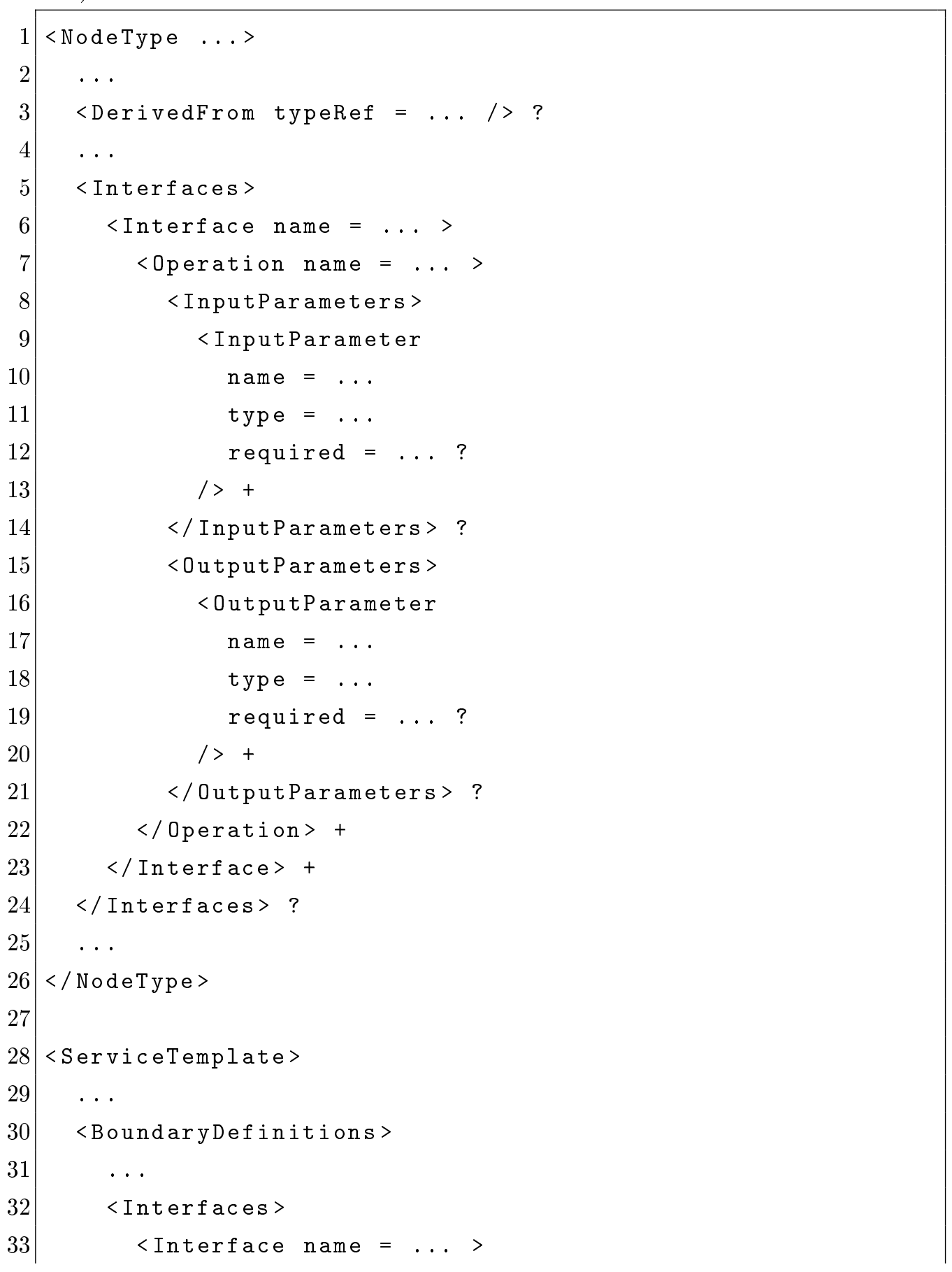


Chapter 3. NodeType black-box matching (and adaptation)

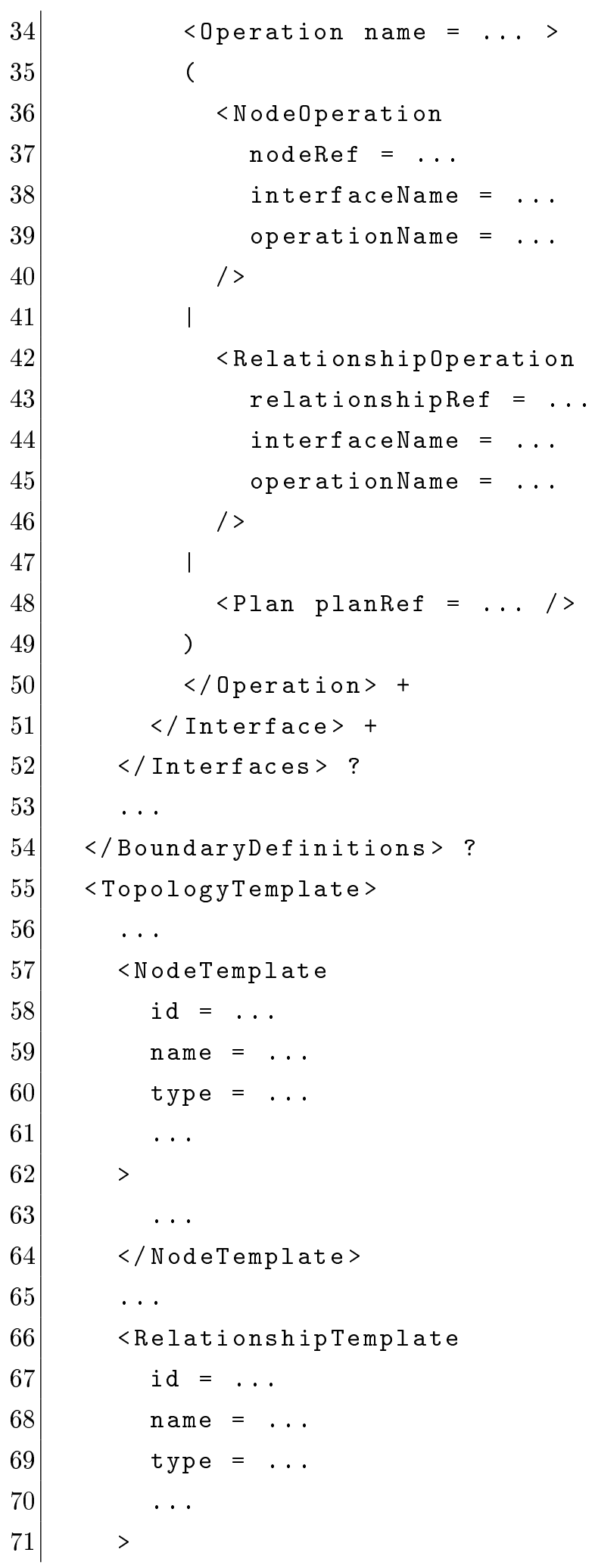




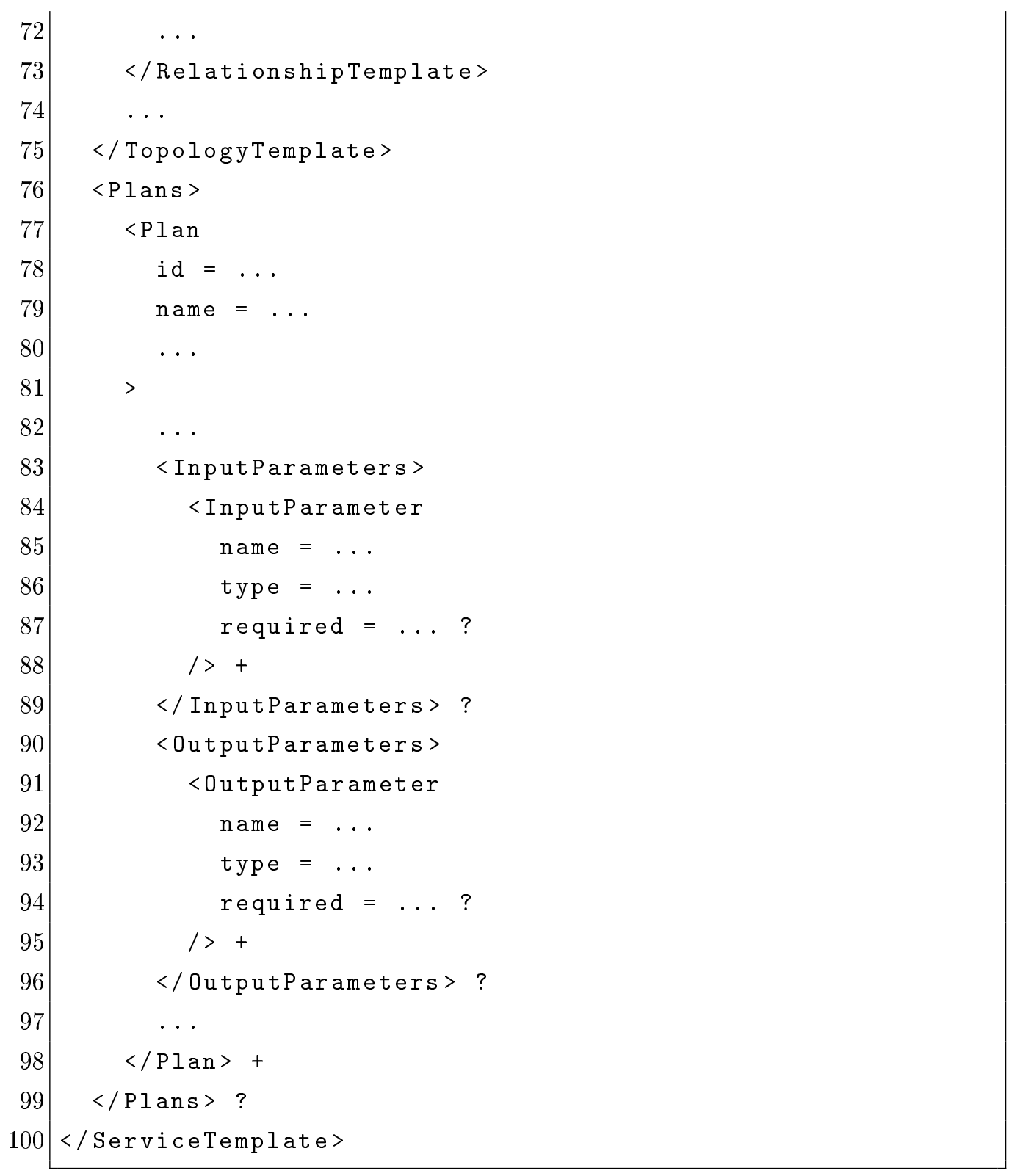

Listing 3.4: High level syntax of TOSCA NodeType and ServiceTemplate elements interested in interfaces matching.

To obtain the desired exact matching condition, we need to ensure that both the NodeType element and the Service Template element expose the same interfaces. In other words, we want that each NodeType interface contains the same operations of exactly one of the ServiceTemplate interfaces (and vice versa). Formally: 
Definition 3.7. Let $N$ be a NodeType and ST a ServiceTemplate. We say that

$$
\text { N.Interfaces } \equiv_{I} \text { ST.Interfaces }
$$

if and only if:

1. $\forall$ Interface $x \in N$.Interfaces $\exists$ ! Interface $y \in S T$.Interfaces:

$x$. name $=y$. name $\wedge$

$\forall$ Operation $i \in x \exists$ ! Operation $j \in y$ :

$$
i \equiv{ }_{O} j
$$

2. $\forall$ Interface $y \in S T$.Interfaces $\exists$ ! Interface $x \in N$.Interfaces:

$y$. name $=x$. name $\wedge$

$\forall$ Operation $j \in y \exists$ ! Operation $i \in x$ :

$$
j \equiv{ }_{O} i
$$

The above definition requires to specify how two Operation elements can be considered in the $\equiv_{O}$ relationship.

Definition 3.8. Consider two Operation elements $O_{1}$ and $O_{2}$. We say that

$$
O_{1} \equiv_{O} O_{2}
$$

if and only if

1. $O_{1}$.name $=O_{2}$. name

2. $\forall$ InputParameter $a \in O_{1}$.InputParameters

$\exists$ ! InputParameter $b \in \mathrm{O}_{2}$.InputParameters:

a.name $=$ b.name $\wedge$ a.type $=$ b.type $\wedge$ a.required $=$ b.required 
3. $\forall$ InputParameter $b \in O_{2}$.InputParameters

$\exists$ ! InputParameter $a \in O_{1}$.InputParameters

b.name $=$ a.name $\wedge$ b.type $=$ a.type $\wedge$ b.required $=$ a.required

4. $\forall$ OutputParameter $a \in O_{1}$.OutputParameters

$\exists$ ! OutputParameter $b \in O_{2}$. OutputParameters:

a.name $=$ b.name $\wedge$ a.type $=$ b.type $\wedge$ a.required $=$ b.required

5. $\forall$ OutputParameter $b \in \mathrm{O}_{2}$. OutputParameters

$\exists$ ! OutputParameter $a \in O_{1}$. OutputParameters:

b.name $=$ a.name $\wedge$ b.type $=$ a.type $\wedge$ b.required $=$ a.required

\subsubsection{Exact matching examples}

Suppose that our TOSCA cloud application requires a node whose type is the one in Figure 3.2. What we want to do is to check whether existing

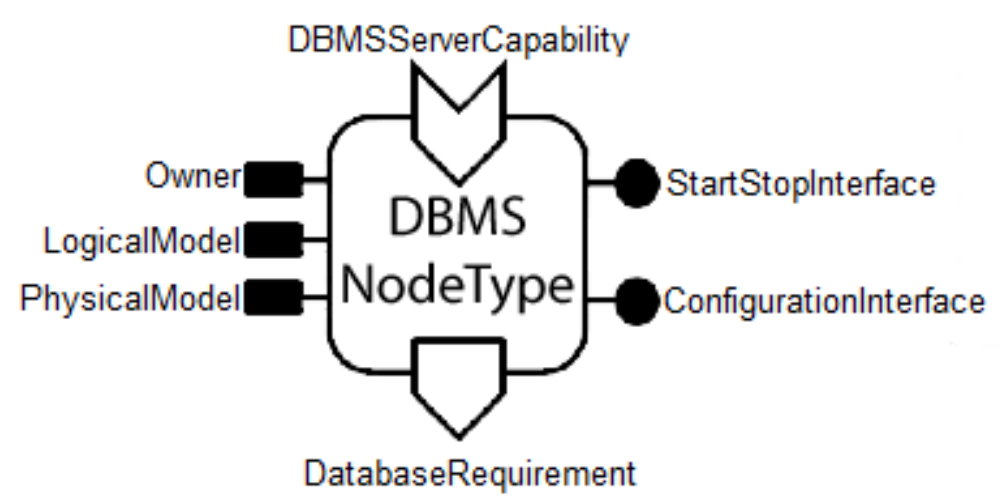

Figure 3.2: TOSCA DBMSNodeType example.

ServiceTemplate elements can be used as substitute for NodeTemplate elements of the specified NodeType. In the following paragraphs we will show two examples of existing Service Template to be matched ${ }^{2}$; the first

\footnotetext{
${ }^{2}$ For the sake of simplicity, we will consider ServiceTemplate elements not exposing any kind of Policy.
} 
one will be fully compatible with the desired NodeType; the second one will not.

Suppose that the ServiceTemplate $S T$ in Figure 3.3 is available and suppose that its Interface elements contains the same operations of the ones in the DBMSNodeType. It is clear that the following condition is

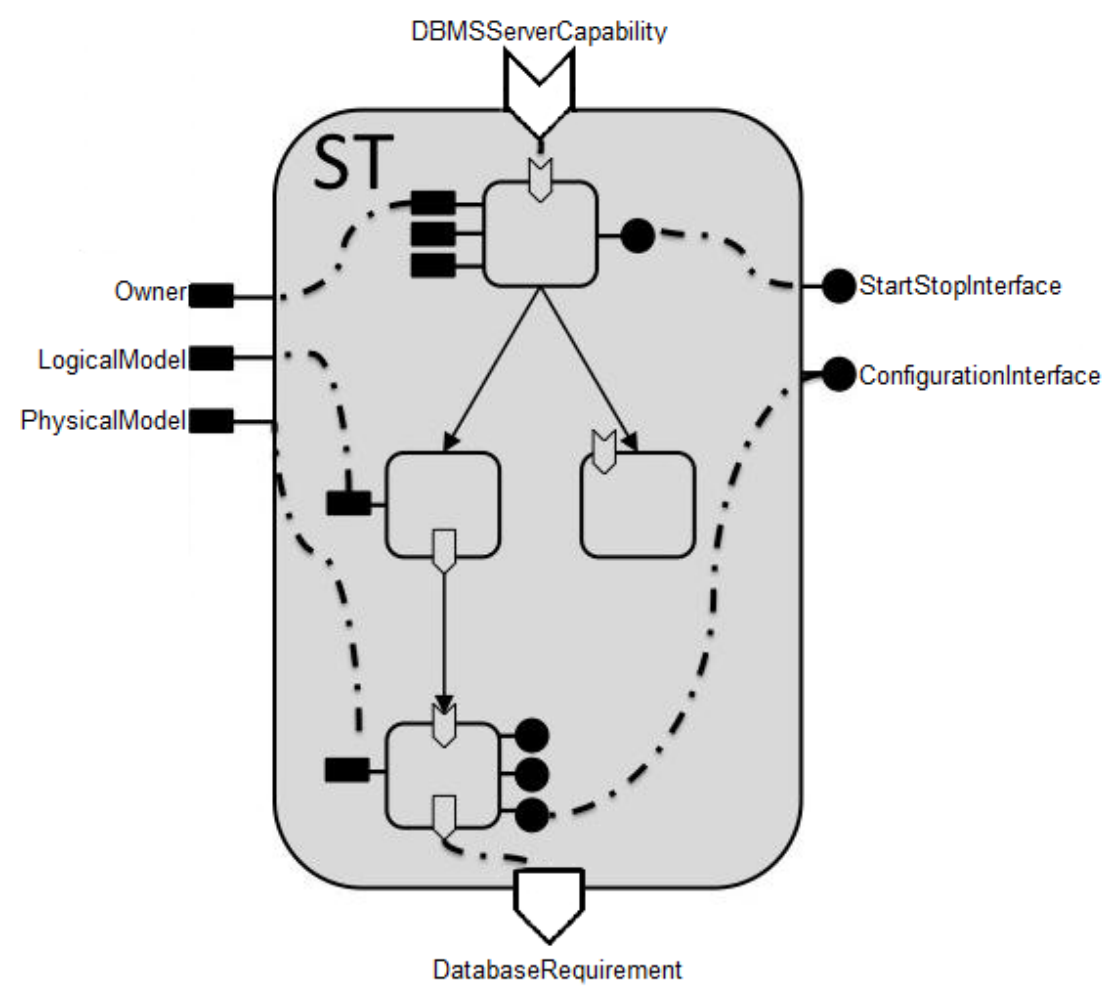

Figure 3.3: TOSCA ServiceTemplate exact matching example.

true:

$$
S T \equiv D B M S N o d e T y p e .
$$

Let us now modify the previous ServiceTemplate $S T$ by exposing just another property of the nested nodes (Figure 3.4). Because of the introduction of the new Property in $S T^{\prime}$ the exact matching condition becomes false. Nevertheless, it is clear that the ServiceTemplate under 


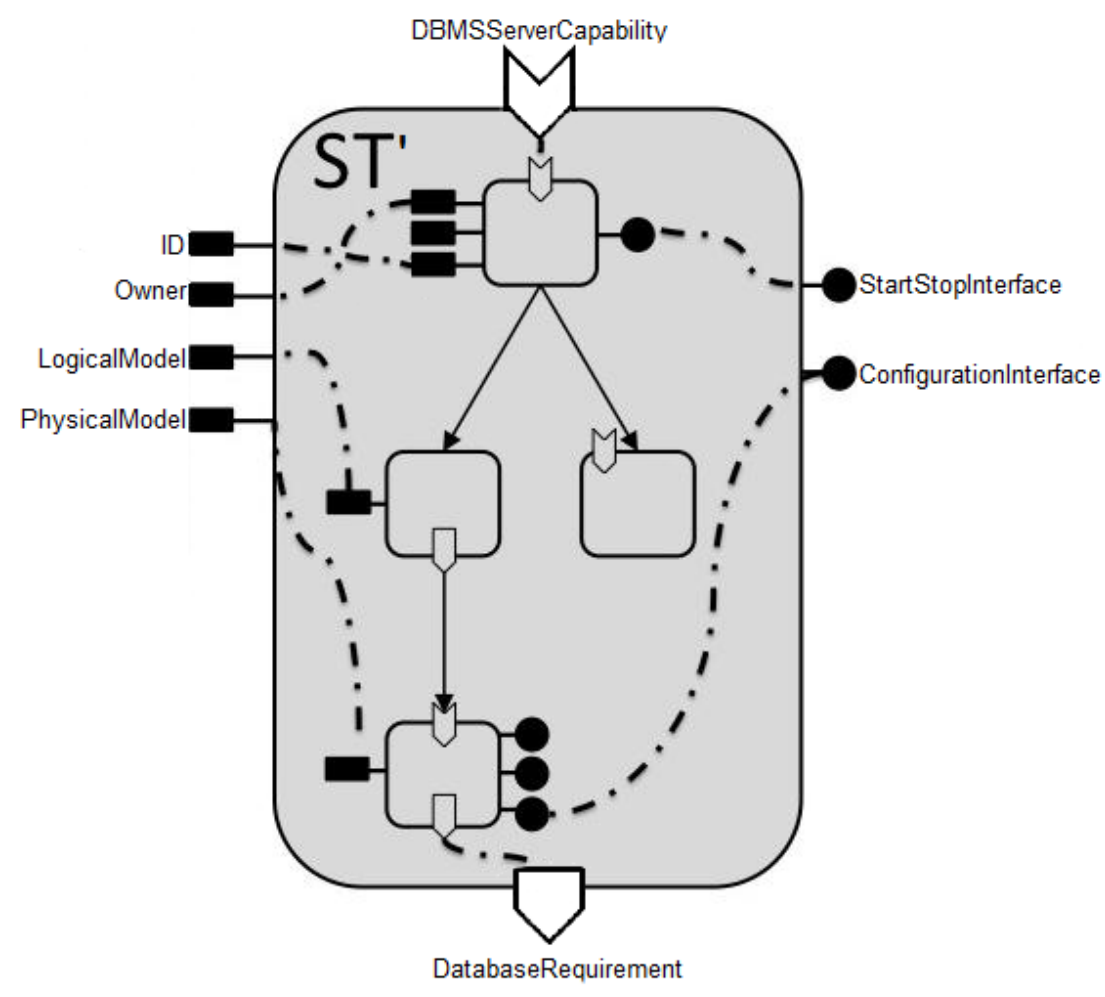

Figure 3.4: TOSCA ServiceTemplate exact matching example (modified). 
consideration (with some simply adaptation) can be used as a substitute for the DBMSNodeType.

The problem stays in the too much strict matching condition given in Definition 3.1. So, we need to be more flexible (by relaxing some of the constraints).

\subsection{Plug-in matching}

In the previous section we have provided a notion of full compatibility between NodeType and ServiceTemplate elements. Then, that notion has been applied to some examples. In the last one of these we have observed that, despite the ServiceTemplate element under consideration could be simply adapted to be compatible with the NodeType we want to match, the Definition 3.1 cannot be used. As previously reported, the problem stays in the too much strict conditions provided by the definition considered.

In this section we will relax some of the Definition 3.1 boundaries in order to obtain a formal definition of plug-in matching.

\subsubsection{Definition of plug-in matching}

The objective of this section is to define an operator " $\subseteq$ " analogue to the "三" introduced in Section 3.1: it takes a pair 〈NodeType,ServiceTemplate $\rangle$ and returns a truth value (which is true if the NodeType and ServiceTemplate are in plug-in matching, false otherwise).

To better understand how this operator works, we have to clarify what plug-in matching means. Consider the NodeType $N$ and the ServiceTemplate $S T$. Intuitively speaking, we say that $N \subseteq S T$ if:

- $S T$ 's policies are applicable to $N$, 
- $N$ exposes more requirements than $S T$, and

- $N$ offer less capabilities, properties and operations than $S T$.

If this is the case, then we can easily adapt $S T$ (without looking inside of it) in order to obtain a ServiceTemplate $S T^{\prime}$ such that $N \equiv S T^{\prime}$.

Observation 3.1. Please note that if $N \equiv S T$ then there is no need of adaptation on $S T$ to obtain the exact matching condition. This means that

$$
N \equiv S T \Longrightarrow N \subseteq S T
$$

Now we have all the fundamentals needed to proceed defining formally what plug-in matching means.

Definition 3.9. A NodeType $N$ plug-in matches a ServiceTemplate ST $(N \subseteq S T)$ if and only if:

$$
\begin{gathered}
\text { N.RequirementDefinitions } \subseteq_{R} \text { ST.Requirements } \wedge \\
\text { N.CapabilityDefinitions } \subseteq_{C} S T . \text { Capabilities } \wedge \\
\text { PolicyType applicable to } N \equiv_{P O} \text { ST.Policies } \wedge \\
\text { N.PropertiesDefinition } \subseteq_{P R} \text { ST.Properties } \wedge \\
\text { N.Interfaces } \subseteq_{I} \text { ST.Interfaces }
\end{gathered}
$$

As before, to better understand what the above definition means, we have to focus on each of the given conditions ${ }^{3}$.

\footnotetext{
${ }^{3}$ Please note that, since a NodeType $N$ elements cannot expose policies, we still have to check whether ServiceTemplate's policies are applicable to $N$ (as it was in Definition 3.1.
} 
Chapter 3. NodeType black-box matching (and adaptation)

\section{Plug-in matching of requirements}

A ServiceTemplate $S T$ can be used as a substitute for NodeType $N$ if it exposes less requirements than $N$. In other words, if $S T \subseteq N$ then the set of $S T$ requirements is a subset of the set of $N$ requirements.

Looking at TOSCA specification [25] we observe that

- the semantics of the requirement only depends on its type. It follows that during requirements matching there is no need to consider the name attribute;

- a RequirementType can inherit semantics from another RequirementType by means of the DerivedFrom element. This force us to consider inheritance during our matching.

Observe that, if a RequirementType $R T$ is derived from a RequirementType $R T^{\prime}$ then $R T$ extends the semantics of $R T^{\prime}$ (e.g. the WebServerRequirementType can be extended with the ApacheWebServerRequirementType; the former only requires a web server to be satisfied; the latter requires a web server with Apache software to be satisfied). It follows that, if we want to substitute the NodeType $N$ with the ServiceTemplate ST, then the requirement types of $S T$ must be the same or super-types of the one of $N$.

So, before giving the desired formal condition, we have to define an operator to indicate that a TOSCA element is derived from another one.

Definition 3.10. The infix operator $\vdash$ takes a pair of TOSCA elements $T_{1}$ and $T_{2}$ and returns a truth value according to the following rules:

- $T_{1} \vdash T_{2}=$ true, if $T_{1}$. DerivedFrom contains a reference to $T_{2}$;

- $T_{1} \vdash T_{2}=$ true, if $T_{1}$. DerivedFrom contains a reference to a TOSCA element $T_{3}$ such that $T_{3} \vdash T_{2}$; 
- $T_{1} \vdash T_{2}=$ false, otherwise.

Definition 3.11. Let $N$ be a NodeType and ST be a ServiceTemplate. We say that

\section{N.RequirementDefinitions $\subseteq_{R}$ ST.Requirements}

if and only if:

$\forall$ Requirement $y \in S T$.Requirements

$\exists$ RequirementDefinition $x \in N$.RequirementDefinitions:

x.ref.type $=$ y.requirementType $\vee$

x.requirementType $\vdash$ y.ref.type.

\section{Plug-in matching of capabilities}

As before, the high correlation between capabilities and requirements TOSCA syntax lets us do the same reasoning and taking the same conclusions. There is only one difference between the two cases: this time we talk about what $S T$ offers (and not what it requires). So, this time the inclusion relationship is between the set of $N$ capabilities and the set of $S T$ capabilities. Formally:

Definition 3.12. Let $N$ be a NodeType and $S T$ be a ServiceTemplate. We say that

\section{N.CapabilityDefinitions $\subseteq_{C}$ ST.Capabilities}

if and only if: 
Chapter 3. NodeType black-box matching (and adaptation)

\author{
$\forall$ CapabilityDefinition $x \in N$.CapabilityDefinitions \\ $\exists$ Capability $y \in$ ST.Capabilities: \\ y.ref.type $=x$.requirementType $\vee$ \\ y.ref.type $\vdash x$.requirementType.
}

\title{
Plug-in matching of properties
}

Talking about exact matching, we sad that the properties XML type of NodeType $N$ and ServiceTemplate ST must be the same. Observe that this a too strict condition: we only need that $S T$ offers at least the same properties as $N$. Indeed, if $S T$ offers more properties than $N$, we can hide the exceeding ones (in order to obtain the exact matching).

Definition 3.13. Let $N$ be a NodeType and $S T$ be a ServiceTemplate. We say that

\section{N.PropertiesDefinition $\subseteq_{P R}$ ST.Properties}

if and only if the XML type of ST.Properties extends the one defined via N.PropertiesDefinition.

\section{Plug-in matching of interfaces}

In the exact matching reasoning, we want that the ServiceTemplate ST offers the same interfaces as NodeType $N$. This is a too coarse grain reasoning: we only need that for each operation $O$ exposed by $N$ exists at least one of the $S T$ operations which is equal to $O$. If this is the case, then we can group operations in order to obtain the desired interfaces. 
This finer grain reasoning let us give the formal interfaces plug-in matching condition.

Definition 3.14. Let $N$ be a NodeType and ST be a ServiceTemplate. We say that

$$
N . \text { Interfaces } \subseteq_{I} \text { ST.Interfaces }
$$

if and only if:

$$
\begin{gathered}
\forall \text { Operation } x \in \text { N.Interfaces.Interface, } \\
\exists \text { Operation } y \in \text { ST.Interfaces.Interface: } \\
x \equiv_{O} y
\end{gathered}
$$

Observation 3.2. Please note that the operation exposed by $N$ and the one exposed by $S T$ sill must be in a exact match $\left(\equiv_{O}\right)$ relationship.

\subsubsection{Adaptation: the oblivion boundaries approach}

In the previous section we have seen a plug-in manner to check whether a ServiceTemplate can be a substitute for a NodeType. It is worth noting that such a flexibility requires the introduction of a kind of service adaptation to let the ServiceTemplate be used in place of the NodeType.

Remember that we are facing the problem with a black-box approach. So, we cannot modify the ServiceTemplate internally: we can only operate out of its boundaries. To show how this external adaptation is done, we will start looking at what the NodeType offers (CapabilityDefinitions, PropertiesDefinition and Interfaces). Once the offerings adaptation is 
done we will consider also the adaptation of what the NodeType needs (RequirementDefinitions) ${ }^{4}$.

\section{Capabilities, properties and interfaces adaptation}

The idea of plug-in matching is to check whether a ServiceTemplate ST offers at least the same capabilities, properties and operations as the NodeType $N$ we need to substitute. If this is the case, the next step is to restrict the set of TOSCA elements offered by $S T$ to only those offered by $N$.

The desired adaptation can be done by constructing a new Service Template $S T^{\prime}$ via the oblivion boundaries approach (Figure 3.5. The

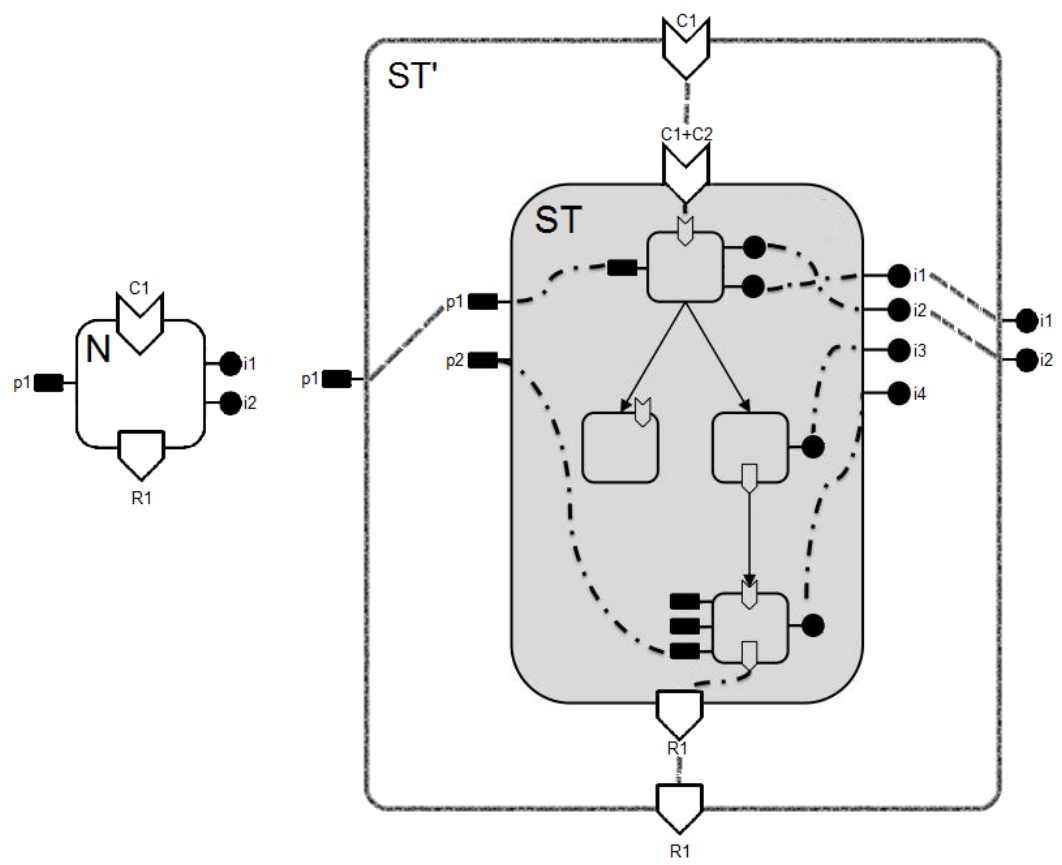

Figure 3.5: Oblivion boundaries adaptation approach.

new service TopologyTemplate will be (by now) composed by only one

\footnotetext{
${ }^{4}$ Since we still check whether policies are exactly matched, we do not need to adapt them.
} 
Chapter 3. NodeType black-box matching (and adaptation)

node: the ServiceTemplate ST we want to adapt. Once the topology is defined, we have to decide what to offer externally (via the BoundaryDefinitions element):

- $S T^{\prime}$ will expose only the properties defined in N.PropertiesDefinition element;

- the set of capabilities exposed by $S T^{\prime}$ will be the subset of $S T$ capabilities in plug-in matching with the ones of $N$. Because of possible name mismatchings, the capabilities are renamed (if needed) in order to be the same as the ones exposed by $N$;

- for each interface of $N$, the required operations are detected and grouped to form the relative interface of $S T^{\prime}$.

\section{Full adaptation}

Let us also consider requirements in our adaptation procedure. Remember that a ServiceTemplate $S T$ is compatible with a NodeType $N$ only if the latter exposes more requirements than the former. This clearly means that this time we cannot restrict what $S T$ exposes to what $N$. Indeed, we have to transform $S T$ in $S T^{\prime}$ making such a kind of requirements addition. How can this be done?

In the previous paragraph, while we were talking about the oblivion boundaries adaptation approach (without requirements adaptation consideration), we said that we have to create a new service $S T^{\prime}$ in which $S T$ is the only NodeTemplate. To compensate missing requirements we can introduce another node in our topology: the echo node (see Figure 3.6. This node has no functional meaning: its only purpose is to replicat 5 $S T$ requirements and add missing requirements. With the echo node, our new service $S T^{\prime}$ can expose all the requirements declared by $N$. This let us say $S T^{\prime} \equiv N$ and then $S T^{\prime}$ can be a candidate for $N$ substitution.

\footnotetext{
${ }^{5}$ This replication justifies the name echo.
} 


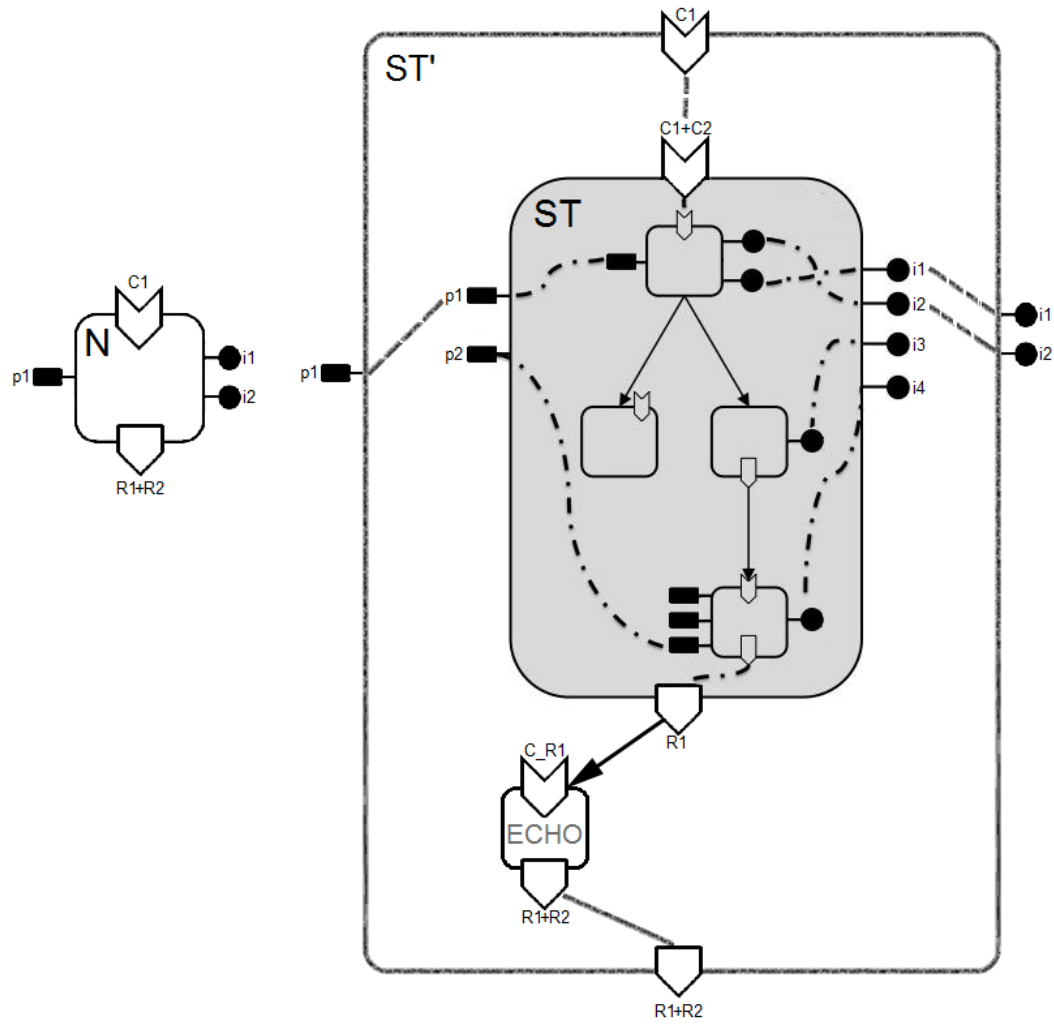

Figure 3.6: Echo node usage in the oblivion boundaries adaptation approach. 
Chapter 3. NodeType black-box matching (and adaptation)

\section{Concluding remark}

Before concluding the discussion of the oblivion boundaries approach, we have to make some important practical observation.

Because of our adaptation approach only requires to restrict the available service boundaries and (eventually) add some new requirements, one could think of simplifying the $S T$ boundaries so as to make them equal to those desired. Is it a right approach? Let us think about what to transform the $S T$ specification in the $S T^{\prime}$ one implies. If we change that specification, then we have to develop a new cloud service application (which is clearly an extremely expensive approach). So, it is important to maintain the $S T^{\prime}$ specification as an adaptation of $S T$ since this is a valuable information for deploying the needed adaptation.

Consider now the situation in which $N$ is a NodeType required by a client and $S T$ is a Service Template offered by a provider. Once $S T^{\prime}$ specification has been defined, the client and the provider could behave as follows:

1. the client still interacts with $S T$ without considering the oversupplied features. If this is the case, no adaptation is implemented (and the provider believes that the client does not use what she has not required);

2. the client interacts with a cloud service application implementing $S T^{\prime}$ (offered by the provider). Typically, this application is obtained by generating an adaptor for the available application implementing $S T$.

\subsubsection{Plug-in matching (and adaptation) examples}

Let us reconsider the matching example of Figure 3.4. With the too strict exact matching conditions the compatibility checking fails. Our 
new plug-in matching (and oblivion boundaries adaptation) approach instead makes that checking be satisfied.

A more complete example of plug-in matching can be done with the situation in Figure 3.7. In this case we want to match an available ServiceTemplate $S T$ with a NodeType $N$. The NodeType under consideration has the following characteristics:

- it offers less properties and interfaces ${ }^{6}$ than $S T$;

- it offers the same capabilities as $S T$;

- it needs a requirement (ApacheApplicationContainerRequirement) which type is derived from the one of the requirement exposed by ST (ApplicationContainerRequirement).

With this configuration we can easily verify the truth of the following condition:

$$
\begin{gathered}
\text { N.CapabilityDefinitions } \equiv_{C} \text { ST.Capabilities } \wedge \\
\text { N.RequirementDefinitions } \subseteq_{R} \text { ST.Requirements } \wedge \\
\text { N.PropertiesDefinition } \subseteq_{P R} \text { ST.Properties } \wedge \\
\text { N.Interfaces } \subseteq_{I} \text { ST.Interfaces. }
\end{gathered}
$$

In other words, we can easily check that $S T \subseteq N$. This means that we can adapt the service $S T$ in order to obtain a service $S T^{\prime}$ which exact matches $N$ (see Figure 3.8).

Let us now modify the ServiceTemplate of Figure 3.7.(b) as reported in Figure 3.9. Suppose that:

- the GetStatistics interface of $N$ (see Figure 3.7.(a)) contains the only Get operation;

\footnotetext{
${ }^{6}$ For the sake of simplicity, we are assuming that Interface elements with the same name are offering the same Operation elements.
} 


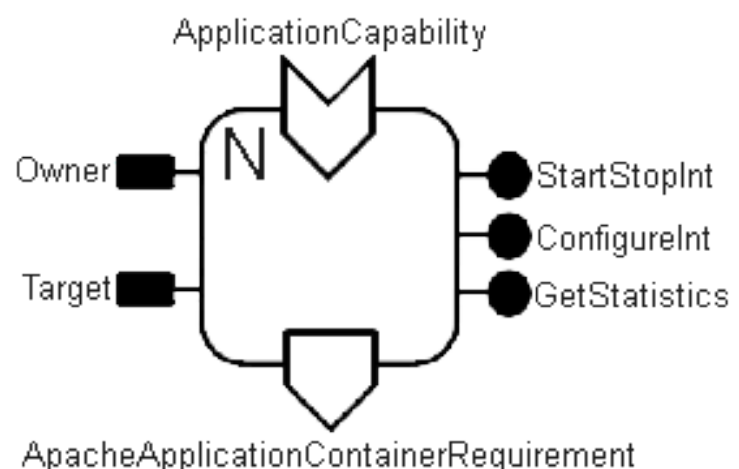

(a)

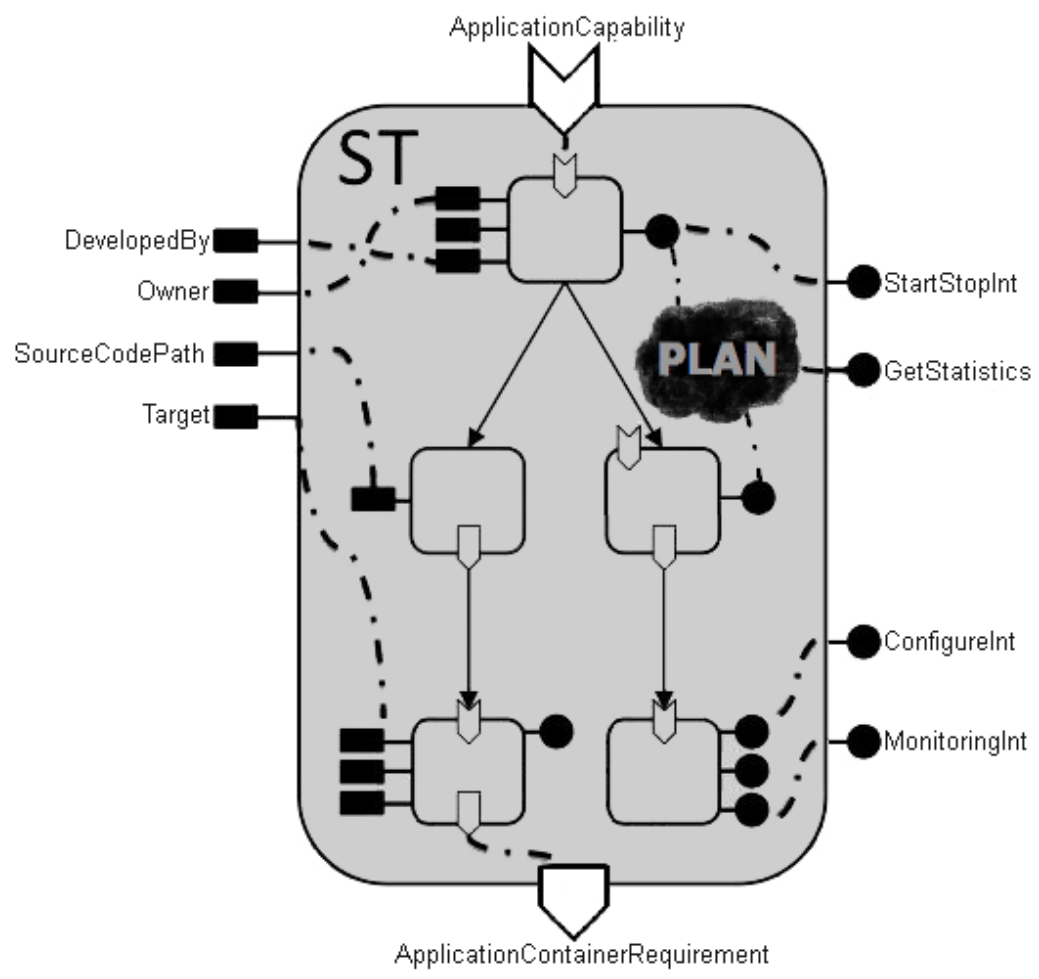

(b)

Figure 3.7: TOSCA ServiceTemplate plug-in matching example. 


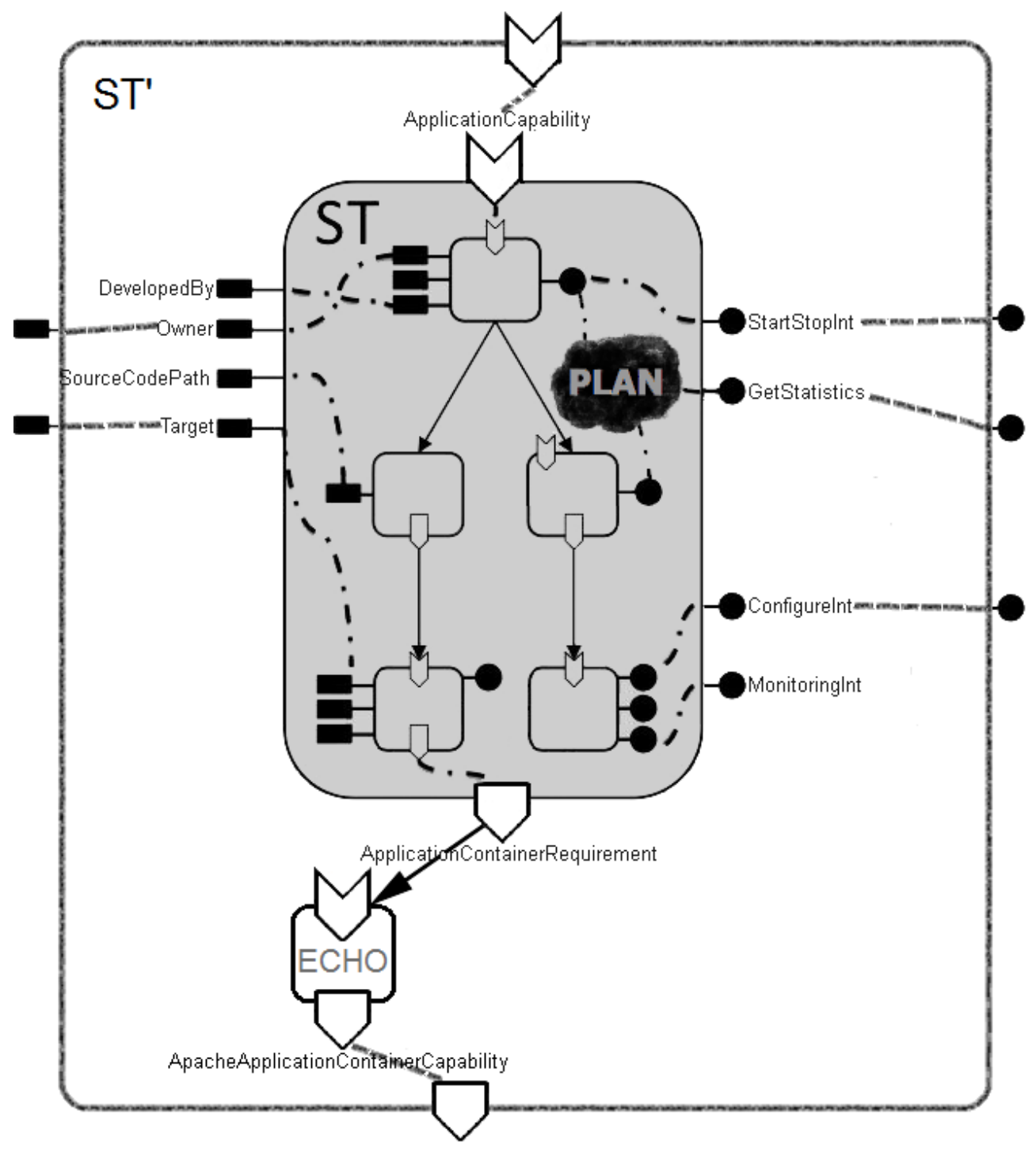

Figure 3.8: TOSCA ServiceTemplate oblivion boundaries adaptation example. 


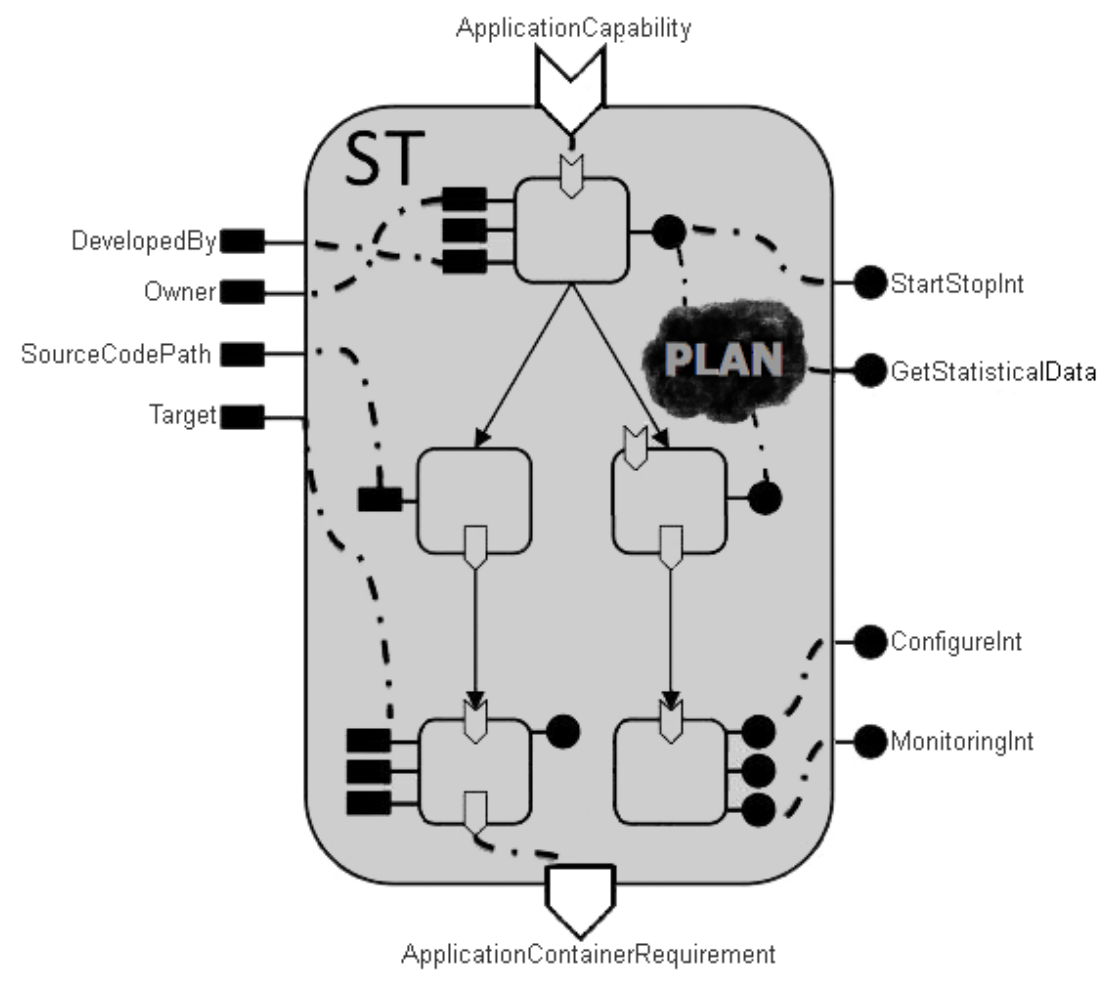

Figure 3.9: TOSCA ServiceTemplate plug-in matching example (modified). 
- the GetStatisticalData interface of ST contains the only GetData operation (which is semantically equivalent ${ }^{7}$ to Get).

Clearly, with the $\subseteq$ operator, $N$ cannot be considered compatible with $S T$. So, despite the two operation are semantically equivalent, the compatibility check fails.

How can we deal with this problem? So far we have worked at a syntactic level. Introducing such a kind of semantics will let us overcome problems such as the one previously indicated.

\subsection{Flexible matching}

With the examples given in Section 3.2 we have shown how the purely syntactical matching (defined by the operator " $\subseteq$ ") could not be enough to check whether a NodeType element can be substituted by a ServiceTemplate element.

The objective of this section is to overcome the exposed problem using some kind of semantic checking. Please note that, since:

- NodeType elements cannot specify any kind of policy, and

- requirements (capabilities) semantics is specified ${ }^{8}$ via their RequirementType (Capability Type),

the semantic check only affects properties and interface operations.

\footnotetext{
${ }^{7}$ With semantically equivalent we mean that it requires the same input parameters and produces the same output parameters.

${ }^{8}$ As TOSCA [25] authors says.
} 
Chapter 3. NodeType black-box matching (and adaptation)

\subsubsection{Definition of flexible matching}

What we are now going to do is to define a new operator "" analogue to

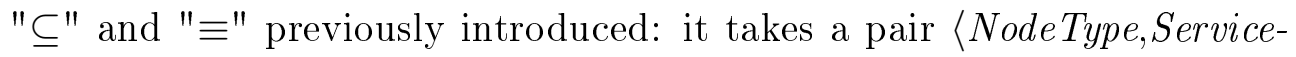
Template $>$ and returns a truth value (which is true if the two input elements are in flexible matching, false otherwise).

Understanding how the new operator works requires to clarify what flexible matching means. Consider the NodeType $N$ and the ServiceTemplate $S T$; we say that $N \cong S T$ if the plug-in matching fails only because of the presence of operations and/or properties which are syntactically different but semantically equivalent. So, formally, we only have to modify the " $\subseteq$ " conditions about interfaces and properties.

Definition 3.15. A NodeType $N$ flexibly matches a ServiceTemplate $S T$ $(N \cong S T)$ if and only if:

$$
\begin{gathered}
\text { N.RequirementDefinitions } \subseteq_{R} \text { ST.Requirements } \wedge \\
\text { N.CapabilityDefinitions } \subseteq_{C} \text { ST.Capabilities } \wedge \\
\text { PolicyType applicable to } N \equiv_{P O} \text { ST.Policies } \wedge \\
\text { N.PropertiesDefinition } \cong_{P R} \text { ST.Properties } \wedge \\
\text { N.Interfaces } \cong_{I} \text { ST.Interfaces }
\end{gathered}
$$

Observation 3.3. Please note that, if two properties (or interfaces) are syntactically (and semantically) equivalent, then they are both plug-in and flexibly matched. This intuitively means that the following property holds:

$$
N \subseteq S T \Longrightarrow N \cong S T
$$

Let us clarify what the new properties and interfaces matching conditions means. 
Chapter 3. NodeType black-box matching (and adaptation)

\section{Required assumptions}

Before giving the desired flexible matching conditions, we have to introduce some concerns about semantics.

In the following, we will use ontologies to associate semantic meaning to TOSCA elements under consideration. This force us to make the following assumption.

Assumption 3.2. All cloud service applications are equipped with ontologies (which associate semantic meaning to all TOSCA element names used in their definitions).

This let us assume that the NodeType and the ServiceTemplate we want to match have semantics associated to their names. So, we can proceed in checking whether their elements' semantic meaning is the same. But, how can we perform such a checking?

Assumption 3.3. Suppose that a cross-ontology matchmaker $C O M$ is available. This matchmaker let us verify whether two different ontologies concepts have equivalent semantic meanings.

In the following we will use the notation $C_{a} \vdash \dashv C_{b}$ to indicate the semantic checking (done by $C O M$ ) between different ontologies concepts $C_{a}$ and $C_{b}$, where $C_{a} \vdash \dashv C_{b}$ if and only if they are semantically equivalent.

\section{Flexible matching of properties}

The Definition 3.13 says that N.PropertiesDefinition plug-in matches ST.Properties if and only if the XML type of the latter extends the one of the former. In other words, each property $P_{N}$ defined in the N.PropertiesDefinition element must correspond to a property $P_{S T}$ of the ServiceTemplate such that:

- $P_{S T}$.type is (the same as or) a sub-type of $P_{N}$.type, and 
- $P_{S T}$.name is equal to $P_{N}$.name.

If the first condition is not satisfied, then there are no chances to adapt the ServiceTemplate $S T$ to make it be compatible with $N$. Vice versa, if two properties do not have the same name, then we can check if they are semantically equivalent.

Definition 3.16. Let $N$ be a NodeType and $S T$ be a ServiceTemplate. We say that

\section{N.PropertiesDefinition $\cong_{P R}$ ST.Properties}

if and only if

$$
\begin{gathered}
\forall \text { propertyDefinition } x \in N \text {.PropertiesDefinition, } \\
\exists \text { property } y \in S T \text {.Properties: }
\end{gathered}
$$

the XML type of $y$ extends the one defined by $x \wedge$

$$
\text { y.name } \vdash \dashv x \text {.name }
$$

\section{Flexible matching of interfaces}

Let us now consider the operations (and interfaces) flexible matching problem. What we want to do is to let an operation $O_{1}$ be substituted by another operation $\mathrm{O}_{2}$ which can be considered semantically equivalent. This semantic equivalence can be explained as follows:

- the $O_{1}$ input (output) parameters number is the same as the one of $\mathrm{O}_{2}$;

- for each $O_{1}$ input (output) parameter exists a $O_{2}$ input (output) parameter of the same type which name is semantically equivalent to the one of $O_{1}$; 
Definition 3.17. Consider two Operation elements $O_{1}$ and $O_{2}$. We say that

$$
O_{1} \cong_{O} O_{2}
$$

if and only if the following conditions are satisfied:

1. $\mid\left\{O_{1}\right.$.InputParameters $\}|=|\left\{O_{2}\right.$.InputParameters $\} \mid$

2. $\forall$ InputParameter $b_{\text {in }} \in O_{2}$.InputParameters

$\exists$ InputParameter $a_{\text {in }} \in O_{1}$. InputParameters:

$$
a_{\text {in. }} \text {.name } \vdash \dashv b_{\text {in. }} \text {.name } \wedge a_{\text {in }} \text {.type } \vdash b_{\text {in }} \text {.type }
$$

3. $\mid\left\{O_{1}\right.$. OutputParameters $\}|=|\left\{O_{2}\right.$.OutputParameters $\} \mid$

4. $\forall$ OutputParameter $a_{\text {out }} \in O_{1}$. OutputParameters

$\exists$ OutputParameter $b_{\text {out }} \in O_{2}$. OutputParameters:

$$
b_{\text {out }} \text {.name } \vdash \dashv a_{\text {out }} \text {.name } \wedge b_{\text {out }} \text {.type } \vdash a_{\text {out }} \text {.type }
$$

So, we have all the fundamentals needed to define the flexible matching condition between NodeType and ServiceTemplate interfaces.

Definition 3.18. Let $N$ be a NodeType and $S T$ be a ServiceTemplate. We say that

$$
N . \text { Interfaces } \cong_{I} \text { ST.Interfaces }
$$

if and only if:

$$
\begin{gathered}
\forall \text { Operation } x \in \text { N.Interfaces.Interface } \\
\exists \text { Operation } y \in \text { ST.Interfaces.Interface: } \\
x \cong_{O} y
\end{gathered}
$$




\section{Concluding remark}

Note that (by using the " $\vdash$ " notation) we abstract from how the semantic checking is done. To do it we can use one of the already available cross-ontology matchmakers. In other words, according to the purpose of this thesis, in the following we will not consider the problem of realizing a cross-ontology matchmaker but we will consider the usage of one already available (such as the one proposed by Martinez-Gil et al. [20]).

\subsubsection{Adaptation: extended oblivion boundaries ap- proach}

In the previous subsection we have seen how it is possible to use ontological semantics in order to obtain the flexible matching. What we have done is simply modify the properties and interfaces plug-in matching (done by the " $\subseteq$ " operator) introducing the semantic checking. Similarly, modifying properties and interfaces adaptation, we can reuse the oblivion boundaries adaptation approach.

Observe that the only (but fundamental) checking modification is in the possibility for properties and operations to have different names. So, simply introducing the ServiceTemplate properties and operation renaming (in order to match what the NodeType exposes), the oblivion boundaries can be reused.

\subsubsection{Flexible matching (and adaptation) examples}

Reconsider the failed example of Section 3.2 (Figure 3.9). With the plugin matching (and adaptation) approach our checking fails because of the presence of operations which names are different. Assume that:

- the operations Get and GetData do not have any input parameter, 
and

- Get and GetData expose the same number of output parameters (and those parameters name are semantically equivalent).

Under the above assumptions the condition

$$
G e t \cong_{O} \text { GetData }
$$

is true. This means that we can easily verify that $N \cong S T$. So, we can adapt the ServiceTemplate $S T$ in order to match $N$ with the (extended) oblivion boundaries approach.

\subsection{Concluding remarks}

In this chapter we have seen three different ways to black-box match a NodeType $N$ with a ServiceTemplate ST. Before going on with our discussion, we have to make some observations.

\subsubsection{Matching and adaptation}

As reported in Figure 3.10, the proposed black-box matching (and adaptation) approach can be fully automated in order to generate the adapted $S T^{\prime}$ ServiceTemplate. In other words, we can:

- check whether (and how) the two TOSCA elements under consideration match, and

- (possibly) generate the desired $S T$ adaptation.

Looking at Figure 3.10 , we observe that, if one of the proposed black-box matching conditions is satisfied, then we can generate an adapted service 


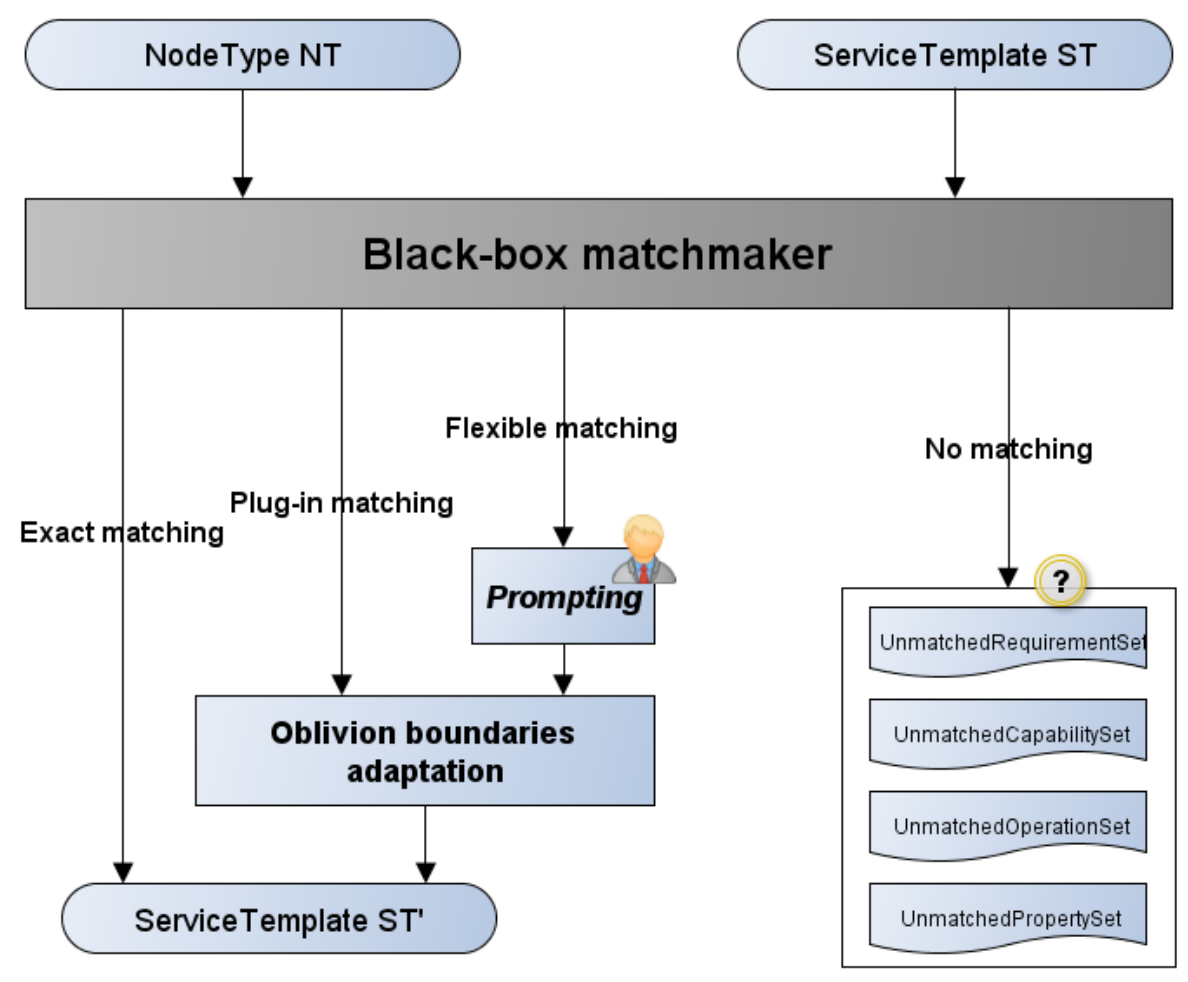

Figure 3.10: Black-box matching (and adaptation) procedure. 
$S T^{\prime}$. This means that we always follow the approach of Figure 3.11. instead of developing a new service, we use a (automatically generated) adaptor $A$ to interact with the available service $S T$ as if it were the desired $S T^{\prime}$.

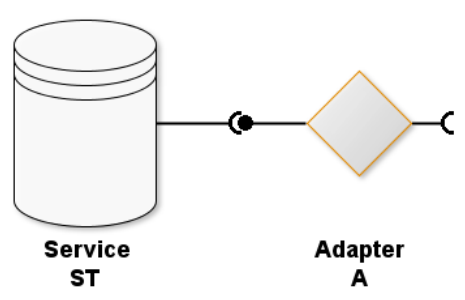

Figure 3.11: Adaptation approach.

The above considerations suggest us a question: which kind of adaptation does $A$ have to implement? To answer it we have to distinguish the possible matching cases. Consider, as always, the ServiceTemplate ST and the NodeType N.

Exact matching If $N \equiv S T$, then $S T$ exposes exactly what $N$ needs (and so there is no need of adapting ST). This means that, in order to let $S T^{\prime}$ be the same service as $S T$, the generated adaptor $A$ must implement the identity function.

Plug-in matching If $N \subseteq S T$, then $S T$ exhibits all the features needed by $N$ and some features that $N$ does not need. If this is the case, then we have to restrict what $S T$ exposes to only what $N$ needs. In other words, the function that $A$ has to implement is a filtering one.

Flexible matching The last matching case $(N \cong S T)$ means that $S T$ exposes

- features that are equivalent to all those required by $N$ (possibly with different names), and 
- some undesired features.

This implies that the adaptor $A$ must implement a function which filters and (possibly) renames those features.

\subsubsection{Taking semantics into account}

When we talked about the flexible matching approach, we stated that ontologies should be used to check whether two features could be considered (semantically) equivalent. So, the generated pairings between (semantically) equivalent features strictly depend on the given ontologies and on the cross-ontology matchmaker. It follows that, if the input ontologies are not so accurate as required, then some generated pairings could not be significant and should not be used in our matching (and adaptation). How can we deal with this problem?

Looking at Figure 3.11 we can observe that when the considered NodeType $N$ and ServiceTemplate $S T$ are in flexible matching, we have to prompt to the user the generated pairings. If she accepts the matchmaker decisions, then we can proceed in developing the adapted service $S T^{\prime}$ (via the oblivion boundaries adaptation approach).

Please note that the way in which this prompting is performed strictly depends on the matchmaker implementation. We recommend to start giving the most probable pairings and to let the user mark and/or adjust the wrong pairings.

\subsubsection{Treatment of mismatchings}

We are studying the matching problem between a NodeType $N$ and a ServiceTemplate ST. So far, we have introduced a methodology to check whether those two TOSCA elements are in (one of the possible) black-box matching. Clearly, there are cases in which $N$ and $S T$ do not black-box 
Chapter 3. NodeType black-box matching (and adaptation)

match. So, what can we do when none of the exposed conditions is satisfied? We will see how to proceed in the following chapter. 


\section{Chapter 4}

\section{Node Type white-box matching (and adaptation)}

The previous chapter has provided an (automatable) approach which let us check whether a NodeType $N$ matches a ServiceTemplate ST (without looking "inside" $S T$ ). In this chapter we are going to see whether, using a white-box viewpoint, we can extend our matching procedure.

The chapter will start illustrating some examples which make the black-box matching procedures fail (Section 4.1). Then, it will proceed providing the white-box matching (and adaptation) approach (Sections 4.2 and 4.3). Finally, with Section 4.4, some concluding remarks are given.

\subsection{Motivating example}

Let us consider the ServiceTemplate ST in Figure 4.1. Suppose now we want to match it with the NodeType elements in Figure 4.2. Using the black-box matching conditions our checking fails because both WAppType 1 and WAppType 2 expose more TOSCA elements than ST. 


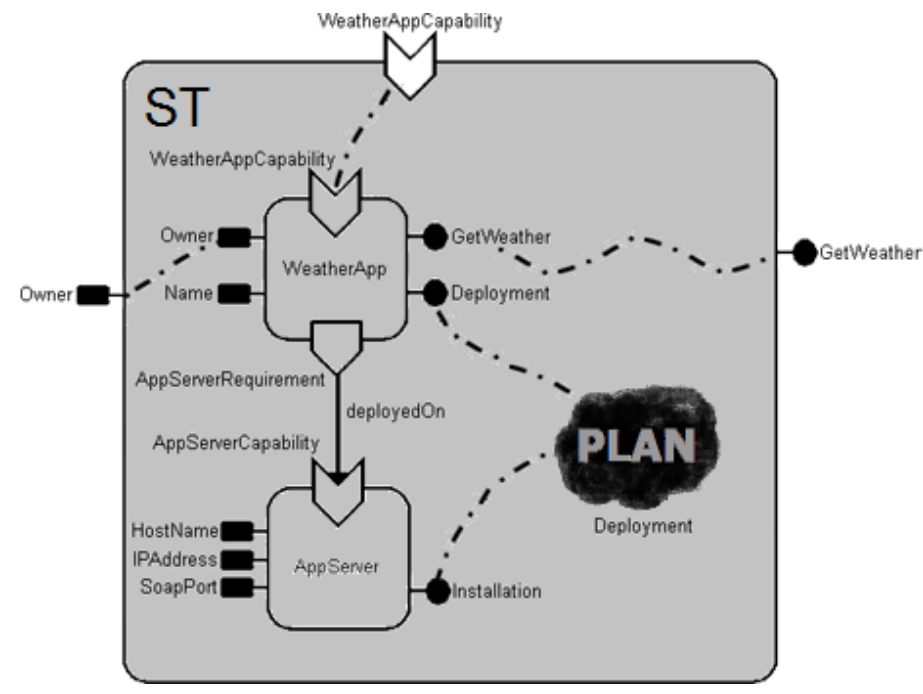

Figure 4.1: Example of available ServiceTemplate.

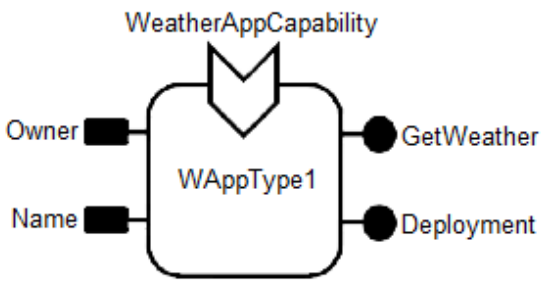

(a)

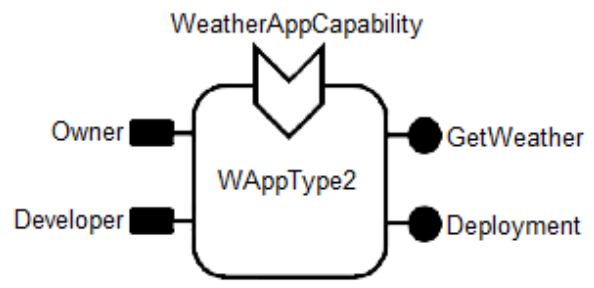

(b)

Figure 4.2: Example of desired NodeType elements. 
Let us now try to use a white-box approach. Looking inside $S T$ we can easily check that:

- the WAppType2 NodeType element cannot be matched with ST (because of the lack of Developer property presence);

- ST contains the Name (equivalent) property and the Deployment operation required by $W A p p T y p e 1$. More precisely, the Deployment operation can be obtained by generating a plan which combines available operations.

So, if we could find the missing features inside the available ServiceTemplate, then we could extract them in order to make our service black-box match the desired NodeType.

\subsection{White-box matching (and adaptation)}

Please reconsider Figure 3.10. Looking at the "no matching" case, we observe that our matching procedure could provide us the set of unmatched features (more precisely, those features could be given as a multiset which contains the UnmatchedRequirementSet, the UnmatchedCapabilitySet, the UnmatchedOperationSet and the UnmatchedPropertySet).

As observed in the previous section, we should find a way to exhibit all the unmatched required features via the boundaries of the available ServiceTemplate (if possible). This means that for each unmatched feature under consideration we should perform two steps:

1. Matching - an equivalent feature (if present) must be detected inside $S T$;

2. Adaptation - the boundaries of $S T$ must be modified in order to expose the desired feature. 
If this can be done, then we can reuse the black-box flexible matching approach in order to obtain the desired adaptation of the available Service Template.

Observation 4.1. Please note that (as done for flexible matching and adaptation), we have to use some kind of semantics in order to couple desired and available features. This means that we have to reconsider the following (ontology-about) assumptions.

Assumption 4.1. All cloud service applications are equipped with ontologies (which associate semantic meaning to all TOSCA element names used in their definitions).

Assumption 4.2. Suppose that a cross-ontology matcher $C O M$ is available. This matcher let us verify whether two different ontologies concepts have equivalent semantic meanings.

The above considerations give us an informal (though incomplete way of matching in a white-box viewpoint. In the rest of this section we will try to formalize this notion.

\subsubsection{White-box matching condition}

The objective of this subsection is to define a new operator " $\cong$ " analogue to those introduced for the black-box matching (Chapter 3). This new operator takes a pair 〈NodeType,ServiceTemplate〉 and returns a truth value. More precisely, it returns true if the two input elements are in white-box matching, false otherwise.

As done before, we can define the new operator meaning in a step-wise way.

Definition 4.1. A NodeType $N$ white-box matches a ServiceTemplate $S T(N \cong S T)$ if and only if:

\footnotetext{
${ }^{1}$ As we will see, dealing with unmatched operations treatment is more complex than simply searching (and exposing) the feature.
} 
Chapter 4. NodeType white-box matching (and adaptation)

\author{
N.RequirementDefinitions $\subseteq_{R}$ ST.Requirements $\wedge$ \\ N.CapabilityDefinitions $\cong_{C}$ ST.Capabilities $\wedge$ \\ PolicyType applicable to $N \equiv_{P O} S T$.Policies $\wedge$ \\ N.PropertiesDefinition $\cong_{P R}$ ST.Properties $\wedge$ \\ $N$.Interfaces $\cong_{I}$ ST.Interfaces
}

Observation 4.2. Please note that we still use the policies (black-box) exact matching condition. As before, this is because we only have to check whether the ServiceTemplate's policies are applicable to the considered NodeType.

\title{
Matching of requirements
}

Consider the UnmatchedRequirementSet obtained when the black-box matching procedures fail. How can we treat such set? It is worth pointing that there is no need to search such requirements inside $S T$. In fact, if the ServiceTemplate ST exposes less requirements than the NodeType N, then the requirement plug-in matching condition will be satisfied. This explains why, in Definition 4.1, we still employ the

\section{N.RequirementDefinitions $\subseteq_{R} S T$. Requirements}

condition.

Observation 4.3. As we will see, (since we employ the requirement plugin matching condition) the adaptation of the white-box matched Service Template $S T$ will generate a new ServiceTemplate $S T^{\prime \prime}$ such that

$$
N \subseteq S T^{\prime \prime}
$$


Chapter 4. NodeType white-box matching (and adaptation)

\section{Matching of capabilities}

Consider a ServiceTemplate ST and a NodeType N. To understand what

\section{N.CapabilityDefinitions $\cong_{C}$ ST.Capabilities}

means we have to think about when this kind of matching occurs. The white-box approach will be used when the black-box flexible one fails. This means that $N$ exhibits some capabilities that are not visible in $S T$.

What can we do to overcome the above exposed problem? We have to search inside $S T$ the desired features. In particular, we have to look at in-nested NodeTemplate elements. To do it we will use the notation

$$
S T \rightarrow \text { NodeTemplate }
$$

to indicate that we are navigating ${ }^{2}$ the XML TOSCA Definitions tree in order to reach in-nested NodeTemplate elements.

Definition 4.2. Let $N$ be a NodeType and $S T$ be a ServiceTemplate. We say that

$$
\text { N.CapabilityDefinitions } \cong_{C} \text { ST.Capabilities }
$$

if and only if:

$\forall$ CapabilityDefinition $x \in N$.CapabilityDefinitions

$(\exists$ Capability $y \in S T$.Capabilities:

$y$. ref.type $=x$.capability Type $\vee$ y.ref.type $\vdash x$. capabilityType $)$

$\vee$

$(\exists$ Capability $y \in S T \rightarrow$ NodeTemplate.Capabilities: $y . r e f . t y p e=x$. capability Type $\vee$ y.ref.type $\vdash x$.capability Type $)$.

\footnotetext{
${ }^{2}$ The " $\rightarrow$ " notation's meaning is analogue to that of the "//" (XPath [36]) operator
} 
Chapter 4. NodeType white-box matching (and adaptation)

\section{Matching of properties}

What we want to do now is to clarify the meaning of the following condition:

$$
\text { N.PropertiesDefinition } \cong_{P R} \text { ST.Properties }
$$

As before, we have to look inside $S T$ to find the desired (unmatched) properties. To understand which in-nested elements should be considered in properties white-box matching we have to answer to the following question: by which kind of TOSCA elements could properties be exhibited? The answer is: Relationship Template and NodeTemplate.

Definition 4.3. Let $N$ be a NodeType and $S T$ be a ServiceTemplate. We say that

$$
\text { N.PropertiesDefinition } \cong_{P R} \text { ST.Properties }
$$

if and only if:

$\forall$ propertyDefinition $x \in N$.PropertiesDefinition

( $\exists$ property $y \in S T$.Properties:

the XML type of $y$ extends the one defined by $\mathrm{x}$ $\wedge$

y.name $\vdash \dashv x$. name)

$\mathrm{V}$

( $\exists$ property $y \in S T \rightarrow$ NodeTemplate.Properties:

the XML type of $y$ extends the one defined by $\mathrm{x}$

$\wedge$

y.name $\vdash \dashv x$.name)

$\vee$

( $\exists$ property $y \in S T \rightarrow$ Relationship Template.Properties:

the XML type of $y$ extends the one defined by $\mathrm{x}$ 


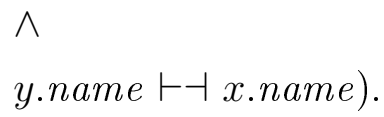

\section{Matching of interface operations}

So far, to check whether a NodeType $N$ white-box matches a ServiceTemplate $S T$, we only search for (black-box) missing features inside $S T$. If we use the same approach to explain the meaning of:

$$
N \text {.Interfaces } \cong_{I} \text { ST.Interfaces, }
$$

then we do not consider its whole meaning; indeed, to overcome the missing interface operation problem, we should also check whether combining (some of) the available operations is possible to obtain the desired one.

Definition 4.4. Let $N$ be a NodeType, ST be a ServiceTemplate and $O C_{S T}$ the set of all possible plans combining $S T$ 's operations. We say that

$$
N . \text { Interfaces } \cong_{I} \text { ST.Interfaces }
$$

if and only if:

$$
\begin{aligned}
& \forall \text { Operation } x \in N . \text { Interfaces.Interface } \\
& \quad \exists \text { Operation } y \in S T \text {.Interfaces.Interface: } \\
& \quad x \cong_{O} y \\
& \vee \\
& \exists \text { Plan } p \in O C_{S T}: \\
& \quad x \cong_{O} \text { (Operation) } p .
\end{aligned}
$$


Observation 4.4. Please note that:

- Operation elements are compared by the black-box flexible matching operator $\left(\cong_{O}\right)$. This is because we can look at those TOSCA elements only from a black-box viewpoint (since they are always shown in terms of their name and their input/output parameters);

- since the operator $\cong_{O}$ checks whether two operations expose equivalent input/output parameters, it can be used also to check if an $O p$ eration flexibly matches a Plan (provided that the latter is treated as an Operation).

\section{Concluding remark}

Looking at the step-wise definition of the white-box matching operator $\cong$ we can intuitively derive the following implication. Let $N$ be a NodeType and ST be a ServiceTemplate

$$
N \cong S T \Longrightarrow N \cong S T \text {. }
$$

This is because the white-box matching definition looks for feature both on ST.BoundaryDefinitions and inside ST. So, if all required (equivalent) features are on the boundaries of $S T$, then both flexible and whitebox matching condition are satisfied.

\subsubsection{Adaptation}

In the previous subsection we have introduced a way to white-box match a NodeType $N$ and a ServiceTemplate $S T$. Clearly, if $S T \cong N$, this does not mean that the former can be (immediately) used as a substitute for the latter. Indeed, we have to adapt $S T$ in order to be used in place of $N$. 
Chapter 4. NodeType white-box matching (and adaptation)

\section{Adaptation of capabilities and properties}

If we omit interface operations from consideration, then the adaptation process simply consist in modifying the $S T$ boundaries in order to exhibit those features that cannot be seen from a black-box viewpoint.

\section{Adaptation of interface operations}

The above adaptation approach is not enough if used to exhibit missing operations. This is because, despite it can be used to exhibit operations simply hidden inside $S T$, it cannot solve the problem of operations taken from $O C_{S T}$ (viz., the set of all possible plans combining $S T$ 's operations). Indeed, to solve the latter problem we have to:

1. look at (all) possible plans combining $S T$ 's operations and check whether some of them flexibly match the unavailable operations;

2. if this is the case, store those plans inside $S T$ and modify its boundaries in order to exhibit those plans as available operations.

\section{Full adaptation}

Once the white-box matching is done and the available ServiceTemplate $(S T)$ boundaries have been modified, we can look at $S T$ as a (new) ServiceTemplate $S T^{\prime \prime}$. Since the objective of white-box matching is to modify $S T$ in order to black-box match NodeType $N$, the condition

$$
S T^{\prime \prime} \cong N
$$

holds. It follows that we can simply reuse the (extended) oblivion boundaries adaptation approach in order to let $S T^{\prime \prime}$ be included in the adapted service $S T^{\prime}$ (which is the one with the client interacts). 


\section{Concluding remark}

So far we have implicitly assumed to have $O C_{S T}$ (the set of all possible plans combining $S T$ 's operations) to be somehow available.

In the following section we will focus on how to:

- generate (all) possible plans combining ST's operations, and

- check whether some of them flexibly match the missing operations.

\subsection{Generating plans}

In this section we will provide a solution that overcomes the plan generation problem. This solution will follows the two steps approach proposed in [5] by Brogi et al.

Functional dependency synthesis We know that each operation is equipped with functional information (i.e., its input and output parameters). This functional information defines functional dependencies within and among operations. Hence, we need to represent the relationships which state "which set of input parameters an operation requires in order to produce a set of output parameters" (intra-operation dependencies), as well as "which set of output parameters produced by an operation is required as input by another one" (inter-operation dependencies).

Furthermore, in both Sections 3.3 and 4.2 , we made our checking using some kind of semantics ${ }^{3}$. Hence, it is also necessary to represent those relationships that state "which parameters are semantically equivalent".

\footnotetext{
${ }^{3}$ We used ontologies to describe (and compare) concepts by which our features are annotated.
} 
So, we have to collect all those dependencies into a suitable data structure. As we will see (Subsection 4.3.1), the first step builds a dependency hypergraph, whose nodes represent functional attributes of operations (i.e., their input/output parameters), and whose hyperedges represent relationships among them.

Operation sequence detection Once the dependency hypergraph is built, we can proceed with the second step. Given a missing operation $O$, we can explore the hypergraph to detect which operation sequence:

- takes as input $O$ 's input parameters, and

- produces as output its output parameters.

More precisely, the parameters taken as input and produced as output by the detected sequence are semantically equivalent to those of $O$.

\subsubsection{Functional dependency synthesis}

Before describing the dependency hypergraph and how to build it, we include hereafter the definitions of hypergraph, directed hypergraph and directed hyperedge (as described in [15]).

Definition 4.5. A hypergraph is a pair $H=\langle V, E\rangle$, where

- $V=\left\{v_{1}, v_{2}, \ldots, v_{n}\right\}$ is a set of vertices (or nodes), and

- $E=\left\{E_{1}, E_{2}, \ldots, E_{m}\right\}$, with $E_{i} \subseteq V$ for $i=1, \ldots, m$, is a set of hyperedges.

Note that when $\left|E_{i}\right|=2, i=1, \ldots, m$, the hypergraph is a standard graph. 
Definition 4.6. A directed hypergraph is a hypergraph with directed hyperedges. A directed hyperedge is an ordered pair, $E=\langle X, Y\rangle$, of (possibly empty) subsets of vertices. $\mathrm{X}$ is the tail of $E$, denoted by $T(E)$, while $Y$ is its head, denoted by $H(E)$.

Figure 4.3 is illustrates an example of directed hyperedge with tail $\left\{x_{1}, x_{2}\right\}$ and head $\left\{y_{1}, y_{2}, y_{3}, y_{4}\right\}$.

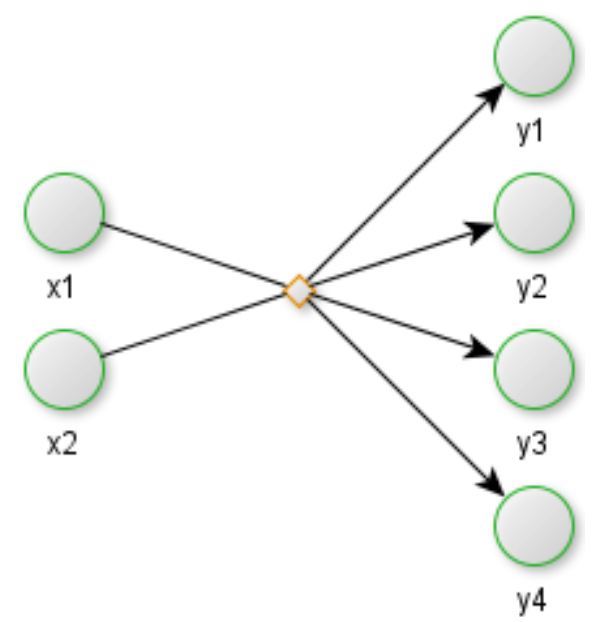

Figure 4.3: A directed hyperedge.

\section{The dependency hypergraph}

A hypergraph is a suitable notation to represent parameters (as nodes) and dependencies among them (as hyperedges). As mentioned earlier, we have to distinguish between intra-operation and inter-operation dependencies.

Definition 4.7. A labelled directed hypergraph $(E, V, l)$ is a directed hypergraph $\langle E, V\rangle$ with a labelling function $l: E \rightarrow A$ assigning to each hyperedge a label from a given alphabet $A$. 
A labelled directed hyperedge is denoted by a triple $E=\langle X, Y, a\rangle$, where $X, Y, a$ denote the tail, the head and the label of $E$, respectively.

The hyperedge labelling availability let us obtain the desired discrimination.

Intra-operation dependency Let $O$ be an Operation. The output parameters of $O$ intra-operation depend on its input parameters. It follows that there must exists a $\left(E_{\text {intra }}\right)$ hyperedge from O.InputParameters to $O$.OutputParameter labelled with $O$ :

$$
\langle\text { O.InputParameters, O.OutputParameters, } O\rangle \in E_{\text {intra }} .
$$

Inter-operation dependency To understand this kind of dependency we have to look at parameter semantics. Let $p_{1}$ be an output parameter of an operation $O_{1}$ and $p_{2}$ be an input parameter of an operation $\mathrm{O}_{2}$. It is worth noting that, if $p_{1}$.name is a subconcept of $p_{2}$.name (and, obviously, $p_{1}$.type is - the same type a or - a subtype of $p_{2}$.type), then the output parameter $p_{1}$ can be used as input to operation $\mathrm{O}_{2}$. The same substitution can be done if those parameter names are semantically equivalent.

Let us consider (separately) the above stated situations:

- let $p$ be a parameter and let $O S$ be the set of parameters whose names are (direct) sub-concept of p.name and whose types are the same type as or a subtype of $p$.type. Then there must exist a $\left(E_{\text {sub }}\right)$ hyperedge from $O S$ to $p$ :

$$
\langle O S,\{p\}, n i l\rangle \in E_{\text {sub }}
$$

- let $p_{1}$ and $p_{2}$ be two (type-compatible) parameters whose names are semantically equivalent. Since we are working on the same 
Chapter 4. NodeType white-box matching (and adaptation)

cloud service application, we can state that the problem of checking whether two operation names are semantically equivalent is not a cross-ontology one. It follows that, if two parameters have names which are semantically equivalent, then they will expose the same name. In other words, they will correspond to the same node in our hypergraph. So, there is no need of putting an hyperedge between them.

Before continuing our discussion, we have to clarify the sub-concept of relationship. With respect to the hierarchical structure of an ontology, $c$ is a (direct) sub-concept of $d$ if $c$ is a child of $d$. So, $E_{s u b}$ hyperedges will (only) be between (type-compatible) parameters which are in this kind of relationship.

Please note that if $c$ is a (direct) sub-concept of $e$ and $e$ is a (direct) sub-concept of $d$, then also $c$ is a sub-concept of $d$. So, we have to extend our sub-concept notion in terms of the dependency hypergraph. Namely, $c$ is a sub-concept of $d$ if and only if there exist a path from $c$ to $d$ which consists of sub-concept relationships (i.e., it goes through $E_{\text {sub }}$ hyperedges).

\section{Dependency hypergraph construction}

We have seen what dependency hypergraph means. The next question is how to build a dependency hypergraph.

The answer stays in the more obvious solution. Starting from the empty hypergraph $H=\langle V, E, l\rangle$ (with $V=\varnothing$ and $E=\varnothing$ ), we have to proceed for each operation $O$ as follows:

1. for each input parameter in $\in$ O.InputParameters, if in $\notin V$ then

(a) add in to $V$;

(b) modify $E$ in order to make in point to the type-compatible 
Chapter 4. NodeType white-box matching (and adaptation)

parameter $p$ (if present) such that in.name is a (direct) subconcept of $p$.name;

(c) let $S \subseteq V$ be the set of type-compatible parameters whose names are (direct) sub-concept of in.name. Add the

$$
\langle S,\{i n\}, n i l\rangle \in E_{\text {sub }}
$$

hyperedge to $E$.

2. for each output parameter out $\in$ O.OutputParameters, if out $\notin V$ then proceed as for input parameters.

3. add to $E$ the hyperedge

$$
\langle\text { O.InputParameters, O.OutputParameters, } O\rangle \in E_{\text {intra }} \text {. }
$$

Please note that there is no need to consider Plans (as operations) in this procedure. This is because Plans are in turn operation sequences. So, they will be generated again with the operation sequence detection phase 4 .

\section{Practical considerations about complexity}

While building a dependency hypergraph has exponential complexity (all pairs of operation must be composed), since the set of service components available operations is a static one, this procedure is executed only once. So, the high complexity could be paid only at the start of our (white-box) matching procedure.

Furthermore, a clever service provider could think about storing each ServiceTemplate along with its own dependency hypergraph. If this is the case, the matching cost will be significantly reduced because the

\footnotetext{
${ }^{4}$ Furthermore, if present, plans are exhibited out of service boundaries (as available operations). So, since they were matched from a black-box viewpoint, there is no need to search them from a white-box one.
} 
hypergraph will be already available (and it won't be required to build it).

Last (but not least), the above consideration suggest us to have the dependency hypergraph somewhere available when we are going to start the operation sequence detection phase. So, in the following subsection we will explain how to perform that phase assuming the dependency hypergraph availability (as input).

\subsubsection{Operation sequence detection}

We are facing the problem of white-box matching between a NodeType $N$ and a ServiceTemplate ST. More precisely, we are checking whether a $N$ 's Operation $O$ can be substituted by a sequence of operations inside $S T$ (since none of the available exposed operations flexibly matches with $O$ ). So, we have to search in the (available) dependency hypergraph for a functionally equivalent operation sequence. Before formalizing this equivalence notion, we will introduce a sub-concept (and type-compatibility) operator to increase readability.

Definition 4.8. Let $a$ and $b$ be two Operation parameters. We will say that $a$ is (type-compatible with and) sub-concept of $b(a \triangleleft b)$ if and only if

a.type is (the same as or) a sub-type of b.type $\wedge$ a.name is a sub-concept of b.name.

Definition 4.9. Let $O$ be an Operation and $O S$ be a set of Operation elements. Then $O$ is functionally equivalent to $O S(O \Leftarrow O S)$ if and only if 


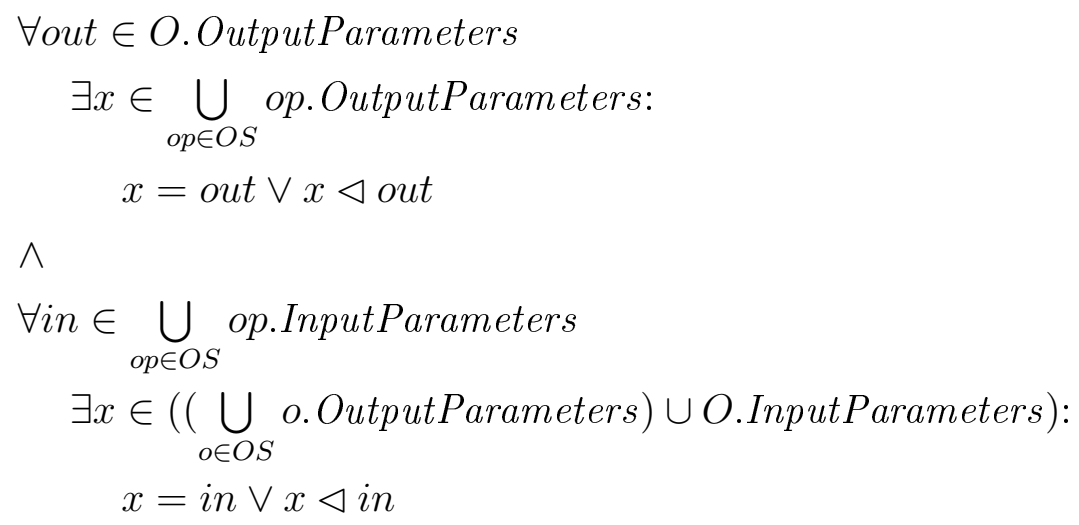

Namely, we say that a set $O S$ of Operation elements is functionally equivalent to an Operation $O$ if and only if:

- for each O's output parameter out there is a (type-compatible) output parameter $x$ which is produced by some service in $O S$ and whose name is (equivalent to or) a sub-concept of out.name, and

- for each Operation in $O S$, its input parameter names (are equivalent to or) subsume the names of (type-compatible) parameters that are given in input to $O$ or that are produced as output by some service in $O S$.

Before describing how to determine the set(s) $O S$ we have to make an observation. Why are we talking about sets (and not sequences) of Operation elements? This is because the solution we are going to introduce will discover all the possible (minimal) sets of Operation elements which satisfy the functional equivalence condition. Once those sets are available, obtaining the desired sequence is immediate. Indeed, we only have to start from $O$ 's input parameters and add each Operation in $O S$ following the hyperedges of the dependency hypergraph. 
So, in the following paragraphs we will show how to discover the minimal sets of Operation elements 5 .

\section{Discovering sets of Operation elements}

As one may expect, the set discovering consists of a visit of the dependency hypergraph. More precisely, OperationSEtsDiscovering (Figure 4.4) visits the dependency hypergraph starting from those vertices corresponding to the parameters (equivalent ${ }^{6}$ to those) outputted by $O$, and it goes on by exploring backwards the hyperedges until reaching (if possible) the input parameters that $O$ requires. As we will see, OperATIONSETSDisCOVERING detects all the minimal sets $O S$ of Operation elements such that $O \Leftarrow O S$.

OPERATIONSETSDISCOVERING requires five input parameters: the dependency hypergraph $H$, the Operation to "discover" $O$, the set composition of the operations selected so far (initially empty), the set of the needed output parameters to be generated (initially the O's outputs), and the set of the available output parameters (initially $O$ 's inputs).

If no more output parameters need to be generated (needed $=\varnothing$ ), OPERATIONSETSDISCOVERING stores the set composition (such that $O \Leftarrow$ composition) and the algorithm is stopped (lines 1-4).

Otherwise, the algorithm employs ExTRACT $]^{7}$ to (non-deterministical-

\footnotetext{
${ }^{5}$ The algorithm and the relative results are an adaptation (to our problem) of those proposed by Corfini [11.

${ }^{6}$ Please note that, since the Operation $O$ comes from a different environment, there could be some mismatchings between the relative ontologies. For the sake of simplicity (and since - according to Assumptions 3.2 and 3.3 - we can check equivalent concepts before starting OpERATIONSETSDiscovering), in the following we do not consider the cross-ontology problem.

${ }^{7}$ It is worth noting that EXTRACT implementation strictly depends on the way in which the set needed is implemented. So, for the sake of simplicity, we assume the availability of that procedure.
} 
Require: dependency hypergraph $H$, Operation $O$, set composition, set needed, set available

1: if needed $=\varnothing$ then

2: $\quad$ store composition;

3: exit;

4: end if

5: out $=\operatorname{ExTRACT}($ needed $)$;

6: Ops $=\{o \mid \exists c \in$ o.OutputParameters $: c=$ out $\vee c \triangleleft$ out $)\}$;

7: if $O p s=\varnothing$ then

8: fail;

9: end if

10: for all Operation op $\in$ Ops do

11: $\quad$ composition' $=$ composition $\cup\{o p\}$;

12: for all Operation $p \in$ composition do

13: $\quad R=$ composition $\backslash\{p\}$;

14: $\quad$ if $\nexists x \in$ p.OutputParameters:

$\exists o \in$ O.OutputParameters :

$\left(x \triangleleft o \wedge \nexists z \in \bigcup_{r \in R}\right.$ r.OutputParameters: $\left.z \triangleleft o\right)$ $\mathrm{V}$

$\exists i \in \bigcup_{r \in R}$ r.InputParameters :

$\left(x \triangleleft i \wedge \nexists z \in \bigcup_{r \in R}\right.$ r.OutputParameters $\cup$ O.InputParameters : $z \triangleleft i)$ ) then

15: fail

16: $\quad$ end if

17: end for

18: $\quad$ available $=$ available $\cup$ op.OutputParameters;

19: $\quad$ needed' $=\{x \mid x \in$ (needed $\cup\{y \mid y \in$ op.InputParameters

$\wedge \nexists z \in$ O.InputParameters $: z \triangleleft y\})$

$\wedge \nexists a \in$ available' $: a \triangleleft x\}$;

20: OperationSetsDiscovering(H,O,composition',needed',available');

21: end for

Figure 4.4: OpERATIONSETSDiscOVERING algorithm. 
ly) withdraw an output parameter out from the set of needed outputs (line 5). Once out has been taken, OpERATIONSETSDISCOVERING proceeds in computing the set Ops of the operations which produce a type-compatible parameter whose name is a sub-concept of out.name (line 6). Afterwards, if $O p s$ is empty (i.e., out cannot be generated by any available operation), then OPERATIONSETSDISCOVERING fails (since the operation cannot be matched by any sequence of available Operation elements - lines 7-9).

Otherwise, for each operation op in Ops (line 10), the algorithm adds op to composition (line 11) and updates the sets available and needed by adding them the outputs of op (line 18) and the unavailable inputs of op (line 19), respectively. Then, a new OperationSetsDiscovering instance is started on the computed sets (line 20).

In the next paragraph, we will discuss in detail how to reject (by failing) non-minimal operation sets (lines 12-17).

\section{Minimality of discovered sets}

As already anticipated, the role of the loop at lines 12-17 is to discard (by failing) any non-minimal set $O S$ of Operation elements such that $O \Leftarrow O S$. Going on in their explanation requires to formalize the obvious notion of minimality.

Definition 4.10. Let $O$ be an Operation and $O S$ be a set of Operation elements such that $O \Leftarrow O S$. $O S$ is minimal if and only if

$$
\nexists O S^{\prime} \subset O S: O \Leftarrow O S^{\prime}
$$

Intuitively speaking, the loop under consideration checks whether the inclusion of the new Operation op in the set composition makes some other Operation elements in composition not strictly necessary to obtain $O \Leftarrow$ composition. 
Observation 4.5. It is worth noting that op is certainly needed to satisfy the condition $O \Leftarrow$ composition, since the set composition $\backslash\{o p\}$ is not able to produce out (line 6). Note, indeed, that OperationSETsDisCOVERING does not consider the operation op (line 12).

OPERATIONSETSDISCOVERING proceeds by checking, for each Operation $p$ in composition (line 12), whether the condition at line 14 holds. Such condition is true if all the output parameters produced by $p$ are already available since:

- they are generated by the other Operation elements in composition $\cup\{o p\} \backslash\{p\}$, or

- they are provided as input parameters of $O$.

In other words, if the condition at lines 14 holds, then the inclusion of $o p$ in the set of operations has made the Operation $p$ not strictly necessary to achieve the goal. If this is the case, OpERATIONSETSDiscovERING fails (line 15) to avoid constructing non-minimal sets of Operation elements.

It is worth noting that, despite the condition under consideration (line 14) is quite verbose, its practical checking consists only of a few trivial operations among small sets of data.

Last (but not least), we have to answer to the following question: are we sure that line 14 condition is both necessary and sufficient to establish the minimality of a set $O S$ of operations (such that $O \Leftarrow O S$ )?

Property 4.1. Let $O$ be an Operation and let $O S$ be a set of Operation elements such that $O \Leftarrow O S$. $O S$ is minimal if and only if:

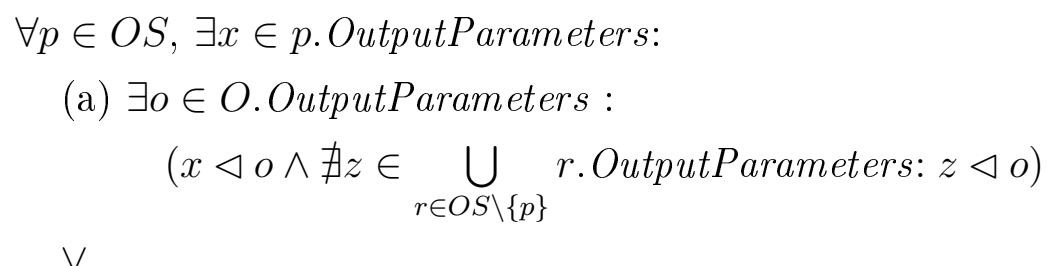


(b) $\exists i \in \bigcup_{r \in O S \backslash\{p\}} r$.InputParameters :

$$
\begin{aligned}
& (x \triangleleft i \wedge \\
& \qquad z \in \bigcup_{r \in O S \backslash\{p\}} r \text { OutputParameters } \cup \text { O.InputParameters : } \\
& \quad z \triangleleft i))
\end{aligned}
$$

Proof. We have to prove a $\Longleftrightarrow$ relationship. So, we will proceed demonstrating the two directions separately.

$(\Longrightarrow)$ Let us assume that $O S$ (such that $O \Leftarrow O S$ ) is minimal (see Definition 4.10). This means that the following condition holds.

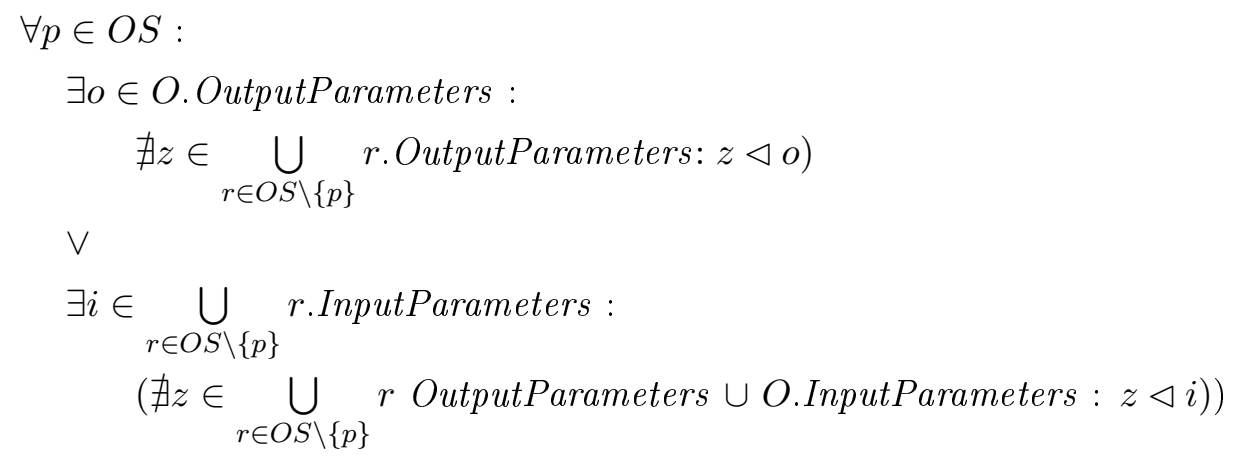

Since $O \Leftarrow O S$, applying what Definition 4.9 states, we can derive the desired thesis.

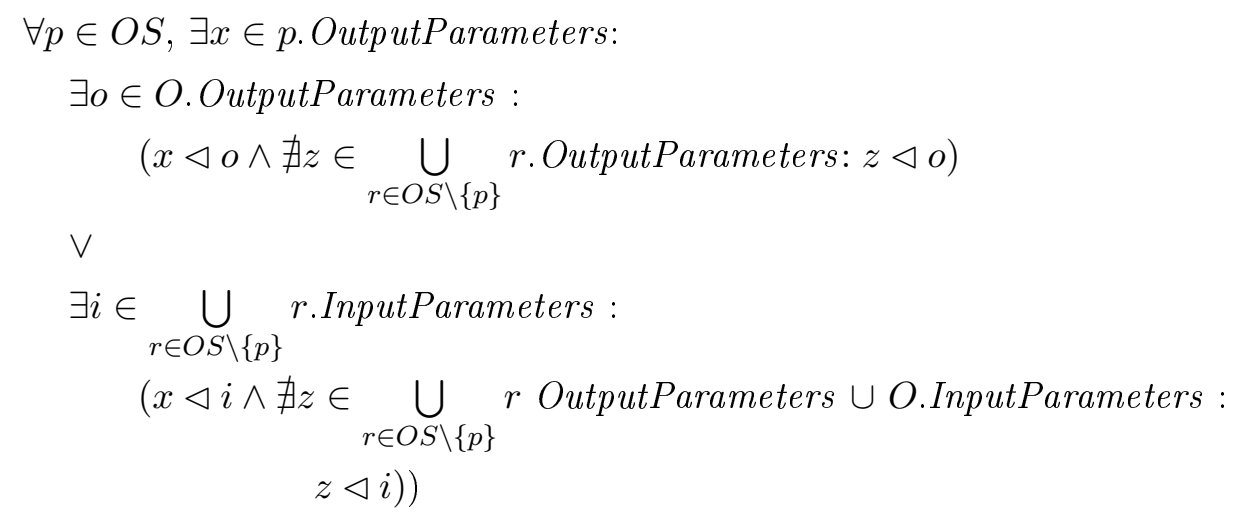

$(\Longrightarrow)$ We will prove the desired property by contradiction. Let us assume that 
$O S$ is not minimal. This means that the following condition holds.

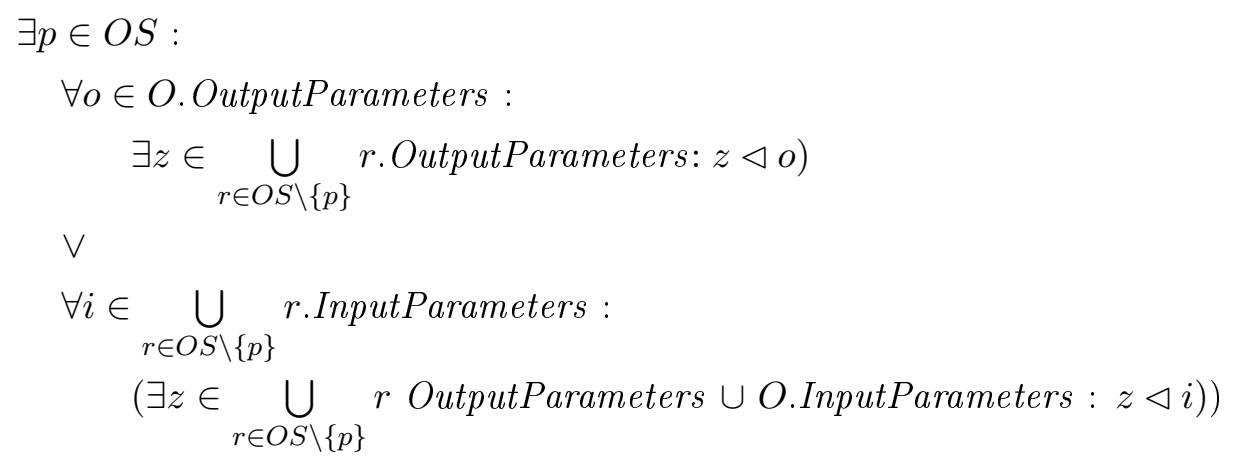

Now, since

$$
\begin{gathered}
\exists p \in O S(\forall a \in A(\exists b \in B: P(p, a, b))) \Longrightarrow \exists p \in O S(\nexists a \in A(\nexists b \in B: \\
P(p, a, b)))
\end{gathered}
$$

we obtain the following condition.

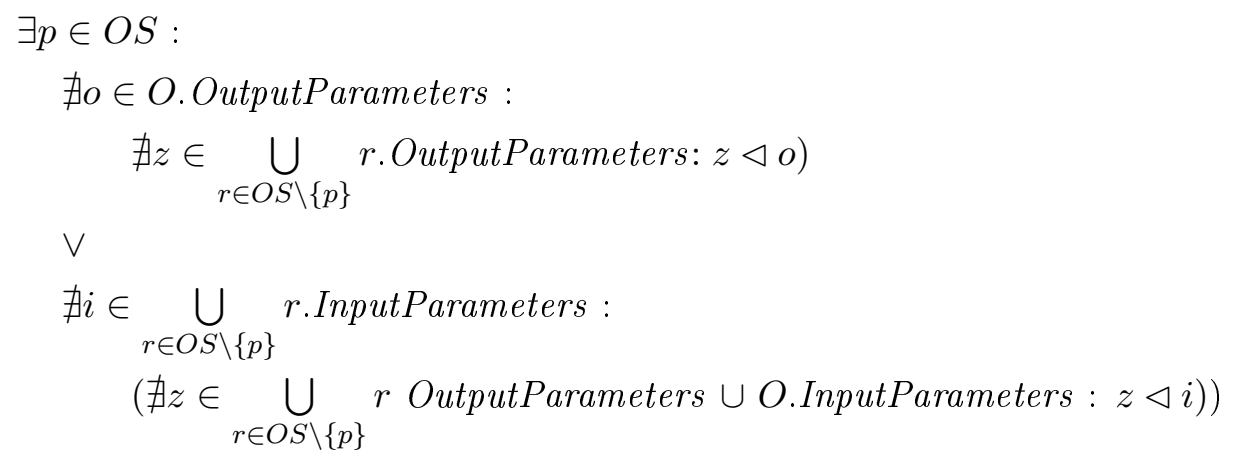

The above condition is clearly in contradiction with the (assumed) right part of the desired property. This implies that $O S$ is minimal.

\section{Soundness, completeness and complexity}

We are going to conclude this Subsection 4.3.2 with a (brief) discussion of the OpERATIONSETSDiscovering soundness, completeness and complexity. 
Soundness As mentioned before, each instance of OpERATIONSETSDISCOVERING stores a set of Operation elements which is functionally equivalent $(\Leftarrow)$ with the searched Operation $O$. The following proposition establishes the soundness of the proposed algorithm, namely, that each stored set of operations satisfies the functional equivalence condition (see Definition 4.9).

Proposition 4.1. Let $O S$ be a set of Operation elements stored by the OperationSetsDiscovering for a given Operation $O$. Then, $O \Leftarrow O S$.

Proof. The proof is organised in three steps. First, we will establish an invariant property $\Phi$ which holds for every invocation of OperATionSetsDiscovering (1). Then we will prove that $\Phi$ implies $O \Leftarrow O S$ when the algorithm terminates (2). Finally, we will demonstrate that OperationSETsDiscovering always terminates (3).

(1) Please note that the set needed (which initially contains the desired operation $O$ output parameters) is updated whenever a new Operation op is added to the composition set. More precisely, OPERATIONSETSDISCOVERING

- adds to needed the unavailable op.InputParameters, and

- removes from needed those elements with which op.OutputParameters are in the $\triangleleft$ relation.

So, whenever a recursive call of OpERATIONSETSDiscovering is performed, the following invariant property $\Phi$ holds.

$$
\begin{aligned}
& \Phi \equiv \text { needed }= \\
& \qquad \begin{aligned}
&\{x \mid x \in(\text { O.OutputParameters } \cup \\
&\left\{u \mid u \in \bigcup_{p \in O S}\right. \text { p.InputParameters } \\
&\wedge \nexists v \in \text { O.InputParameters }: v \triangleleft u\}) \\
&\left.\wedge \nexists y \in \bigcup_{p \in O S} p . \text { OutputParameters }: y \triangleleft x\right\}
\end{aligned}
\end{aligned}
$$


where $O S$ denotes the set of Operation elements selected so far (i.e., the set composition).

(2) Now, we know that OperationSETsDiscovering returns the set composition (only) when needed is empty. But, what does the condition "needed $=\varnothing$ " imply? Since $\Phi$ holds, it follows that:

$$
\begin{aligned}
& \nexists x: x \in(\text { O.OutputParameters } \cup \\
& \left\{u \mid u \in \bigcup_{p \in O S}\right. \text { p.InputParameters } \\
& \quad \wedge \nexists v \in \text { O.InputParameters }: v \triangleleft u\}) \\
& \left.\wedge \nexists y \in \bigcup_{p \in O S} \text { p.OutputParameters }: y \triangleleft x\right\}
\end{aligned}
$$

Then, applying the following logical rules:

- $\nexists x:(x \in A \cup B \wedge \nexists y \in C: P(x, y)) \Longrightarrow$

$$
\begin{aligned}
& \forall x:(x \in A \Longrightarrow \exists y \in C: P(x, y)) \wedge \\
& (x \in B \Longrightarrow \exists y \in C: P(x, y))) ;
\end{aligned}
$$

- $((A \wedge \neg B) \Longrightarrow C) \Longrightarrow(A \Longrightarrow(B \vee C))$,

we could derive the following condition.

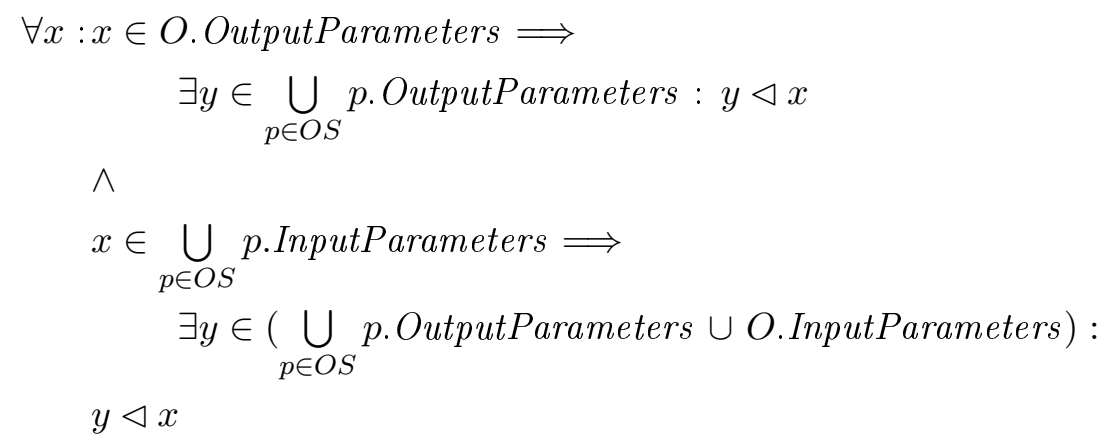

Looking at the above logical expression, we observe that (by Definition 4.9 it follows that $O \Leftarrow O S$. Hence the invariant property $\Phi$ guarantees that when needed $=\varnothing$ then composition is a set of services that satisfies the functional equivalence condition.

(3) To complete our proof, we have to show that OperationSetsDiscovering always terminates. First, let us remark that, if $O p s$ (i.e., the set of those Operation elements which produce out - or 
Chapter 4. NodeType white-box matching (and adaptation)

a parameter $c$ such that $c \triangleleft$ out) is not empty, then none of the operations in such a set is already contained in composition (since if it were contained, then out would not still belong to needed). This implies that each available Operation can be inserted in composition at most once.

Now, let candidates be the set of all Service Template operations which are candidate for insertion in composition. Since each Operation can be inserted in composition at most once, the candidates set cardinality will be decreased by one at every OpERATIONSETSDISCOVERING invocation.

Then, the last recursive call of the algorithm under consideration:

- either succeeds (if needed $=\varnothing$ ), or

- fails (because of the absence of candidate operations).

The above introduced Proposition 4.1, along with Property 4.1, let us derive the following result.

Proposition 4.2. Let $O S$ be a set of Operation elements returned by OperationSets Discovering for a given Operation $O$. Then, $O S$ is minimal and the condition $O \Leftarrow O S$ holds.

Completeness We have shown that each result set stored by OpERATIONSETSDISCOVERING satisfies the functional equivalence condition. Now we have to answer to the following question: does OPERATIONSETSDISCOVERING store all the functional equivalent minimal sets? Hereafter we will show that this is the case.

Proposition 4.3. Let $O S=\left\{O_{1}, O_{2}, \ldots, O_{n}\right\}$ be a minimal set of Operation elements such that $O \Leftarrow O S$. If there exists such a set of operations, then it will be returned by OpERATIONSETSDisCOVERING. 
Proof. Suppose by contradiction that there exists a minimal operation set $O S=\left\{O_{1}, O_{2}, \ldots, O_{n}\right\}$ such that $O \Leftarrow O S$. Suppose also that $O S$ is not stored by OperationSetsDiscovering. Is it possible? To answer this question we have to detect why $O S$ can be discarded.

(A) There exists some operation $O_{i} \in O S$ which is not selected by OpERATIONSETSDISCOVERING. In other words, none of the recursive calls of the analysed algorithm extracts from the needed set a parameter out $t_{i}$ outputted by $O_{i}$. This is possible in the following two cases:

- $O_{i}$ generates no concepts useful to match $O$. In other words, it produces neither parameters which belongs to O.OutputParameters, nor parameters taken as input by another operation in $O S$. If this is the case, $O_{i}$ is completely useless to obtain $O \Leftarrow O S$. It follows the desired contradiction: the set $O S$ is not minimal (as assumed).

- $O_{i}$ generates a parameter $c$ useful (to obtain $O \Leftarrow O S$ ) but $c$ is also produced by another Operation $O_{j} \in O S(i \neq j)$. Indeed, suppose that (at some step $n$ ) OperationSETSDiscovering extracts from the needed set a parameter out $\neq c$ which is produced by $O_{j}$. This causes the following steps.

(i) $O_{j}$ is added to composition;

(ii) the output parameters of $P_{j}$ are removed from the needed set and added to the set of available ones.

It is worth noting that also the parameter $c$ (if present) is removed from needed and added to available. So, $c$ will never be extracted from the needed set (i.e., $O_{i}$ will never be selected by OperationSetsDiscovering). Consequently, $O_{i}$ is not strictly necessary to obtain $O \Leftarrow O S$. We earned the desired contradiction: the set $O S$ is not minimal (as assumed).

(B) The set (or a subset of) $O S=\left\{O_{1}, O_{2}, \ldots, O_{n}\right\}$ is neither generated nor stored by OperationSetsDiscovering. Why could this happen? Let us consider an Operation $O_{i} \in O S$ which generates $c$ and a Operation $O_{j} \in O S\left(O_{i} \neq O_{j}\right)$ which generates out ${ }_{j}$ 
Chapter 4. NodeType white-box matching (and adaptation)

and $c$. Suppose now that (at some step $n$ ) the algorithm extracts $c$ from the needed set and selects $O_{i}$. The algorithm then goes on and when (at some step $m>n$ ) out ${ }_{j}$ is withdrawn from needed, OperationSetsDiscovering selects $O_{j}$. Consequently, $O_{i}$ becomes useless, since $c$ is also produced by $O_{j}$ (Property 4.1 does not hold). Thus, we obtain the desired contradiction (since the set of operations $O S$ is not minimal).

The above consideration let us take the desired conclusion: OperATIONSETSDisCOVERING stores all the minimal set of Operation elements which are functionally equivalent $(\Leftarrow)$ to the provided Operation $O$.

Worst-case (time) complexity Finally, we will outline a worst-case analysis of the algorithm time complexity. The analysis requires to consider a possible execution of OPERATIONSETSDiSCOVERING. So, let $O P S$ be the set of available Operation elements.

- The first instance of OpERATIONSETSDiscovering extracts

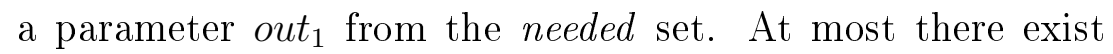
$|O P S|$ operations which output the desired parameter (or a parameter $c_{1}$ such that $\left.c_{1} \triangleleft o_{1} t_{1}\right)$. Hence, OpERATIONSETSDisCOVERING splits in $|O P S|$ instances.

- Consider now the $i$-th instance generated by first step of the algorithm (in which composition $=\left\{O_{i}\right\}$ ). Such an instance, in

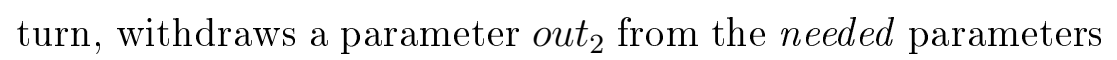
set. At most there exists $|O P S|-1$ available operations which produce the desired parameter (or a parameter $c_{2}$ such that $\left.c_{2} \triangleleft o u t_{2}\right)$. Thus, the instance under consideration splits in $|O P S|-1$ instances.

Therefore, OpERATIONSETSDISCOVERING generates

$$
|O P S| \times(|O P S|-1) \times(|O P S|-2) \times \ldots \times 2 \times 1
$$


instances.

Furthermore, each instance complexity is dominated by the minimality check. This check executes at most $O\left(|O P S|^{2}\right)$ comparisons. Then, each instance of OPERATIONSETSDiscovering costs $O\left(|O P S|^{3}\right.$ ) (since it executes the minimality check for each operation producing the out parameter extracted from the needed set).

The above consideration let us conclude that OPERATIONSETSDisCOVERING requires exponential time.

$$
T_{\text {OperationsetDiscovering }}(O P S) \in N P \text {. }
$$

\section{Practical consideration about complexity}

A fussy reader could think our solution is expensive and inefficient.

Please note that the OPERATIONSETSDISCOVERING must be executed by a service provider. In other words, it will be executed in a cloud environment and so the computational power is - potentially - infinite. This means that a clever implementation of the proposed algorithm could exploit its recursive definition to enforce parallelism.

Furthermore, (in an ideal situation) each instance of OPERATIONSETSDisCOVERING could be executed by a different concurrent activity. If this is the case, the time complexity will be decreased significantly. Indeed, it can (potentially) become polynomial in the number of available operation $O P S$.

DIGRESSION: $N P$-hardness of the considered problem

Someone could think that our solution is too expensive. So, before concluding, we want to show that the problem to be solved is in turn an expensive one. More precisely, we want to discuss the $N P$-hardness of such a problem. To do it, we will connect it with the subset sum problem (which is known to be $N P$-hard [34]). 
For the sake of simplicity let us make the following assumptions:

o the hypergraph $H$ is such that $\forall i:\left|E_{i}\right|=2$ (i.e., $H$ is a graph);

o we do not matter about semantics (i.e., we want to find only parameters which have the same type and the same name).

The above assumptions simplify quite much our problem. Now we are considering the situation of finding a sub-graph of the (hyper-)graph $H$ such that its borders are composed by those vertices corresponding to the input/output parameters of $O$.

Let us now simplify more our problem: we only want to determine the above mentioned borders (i.e., the sets of nodes corresponding to the input/output parameters of $O$ ). So, (since we are not worrying about interconnections) we are restricting our search to only the set $V$ of vertices.

Let us now label each node with a different integer number. It follows that the whole set of $O$ input/output parameters could be seen as an integer value sum (which is the sum of integer values assigned to each parameter in the $O$ input/output parameters set).

So, our problem now consists of finding (one of) the subsets of $V$ whose value sum is equal to sum. This clearly correspond to the subset sum problem.

Therefore, simplifying the problem under consideration we can connect it with a well known $N P$-hard problem. This means that the considered problem (of detecting a part of the dependency hypergraph such that required) is in turn $N P$-hard. 
Chapter 4. NodeType white-box matching (and adaptation)

\subsubsection{Example}

The objective of this subsection is to show how the plan generation problem could be solved. We will proceed as follows: we will start by giving the set of available operation and showing the relative dependency hypergraph; then, we will introduce the target operation (i.e., the operation which requires the plan generation - if possible - to be matched); finally, we will perform a possible execution of the OPERATIONSETSDISCOVERING algorithm.

\section{The dependency hypergraph}

Suppose the availability of a ServiceTemplate such that in Figure 4.1 . Furthermore, suppose that the WeatherApp's GetWeather interface exhibits the operations reported in the following table.

\begin{tabular}{|l|l|l|}
\hline Name & $\begin{array}{l}\text { Input } \\
\text { parameters }\end{array}$ & $\begin{array}{l}\text { Output } \\
\text { parameters }\end{array}$ \\
\hline weatherInfo & $\begin{array}{l}\{\text { country, } \\
\text { city }\}\end{array}$ & $\begin{array}{l}\text { weather, } \\
\text { umidity, } \\
\text { windSpeed, } \\
\text { temperature }\}\end{array}$ \\
\hline getGMT & $\varnothing$ & $\{$ gmt $\}$ \\
\hline getTemperature & $\begin{array}{l}\{\text { country, } \\
\text { city }\}\end{array}$ & temperature $\}$ \\
\hline perceivedTemperature & $\begin{array}{l}\{\text { windPower, } \\
\text { umidity, } \\
\text { temperature }\}\end{array}$ & \{perceived $\}$ \\
\hline
\end{tabular}

The above given table, along with the ontology of Figure 4.5, let us build the desired hypergraph. According to what stated at the end of Subsection 4.3.1, we assume the availability of the desired dependency hypergraph (see Figure 4.6). 


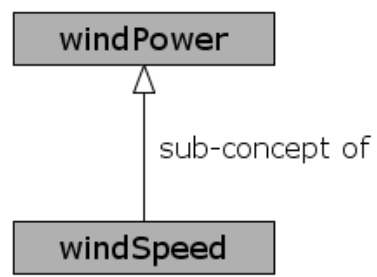

Figure 4.5: Parameters ontology example.

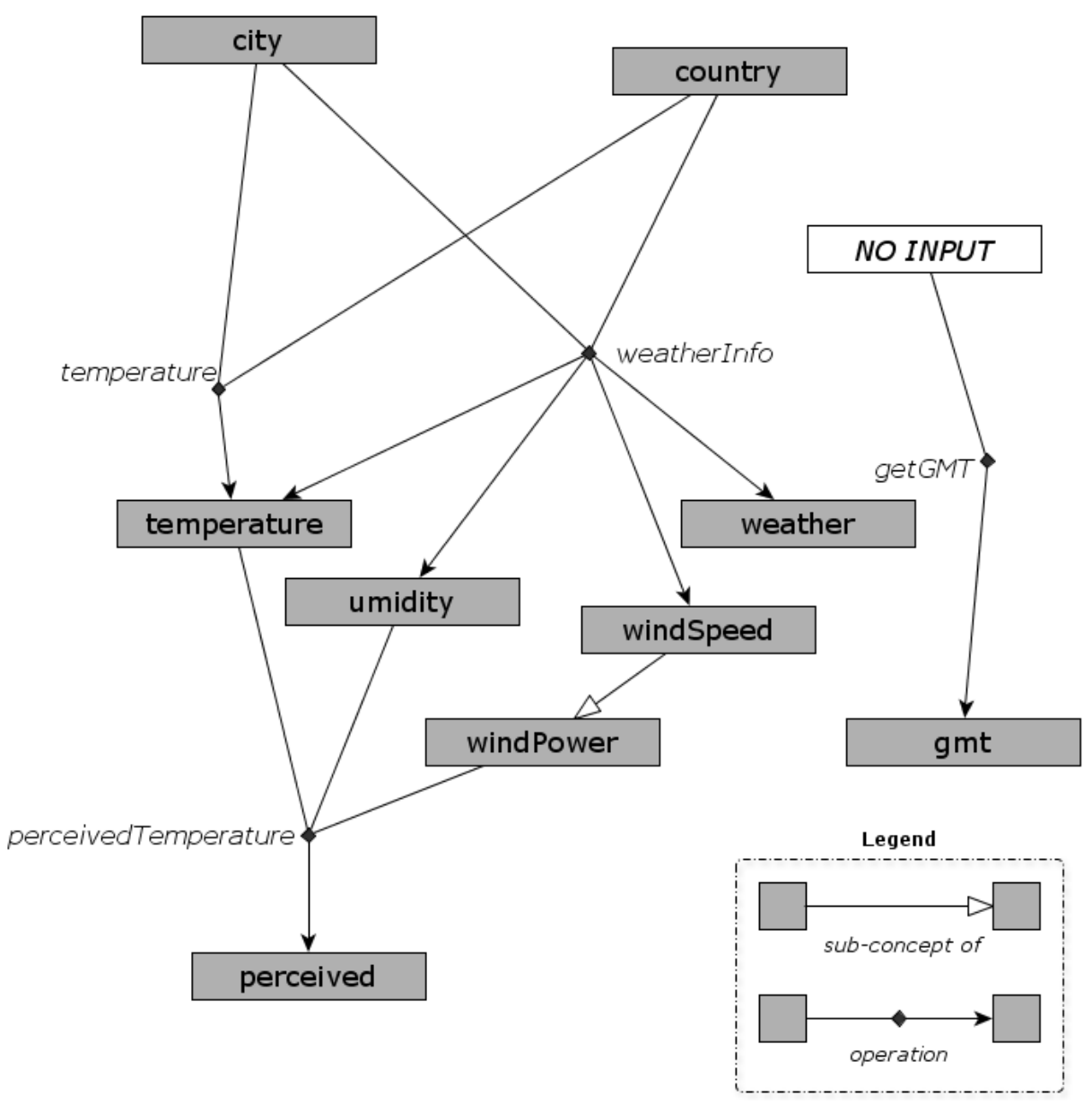

Figure 4.6: Dependency hypergraph example. 
Chapter 4. NodeType white-box matching (and adaptation)

\section{The target Operation}

Consider the situation in which our matching procedure needs the Operation getPerceivedTemperatureAndTime which:

- takes $\{$ state, city $\}$ as input parameters set;

- outputs the \{greenwichMeanTime, temperature, perceived $\}$ set of parameters.

In the following we will refer to the desired operation as $O$.

Suppose now the unavailability of $O$ in the ServiceTemplate under consideration. This means that we have to check whether the condition

$$
\exists \text { Plan } p \in O C_{S T}: x \cong_{O}(\text { Operation }) p .
$$

is satisfied. To do it, we need to solve the plan generation problem.

\section{OPERATIONSETSDISCOVERING simulation}

We start observing that some $O$ parameters are not findable in those available with the Service Template under consideration. This is the case of the "state" input parameter and the "greenwichMeanTime" output parameter. It is worth nothing that those parameters could be matched ${ }^{8}$ with the available "country" and "gmt" parameter. So, for the sake of simplicity, in the following we will consider an Operation $O^{\prime}$ (which exposes the semantically equivalent available parameters) instead of the real Operation $O$.

Now, let us start the OperationSETsDiscovering algorithm. Its first instance receives the following input (in addition to the dependency hypergraph $H$ and the target operation $O^{\prime}$ ):

\footnotetext{
${ }^{8}$ With a cross-ontology matching analogue to that exposed in Sections 3.3 and 4.2
} 


$$
\begin{gathered}
\text { composition }=\varnothing \\
\text { needed }=\{\text { gmt, temperature, perceived }\} \\
\text { available }=\varnothing
\end{gathered}
$$

Clearly, the condition needed $=\varnothing$ is not satisfied. Therefore, the algorithm proceeds in withdrawing one of the needed outputs: "gmt", for example. This means that the set Ops available operations (whose output is equivalent to - or compatible with - "gmt") is composed only by the getGMT operation. So, OperationSETsDiscovering adds the first Operation to composition and updates the needed and available sets (removing "gmt" and adding "gmt", respectively). Once these operations have been performed, the discovering procedure goes on with a new instance.

The second instance of OPERATIONSETSDiscovering receives the following input (along with the hypergraph $H$ and the Operation $O^{\prime}$ ):

$$
\begin{gathered}
\text { composition }=\{\text { get } G M T\} \\
\text { needed }=\{\text { temperature }, \text { perceived }\} \\
\text { available }=\{\mathrm{gmt}\}
\end{gathered}
$$

As before, the set of needed output parameters is not empty. So, the algorithm can withdraw another parameter from such set: suppose (for example) that the parameter "perceived" is taken. As it was for the previous instance, there is only one operation that generates an equivalent or compatible parameter: perceivedTemperature. Therefore that Operation is added to the composition set. Differently from before, (before adding the new operation) the composition set is not empty. Therefore, it must be runt the usefulness check on that operation (getGMT) which it is contained in composition. Obviously, this usefulness condition is not satisfied. So, the procedure can go on updating the needed and available sets. More precisely: 
- "perceived" is removed from the needed set and the unavailable input parameters of perceivedTemperature are added to the same set;

- "perceived" is added to the available parameters set.

Then, a new instance with the computed sets is started.

The third instance of the algorithm under consideration receives the following input (in addition to $H$ - the dependency hypergraph - and $O^{\prime}$ - the target operation):

$$
\begin{gathered}
\text { composition }=\{\text { getGMT, perceivedTemperature }\} \\
\text { needed }=\{\text { temperature }, \text { windPower }, \text { umidity }\} \\
\text { available }=\{\text { gmt }, \text { perceived }\}
\end{gathered}
$$

Since needed $\neq \varnothing$, OperationSETSDiscovering withdraws a parameter from that set. Suppose the extracted parameter is "temperature". Differently from before, two different operations generate the desired parameter. So, we need to consider them both.

weatherInfo This operation is added to composition and the usefulness of the already contained operations is checked. Since all of them are useful, the execution proceeds with the usual behaviour: needed and available are updated $9^{9}$ and a new instance $A$ (whose input is composed by the computed sets) is runt.

getTemperature Similarly as before, the sets are updated and a new instance $B$ is executed.

\footnotetext{
${ }^{9}$ It is worth noting what happens to the "windPower" needed parameter. Since weatherInfo generates a parameter "windSpeed" which is in the sub-concept hypergraph relation, it is removed from the needed set (and "windSpeed" is added to the available one).
} 
Consider the instance $A$. Its input (in addition to the dependency hypergraph $H$ and the target Operation $O^{\prime}$ ) consists of:

$$
\begin{gathered}
\text { composition }=\{\text { getGMT, perceivedTemperature, weatherInfo }\} \\
\text { needed }=\varnothing \\
\text { available }=\{\text { gmt, perceived, weather, windSpeed, umidity, } \\
\text { temperature }\}
\end{gathered}
$$

Differently from previous instances, needed $=\varnothing$. Therefore, the set composition is stored (since it is capable to exhibit - more than - the required input/output parameter) and the $A$ instance is stopped.

Consider now the instance $B$. Its input consist of (the dependency hypergraph $H$, the operation $O^{\prime}$ and):

$$
\begin{gathered}
\text { composition }=\{\text { getGMT, perceivedTemperature, getTemperature }\} \\
\text { needed }=\{\text { windPower }, \text { umidity }\} \\
\text { available }=\{\text { gmt }, \text { perceived, temperature }\}
\end{gathered}
$$

As already happened, needed $\neq \varnothing$. This means that another parameter ("windPower", for example) is withdrawn from that set. Then, the set Ops is computed and, since it contains only perceivedTemperature, that Operation is added to composition. Once this has been done, the minimality checking is performed: is there any operation which makes the composition set not minimal? Please note that the only getTemperature output parameter is produced also by the weatherInfo operation. So, since the minimality condition is not satisfied, the execution is stopped by failing. 
Chapter 4. NodeType white-box matching (and adaptation)

\section{Plan generation}

The (possible) execution shown in the previous paragraph has produced the storage of the following set.

composition $=\{$ getGMT, perceivedTemperature, weatherInfo $\}$

We are going to show how to exploit this set in plan generation.

The procedure simply consist of answering iteratively to the following question: which operations in composition are performable? Let us start with the target operation input parameters. With this set of parameters we can execute the weatherInfo and $\operatorname{get} G M T T^{10}$ operations. Then, the available parameters set is updated with those produced by the performed operations. Once this has been done, also perceivedTemperature is performable.

With the above (simple) procedure we can generate the plan of Figure 4.7.

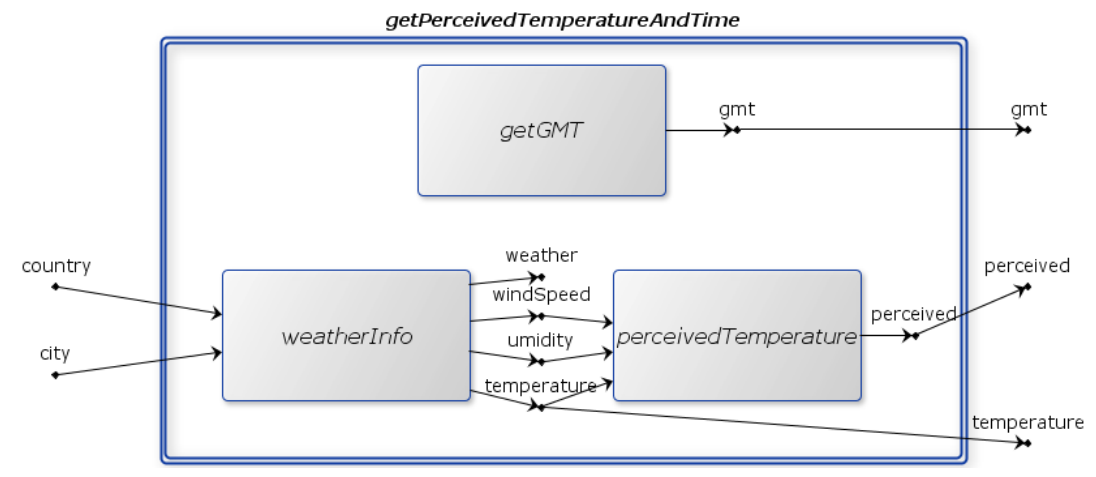

Figure 4.7: Example of generated plan.

${ }^{10}$ This operation does not require any input parameter. 
Chapter 4. NodeType white-box matching (and adaptation)

\subsection{Concluding remarks}

This chapter has provided a solution to the white-box matching problem. This solution completes the discussion about the type-checking (and adaptation) between a NodeType $N$ and a ServiceTemplate ST. Before going on, it is worth to make some final observations.

\subsubsection{A complete example}

Consider the problem of matching between the ServiceTemplate ST and the NodeType WeatherAppType (see Figure 4.8). Suppose that the GetWeather interfaces (inside and on the boundaries of $S T$ ) exhibit the same operation as those of the example in Subsection 4.3.3. Furthermore, suppose that the getPerceivedTemperatureAndTime interface (of Weather AppType) declares to export the homonym operation (which is the same as the target operation of the example in Subsection 4.3.3.

If we black-box match the ServiceTemplate and the NodeType under consideration, then we obtain a fail (since $S T$ does not expose the Name property and the desired operation). In other words, accordingly to Figure 3.10, we obtain:

$$
\begin{gathered}
\text { unmatchedRequirementSet }=\varnothing \\
\text { unmatchedCapabilitySet }=\varnothing \\
\text { unmatchedOperationSet }=\{\text { getPerceivedTemperatureAndTime }\} \\
\text { unmatchedPropertySet }=\{\text { Name }\}
\end{gathered}
$$

So, we have to proceed with the white-box viewpoint approach explained in this chapter.

First, we have to search for the Name property in the nodes inside $S T$. Our property search, as mentioned in (the relative paragraph of) 

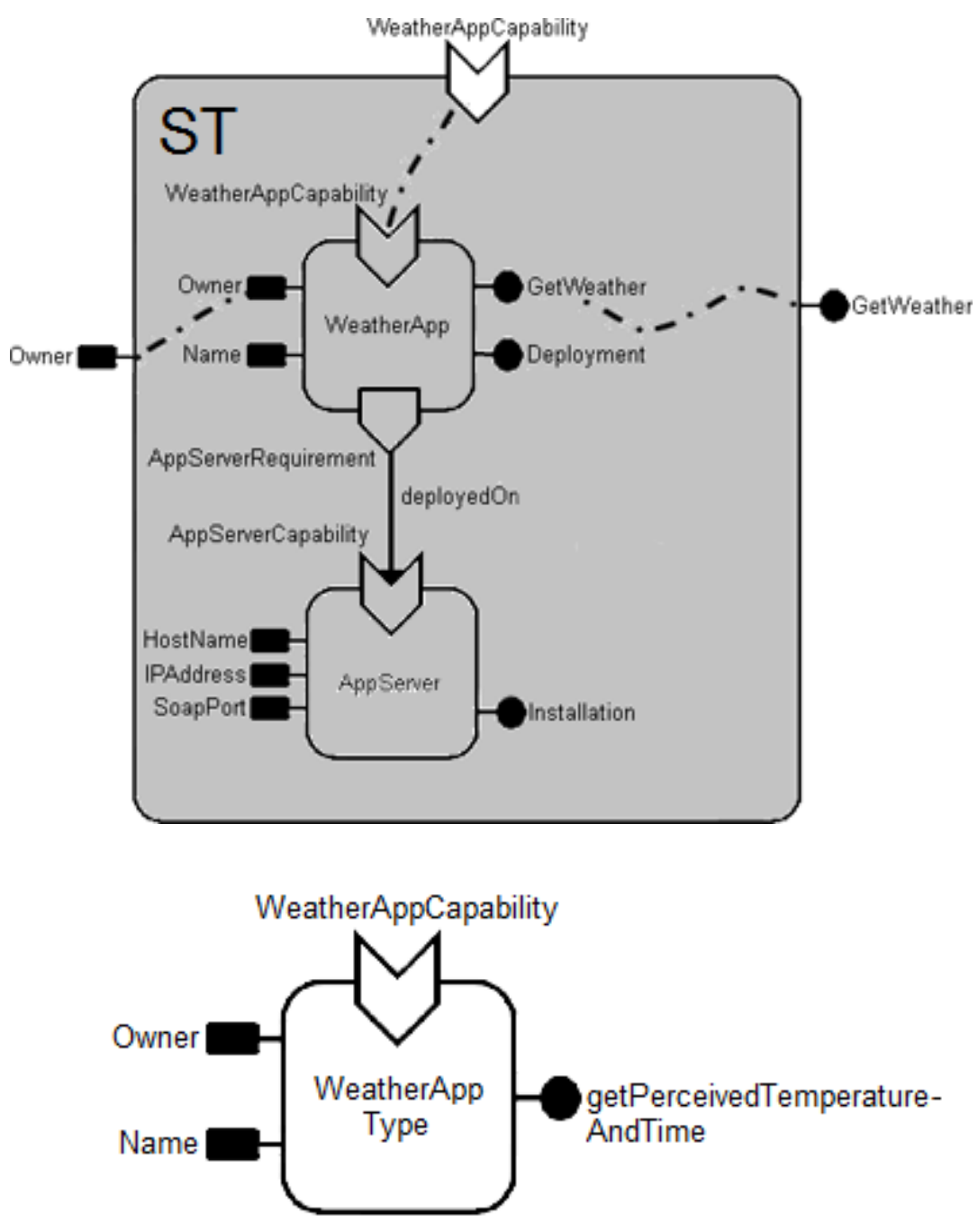

Figure 4.8: A complete matching example. 
Subsection 4.2, will not be a signature one. Indeed, we will use the crossontology approach to find a property which is semantically equivalent to the required one. So, suppose that the desired Name property is semantically equivalent to the available HostName property of AppServer. Then, we have to modify $S T$ boundaries in order to expose also the desired (renamed) property.

Once the property search is done, we have to proceed in looking for the desired operation. Suppose that the condition

$$
\begin{gathered}
\forall \text { Operation } x \in \text { N.Interfaces.Interface } \\
\exists \text { Operation } y \in \text { ST.Interfaces.Interface: } \\
x \cong_{O} y
\end{gathered}
$$

does not hold. This means that we have to employ the OpERATIONSETSDISCOVERING algorithm in order to check whether some available operation combinations let us satisfy the desired following condition.

$$
\begin{gathered}
\exists \text { Plan } p \in O C_{S T}: \\
x \cong_{O}(\text { Operation }) p .
\end{gathered}
$$

So, following a behaviour analogue to the one of the example in Subsection 4.3.3, we obtain the desired plan (see Figure 4.7). Now, we have to modify $S T$ boundaries in order to exhibit the obtained plan as an operation semantically equivalent to the desired one.

Please note that, once the above modifications has been performed, we obtain a (new) ServiceTemplate $S T^{\prime \prime}$ which is in black-box flexible matching with the desired Weather AppType NodeType. So, we simply have to employ the (extended) oblivion boundaries adaptation approach in order to obtain the Service Template $S T^{\prime}$ which can be used as a substitute for the desired NodeType (see Figure 4.9). 


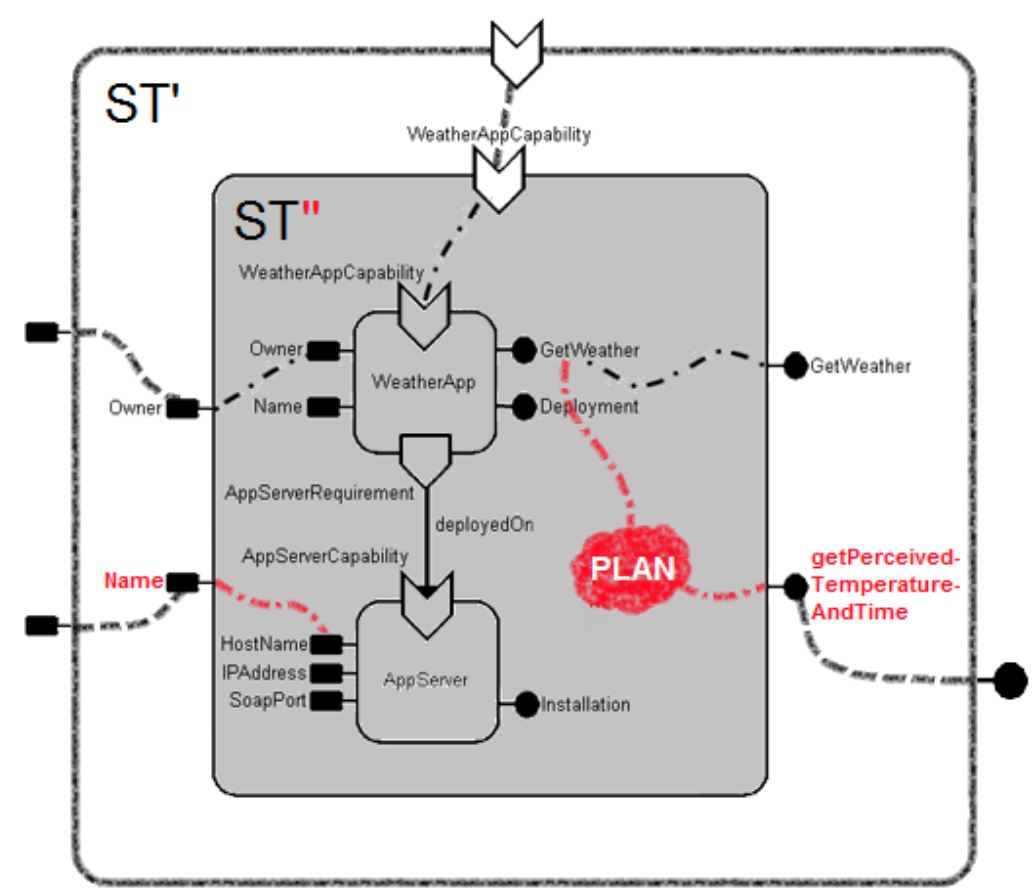

Figure 4.9: Complete matching example adaptation.

\subsubsection{Matching and adaptation}

As shown in Figure 4.10, all the matching procedure (both the blackbox part and the white-box one) is completely automatable. Using this procedure we can generate the desired ServiceTemplate $S T^{\prime}$ (i.e., the one which can be used as a substitute for the needed NodeType $N$ ) by adapting the available $S T$. What kind of adaptation is performed?

While in the black-box matching case the adaptation consists (at most) in filtering and renaming the available feature, the white-box matching situation is more complicated. We have seen that the operations to be performed are the following ones: extracting (inside) available features and generating plans (if needed). So, referring Figure 3.11, the adapter $A$ must implement the above mentioned functionalities. 


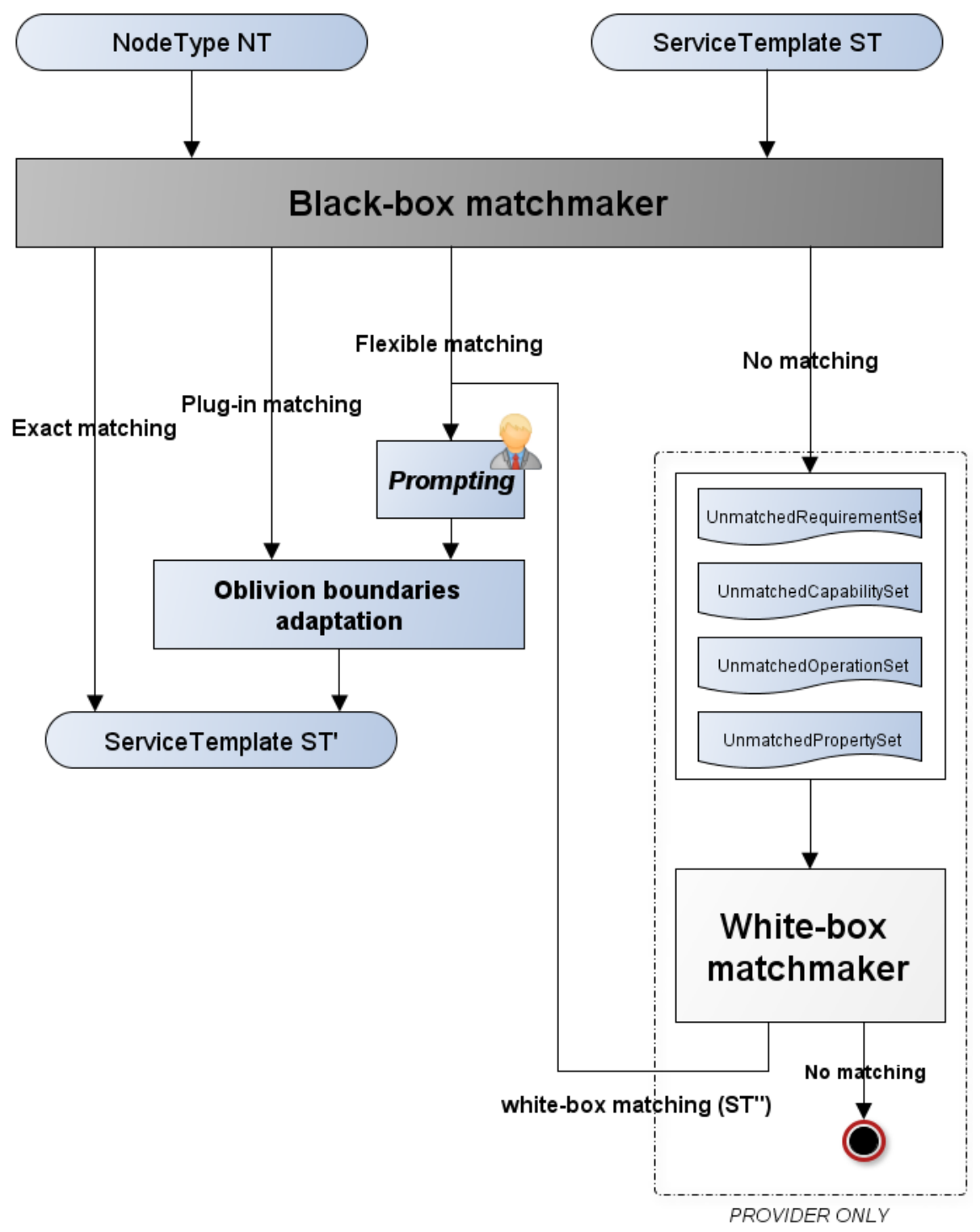

Figure 4.10: Extended matching (and adaptation) procedure. 
Chapter 4. NodeType white-box matching (and adaptation)

\subsubsection{Taking semantics into account}

As for the flexible matching approach, the white-box viewpoint procedure exploits ontologies to obtain the desired matchings. More precisely, ontologies are used to:

- pair (semantically) equivalent features, and

- generate plans (which can be used as substitutes for missing operations - if possible).

Please note that the above operations exactness strictly depends on both the input ontologies and the cross-ontology matchmaker. It follows that, if those elements are not so accurate as required, some generated pairings/plans could not be significant and should not be used in our matching (and adaptation) procedure.

As shown in Figure 4.10, when the considered NodeType $N$ whitebox matches with the ServiceTemplate ST, we have to prompt to the user the white-box matchmaker decisions taken to transform $S T$ in a ServiceTemplate $S T^{\prime \prime}$ which flexible matches $N$. If she accepts those decisions (along with those taken with the flexible matching procedure), then we can proceed in developing the adapted ServiceTemplate $S T^{\prime}$ via the oblivion boundaries approach.

It is worth noting that the way in which the white-box decision prompting is performed strictly depends on the matchmaker implementation. We recommend to start by submitting to the user the decisions which are most probable and to let the user mark, adjust or discard the wrong decisions. 


\subsubsection{Practical observations}

It is worth making some remarks on the usability of matching methodologies explained in Chapters 3 and 4 . The black-box matching procedure employs the available ServiceTemplate ST without doing any modification on its specification. This means that the adapter $A$ (see Figure 3.11) could be developed by both the service client and the service provider. Conversely, the white-box matchmaker (possibly) requires to update $S T$ boundaries and to generate plans of available operations. In other words it needs to work inside of $S T$ (as recalled by the methodology name). So, as reported in Figure 4.10, the only one who can exploit this method to obtain the adapted ServiceTemplate $S T^{\prime}$ is the service provider.

Since the white-box matching procedure can only be executed by the service provider, the execution environment is a cloud one. This implies that this procedure can (ideally) count on an infinite amount of both computational power and storage space. So, as already mentioned in Subsections 4.3.1 and 4.3.2, this huge amount of resources could be employed to decrease significantly the time complexity of the matching and adaptation procedure (pre-computing and storing the dependency hypergraph and parallelizing the OPERATIONSETSDISCOVERING implementation).

\subsubsection{What's next?}

So far, we have explained a way to match NodeType and ServiceTemplate elements from both a black-box viewpoint (Chapter 3) and a white-box one (Chapter 4).

Please recall that the objective of this thesis is to find a way to check whether available (TOSCA-compliant) services can be used as NodeTemplate components of a TOSCA multi-cloud ServiceTemplate. So, the presented matchings between node types and service templates can be seen 
as type-matchings. Those checking (and adaptation) procedures can be used only in the first step of the desired complete checking. Which is the second (and last) one? Once the type-checking is satisfied, we have to compare values in order to determine if they are compatible. Then, as we will state in Chapter 6, a way to obtain the desired value comparison between the considered TOSCA elements must be individuated. 


\section{Chapter 5}

\section{Proof-of-concept implementation}

As mentioned in Chapter 1 , the different types of matching defined in this thesis should be used to develop a matchmaker to be fruitfully integrated in the TOSCA implementations that are currently under development. In order to enforce this observation, this Chapter 5 aims at showing the feasibility of such (pluggable) matchmaker.

This chapter will start by showing a possible implementation of the TOSCA basic features (viz., capabilities, requirements, policies, properties and interface operations - Section 5.1). Then, in Section 5.2, such features are opportunely grouped to let the needed TOSCA elements be implemented (viz., NodeType and ServiceTemplate elements). Afterwards, with Section 5.3 , a possible implementation of the exact and plugin matchmakers is given. Finally, Section 5.4 provides some concluding remarks.

All the following listings contain JAVA source code. The reason why we select JAVA as source code language resides in two main factors:

- the TOSCA specification implements inheritance in a way strictly 
similar to that of object-oriented languages, and

- the only TOSCA implementation currently available (see [33]) is written in JAVA.

\subsection{Implementation of basic features}

This section aims at showing how the TOSCA basic features can be implemented. So, in the following paragraphs we will show a possible realization of capabilities, requirements, policies, properties and interface operations.

\section{Implementation of capabilities}

We know that each component capability is identified via its name and its type. As we observe in Listing 5.1, how to name the desired capability is not a problem: we simply have to equip the capability class with a name field. But, how can we deal with the typing problem? It is worth pointing out that, since we define the abstract CapabilityType class (which represents the generic characteristics of a capability type), we can make each desired capability type correspond to a class derived from the CapabilityType one. So, the capability typing comes naturally with the class instantiation.

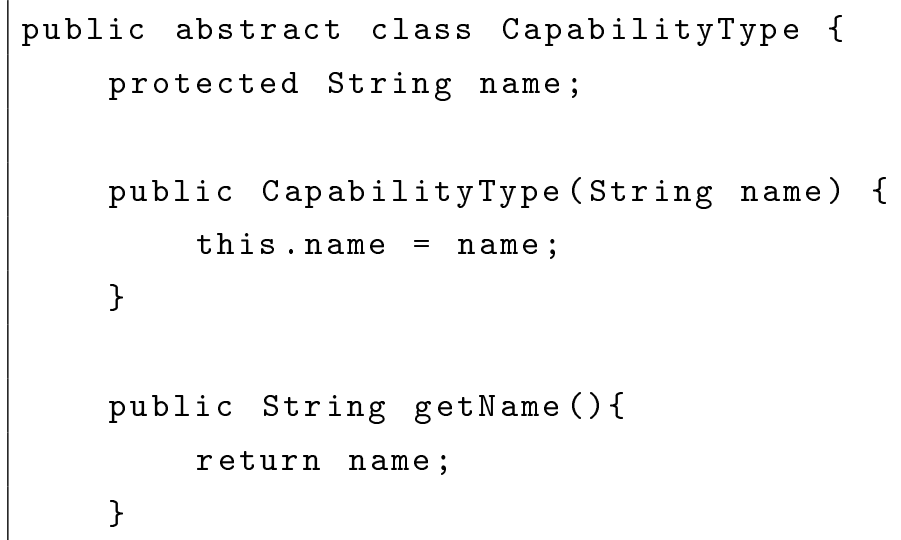




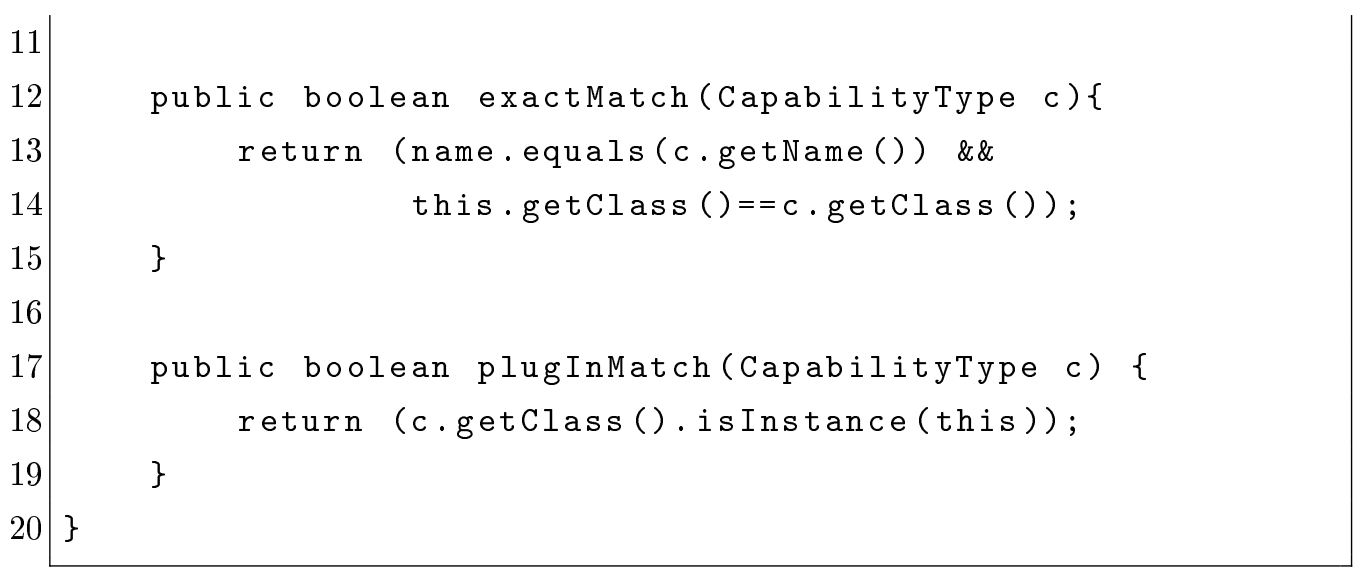

Listing 5.1: JAVA implementation of a generic capability (type).

Please note that the provided CapabilityType class is equipped with two non-obvious methods: exactMatch (lines 12-15) and plugInMatch (lines 17-19). Such methods are used in the similar-named matching procedures (since they return a boolean which represents whether the current capability type and the passed one satisfy the exact or plug-in 1 matching condition, respectively).

\section{Implementation of requirements}

As we already mentioned in Chapter 2, there is a high similarity between capability and requirement structures. So, the requirement JAVA implementation is analogous to the capability one (see Listing 5.2.

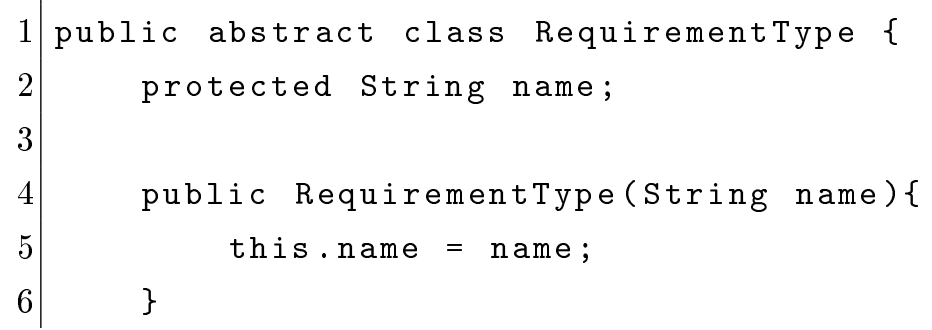

\footnotetext{
${ }^{1}$ Please remember that with the presented implementation the type of a capability corresponds to the instantiated class. So, what we have to do (to check whether the current capability is of a type derived from that of the passed one) is to check whether the current object can be considered an instance of the passed object class.
} 


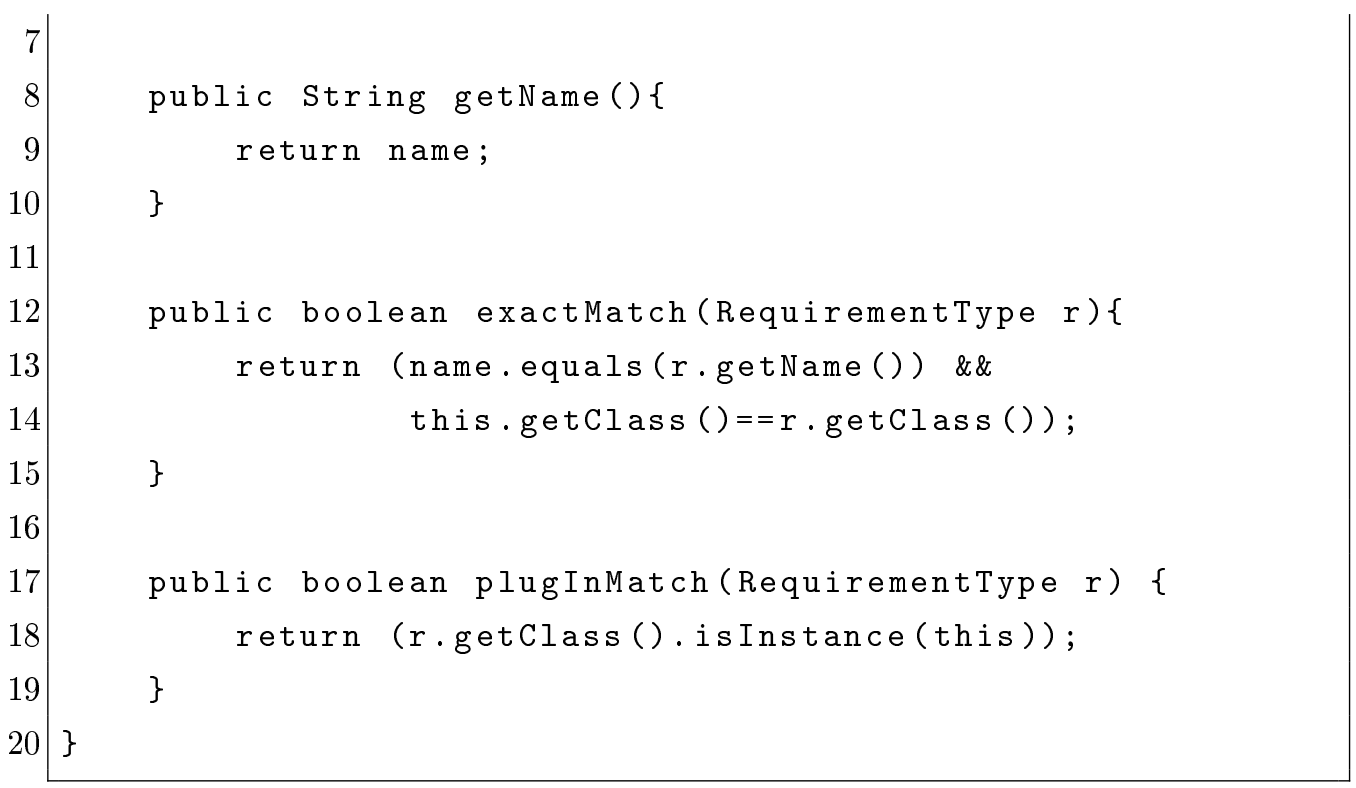

Listing 5.2: JAVA implementation of a generic requirement (type).

\section{Implementation of policies}

Please recall that (as discussed in Chapter 2) we identify each policy via its own type. As for requirements and capabilities, we decide to implement such a policy typing via the definition of a generic PolicyType (Listing 5.3. Such a generic element is an abstract class which contains all the features common to all the definable policy types (viz., the set of nodes to which it is applicable and some management methods).

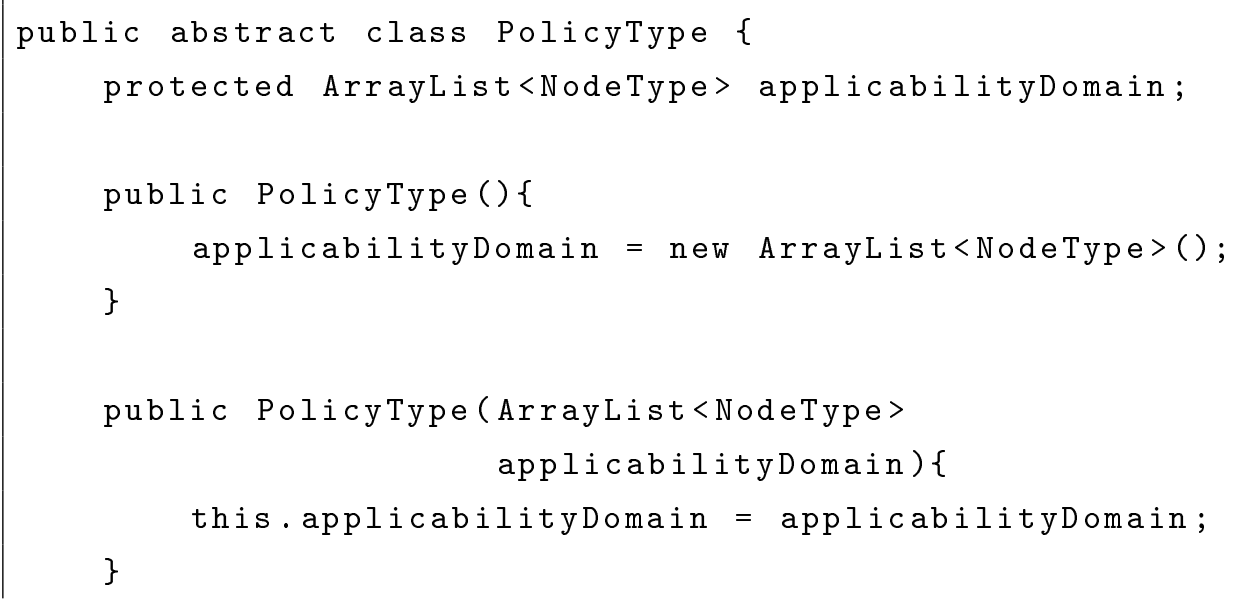




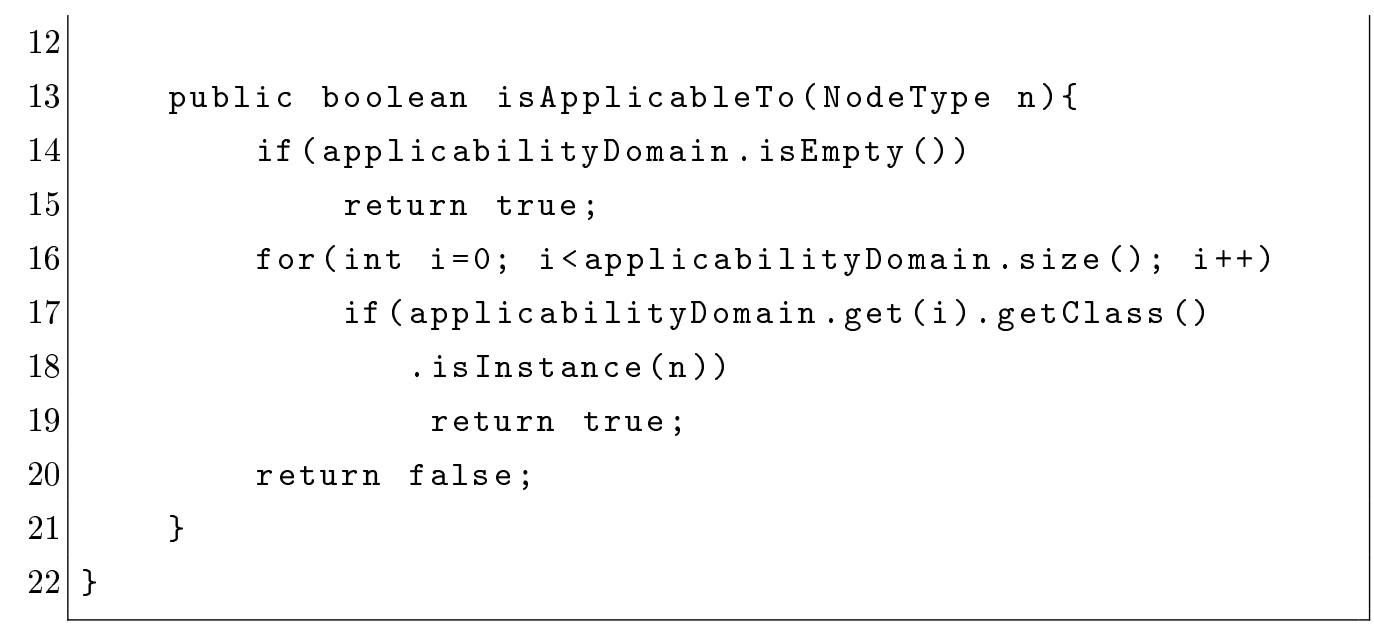

Listing 5.3: JAVA implementation of a generic policy (type).

Please focus on method isApplicableTo (lines 13-21). As anticipated by its name, such method is used to check whether the current policy is applicable to the argument node $\mathrm{n}$ (of a certain NodeType - see Subsection 5.2. The applicability checking consist of verifying whether:

- the current policy type is applicable to every node type (lines 14$15)$, or

- the (current policy type) set of applicable node types contains a NodeType $N$ equal to n's type (or from which it is derived - lines 16-19).

So, we can represent each TOSCA PolicyType with a class derived from the PolicyType one. Now, since policies matching is quite different from other feature matching, we decided to represent the whole policy set (of a node/service template) with a separated Policies class (Listing 5.4 .

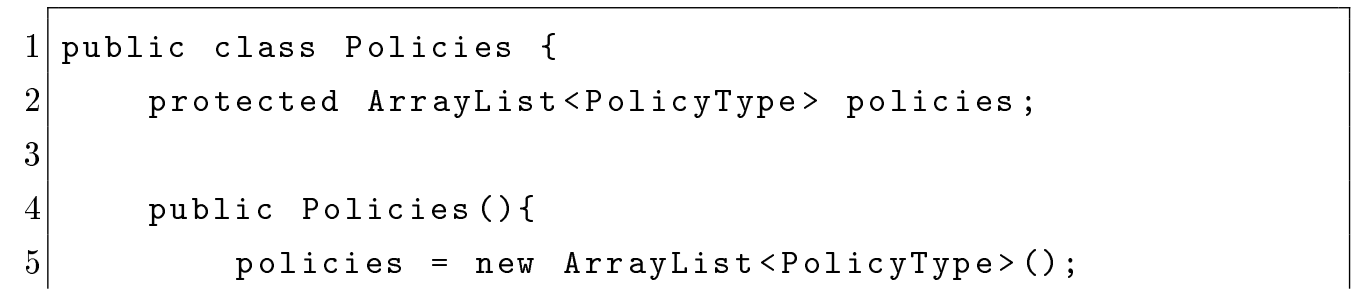




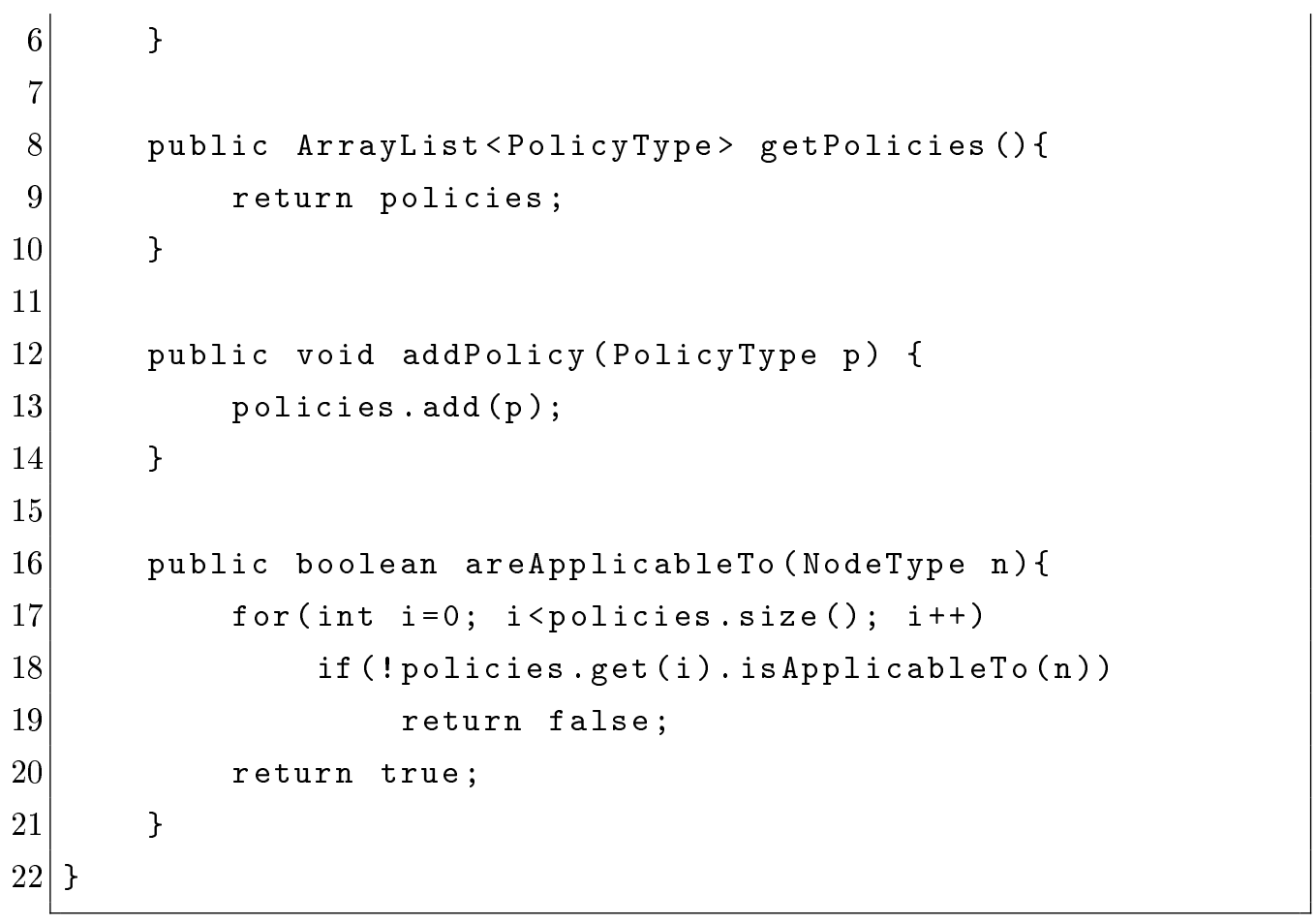

Listing 5.4: JAVA implementation of a set of policies.

The reason why we decided to separate such policy set implementation mainly resides in the possibility of defining the areApplicableTo method. This method implements the operator $\equiv_{P O}$ (which checks whether the current set of policies is applicable to a certain NodeType - see Section 3.1 .

\section{Implementation of properties}

We know that each component property can be seen as a pair

$$
\langle\text { name,value }\rangle \text {. }
$$

It is worth noting that, according to our type-checking purposes, taking care of the value type is quite important. So, as done before, such a type is related to a JAVA class. Please observe that, while for capabilities, requirements and properties we needed to define a generic class (viz., 
type), here we already have it. Indeed, since we are considering "real" values, we need to employ the (generic) Object class.

The above reported observations let us implement a TOSCA property as shown in Listing 5.5 .

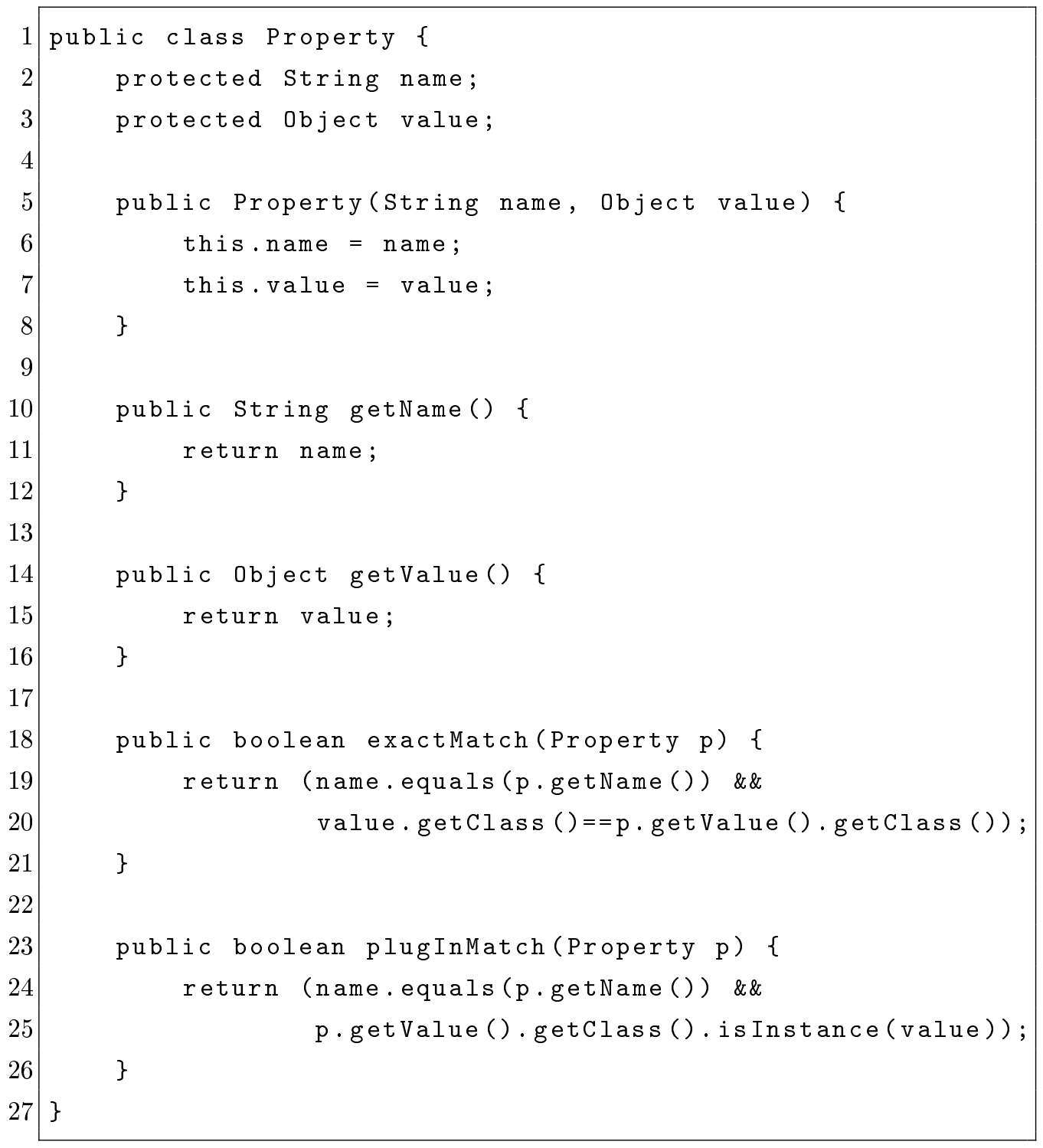

Listing 5.5: JAVA implementation of a property.

Please note that (as was for CapabilityType and RequirementType classes) the Property class is equipped with the methods exactMatch and plugInMatch. As before, such method returns a boolean which 
represents whether the current property and the passed one satisfy the exact and plug-in ${ }^{2}$ (single property) matching condition, respectively.

\section{Implementation of interface operations}

TOSCA component interfaces implementation requires the realization of three distinct elements: parameters, operations and interfaces. In the following, we will use a bottom-up to show such implementations.

The first element to be modeled is the operation parameter. According to the TOSCA specification [25], we implement such a parameter as a triple

$$
\langle\text { name,value,required }\rangle
$$

where name is the parameter name, value is its value and required is a truth value which indicates whether the user must specify the parameter value. As before, our type-checking approach requires to take care of value's type. So, we make it correspond to the class of the value object.

The above stated consideration let us implement the operation parameters via the OperationParameter class (Listing 5.6).

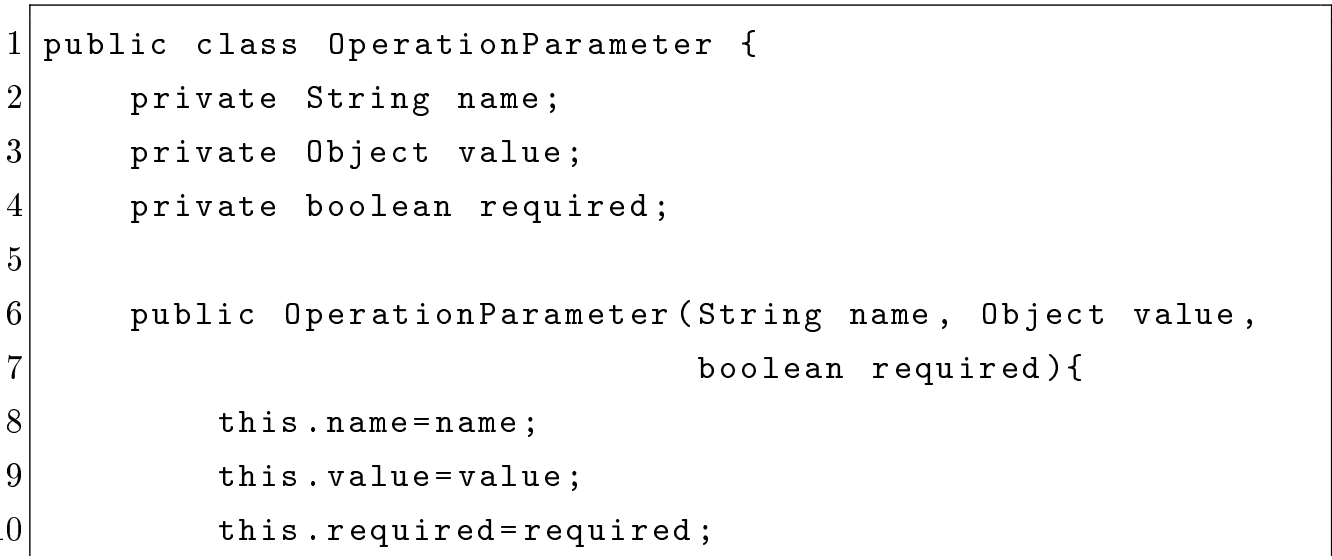

\footnotetext{
${ }^{2}$ Please recall that the property value type corresponds to a JAVA class. So, to obtain the plug-in matching we need to ensure that the current property value is an instance of the passed property value class.
} 


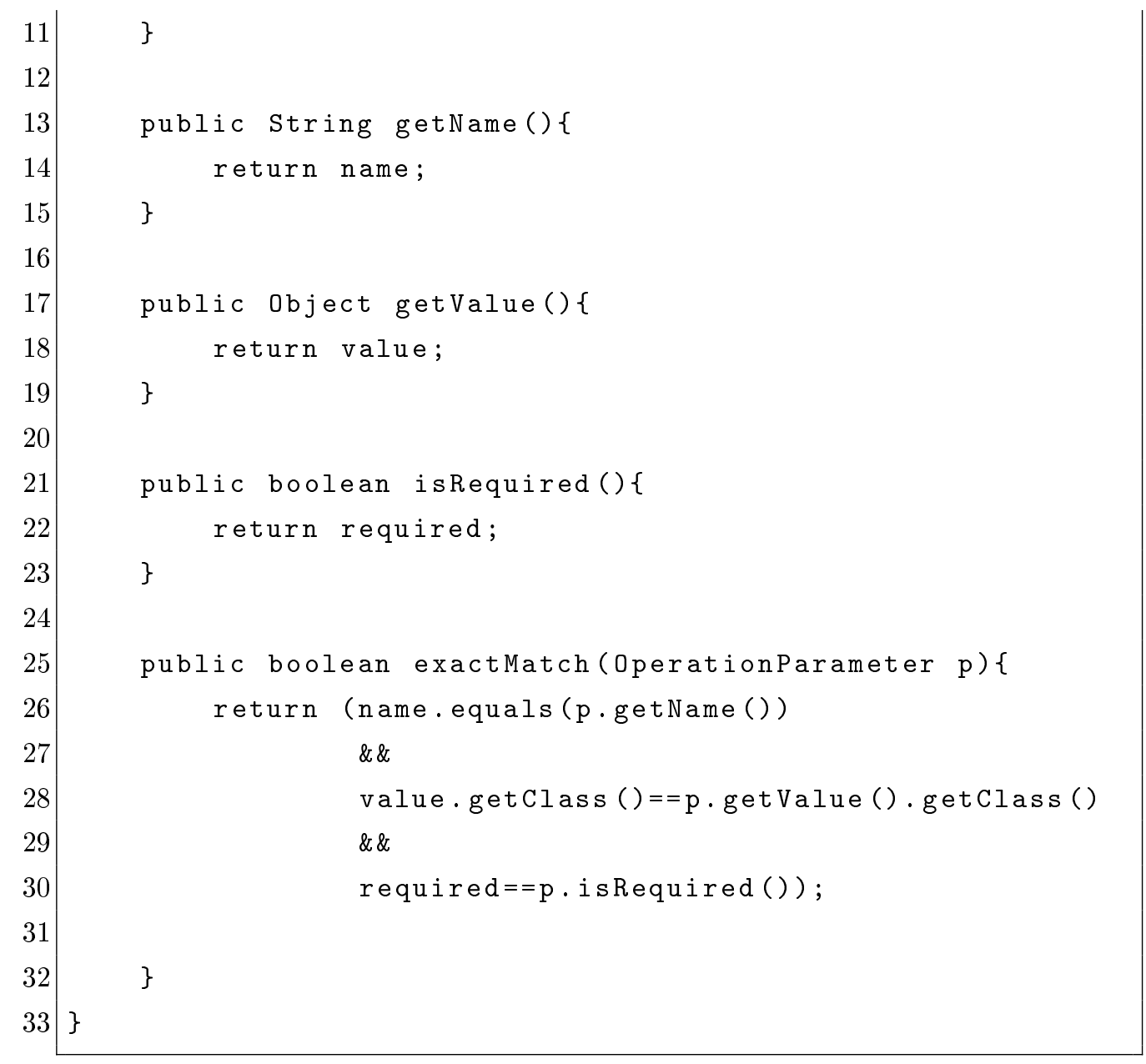

Listing 5.6: JAVA implementation of an operation parameter.

It is worth noting that, despite we perform both exact and plug-in matching, operation parameters only require to implement the exactMatch operation (which checks whether the current parameter and the passed one satisfy the exact matching condition). This is because, looking at the definitions in Subsections 3.1 and 3.2, we observe that both the two matching levels employ the exact matching between parameters.

Once parameters have been implemented, we can move on with operation implementation. Intuitively speaking, each operation can be viewed as a triple

〈name, inputParameters,outputParamters〉 
where name is the operation name, inputParameters and outputParameters are the set of input and output parameters, respectively. So, we can model such operation with the class of Listing 5.7 .

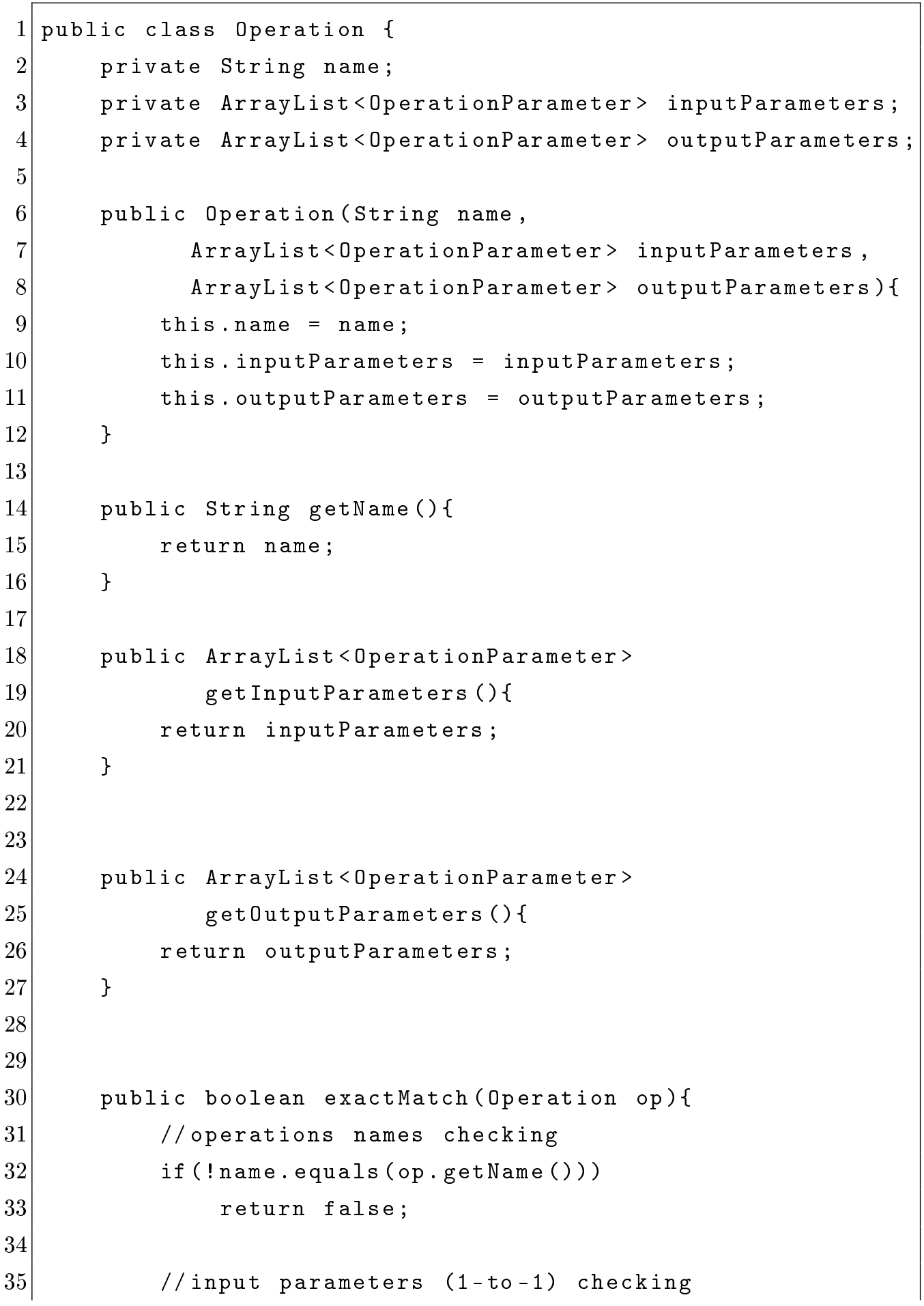




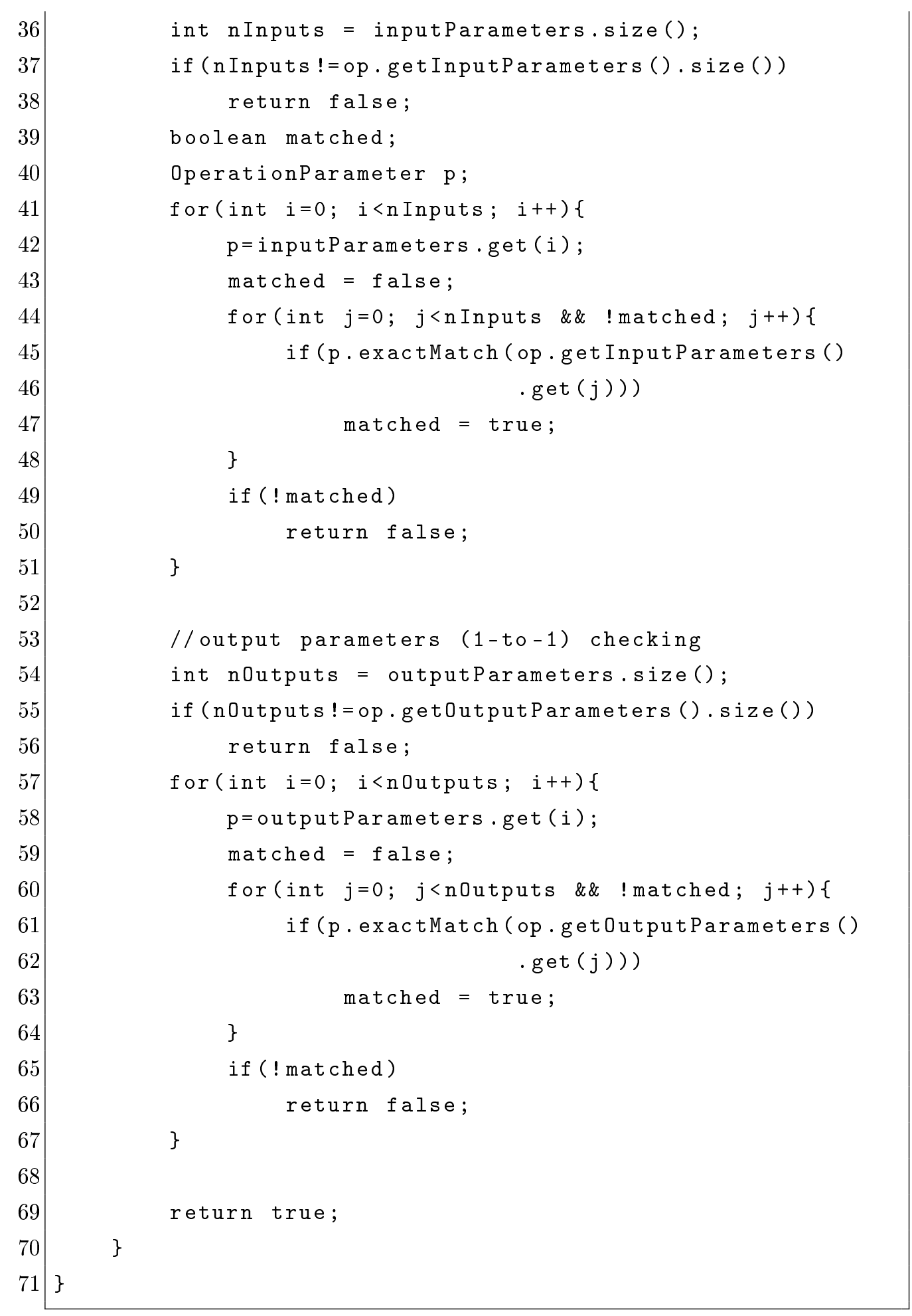

Listing 5.7: JAVA implementation of an operation parameter. 
As for parameters, both the exact and plug-in matching employ the operations exact matching. So, only the exactMatch method is developed. Let us now focus on such a method. It starts by checking whether the two operations are same-named (lines 31-33). Afterwards, it proceeds by looking for a one-to-one correspondence between the two operations input parameters (lines 35-51) and between their output parameters (lines 53-67). If none of the previous matchings fail, then it returns true (since the two operations can be considered exactly matched - line 69).

Let us now consider the interface implementation. As mentioned in Chapter 2, each interface can be viewed as a pair

\section{$\langle$ name,operations $\rangle$}

where name is the interface name and operations is the set of interface operations. This let us implement such a TOSCA basic feature as reported in Listing 5.8 .

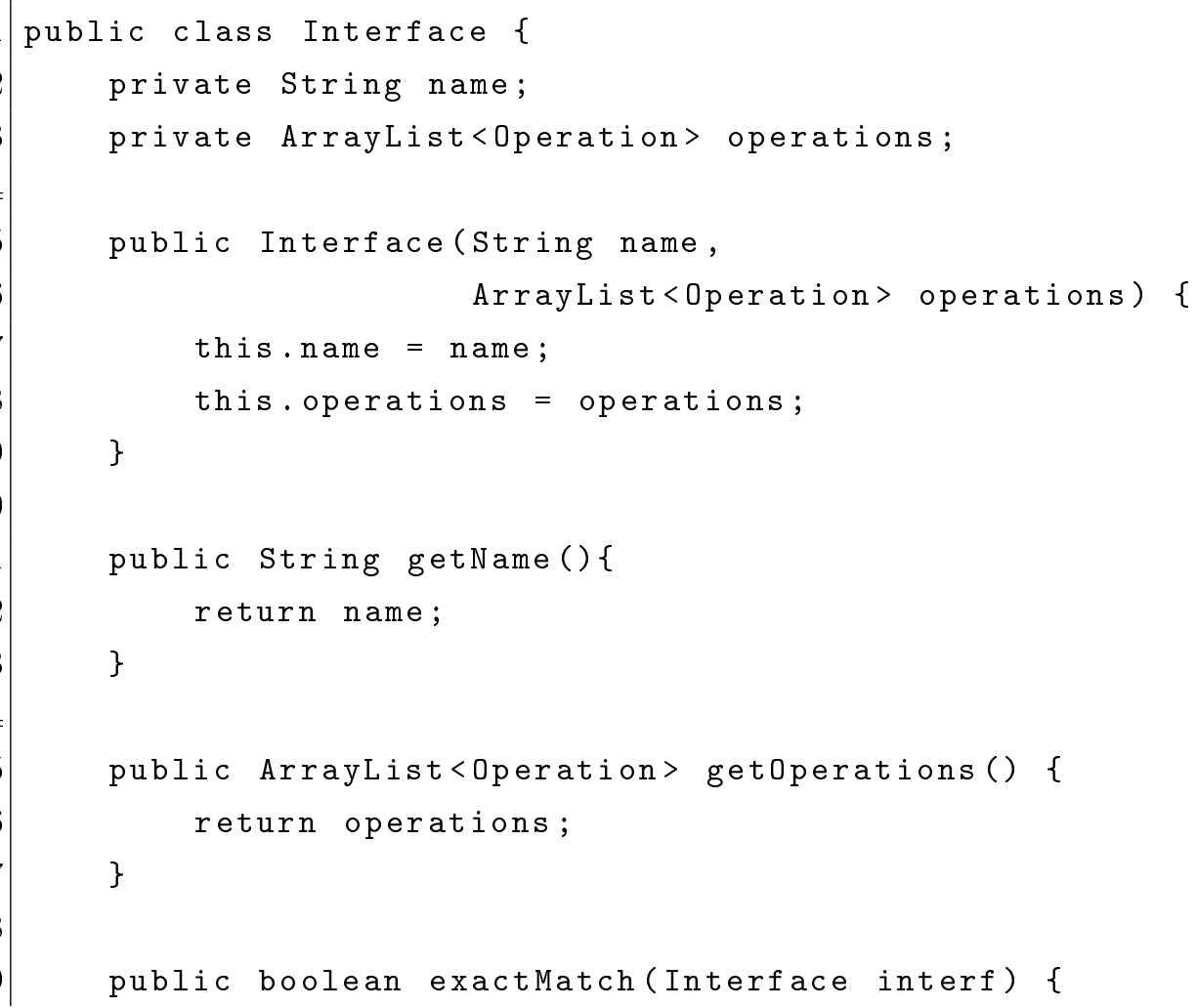




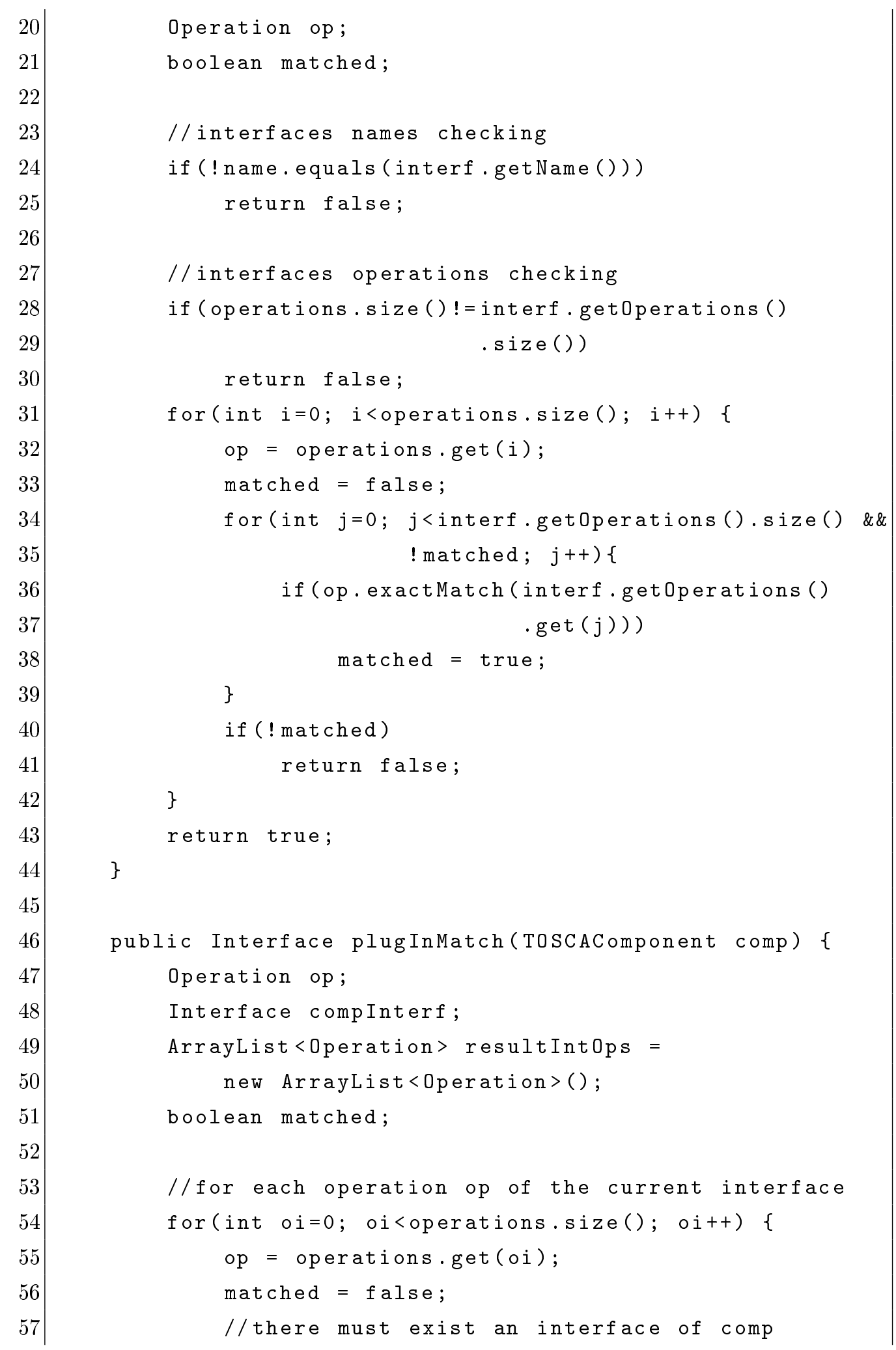




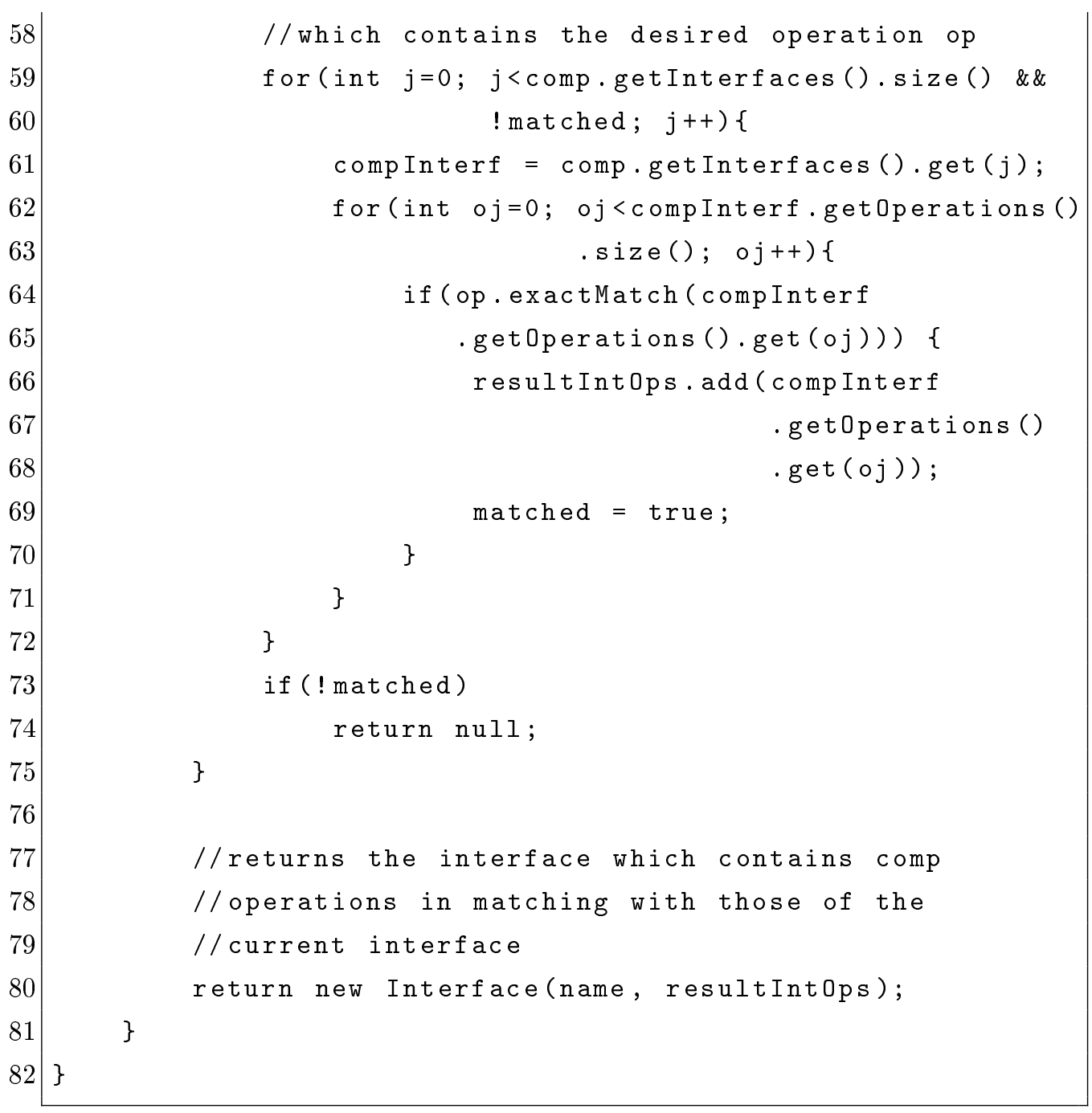

Listing 5.8: JAVA implementation of an interface.

As done for previous elements, Interface is equipped with two methods (exactMatch and plugInMatch) which let the user check whether the current interface exactly matches the passed one and whether the current interface plug-in matches the interfaces of the passed TOSCAComponent? respectively. It is worth noting that, since the matching is performed in order to develop an adapted service template, the matched operations of the passed component are grouped in order to obtain a new interface which exactly matches the current one. Such a new interface is then

\footnotetext{
${ }^{3}$ See Section 5.2
} 
returned 4

\subsection{Implementation of the needed TOSCA components}

Once the basic TOSCA features (viz., capabilities, requirements, policies, properties and interface operations) have been developed, we can proceed in implementing the TOSCA NodeType and ServiceTemplate elements. So, the following paragraphs aim at illustrating (a sketch of) how to develop in JAVA such TOSCA elements.

Please remember that we are going to implement a sketch of the matching procedure proposed in this thesis (in order to show its - JAVA - feasibility). More precisely, what we are going to do is to implement the black-box exact and plug-in matching notions. So, to our purposes, restricting the view of a TOSCA service component to only what it exposes is enough $\bigsqcup^{5}$.

\section{Abstract TOSCAComponent class}

The above simple observation let us model TOSCA service components as classes derived from the abstract TOSCAComponent one (Listing 5.9).

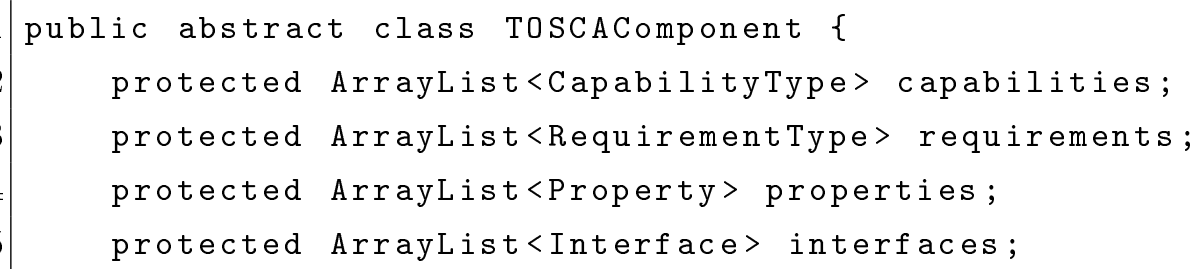

${ }^{4}$ If the matching fails, then a null interface is returned.

${ }^{5}$ Furthermore, assuming to look at TOSCA service component from a black-box viewpoint is not so restricting as someone could think. All of the TOSCA IDE will probably let users look at service components from both the black-box and white-box viewpoint. 


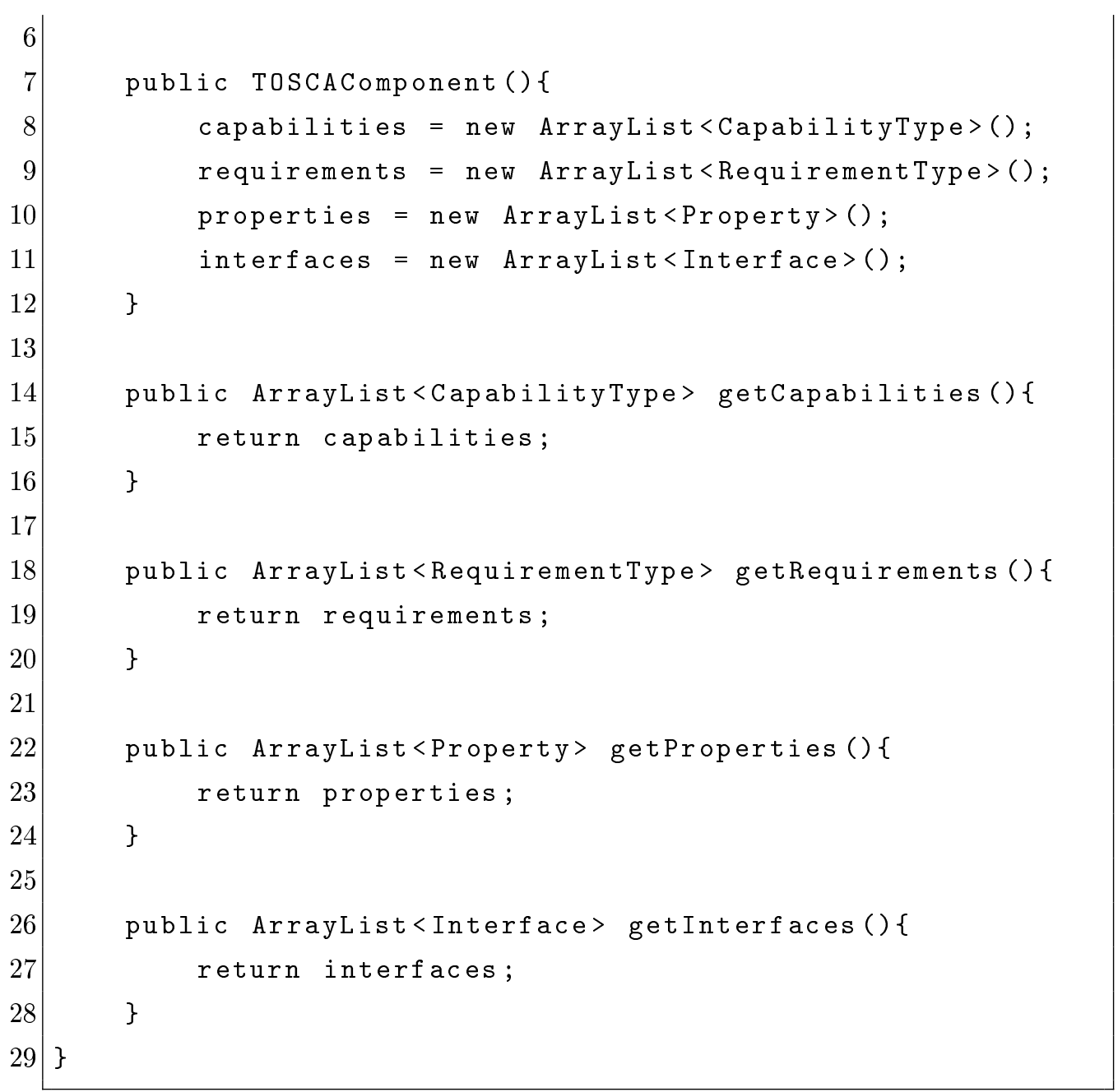

Listing 5.9: JAVA implementation of a generic service component.

Intuitively speaking, the above reported JAVA class let us model the elements common to all the TOSCA service components. Furthermore, this let us use such an abstract class as a generic type to be passed to such methods which are for instance applicable to both NodeType and ServiceTemplate elements (e.g., the plugInMatch method of the Interface class). 


\section{NodeType implementation sketch}

To our type-checking purposes, a generic NodeType can be viewed as a quadruple

$$
\langle\text { capabilities,requirements,properties,interfaces }\rangle .
$$

Please note that all the needed features are already contained in the TOSCAComponent class. So, we can develop the generic NodeType simply extending the above mentioned class (Listing 5.10).

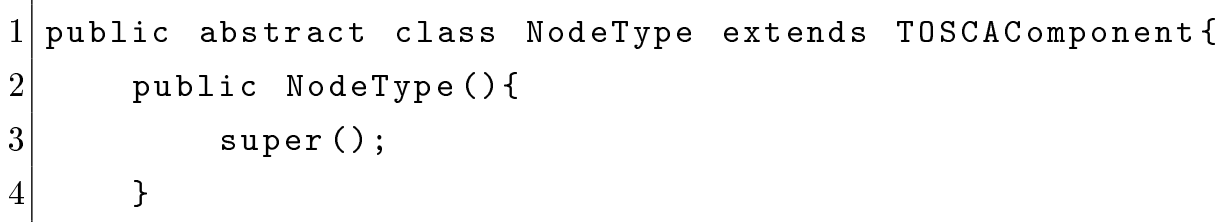

Listing 5.10: JAVA implementation of a generic NodeType.

Once the generic (viz., abstract) NodeType has been developed, our matchmaker user simply needs to declare each desired node type as derived from it.

\section{ServiceTemplate implementation sketch}

Differently from NodeType elements, a ServiceTemplate could also expose policies. So, it extends the generic TOSCAComponent adding such a field (with its own management methods - Listing 5.11).

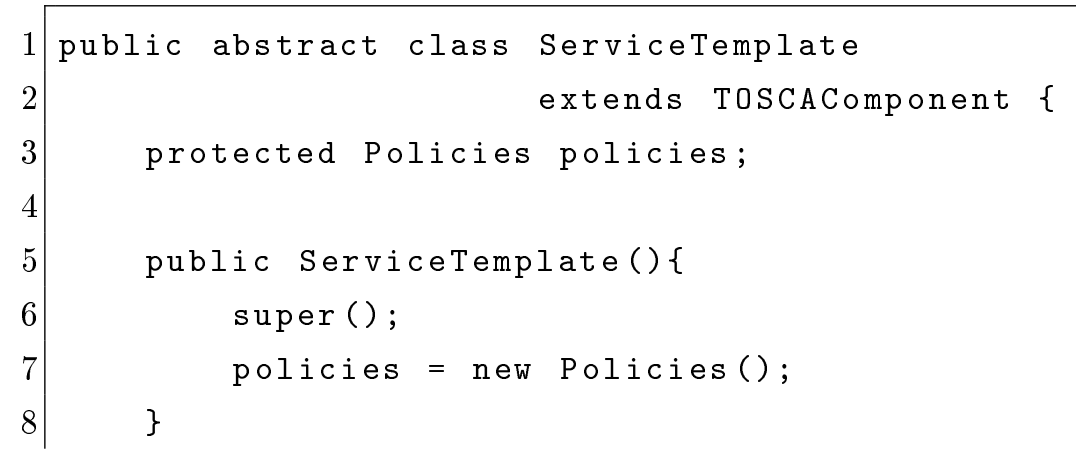




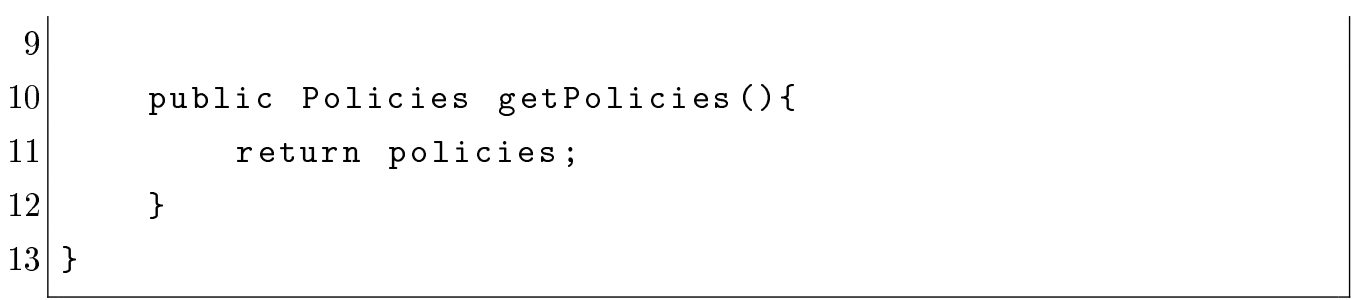

Listing 5.11: JAVA implementation of a generic Service.

As was for NodeType elements, our matchmaker user simply needs to declare each desired service template as derived from the generic (viz, abstract) ServiceTemplate.

It is worth noting that, while both NodeType and ServiceTemplate can be used as substitutes for the generic TOSCAComponent, thanks to JAVA inheritance they are two distinct elements. Such a distinction is made in order to not break the rules of the TOSCA specification [25].

\subsection{Implementation of the matchmakers}

Sections 5.1 and 5.2 have provided all the ground needed to implement the exact and plug-in matchmakers. To develop such matchmakers, we will follow the same extensive approach used in Chapter 3 . In other words, we will develop the desired matchmakers following the class hierarchy of Figure 5.1.

\section{Abstract Matchmaker class}

Before giving the employable matchmakers, we provide an abstract Matchmaker (Listing 5.12 which defines the common characteristics of each deployed matchmaker.

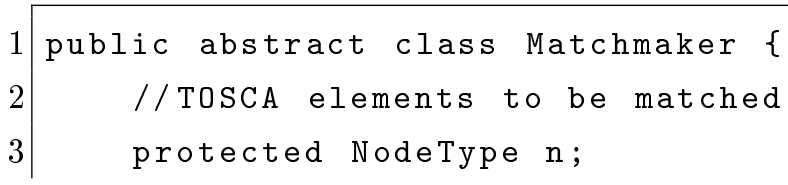




\begin{tabular}{|c|}
\hline $\begin{array}{l}\text { <<abstract }>> \\
\text { Matchmaker }\end{array}$ \\
\hline $\begin{array}{l}\text { \# n : NodeType } n \\
\text { \# st: ServiceTemplate } \\
\\
\text { \# capMatchResult : Result } \\
\text { \# unmatchedCapabilities : ArrayList<CapabilityType> } \\
\text { \# reqMatchResult : Result } \\
\text { \# unmatchedRequirements : ArrayList<RequirementType> } \\
\text { \# polMatchResult: Result } \\
\text { \# propMatchResult : Result } \\
\text { \# unmatchedProperties : ArrayList<Property> } \\
\text { \# intMatchResult: Result } \\
\text { \# unmatchedlnterfaces : ArrayList<Interface> }\end{array}$ \\
\hline $\begin{array}{l}\text { + Matchmaker(n : NodeType, st: ServiceTemplate) } \\
\text { + getUnmatchedCapabilities } 0: \text { ArrayList<CapabilityType> } \\
\text { + getUnmatchedRequirements } 0: \text { ArrayList }<\text { RequirementType }> \\
\text { + getUnmatchedProperties } 0: \text { ArrayList }<\text { Property> } \\
\text { + getUnmatchedinterfaces } 0: \text { ArrayList }<\text { Interface> } \\
\text { + match } 0: \text { Result }\end{array}$ \\
\hline \\
\hline ExactMatchmaker \\
\hline $\begin{array}{l}\text { \# exactMatchedCapabilities : ArrayList }<\text { CapabilityType }> \\
\text { \# exactMatchedRequirements : ArrayList }<\text { RequirementType }> \\
\text { \# exactMatchedProperties : ArrayList }<\text { Property> } \\
\text { \# exactMatchedinterfaces : ArrayList }<\text { Interface > }\end{array}$ \\
\hline $\begin{array}{l}\text { + ExactMatchmaker(n : NodeType, st: ServiceTemplate) } \\
\text { + getExactMatchedCapabilities } 0: \text { ArrayList<CapabilityType> } \\
\text { + getExactMatchedRequirements } 0: \text { ArrayList<RequirementType> } \\
\text { + getExactMatchedProperties } 0: \text { ArrayList }<\text { Property> } \\
\text { + getExactMatchedInterfaces } 0: \text { ArrayList }<\text { Interface> } \\
\text { - exactMatchCapabilities } 0: \text { Result } \\
\text { - exactMatchRequirements } 0: \text { Result } \\
\text { - exactMatchPolicies } 0: \text { Result } \\
\text { - exactMatchProperties } 0: \text { Result } \\
\text { - exactMatchInterfaces } 0: \text { Result } \\
\text { + match0: Result }\end{array}$ \\
\hline \\
\hline PlugInMatchmaker \\
\hline $\begin{array}{l}\text { \# pluginMatchedCapabilities : ArrayList }<\text { CapabilityType }> \\
\text { \# pluginMatchedRequirements : ArrayList }<\text { RequirementType }> \\
\text { \# pluginMatchedProperties : ArrayList }<\text { Property }> \\
\text { \# pluginMatchedInterfaces : ArrayList }<\text { Interface }>\end{array}$ \\
\hline $\begin{array}{l}\text { + PlugInMatchmaker(n : NodeType, st : ServiceTemplate) } \\
\text { + getPlugInMatchedCapabilities } 0: \text { ArrayList }<\text { CapabilityType> } \\
\text { + getPlugInMatchedRequirements } 0: \text { ArrayList }<\text { RequirementType> } \\
\text { + getPlugInMatchedProperties } 0: \text { ArrayList }<\text { Property> } \\
\text { + getPlugInMatchedinterfaces } 0: \text { ArrayList }<\text { Interface> } \\
\text { - plugInMatchCapabilities } 0: \text { Result } \\
\text { - plugInMatchRequirements } 0: \text { Result } \\
\text { - plugInMatchPolicies } 0: \text { Result } \\
\text { - plugInMatchProperties } 0: \text { Result } \\
\text { - plugInMatchInterfaces } 0: \text { Result } \\
\text { + match0: Result }\end{array}$ \\
\hline
\end{tabular}

Figure 5.1: UML diagram of the developed matchmakers. 


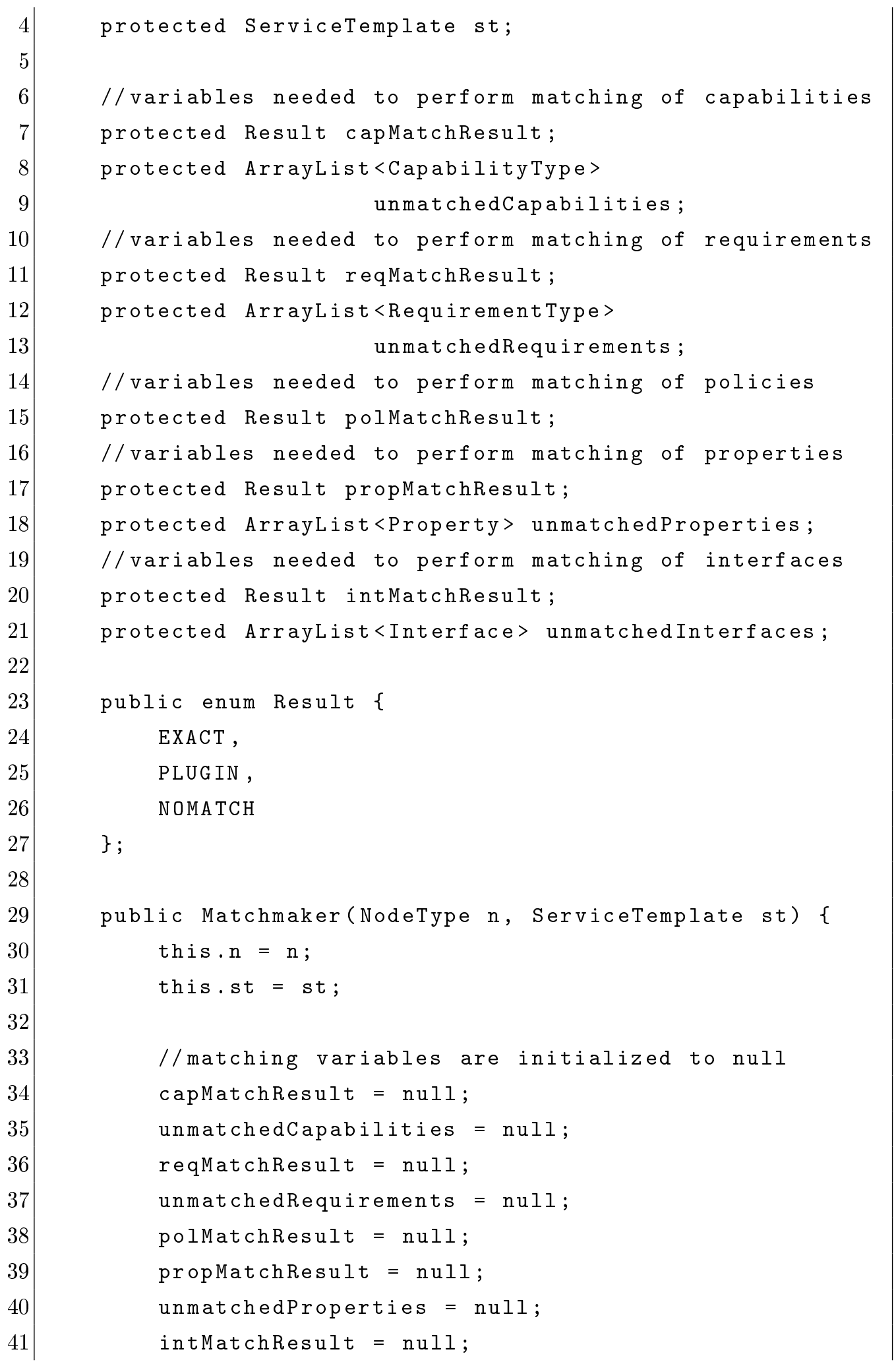




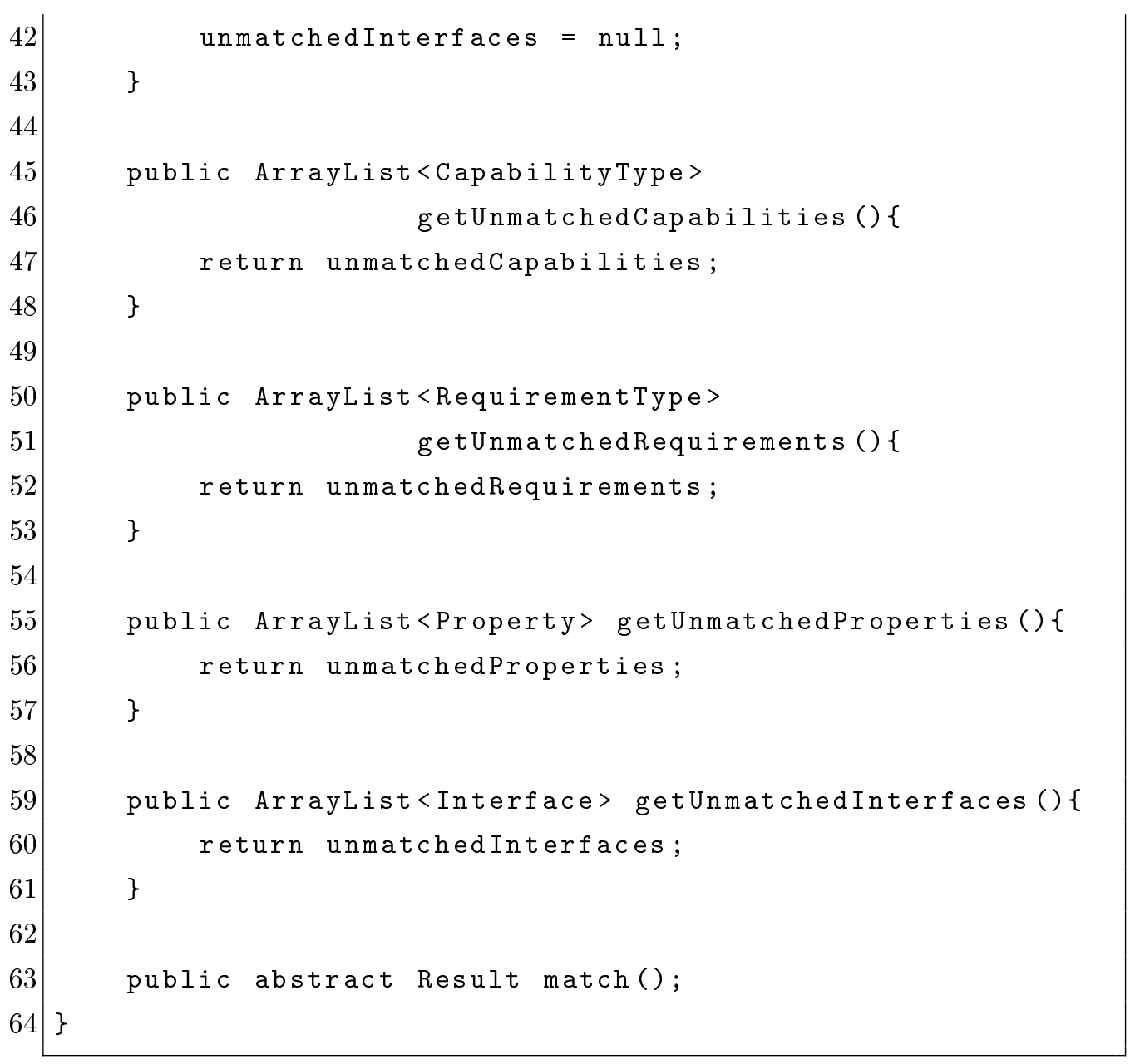

Listing 5.12: JAVA implementation of the abstract Matchmaker.

Please note that for each basic feature (to be matched) we define two fields: result and unmatcheds 6 . Intuitively speaking, such fields will be used in the derived classes to indicate which kind of matching has been obtained (viz., exact, plug-in or unmatched) and (in case of unmatched) which features are left unmatched.

Finally, it is worth noting that the match method is declared abstract. So, (thanks to JAVA inheritance) the derived classes must declare a method which overrides it.

\footnotetext{
${ }^{6}$ Since policies matching only consists in checking whether they are applicable to $\mathrm{n}$, only the result field is defined.
} 


\section{Implementation of the exact matchmaker}

The first matchmaker to implement is the exact one (viz., the "三" of Section 3.1). The source $\operatorname{code}^{7}$ of such a matchmaker is reported in Listings 5.13 and 5.14 .

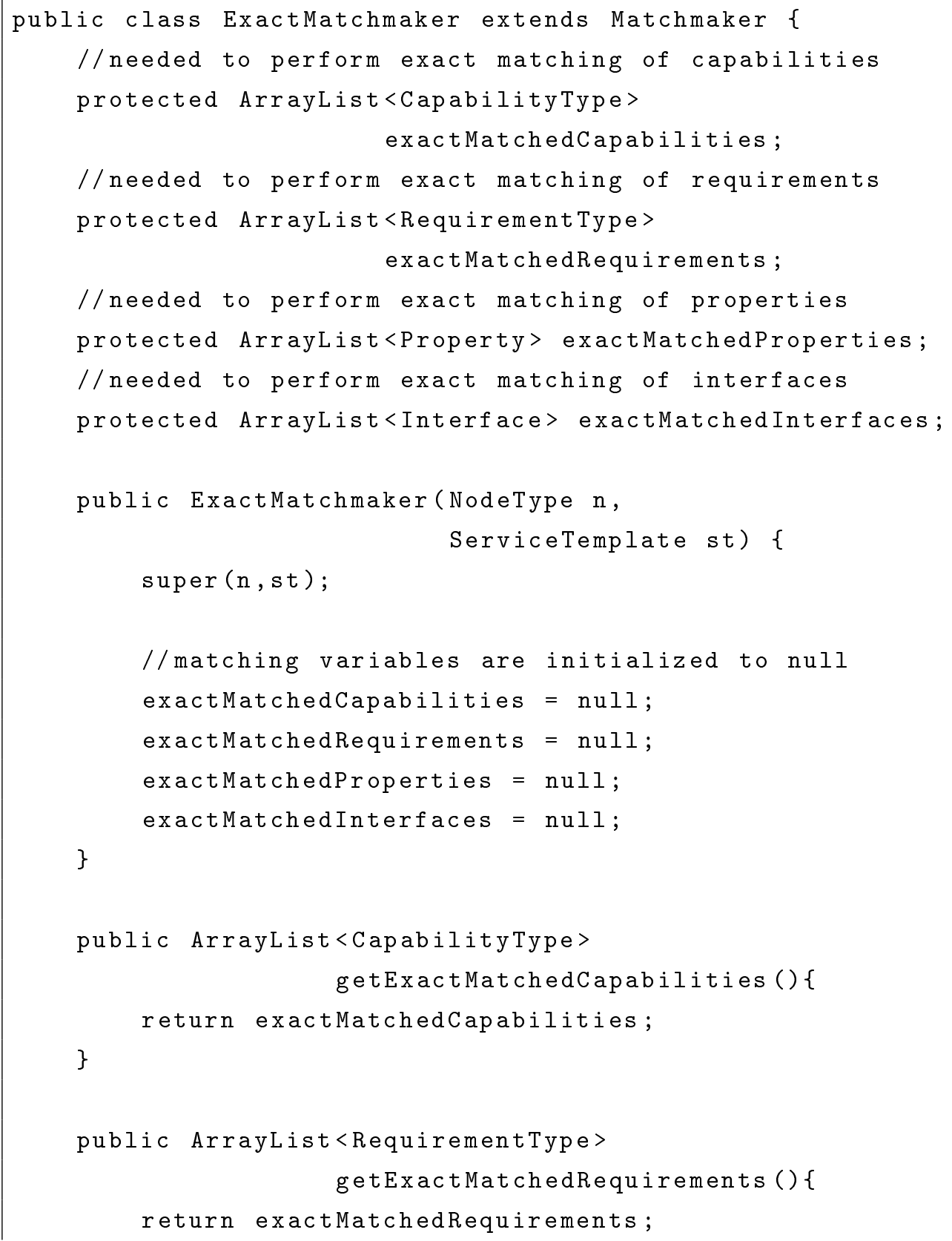

${ }^{7}$ To better understand the unexplained source code please look at inline comments. 


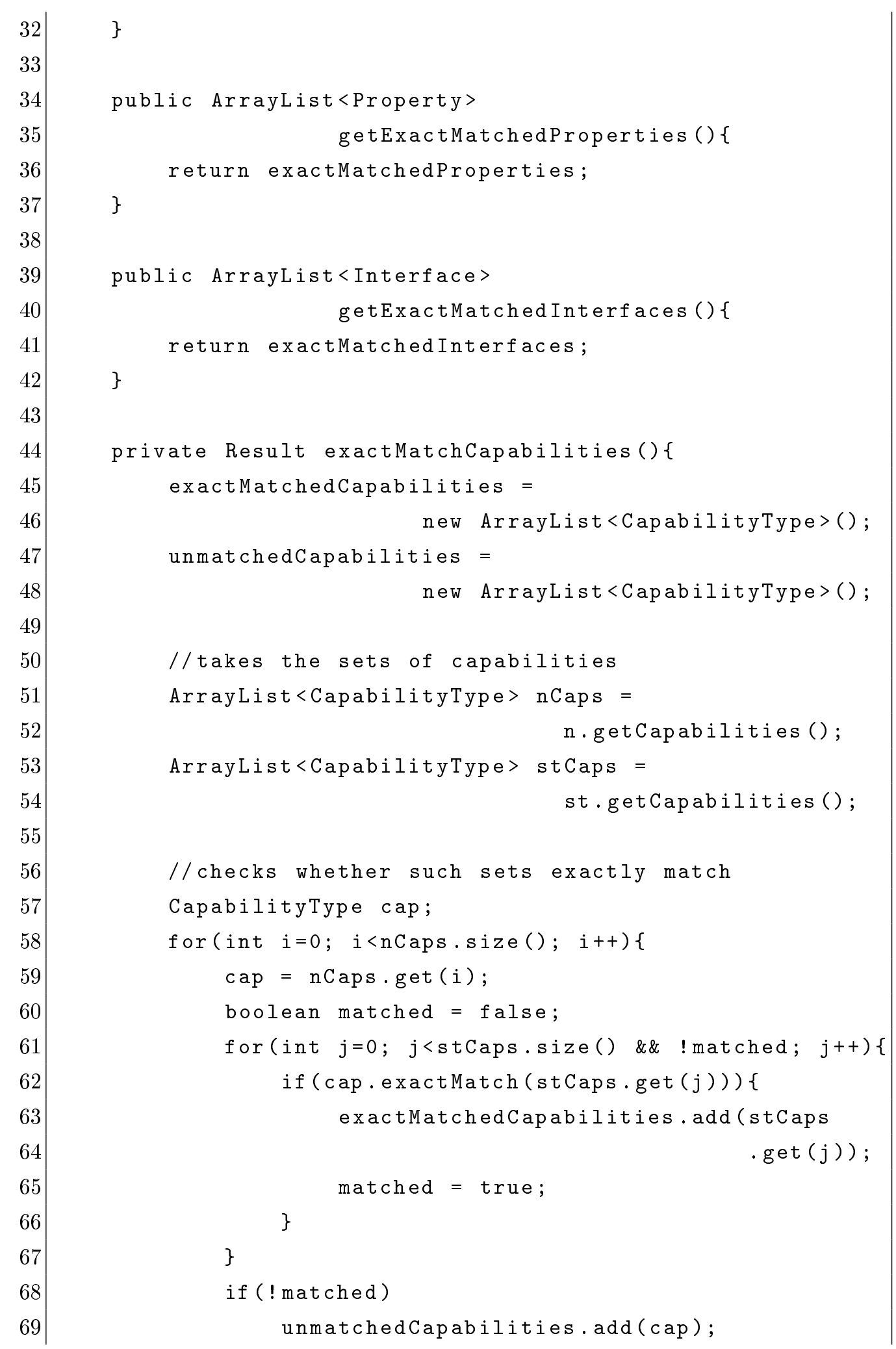




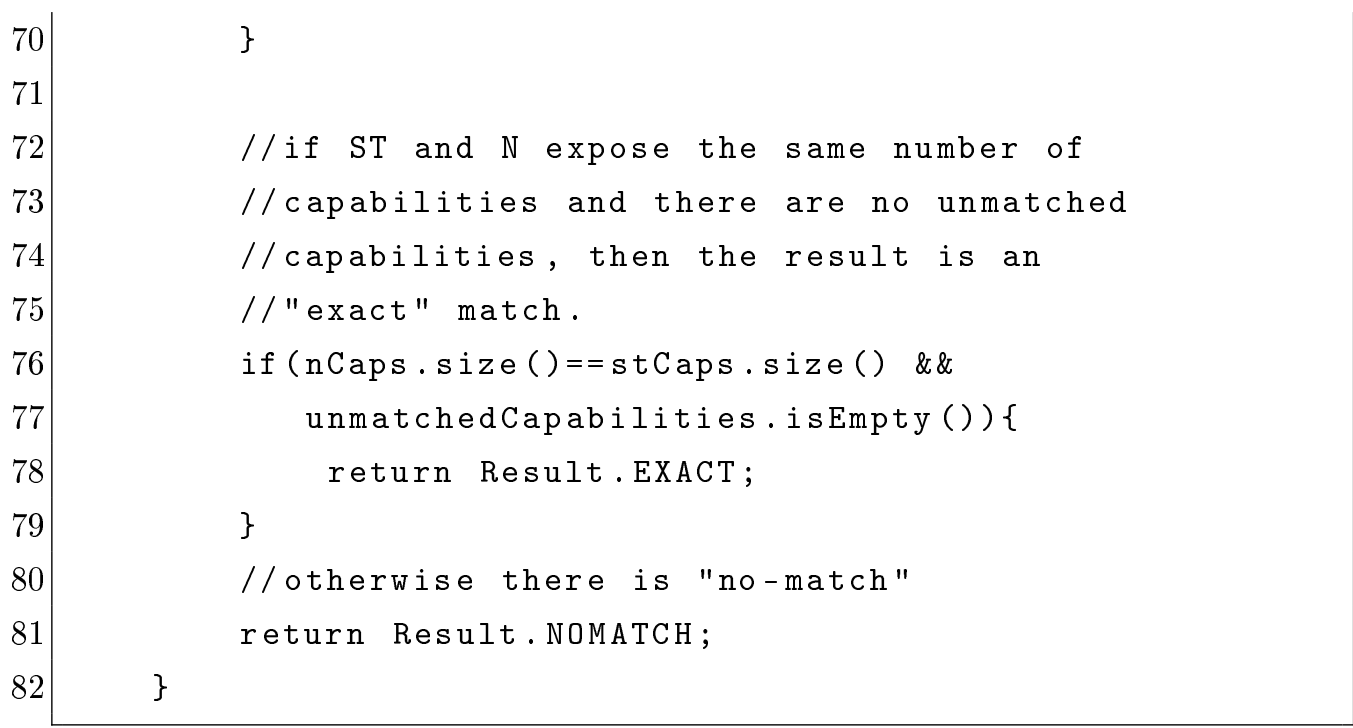

Listing 5.13: JAVA implementation of the ExactMatchmaker (1).

Let us focus on the exactMatchCapabilities method (which returns a Result to indicate whether the capabilities of NodeType $\mathrm{n}$ and those of the ServiceTemplate st exactly match). We know that, intuitively speaking,

\section{n.CapabilityDefinitions $\equiv_{C}$ st.Capabilities}

if and only if the capabilities of $\mathrm{n}$ are in a one-to-one correspondence with those of st. To obtain such a one-to-one correspondence we simply have to check whether

- for each capability cap of $\mathrm{n}$ there exists a capability of st which is in exactMatching with cap (lines 56-70), and

- $\mathrm{n}$ exposes the same number of capabilities as st does (lines 76-77).

If this is the case, then we obtain that $\mathrm{n}$ exactly matches st (line 79). Otherwise, we obtain the unmatched case (line 81).

It is worth noting that, if a capability is matched, then the relative capability of st is stored in the exactMatchedCapabilities set (in order 
to ease st adaptation - lines 62-64). Otherwise, the unmatched capability cap of $\mathrm{n}$ is stored in the unmatchedCapabilities set (lines 68-79).

Please note that, as reported in Listing 5.14 , the requirements, properties and interfaces matching (lines 84-121, 129-164 and 166-201, respectively) is analogous to the one above presented. This is clearly not the case of policies: we know that, intuitively speaking,

\section{Policy Type applicable to $\mathrm{n} \equiv_{P O}$ st.Policies}

consists in checking whether st's policies are of a type applicable to $\mathrm{n}$ (lines 123-127).

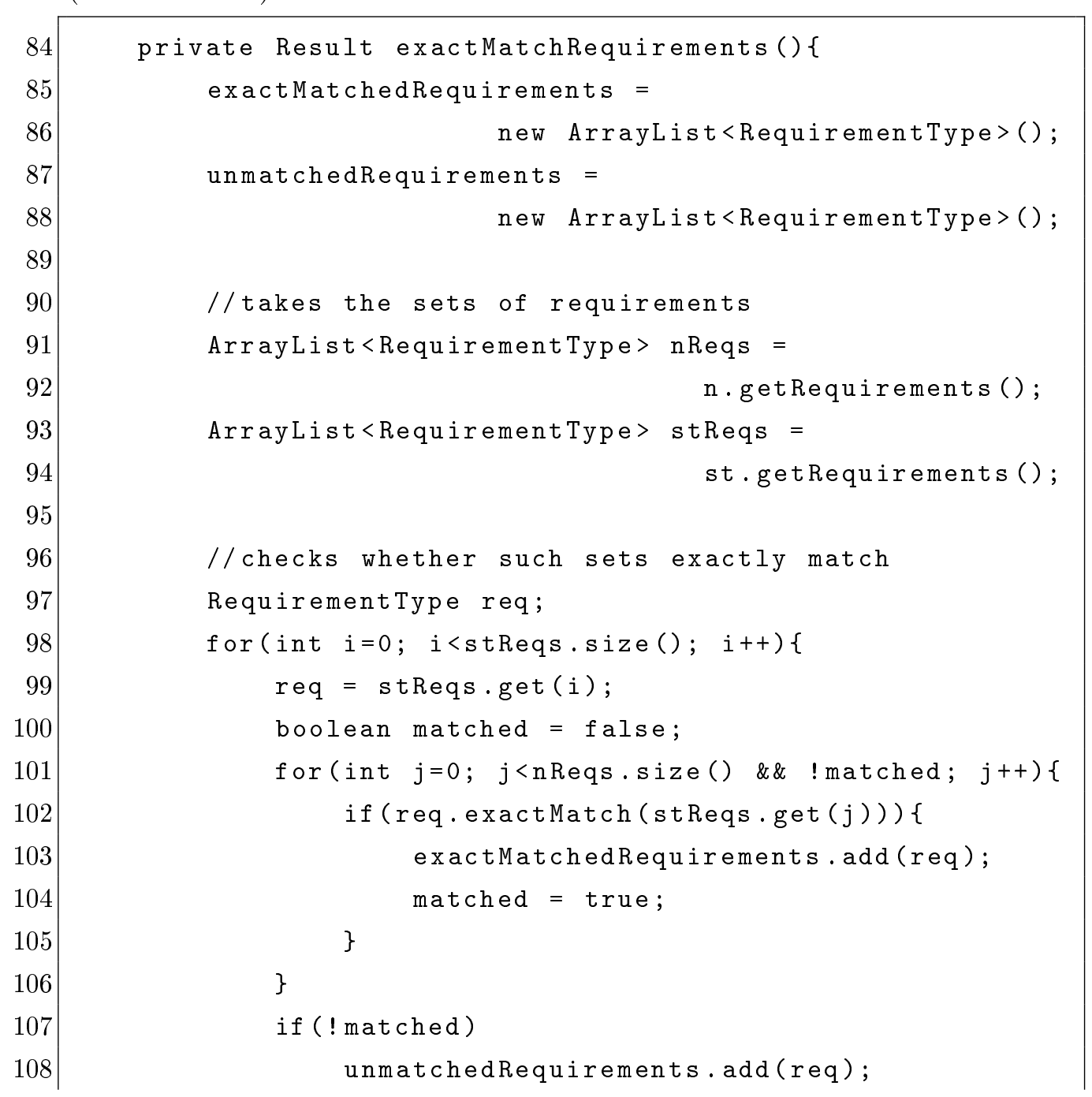




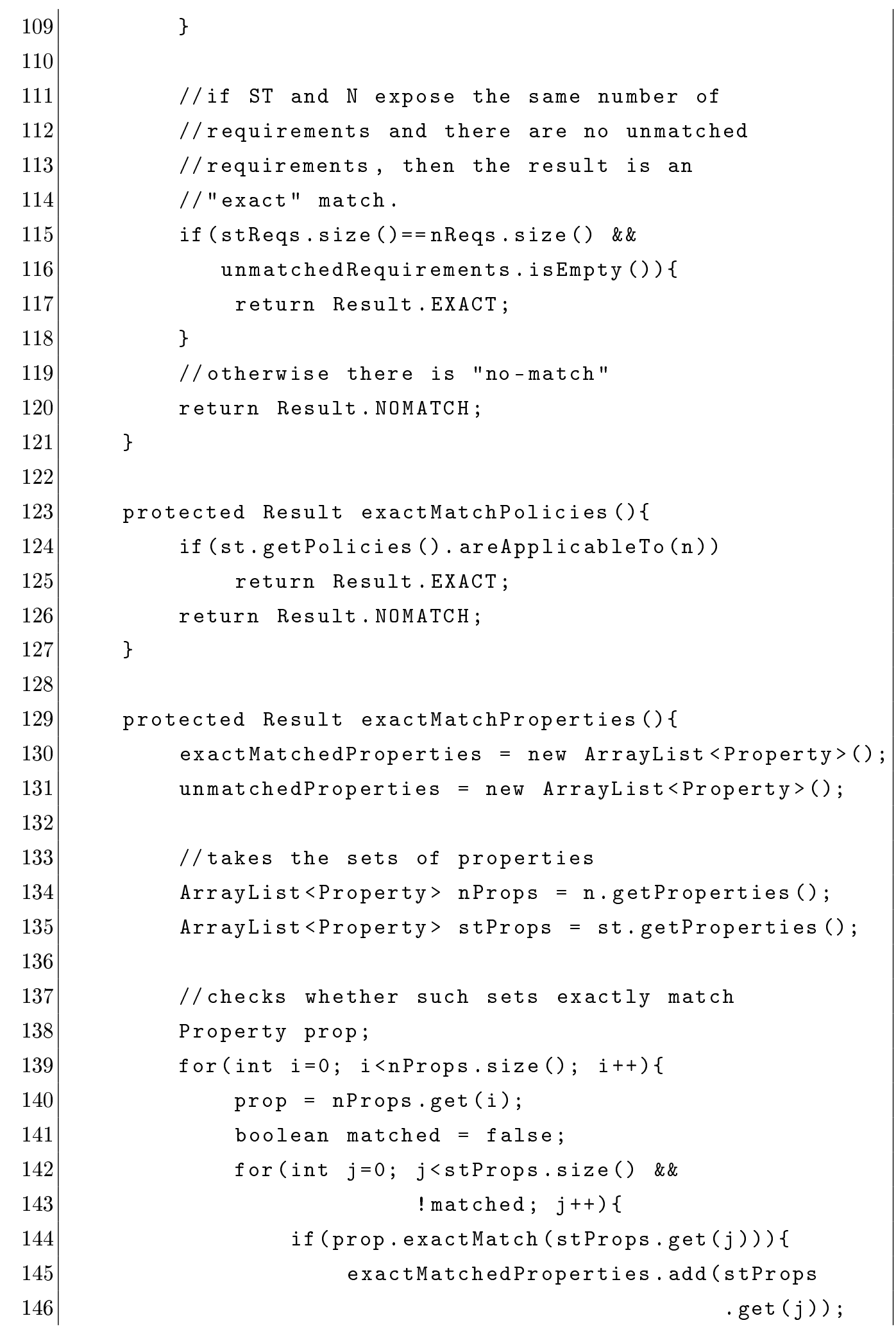




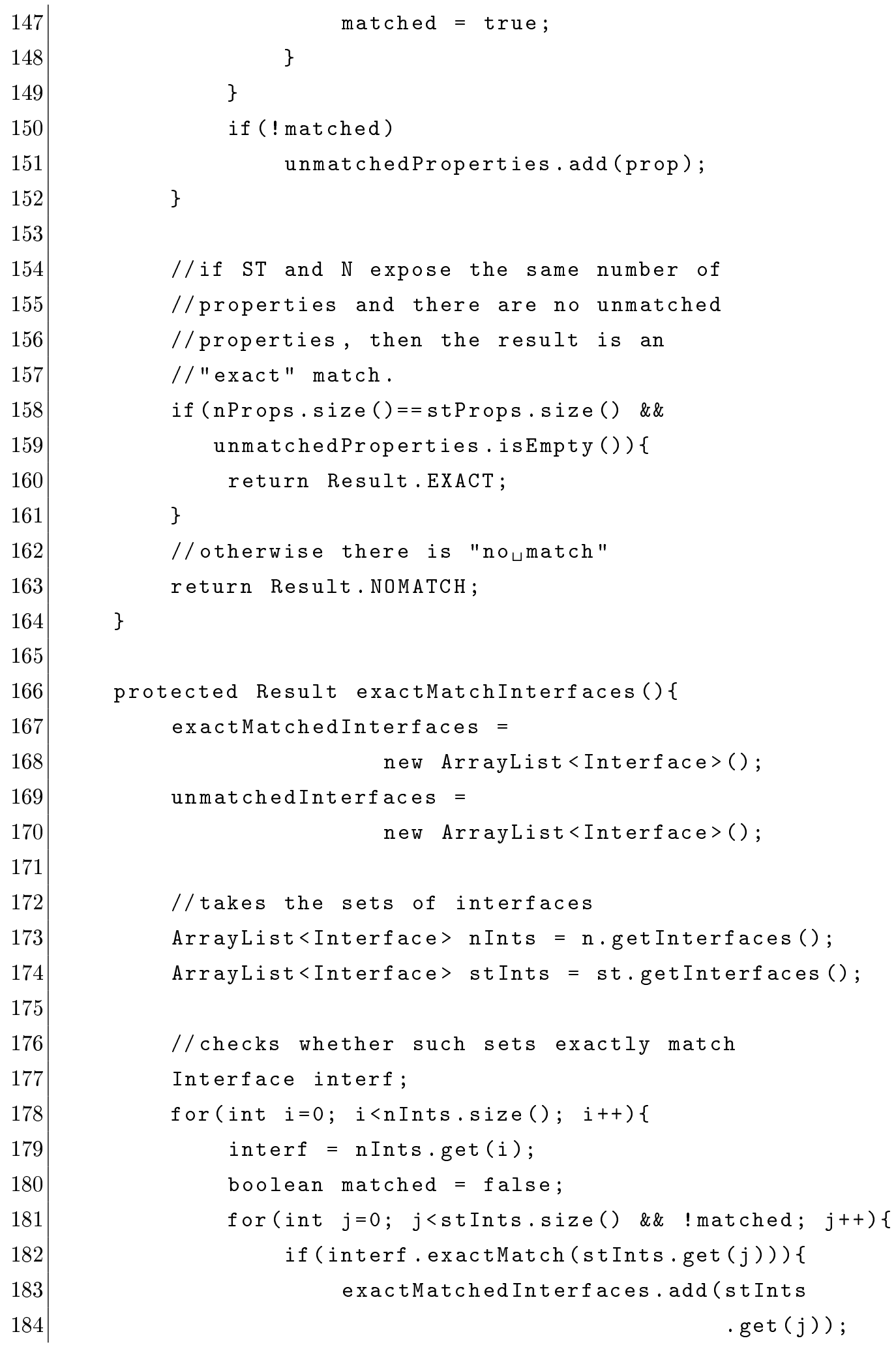




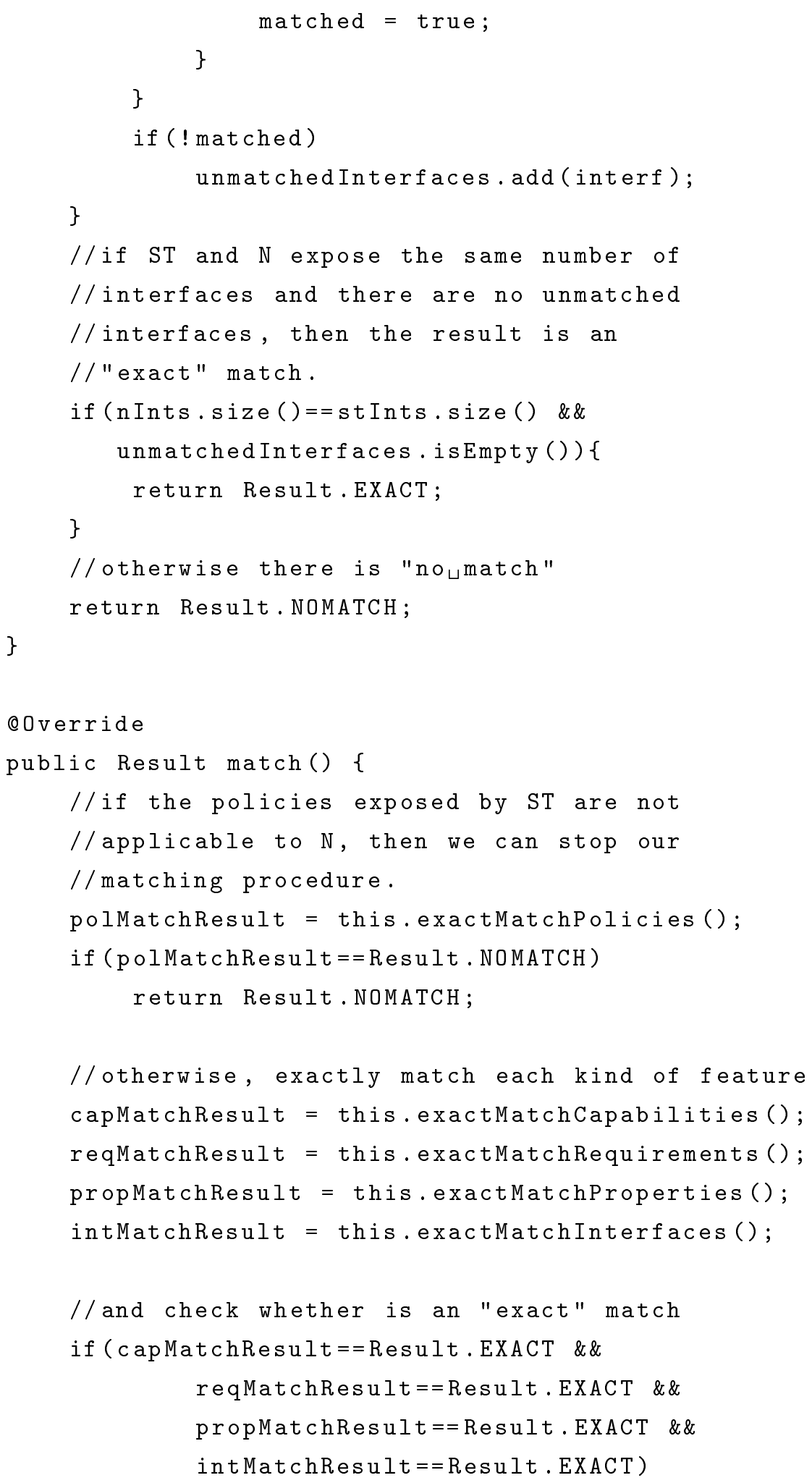




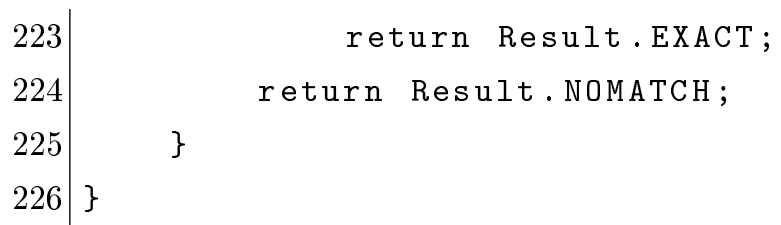

Listing 5.14: JAVA implementation of the ExactMatchmaker (2).

Once the features exact matching has been defined, we can override the match method to implement the

$$
\mathrm{n} \equiv \mathrm{st}
$$

expression. So, after having checked whether st's policies are applicable to $\mathrm{n}$ (lines 205-210), we invoke private methods to check whether the capabilities, requirements, properties and interfaces of $\mathrm{n}$ exactly matches those of st (lines 213-216). Once this has been performed, we can employ partial results to compute the overall matching Result (lines 219-224).

\section{Implementation of the plug-in matchmaker}

So far, we developed the exact matchmaker. Now, we want to extend it in order to obtain the plug-in one (Listings 5.15 and 5.16).

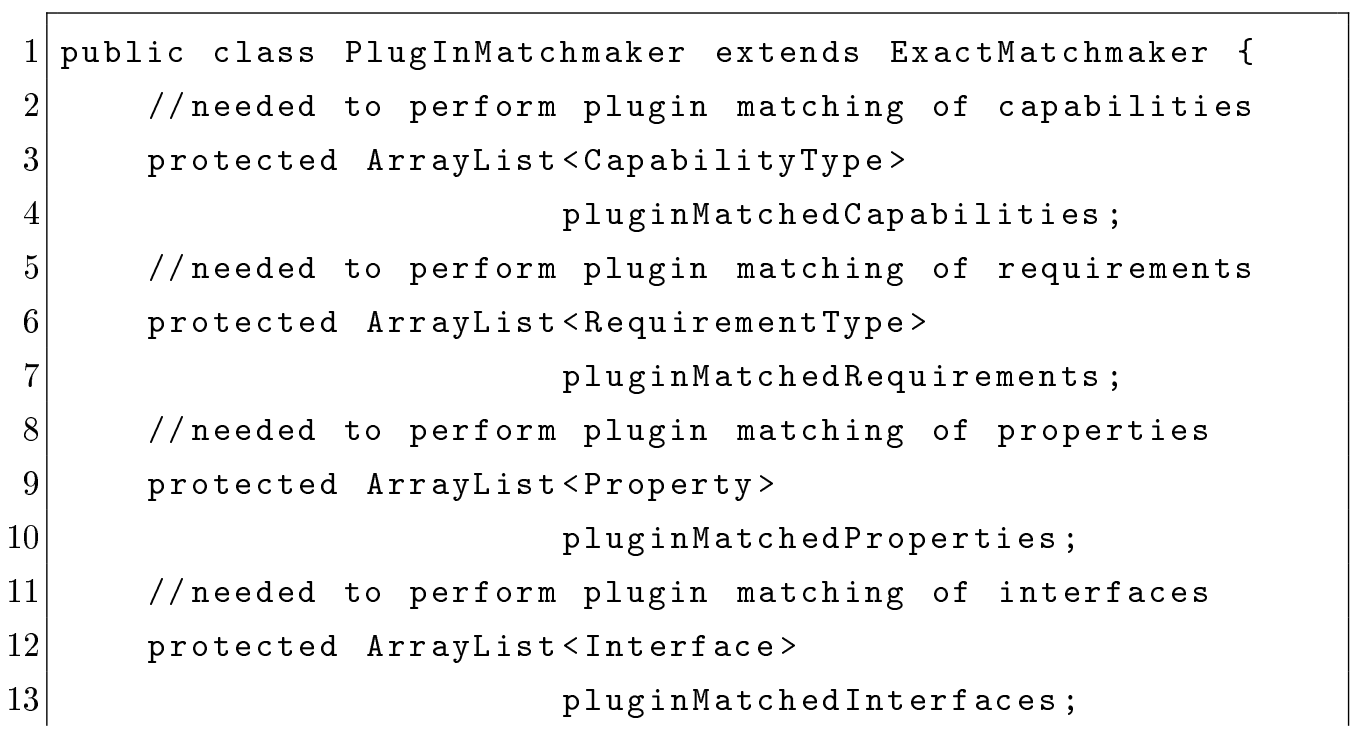




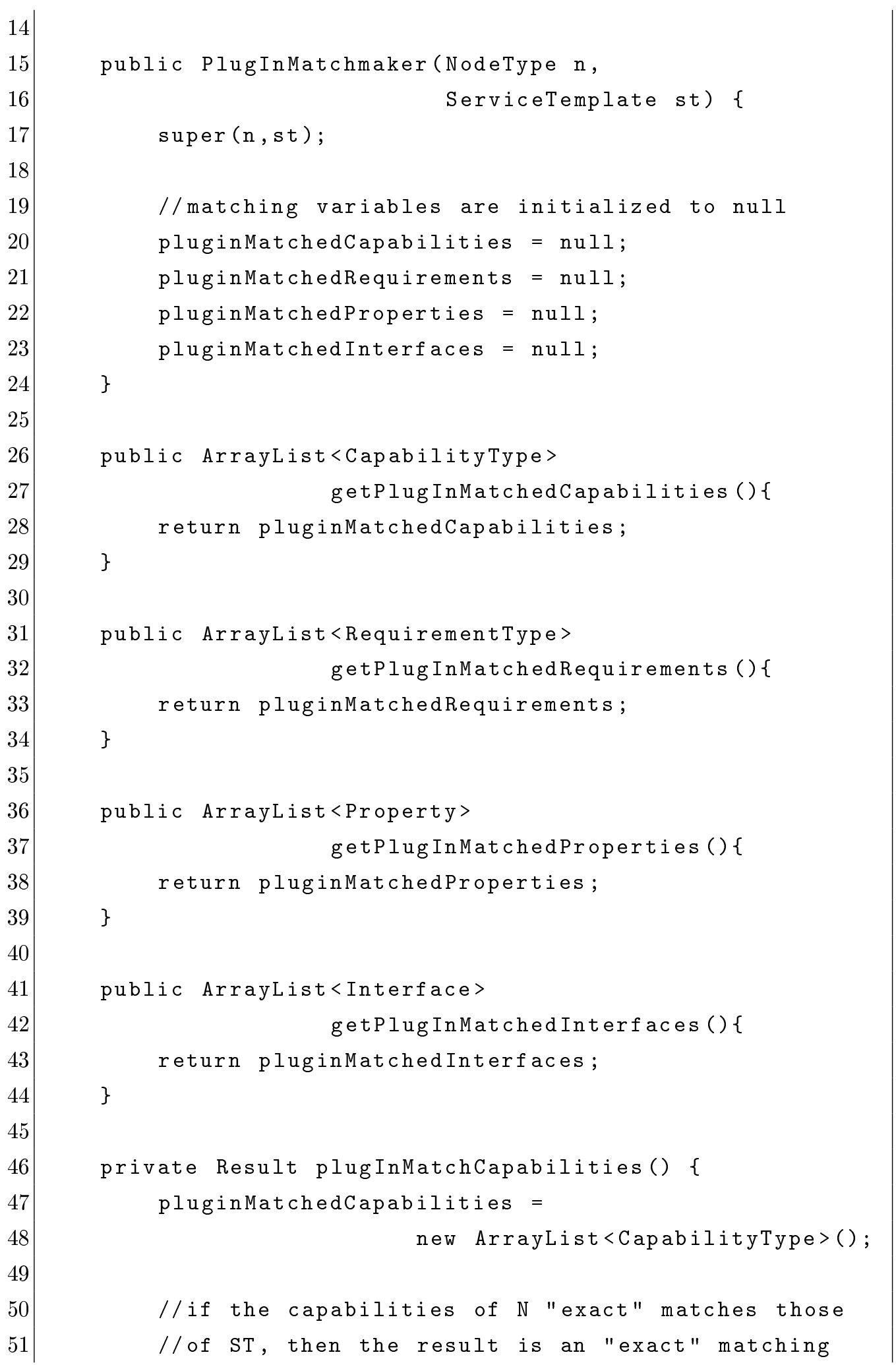




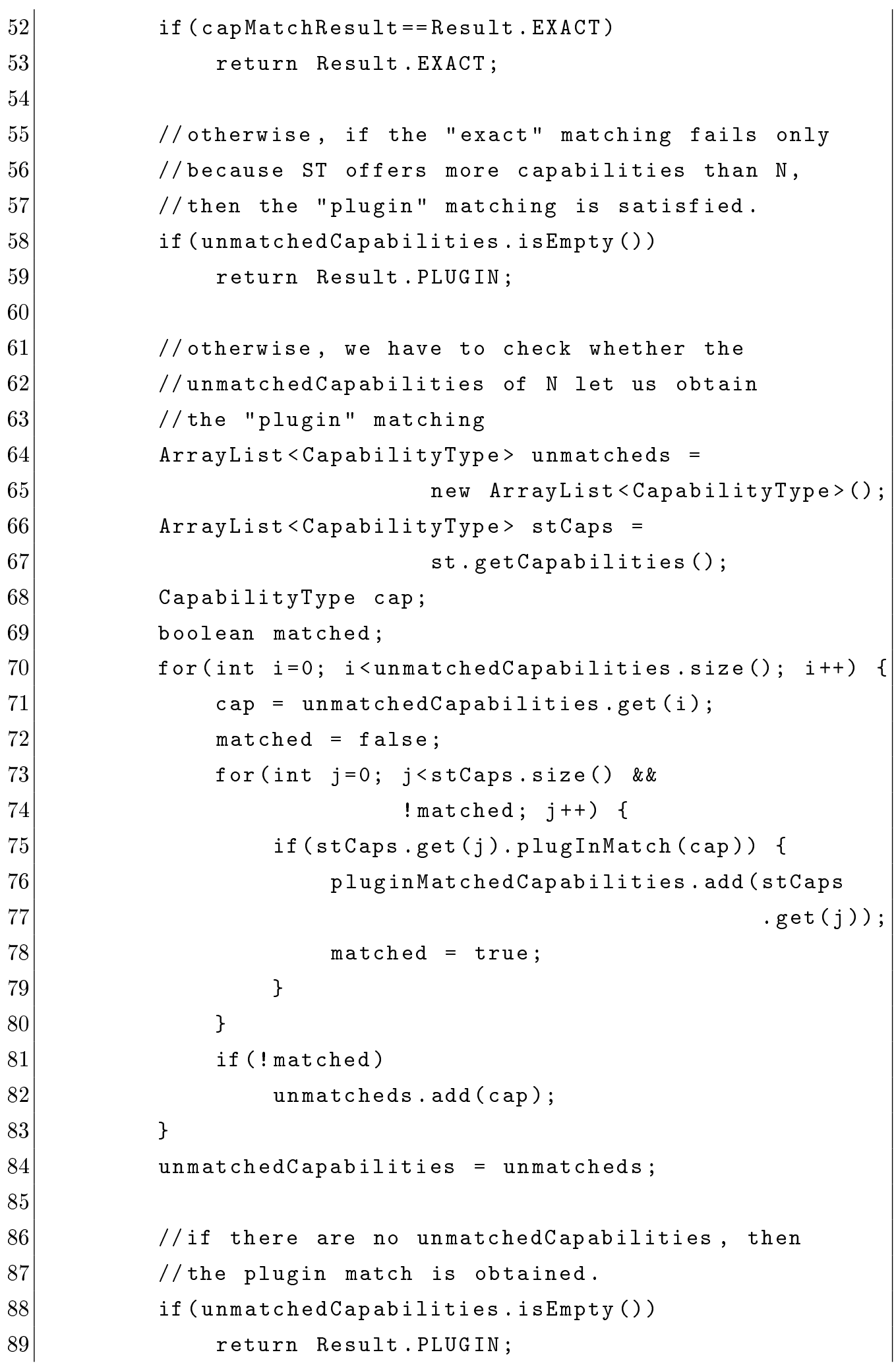




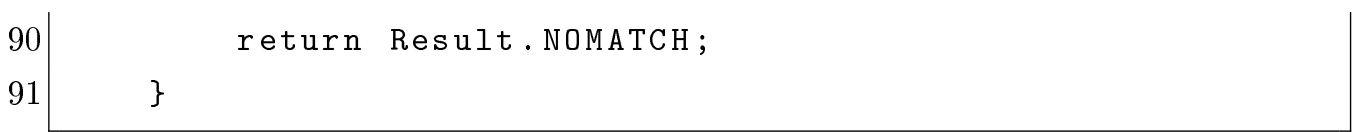

Listing 5.15: JAVA implementation of the PlugInMatchmaker (1).

Let us focus on the plugInMatchCapabilities method (which returns a Result to indicate whether the capabilities of the NodeType $\mathrm{n}$ plugin match those of the ServiceTemplate st). We know that, intuitively speaking,

\section{n.CapabilityDefinitions $\subseteq_{C}$ st.Capabilities}

if and only if for each capability cap of $\mathrm{n}$ there exists a capability of $\mathrm{st}$ which is in plugInMatching with cap (lines 55-84). If this is the case, then we obtain that $\mathrm{n}$ exactly matches st (lines 86-89). Otherwise, we obtain the unmatched case (line 90). Please note that, if the capabilities of $n$ exactly match those of st, then there is no need to proceed with the plug-in matching (lines 50-53).

As before, the way in which requirements, properties and interfaces matching is implemented (lines 93-136, 138-182 and 184-224) is analogous to the capabilities one (Listing 5.16).

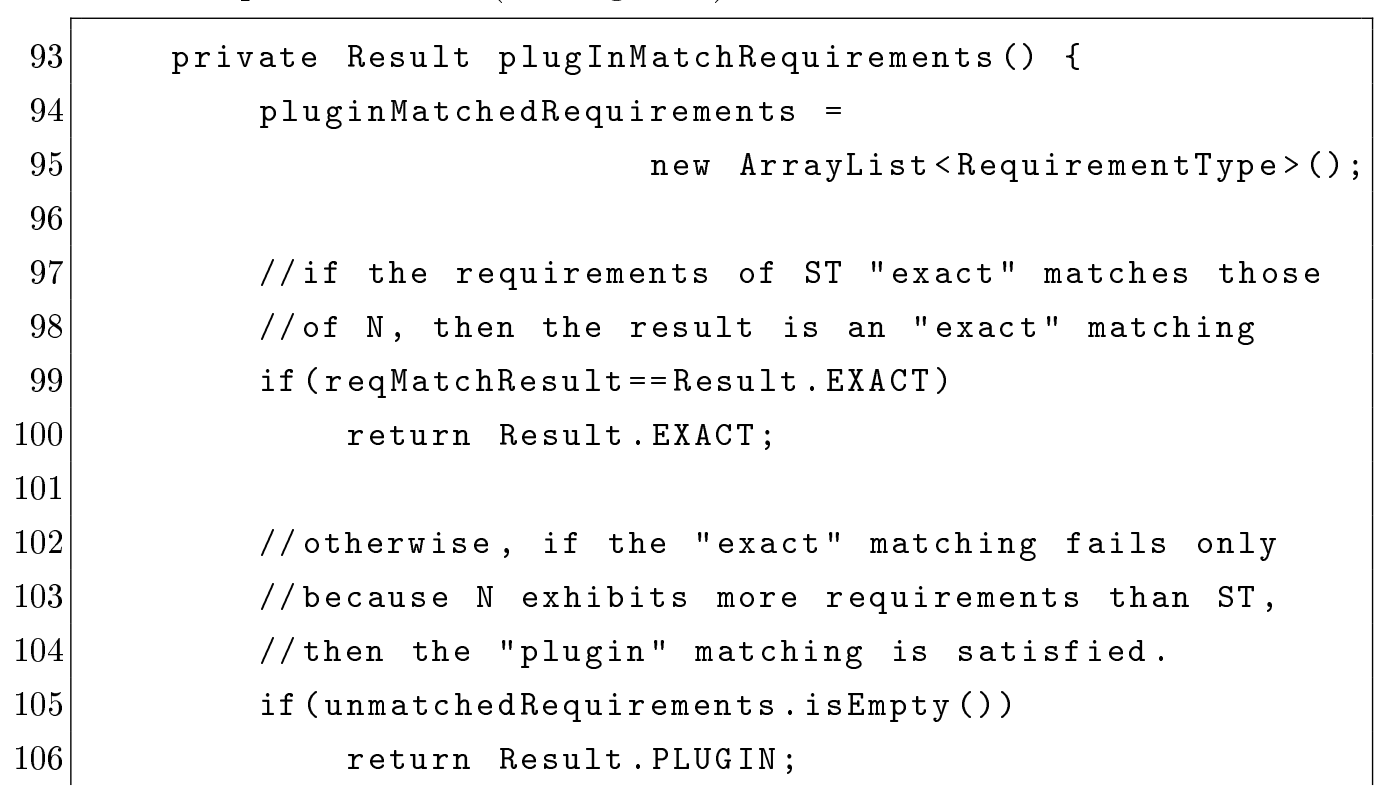




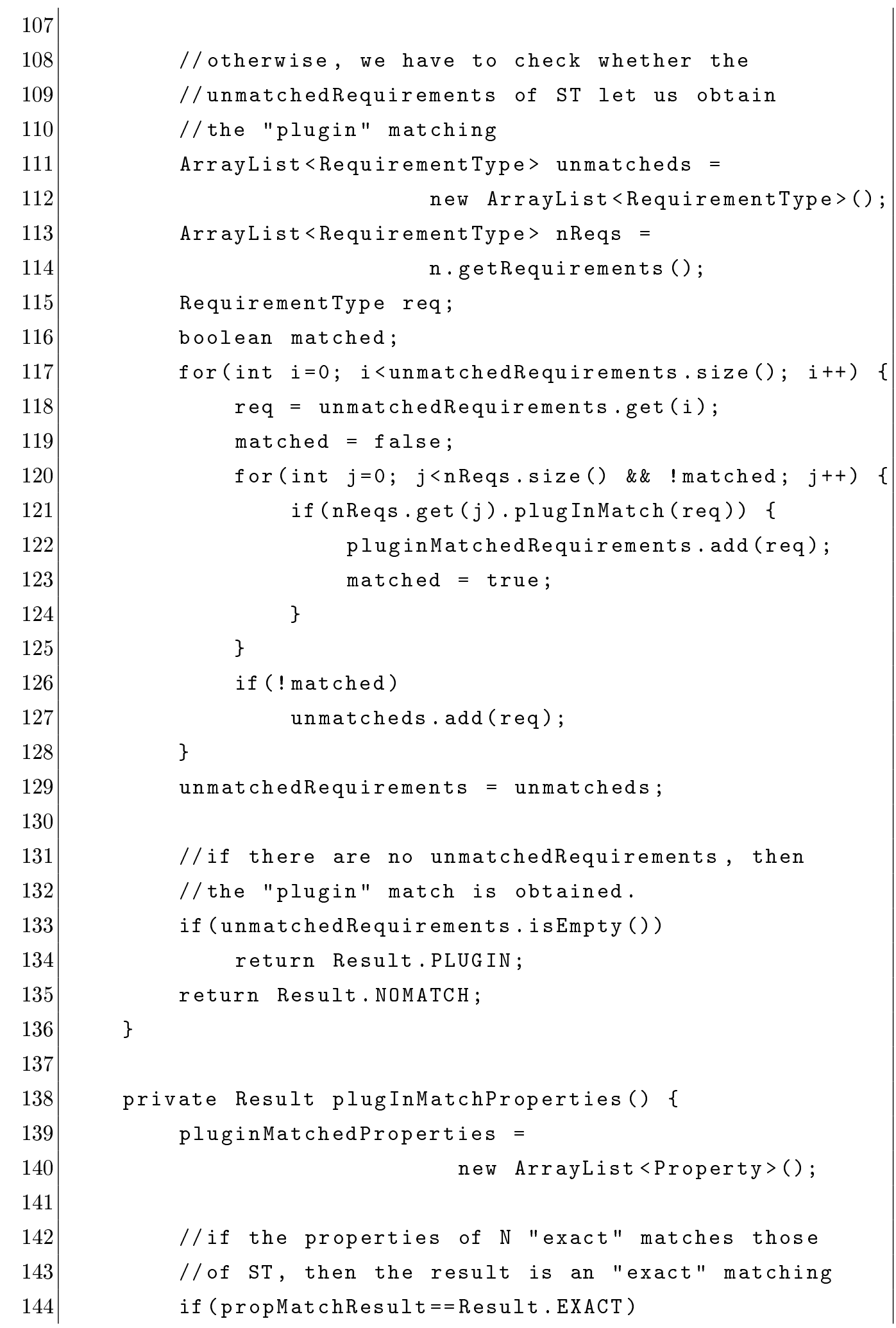




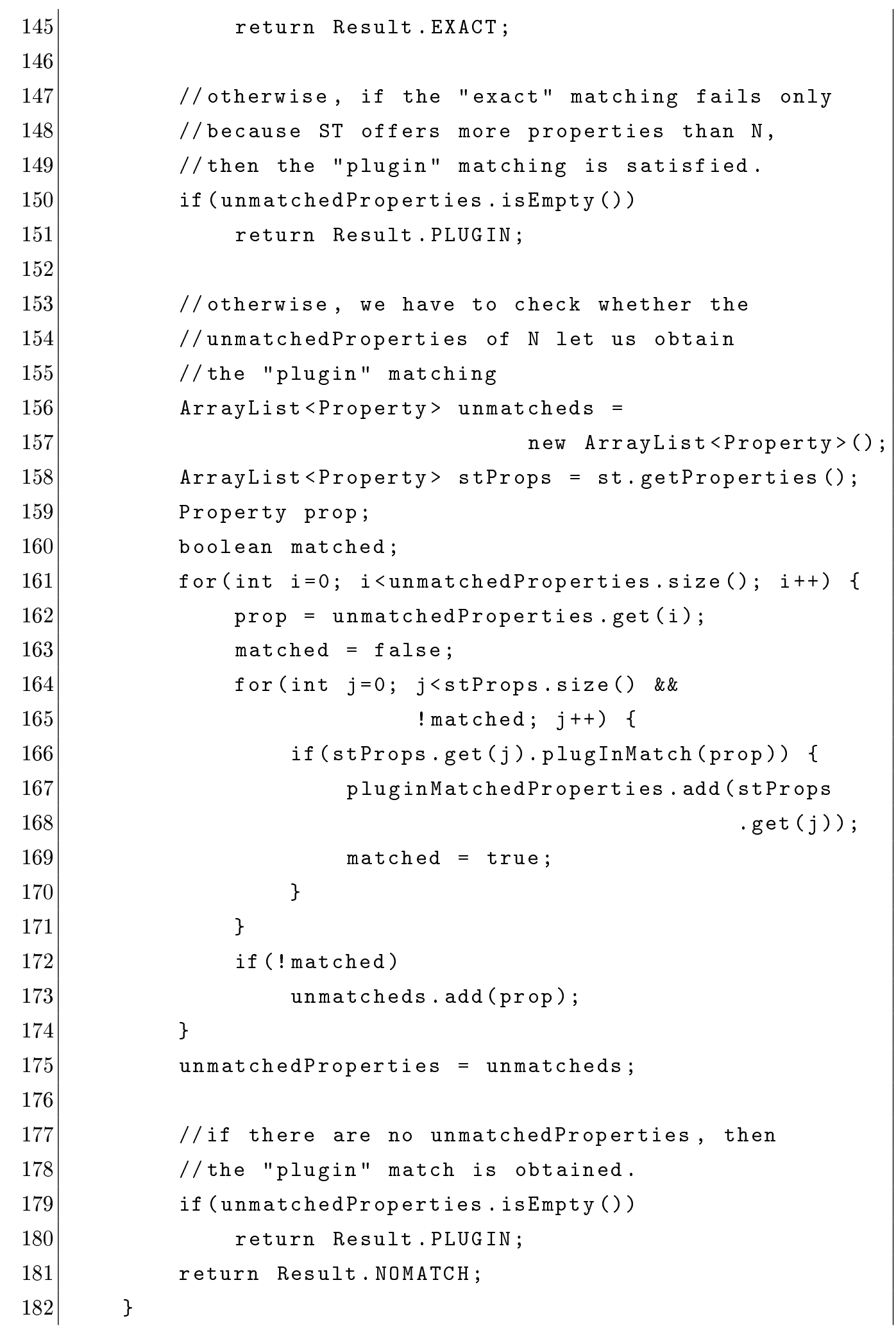




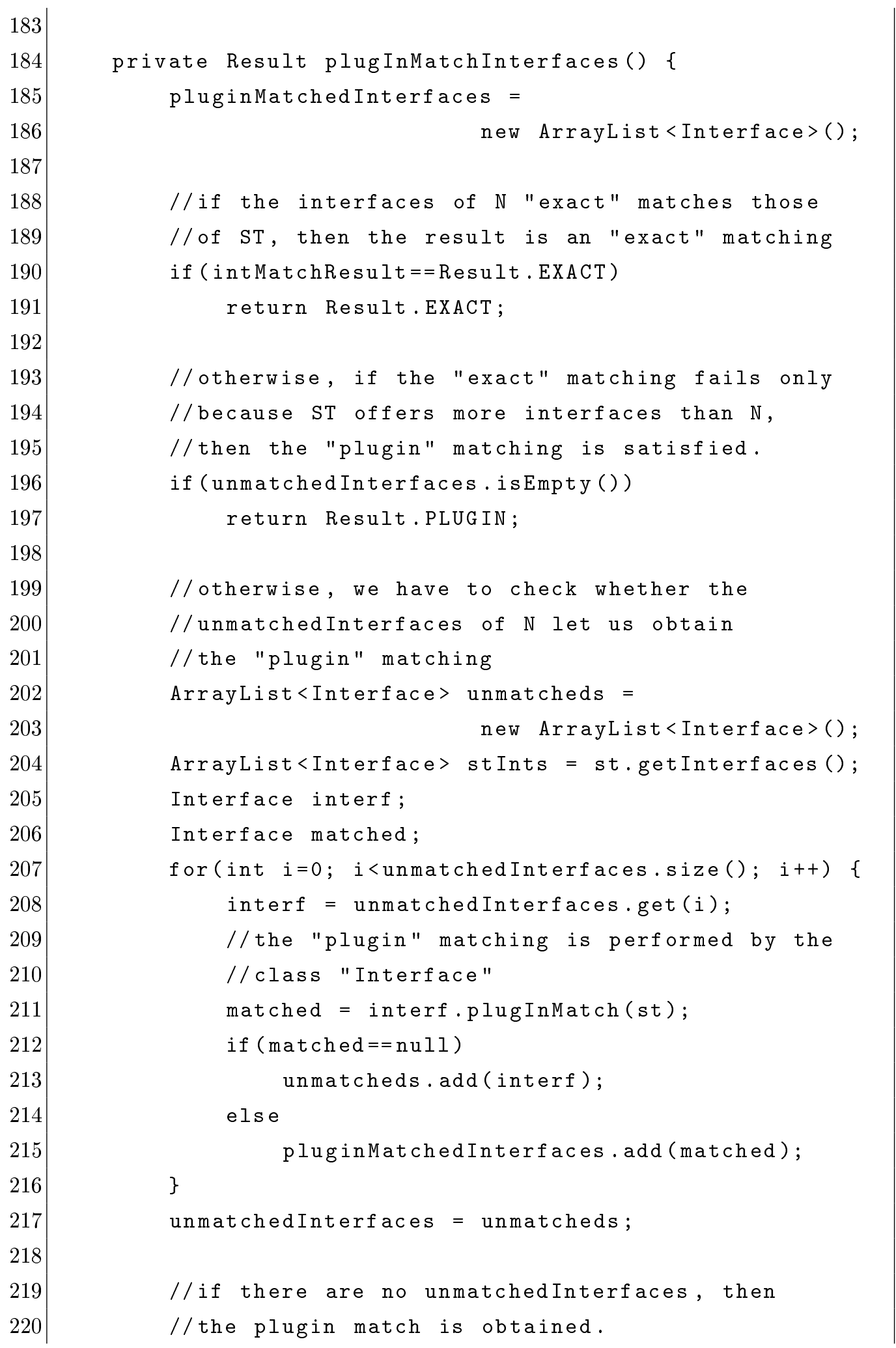




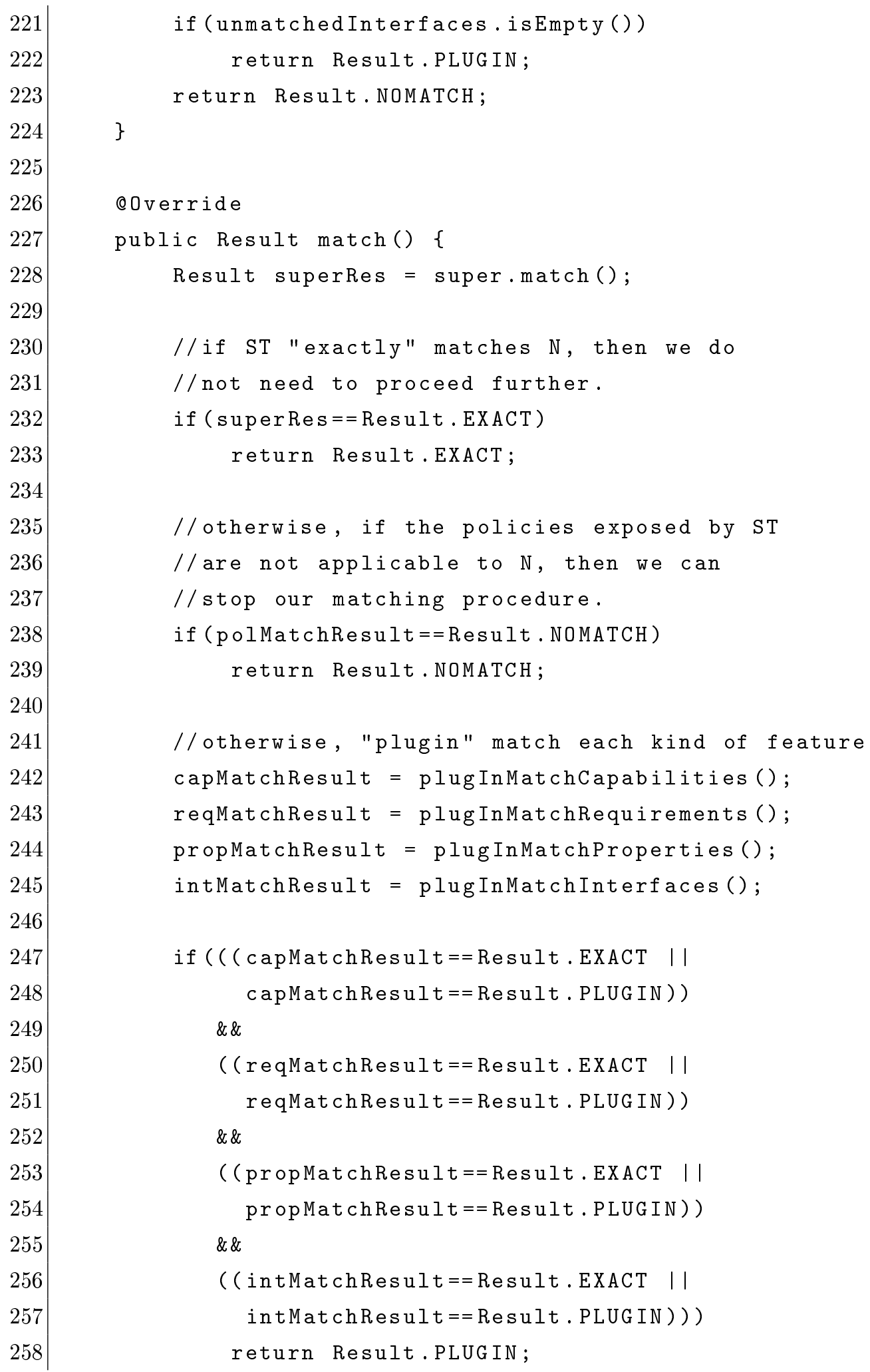




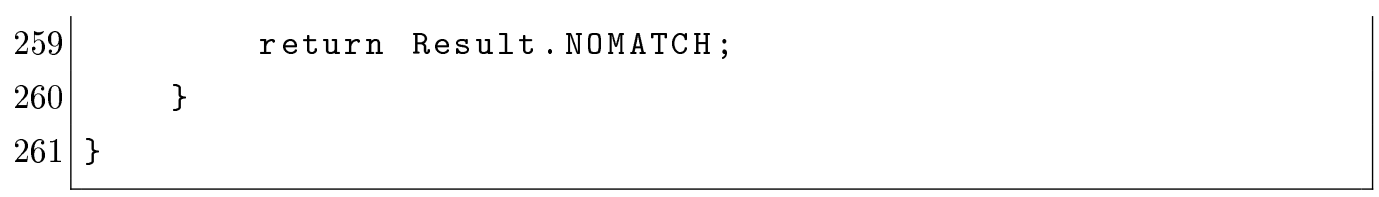

Listing 5.16: JAVA implementation of the PlugInMatchmaker (2).

Once the features plug-in matching has been defined, we can override the match method to implement the

$$
\mathrm{n} \subseteq \mathrm{st}
$$

expression. So, after having checked whether $\mathrm{n}$ exact matches st (lines 230-233) and whether st's policies are applicable to n (lines 235-239), we invoke private methods to check whether the capabilities, requirements, properties and interfaces of $\mathrm{n}$ plug-in matches those of st (lines 242-245). Once this has been performed, we can employ partial results to compute the overall matching Result (lines 247-259).

\subsection{Concluding remarks}

So far, we provide a (tested 8 ) JAVA implementation of the black-box exact and plug-in matching notions. It is worth noting that the computed matching sets (such as exactMatchedCapabilities, pluginMatchedCapabilities and unmatchedCapabilities) will be employed in the development of the st adaptation and of the other matching notions. How to do it is out of the purposes of this chapter (since we only want to demonstrate the feasibility of the proposed matching procedure).

\footnotetext{
${ }^{8}$ Appendix $\mathrm{A}$ shows an example of made tests.
} 


\section{Chapter 6}

\section{Conclusions}

Before concluding, it is worth making some final remarks so as to:

- summarize the contributions of this thesis to the TOSCA specification (Section 6.1),

- discuss related work (Section 6.2), and

- provide an overview of possible future work (Section 6.3).

\subsection{Summary of contributions}

In this thesis, after defining the notion of exact matching between TOSCA ServiceTemplate and NodeType elements, we have defined three other types of matching (plug-in, flexible and white-box), each permitting to ignore larger sets of non-relevant syntactic differences when type-checking ServiceTemplate elements with respect to node types. More precisely:

- the plug-in matching extends the exact one by considering a ServiceTemplate that "require less" and "offers more" than a NodeType compatible with the latter; 
- the flexible matching in turn extends the plug-in one by employing ontologies to check whether differently named features are semantically equivalent (so as to ignore non-relevant syntactic differences);

- the white-box matching in turn extends the flexible one by searching missing (equivalent) features inside the service topology. It still employs ontologies to check whether differently named features can be considered semantically equivalent. Furthermore, it employs a recursive algorithm to detect available compositions of operations which are semantically equivalent to needed (missing) operations.

Furthermore, we have also described how a ServiceTemplate that plug-in, flexibly or white-box matches a NodeType can be suitably adapted so as to exactly match it.

As we already mentioned at the very beginning of this work, the presented results intend to contribute to the formal definition of TOSCA. More precisely, the different types of matching defined in this thesis can be used to develop a matchmaker to be fruitfully integrated in the TOSCA implementations that are currently under development (such as the Valesca editor [32] and the OpenTOSCA IDE [27]) in order to enhance their typed node matching capabilities. The development of such (pluggable) matchmaker will contribute to cloud service portability and multi-cloud service development. Indeed, with the availability of such an implementation, a cloud service developer will have the possibility to:

- employ more available (adapted) cloud services instead of developing her application's encompassed components,

- migrate more application's components across heterogeneous clouds by changing the used available (adapted) cloud services, and

- choose between more different cloud service providers the one which provides the compatible service with the best quality-price ratio. 


\subsection{Related work}

\section{Service matching}

As we already mentioned at the very beginning of this thesis, our work started from the observation that while the matching between ServiceTemplate and NodeType elements is indicated in [26] as a way to instantiate TOSCA NodeType elements, no (formal) definition of matching is given either in 25] or in [26]. A concrete definition of matching for TOSCA is used in [33] to define a way to merge TOSCA services by matching entire portions of their topology templates. The definition of matching of single service components employed in [33] is however very strict, as two service components are considered to match only if they expose the same qualified name. This work aims at contributing to the TOSCA specification by proposing four definitions of matching between ServiceTemplate and NodeType elements, each identifying larger sets of ServiceTemplate elements that can be adapted so as to (exactly) match a NodeType.

The problem of how to match (Web) services has been extensively studied in recent years. Many approaches are ontology-aware [28], like for instance the ontology-aware matchmaker for OWL-S services described in [16]. Other approaches are behaviour-aware, like the (ontology-aware) trace-based matching of YAWL services defined in [9], the (ontologyaware) behavioural congruence for OWL-S services defined in [3], or the graph transformation based matching defined in [12] and the heuristic black-box matching described in [14] for WS-BPEL processes. The main difference between the aforementioned approaches and ours is the type of information considered when matching single nodes. The matching levels considered for instance in [16] and [14] are all defined in terms of input and output data, while we consider also technology requirements and capabilities, properties and policies.

On the other hand, many proposals of QoS-aware service matching 
have been developed, like for instance [19] or [23]. Generally speaking, the notion of matching defined in the present thesis differs from most QoSaware matching approaches since it compares types rather than actual values of extra-functional features (like QoS). A type-based definition of matching is defined in [13] to type check stream flows for interactive distributed multimedia applications. While the context of [13] is different from ours, two of the matching conditions considered in [13] resemble our notions of exact and plug-in matching, even if for simpler service abstractions.

Summing up, to the best of our knowledge, our definition of matching is the first definition of (TOSCA) node matching to take into account both functional and extra-functional features, by relying both on types and on ontologies to overcome non-relevant syntactic information.

\section{Service adaptation}

As we already mentioned (in Sections 3.2 and 3.3) a ServiceTemplate ST that plug-in or flexibly matches a NodeType $N$ can be adapted into a new Service Template $S T^{\prime}$ that exactly matches $N$. The adaptation technique basically consists of creating a new ServiceTemplate $S T^{\prime}$ that includes $S T$ as internal node, and of suitably exposing (via BoundaryDefinitions) the capabilities, policies, properties, and interfaces of the NodeType to be matched. The transformation implemented by such adaptation vaguely reminds the adaptation techniques described in [9] and [10] to implement (more complex) input-output and behaviour transformations of YAWL workflows, respectively.

Furthermore, as we discussed in Chapter 4 , the adaptation needed to transform a ServiceTemplate ST that white-box matches a NodeType $N$ into a new ServiceTemplate $S T^{\prime}$ that exactly matches $N$ requires to generate a plan to combine a set of operations into an input-output behaviour equivalent to a given operation. As seen, such plans can be generated by 
adapting the ontology-aware discovery algorithm presented in [5].

\subsection{Future work}

The whole thesis orbits on the definition of matchmaking procedures between NodeType and ServiceTemplate elements. A (tested) partial implementation of such matchmaking procedures is described in Chapter 5. Completing the implementation of the whole matchmaker is one direction for immediate future work.

Furthermore, we employed plan generation only when white-box matching the operations of node types and service templates. As someone could note, such a plan generation should be employed also when we (black-box) plug-in/flexibly match such features. Suitably integrating the OperationSETsDiscovering algorithm with such matching procedures is another extension left for future work.

Finally, we restricted our work to service type-checking. It is clear that a full-fledged matchmaker will need to employ also values when comparing services. To do it, we have to move the focus from NodeType elements to NodeTemplate ones (see Figure 6.1). Since the type of NodeTemplate elements is known, we do not encounter problems when type-checking it with respect to service templates. But, how can we match values? To consider more than trivial equality comparison, we have to define a way to indicate how to match those features. As an example, we could think of some kind of (policies/properties) "contracts" to indicate the way in which each single feature value should be matched. A definition of such contracts is another possible extension of this work.

\footnotetext{
${ }^{1}$ Please recall that the objective of such an implementation objective is only to show the JAVA feasibility of the matchmaker.
} 


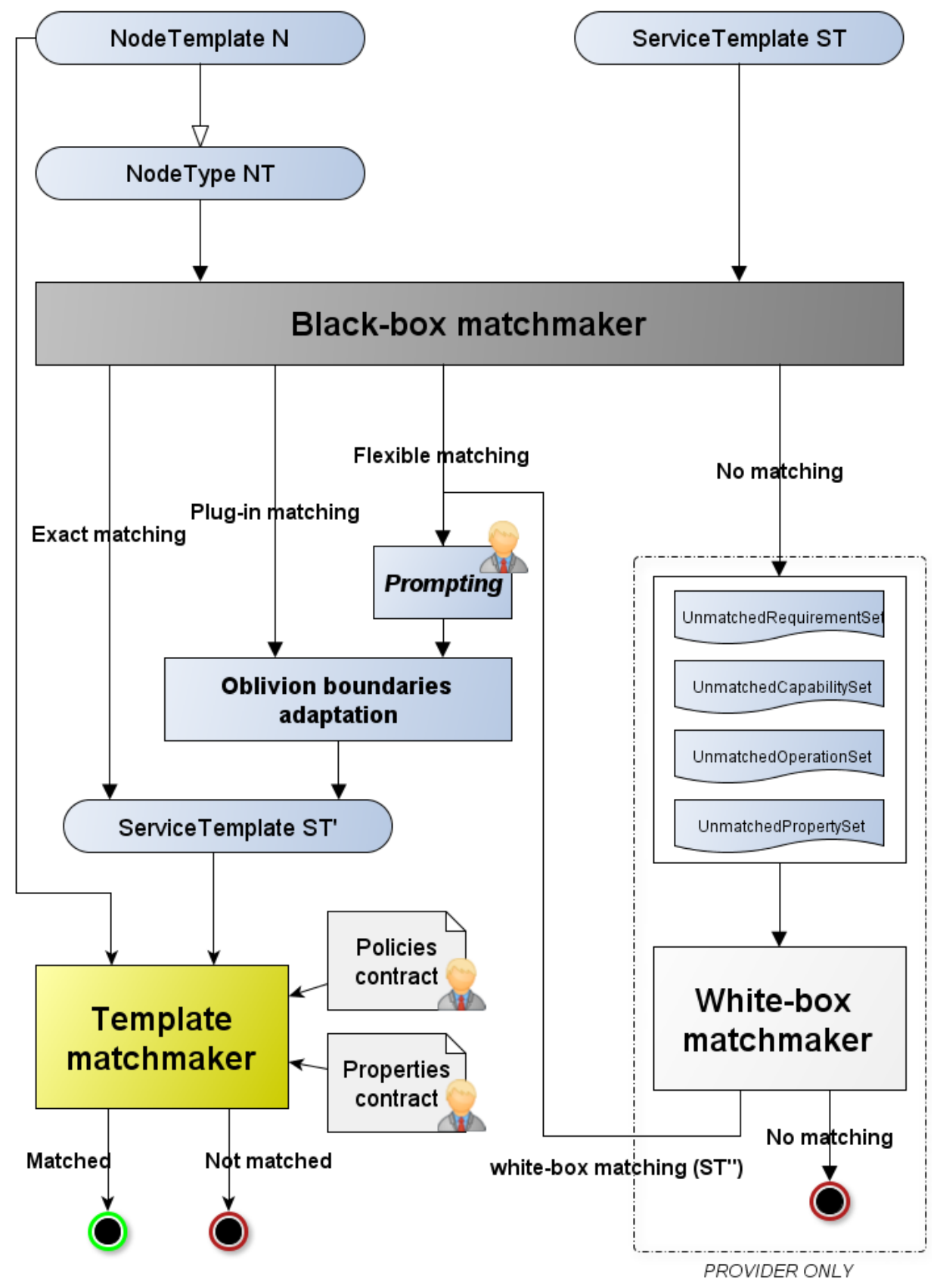

Figure 6.1: Complete matching (and adaptation) procedure. 


\section{Appendix A}

\section{Example of test of the proof-of-concept implementation}

Chapter 5 has shown a (tested) partial implementation of the matchmaking procedure. This Appendix aims at showing an example of the tests which can be executed on such an implementation.

Consider the node type and service templates in Figure A.1 and suppose that all services exhibit a policy (of type RapidCalculatorPolicyType) which is applicable to CalculatorNodeType. Looking at the definitions in Chapter 3, we observe that the following conditions hold:

$$
\begin{gathered}
\text { CalculatorNodeType } \equiv \text { Service, } \\
\text { CalculatorNodeType } \not \equiv \text { ServiceBis, } \\
\text { CalculatorNodeType } \subseteq \text { ServiceBis, and } \\
\text { CalculatorNodeType } \nsubseteq \text { ServiceTer. }
\end{gathered}
$$

We are going to show that, employing the developed (partial) matchmaker, we obtain such results. 


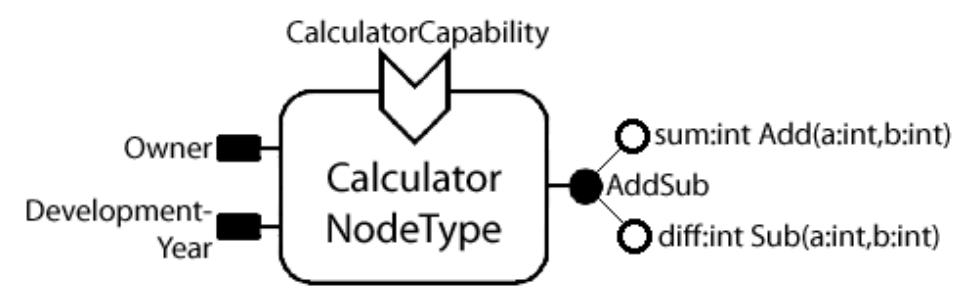

(a)

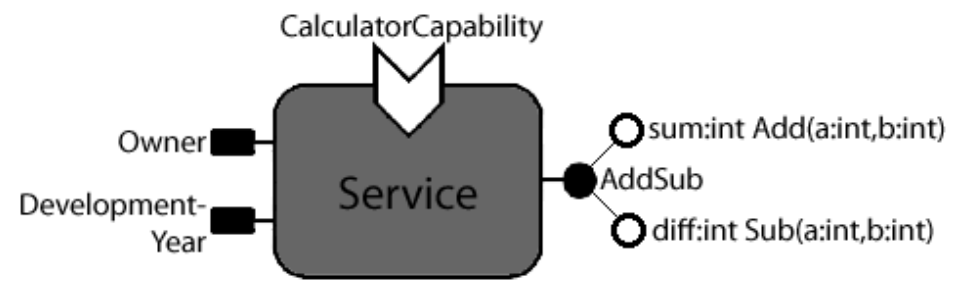

(b)

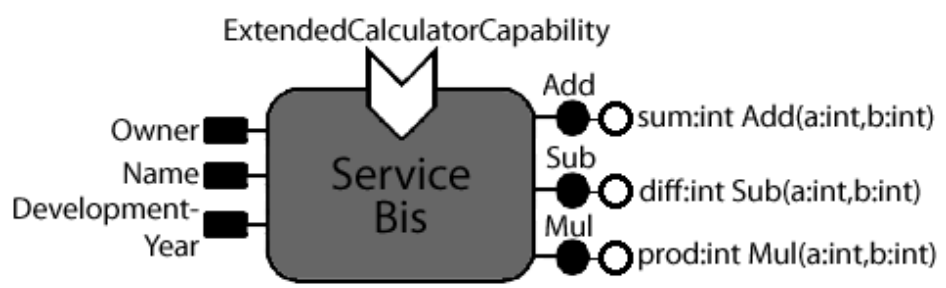

(c)

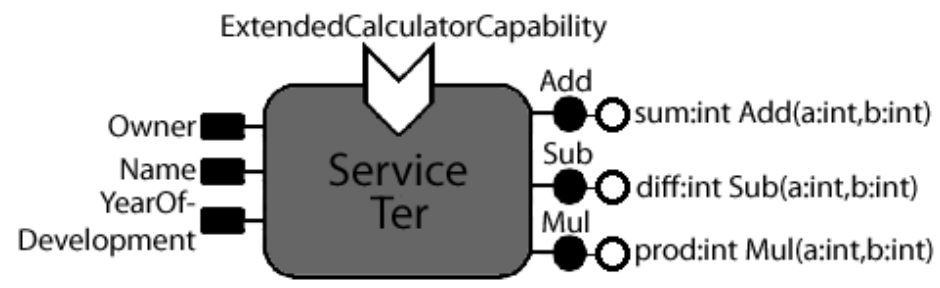

(d)

Figure A.1: Node type and service templates employed in testing the proof-of-concept implementation. 


\section{A.1 Implementation of the needed input}

This section will show how to represent the considered services (Figure A.1) in JAVA.

\section{Implementation of basic features}

Looking at the problem definition, we observe that (in order to represent the desired services) we need to develop some basic features.

First, we need to implement the required CalculatorCapabilityType (Listing A.1) and ExtendedCalculatorCapabilityType (Listing A.2). To do it, we have to suitably extend the generic Capability Type class.

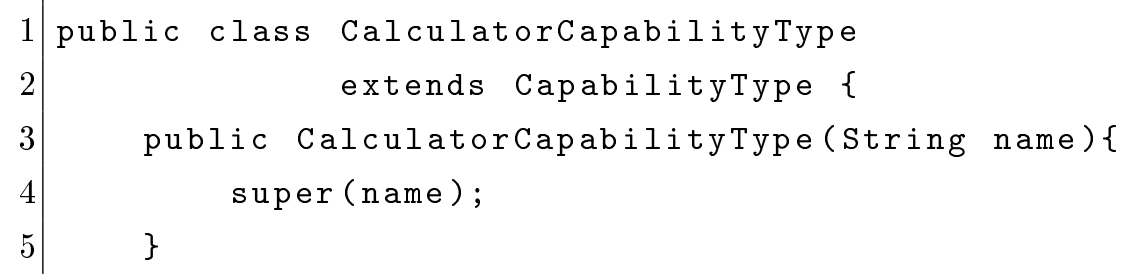

Listing A.1: JAVA implementation of the CalculatorCapabilityType.

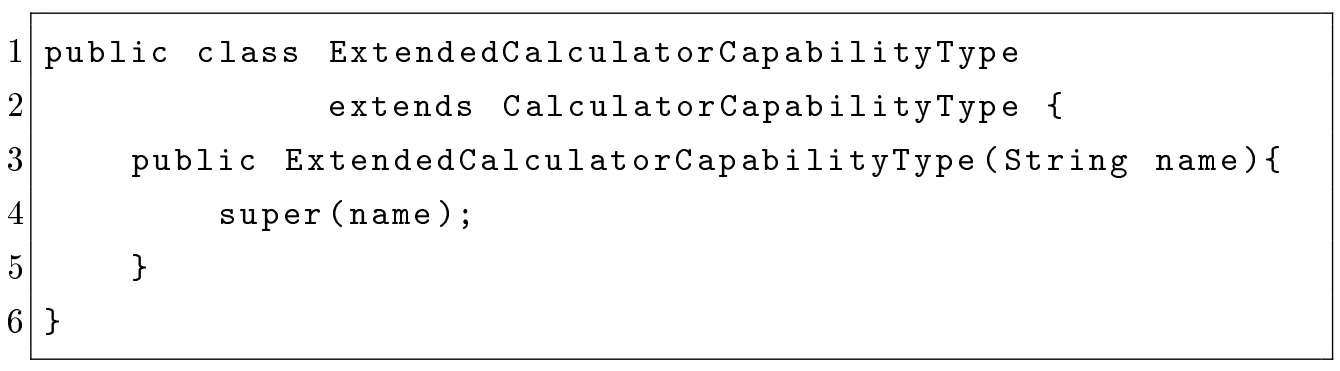

Listing A.2: JAVA implementation of the ExtendedCalculatorCapabilityType.

Then, we have to implement the type of the policy ${ }^{1}$ exposed by all services (Listing A.3).

\footnotetext{
${ }^{1}$ Please recall that such a policy type is assumed to be applicable to the CalculatorNodeType.
} 
Appendix A. Example of test of the proof-of-concept implementation

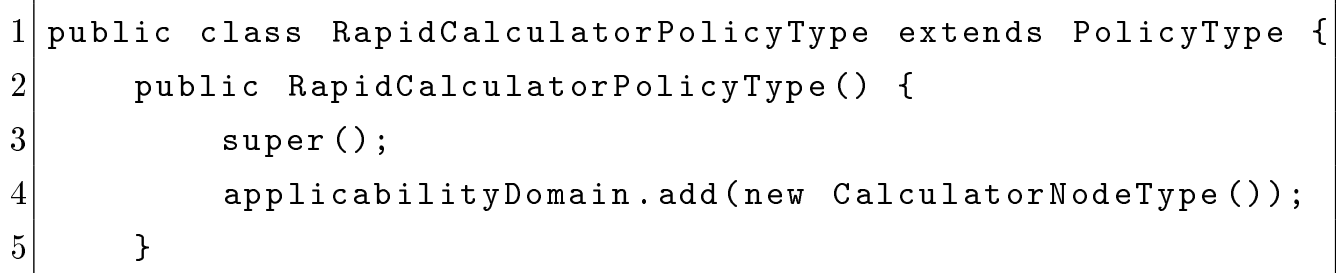

Listing A.3: JAVA implementation of the RapidCalculatorPolicyType.

Now, we have all the ground needed to implement the desired node type and service templates.

\section{Implementation of CalculatorNodeType}

The JAVA source code which implements the CalculatorNodeType is shown in Listing A.4.

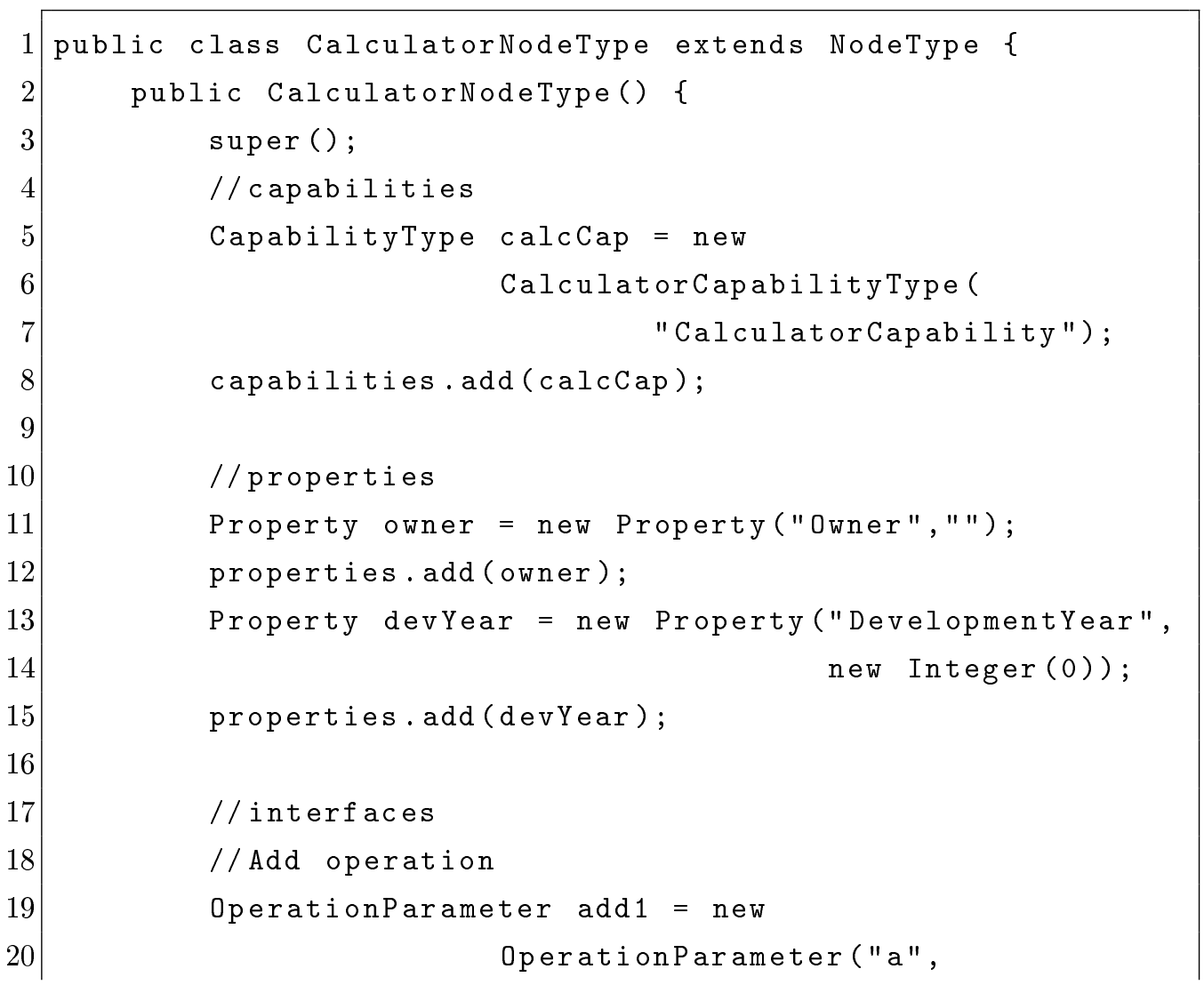


Appendix A. Example of test of the proof-of-concept implementation

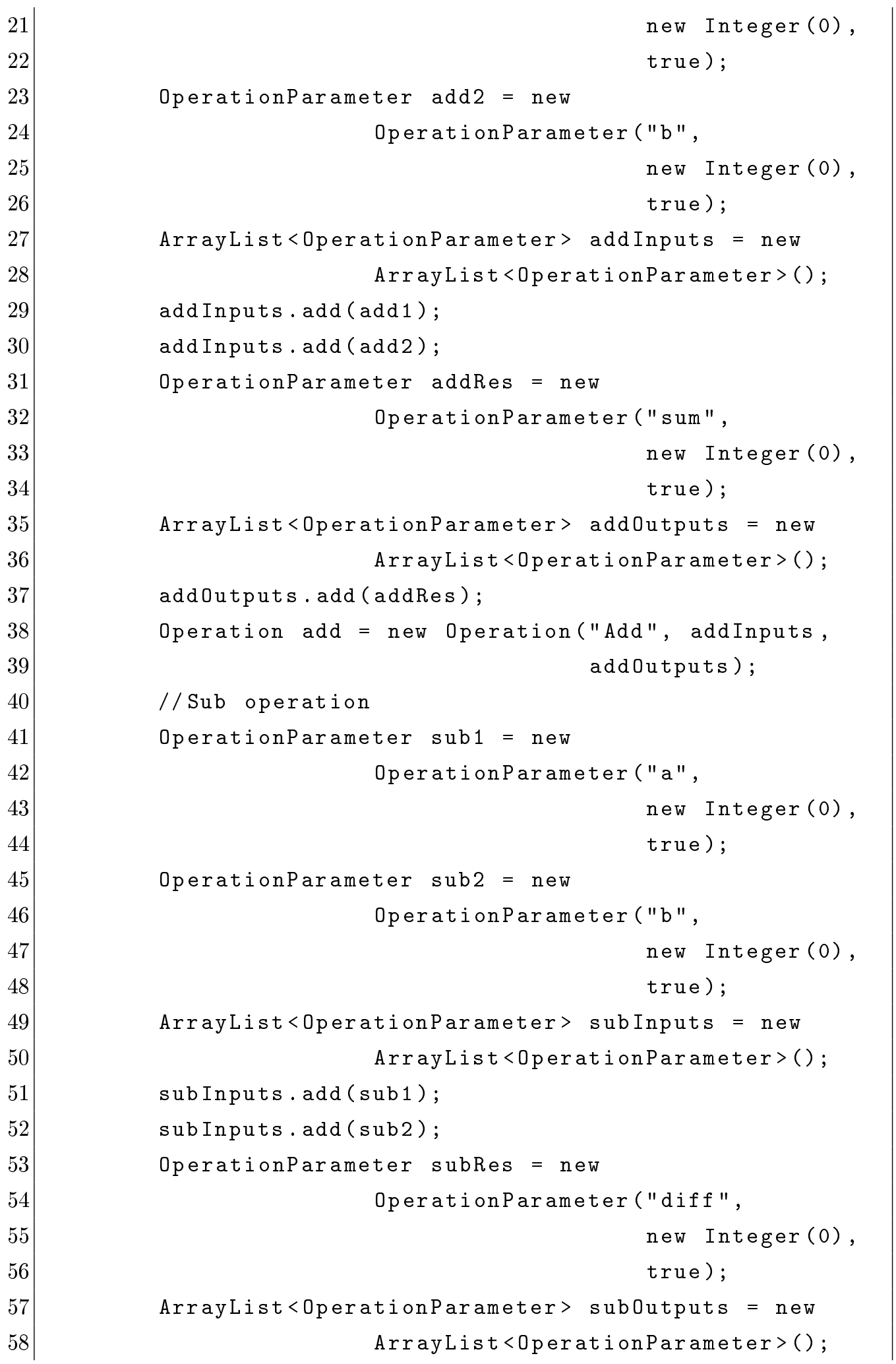




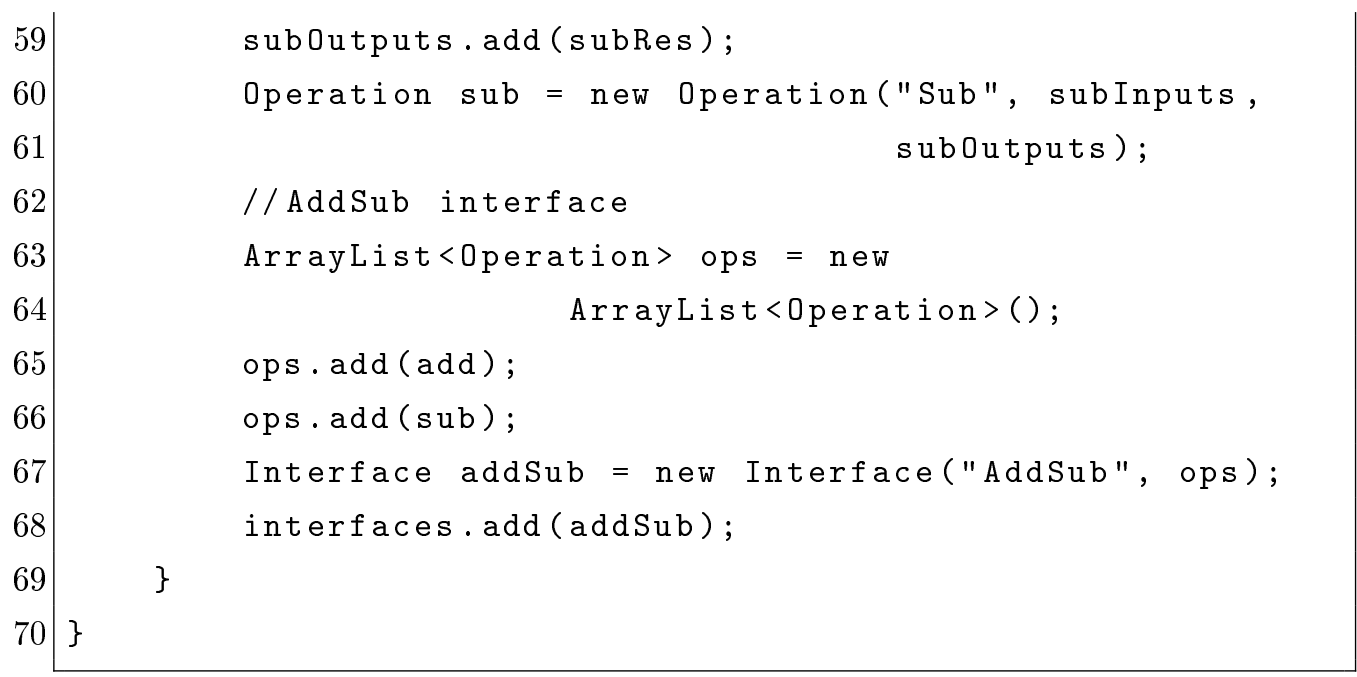

Listing A.4: JAVA implementation of the CalculatorNodeType.

\section{Implementation of Service}

The available Service is implemented in JAVA as shown in Listing A.5.

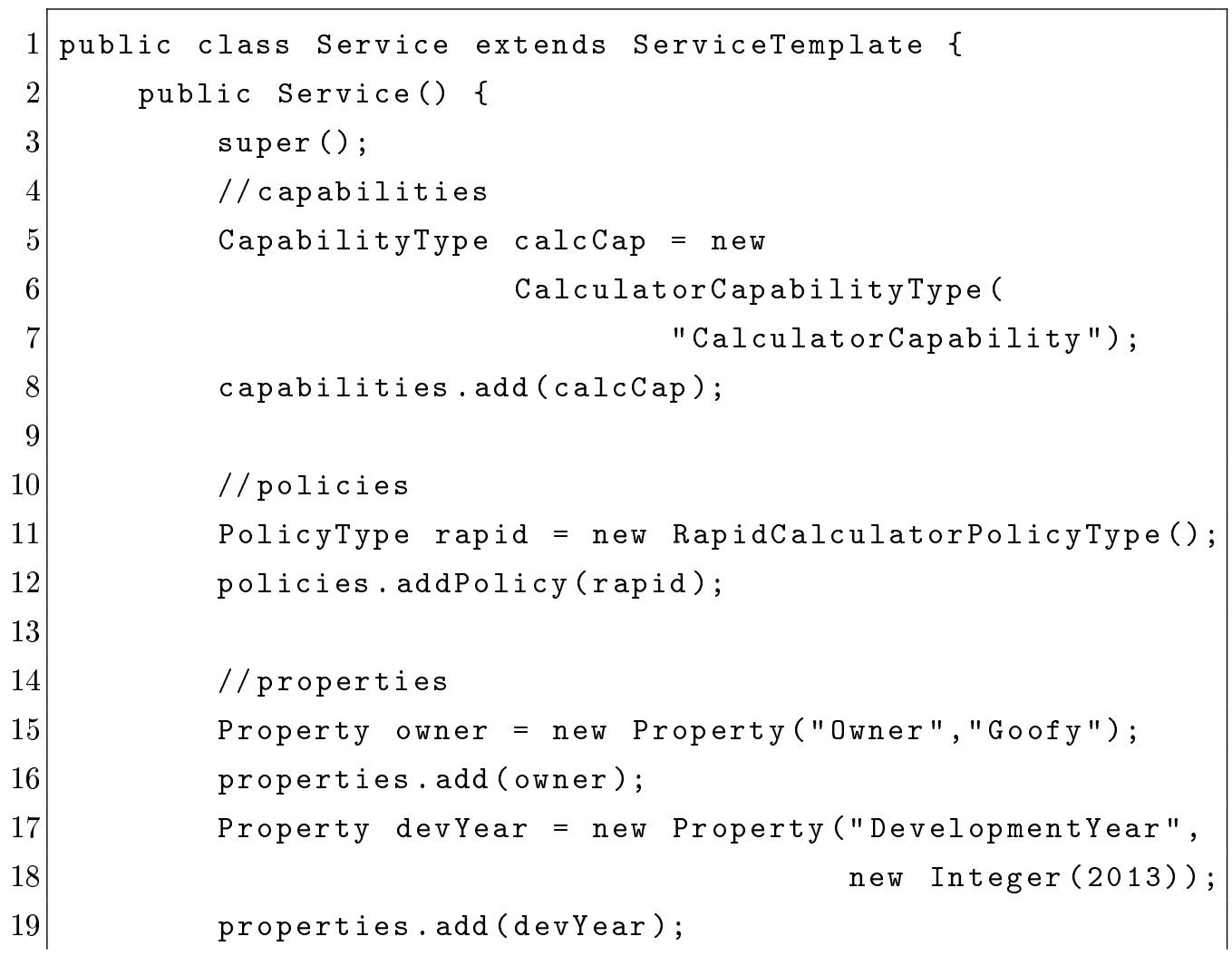




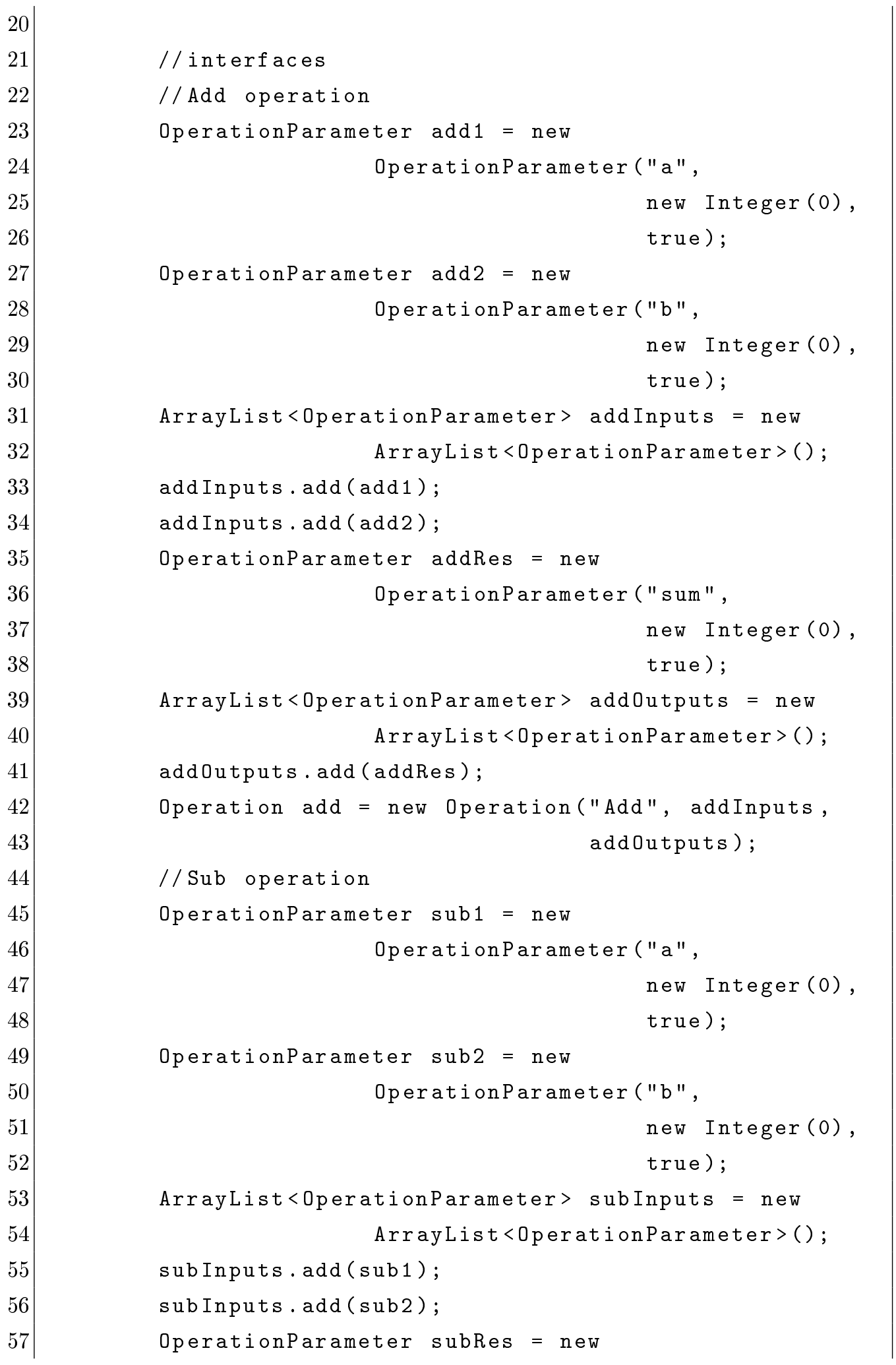


Appendix A. Example of test of the proof-of-concept implementation

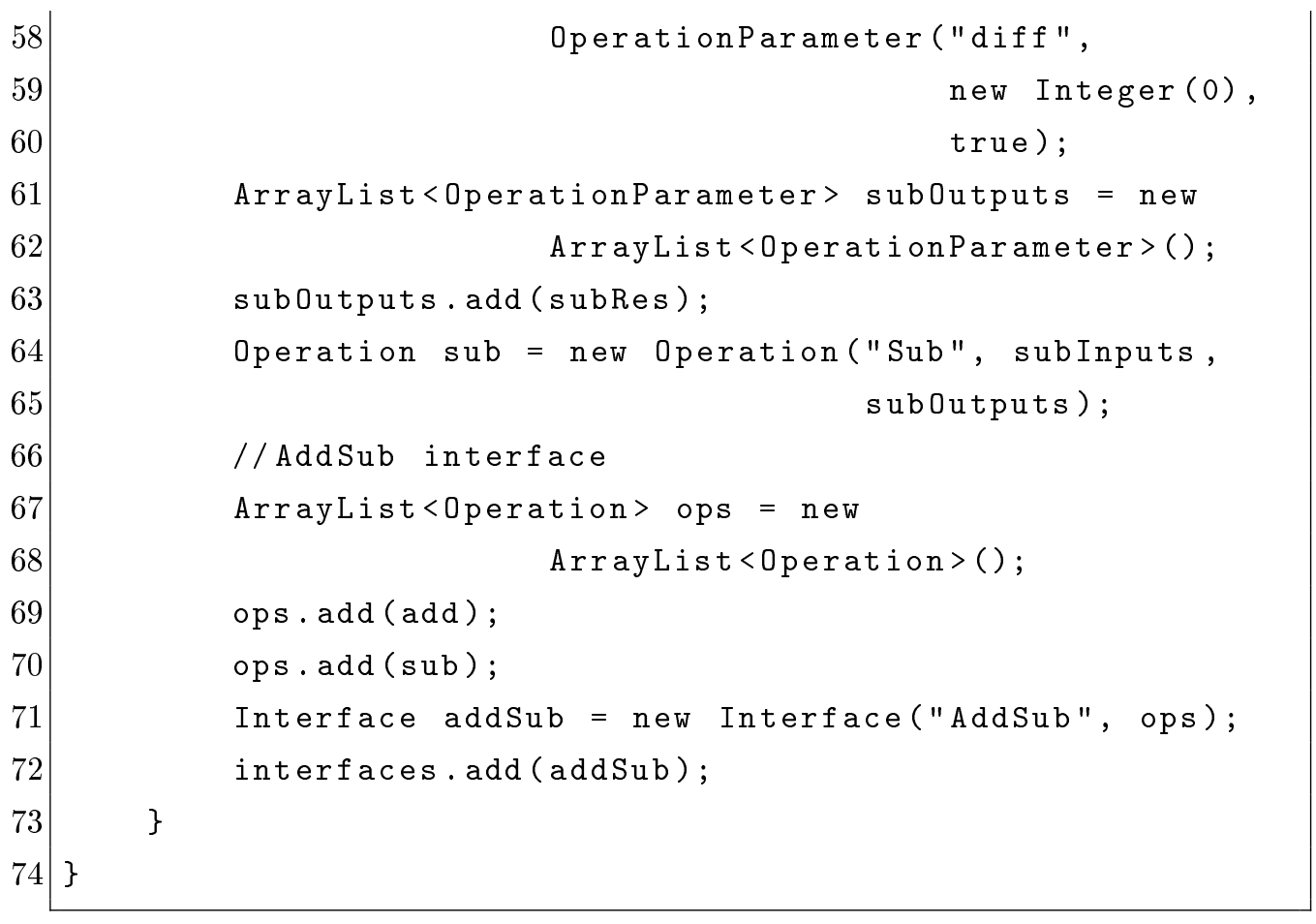

Listing A.5: JAVA implementation of Service.

\section{Implementation of ServiceBis}

Looking at Figure A.1, we observe that ServiceBis differs from Service since:

- it exposes a capability whose type is derived from that of the capability of Service,

- it exhibits an additional Name property, and

- it splits the $A d d S u b$ interface into two distinct interfaces and adds a new operation $M u l$ (contained in a homonym interface).

So, if we (suitably) modify the Service's source code, then we obtain ServiceBis (Listing A.6). 
Appendix A. Example of test of the proof-of-concept implementation

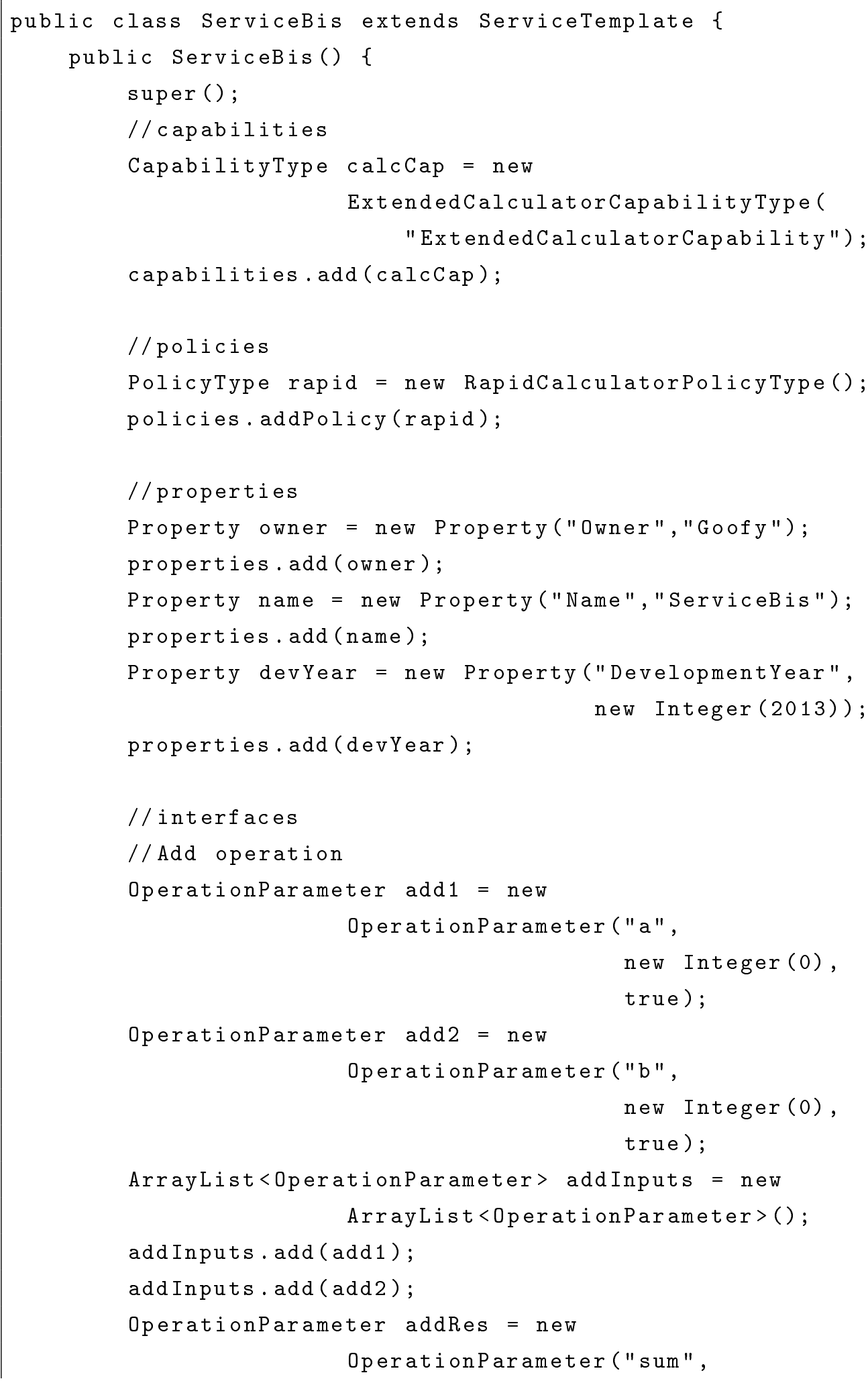


Appendix A. Example of test of the proof-of-concept implementation

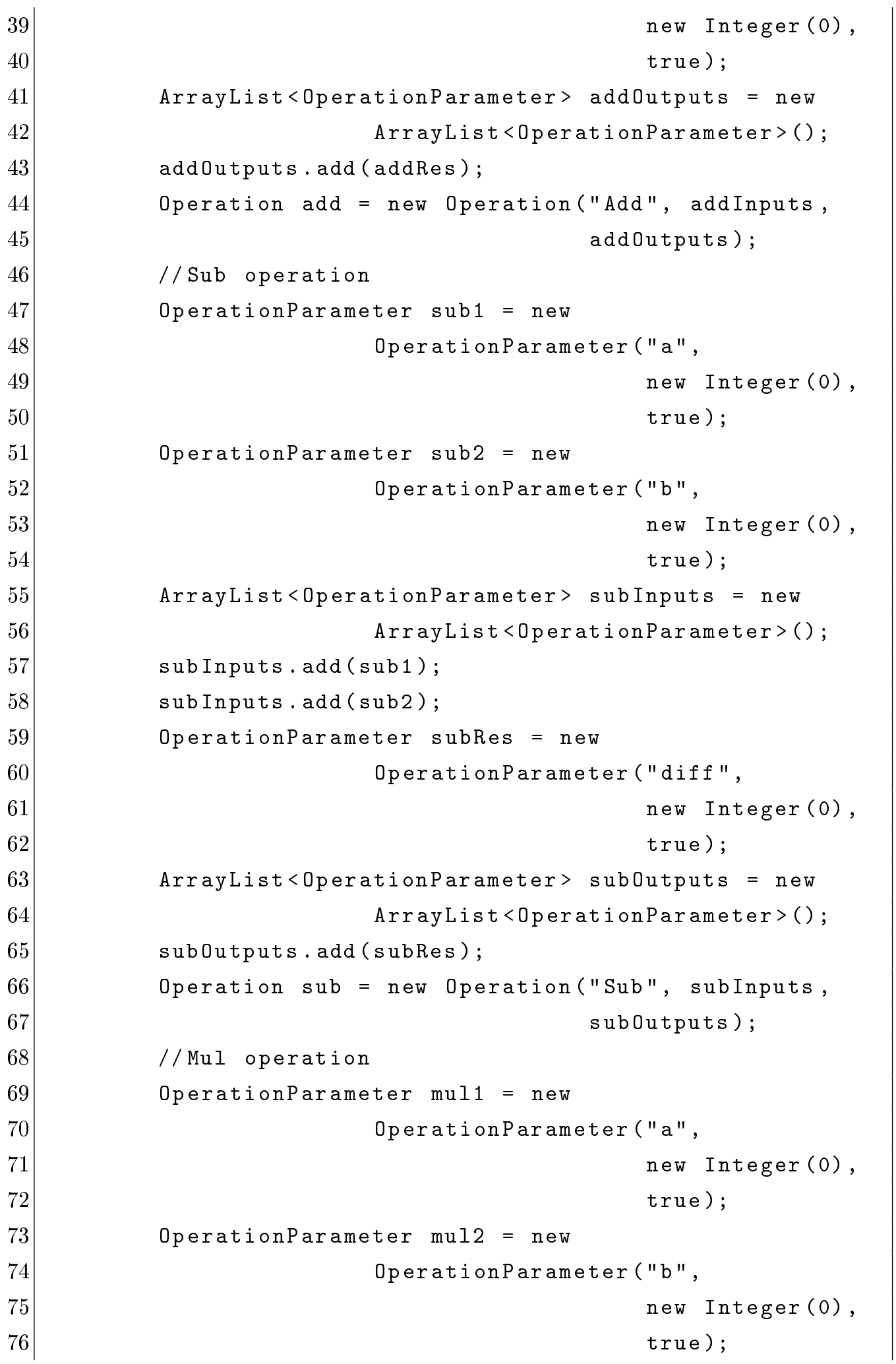


Appendix A. Example of test of the proof-of-concept implementation

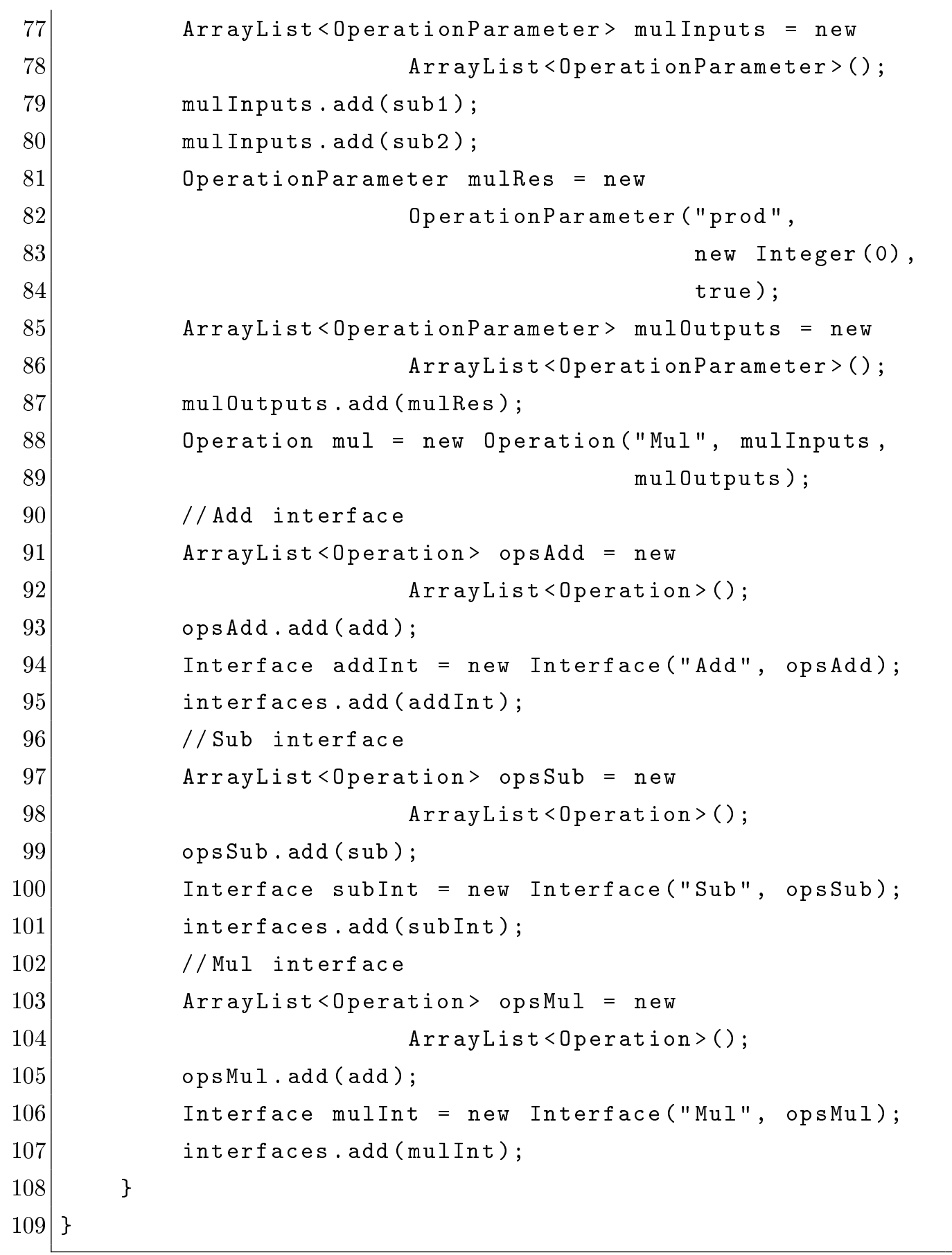

Listing A.6: JAVA implementation of ServiceBis. 


\section{Implementation of ServiceTer}

Looking at Figure A.1, we observe that ServiceTer differs from ServiceBis since it exposes the property YearOfDevelopment instead of the DevelopmentYear one. So, the source code of ServiceTer (Listing A.7) can be simply obtained by opportunely modifying line 19 of ServiceBis source code.

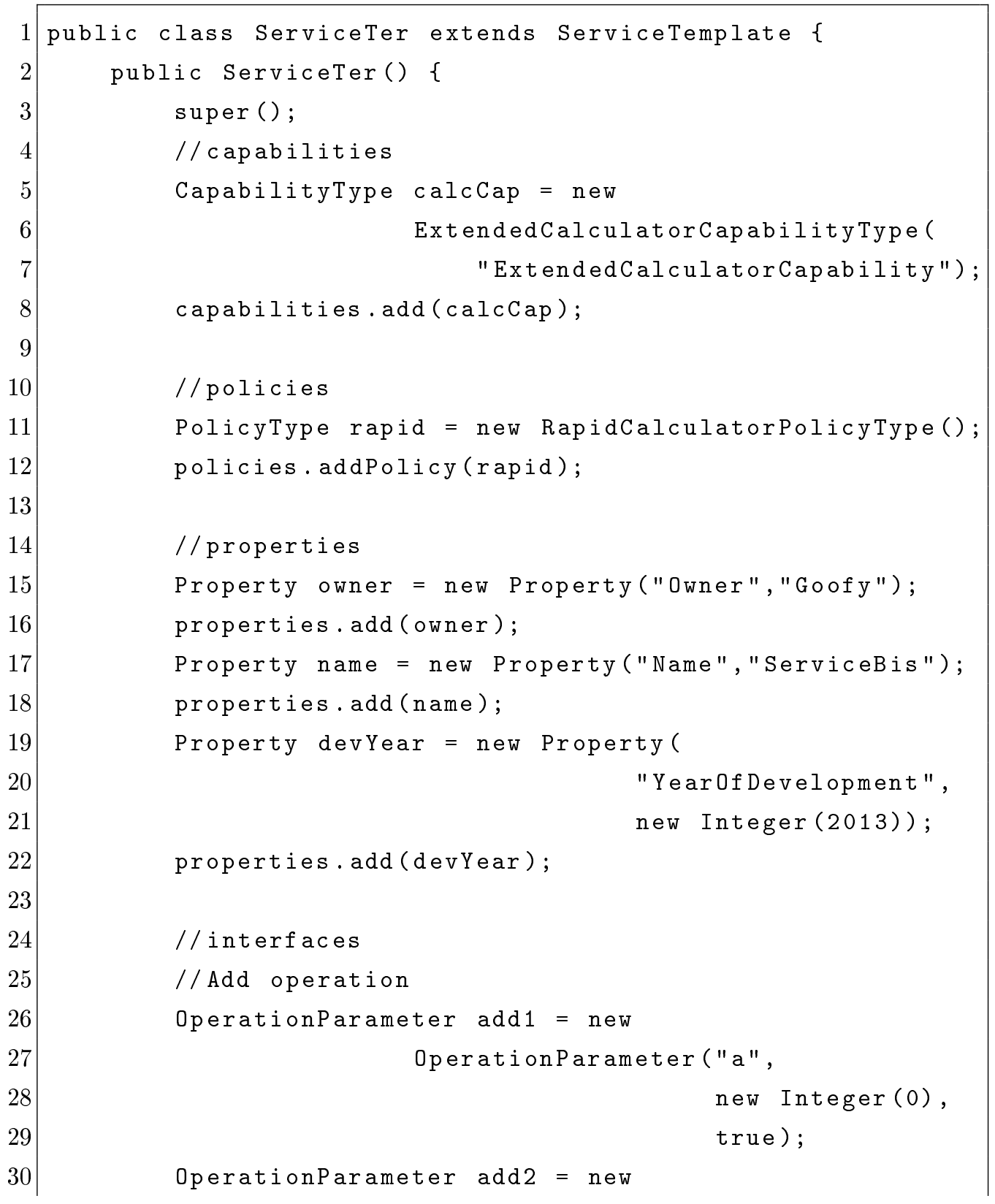


Appendix A. Example of test of the proof-of-concept implementation

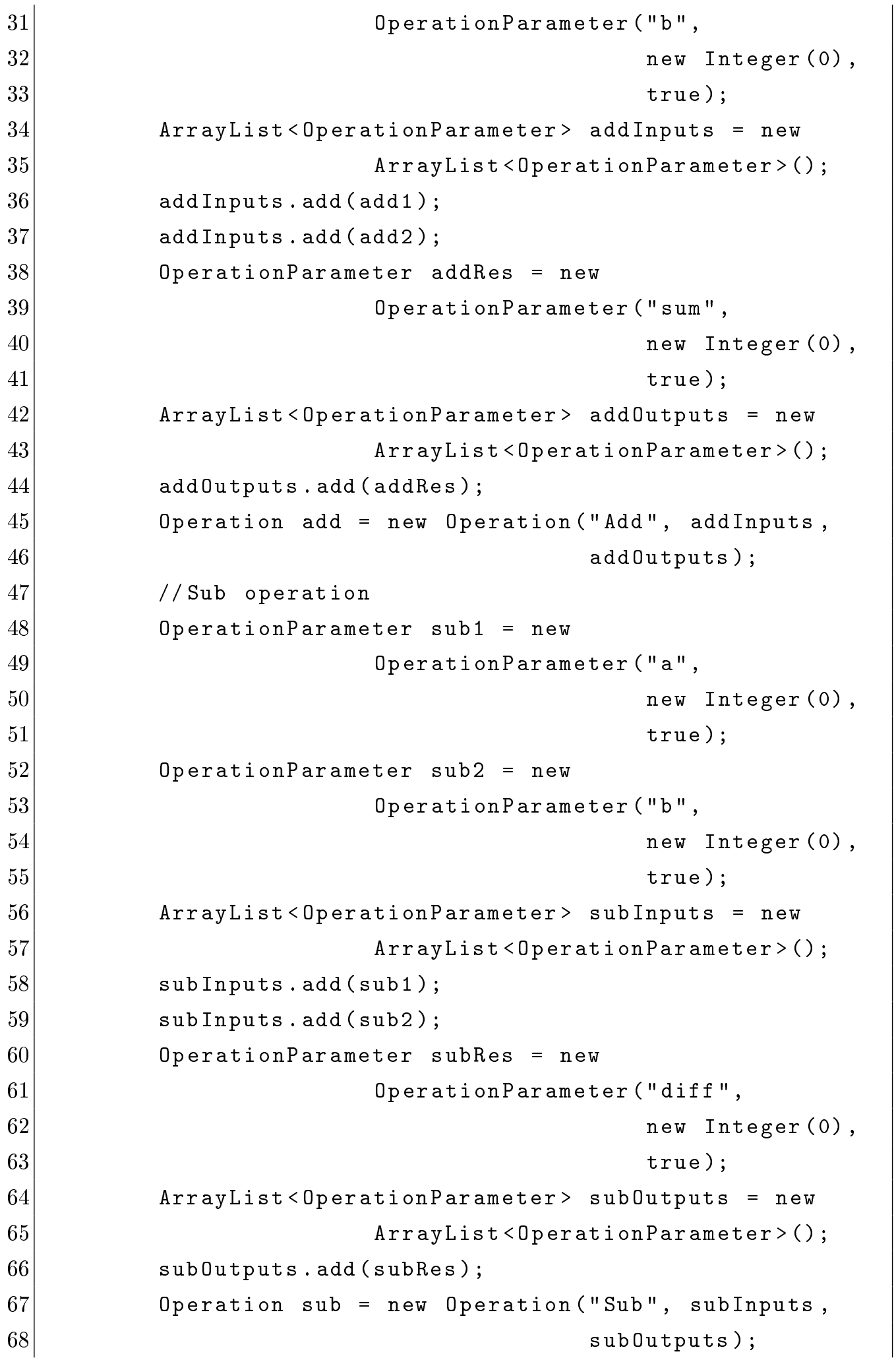




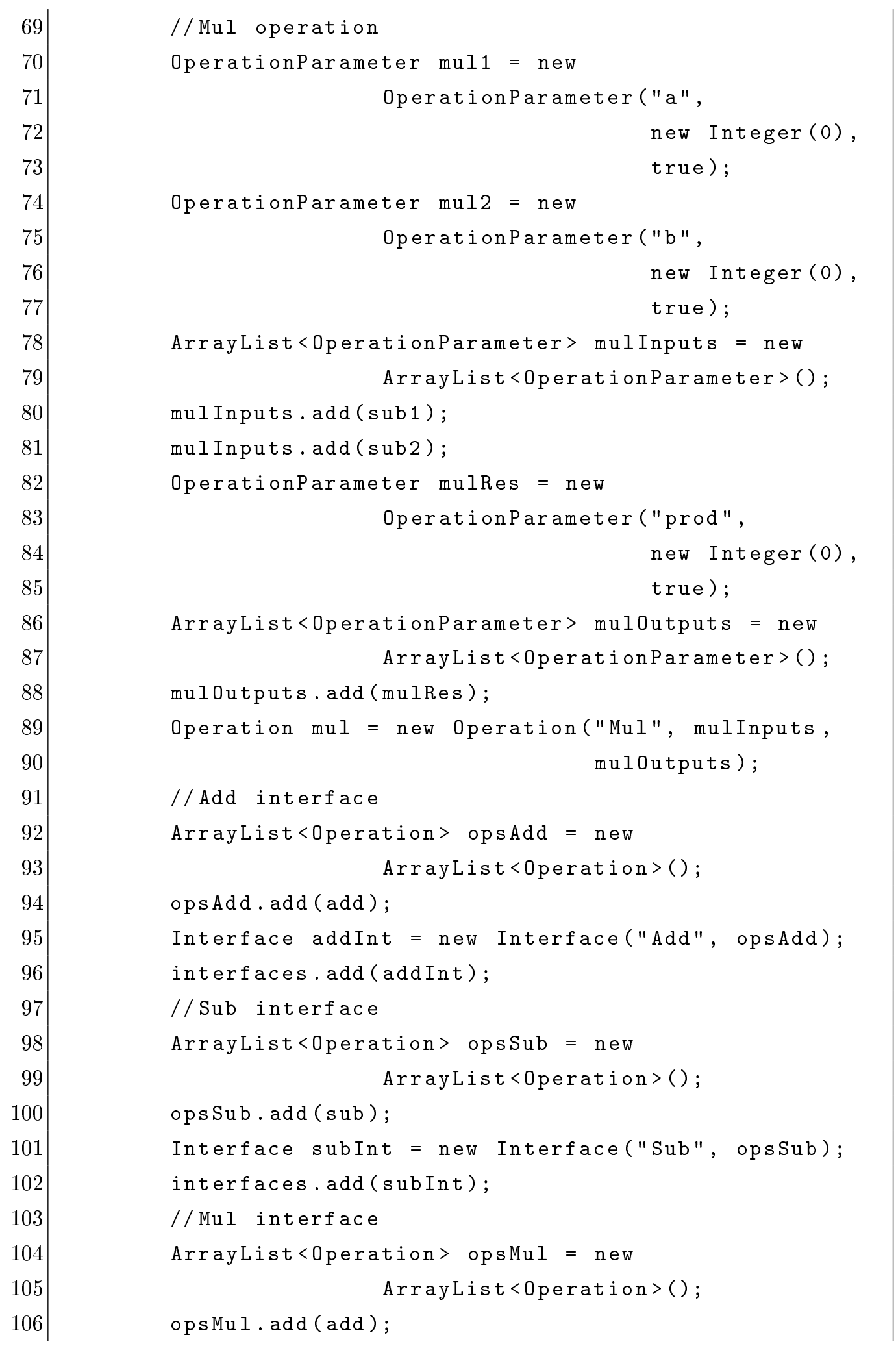


Appendix A. Example of test of the proof-of-concept implementation

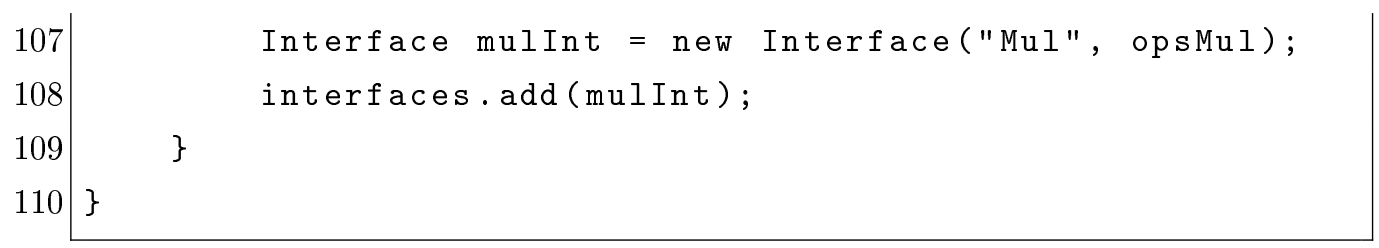

Listing A.7: JAVA implementation of ServiceTer.

\section{A.2 Implementation of the Test}

As mentioned at the very beginning of this Appendix $\mathrm{A}$, we want to show that the following conditions hold:

$$
\begin{gathered}
\text { CalculatorNodeType } \equiv \text { Service }(1), \\
\text { CalculatorNodeType } \neq \text { ServiceBis }(2), \\
\text { CalculatorNodeType } \subseteq \text { ServiceBis (3), and } \\
\text { CalculatorNodeType } \nsubseteq \text { ServiceTer (4). }
\end{gathered}
$$

To do it, we developed the runnable Test class (Listing A.8).

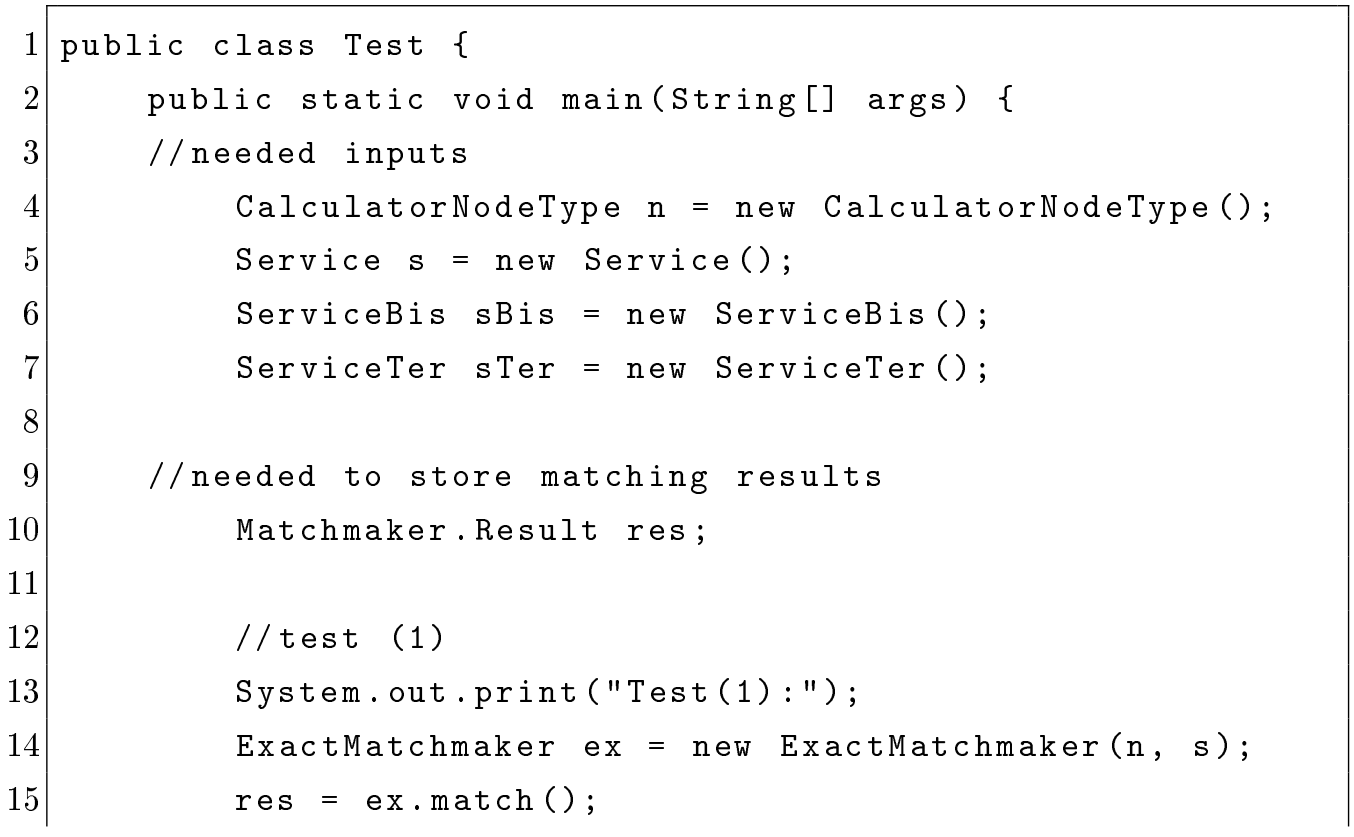


Appendix A. Example of test of the proof-of-concept implementation

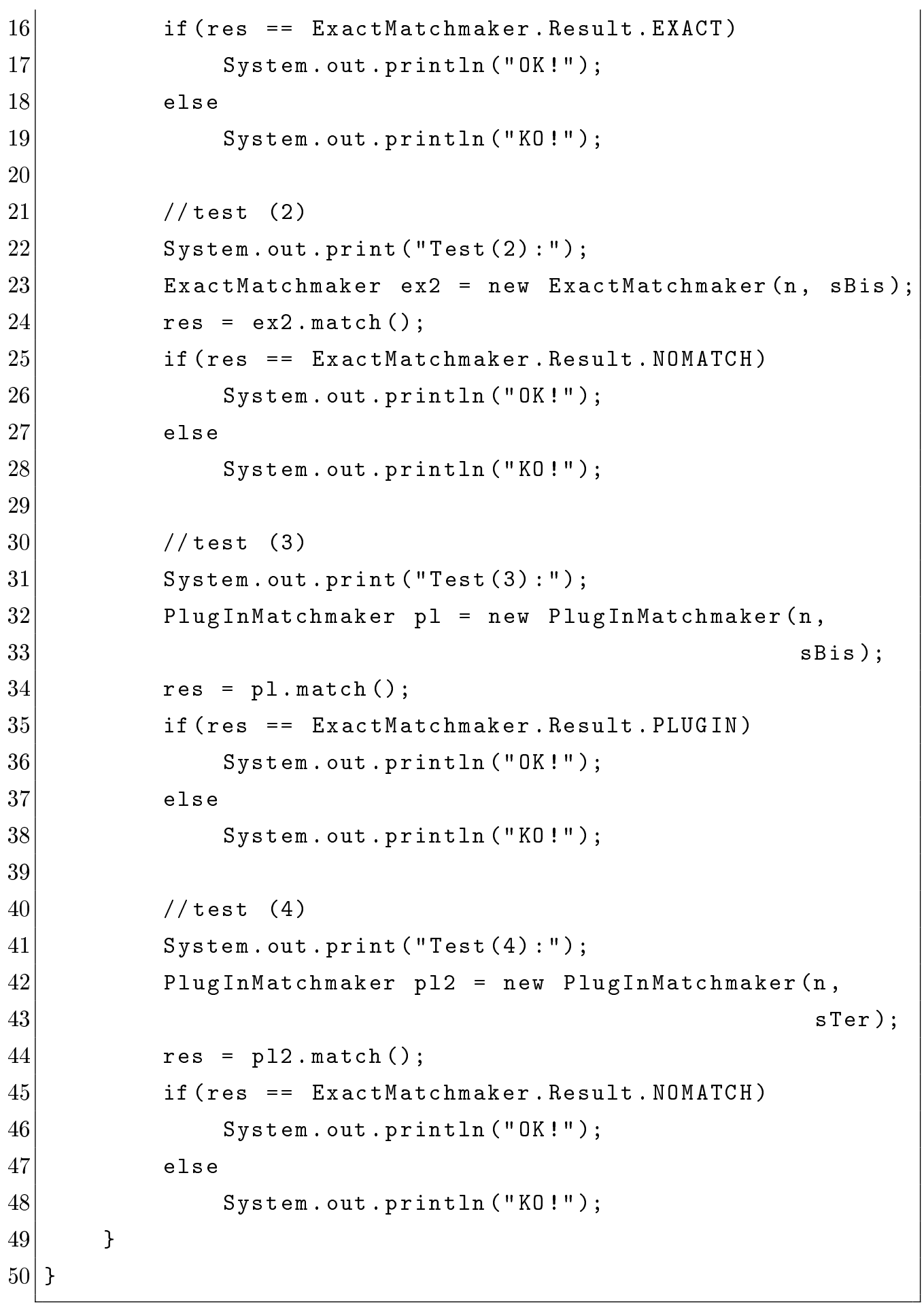

Listing A.8: JAVA implementation of the Test. 


\section{A.3 Concluding remarks}

Running the presented Test JAVA code we obtain what shown in Figure A.2. So, with this Appendix A, we have shown how the provided

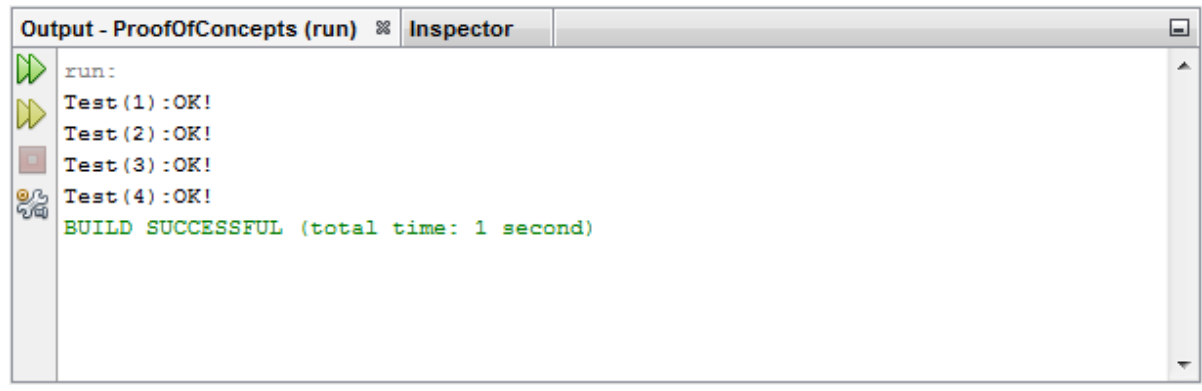

Figure A.2: Test results.

implementation (despite it is partial) let us perform some comparison tests. 


\section{Bibliography}

[1] M. Armbrust, A. Fox, R. Griffith, A.D. Joseph, R. Katz, A. Konwinski, G. Lee, D. Patterson, A. Rabkin, I. Stoica, M. Zaharia, A View of Cloud Computing, Communications of the ACM, vol. 53, issue 4, pages 50-58, April 2010, doi:10.1145/1721654.1721672;

[2] T. Binz, G. Breiter, F. Leyman, T. Spatzier, Portable Cloud Services Using TOSCA, IEEE Internet Computing, vol. 16, no. 3, pp. 80-85, May-June 2012, doi:10.1109/MIC.2012.43;

[3] F. Bonchi, A. Brogi, S. Corfini, F. Gadducci, A Net-based Approach to Web Services Publication and Replaceability, Fundamenta informaticae, 94(3-4):205-309, 2009.

[4] A. Brogi, Service Adaptation, ESOCC'12, Bertinoro, September 2012 ;

[5] A. Brogi, S. Corfini, Behaviour-aware discovery of Web service compositions, International Journal of Web Service Research, vol. 4, issue 3, July 2007;

[6] A. Brogi, S. Corfini, J. F. Aldana, I. Navas, Automated Discovery of Compositions of Services Described with Separate Ontologies, Proceedings of the 4th International Conference on Service Oriented Computing (ICSOC 06), LNCS vol. 4294, pages 509-514, 2006;

[7] A. Brogi, S. Corfini, J.F. Aldana, I. Navas, A Prototype for Discovering Compositions of Semantic Web Services, Proceedings of the 
Third Italian Workshop on Semantic Web Applications and Perspectives, 2006;

[8] A. Brogi, S. Corfini, R. Popescu, Semantics-Based CompositionOriented Discovery of Web Services, ACM Transaction on Internet Technology, vol.8, no. 4, article 19, September 2008;

[9] A. Brogi, R. Popescu, Service Adaptation through Trace Inspection, International Journal of Business Process Integration and Management, vol. 2, no. 1, pp. 9-16 (8), June 2007;

[10] A. Brogi, R. Popescu, M. Tanca, Design and implementation of SATOR: A Web service aggregator, ACM Transactions on Software Engineering and Methodology, 19(3), 2010;

[11] S. Corfini, Composition-oriented Web Service Discovery, Lightning Source Incorporated, isbn:9783639041569, 2008;

[12] J. C. Corrales, D. Grigori, M. Bouzeghoub, BPEL processes matchmaking for service discovery, Proceeding ODBASE'06/OTM'06 Proceedings of the 2006 Confederated international conference on On the Move to Meaningful Internet Systems: CoopIS, DOA, GADA, and ODBASE - Volume Part I, pp. 237-254, doi:10.1007/1191485315, 2006;

[13] F. Eliassen, S. Mehus, Type Checking Stream Flow Endpoints, Middleware '98, Springer London, pp. 305-320, doi:10.1007/978-1-44711283-9_19, 1998;

[14] R. Eshuis, P. Grefen, Structural Matching of BPEL Processes, Proceeding ECOWS '07 Proceedings of the Fifth European Conference on Web Services, pp. 171-180 , doi:10.1109/ECOWS.2007.26, 2007;

[15] G. Gallo, G. Longo, S. Nguyen, S. Pallottino, Directed Hypergraphs and Applications, Discrete Applied Mathematics, 42(2):177-201, 1993 ; 
[16] M. Klusch, B. Fries, K. Sycara, Journal, OWLS-MX: A hybrid Semantic Web service matchmaker for OWL-S services, Web Semantics: Science, Services and Agents on the World Wide Web archive, vol. 7, issue 2, pp 121-133, doi:10.1016/j.websem.2008.10.001, April 2009 ;

[17] Q. Hardy, Active in Cloud, Amazon Reshapes Computing, The New York Times, 27 August 2012, http://www.nytimes.com/2012/08/28/technology/ active-in-cloud-amazon-reshapes-computing.html;

[18] P. Lipton, Escaping Vendor Lock-In with TOSCA, an Emerging Cloud Standard for Portability, CA Technology Exchange (CA Labs Research), CA Technologies, vol.4, issue 1, pp. 49-55, January 2013;

[19] F. Mahdikhani, M.R. Hashemi, M. Sirjani, QoS Aspects in Web Services Compositions, Proceedings of IEEE International Symposium on Service-Oriented System Engineering SOSE '08., pp. 239-244, doi:10.1109/SOSE.2008.39, 2008;

[20] J. Martinez-Gil, I. Navas-Delgado, J.F. Aldana-Montes, MaF: An Ontology Matching Framework, Journal of Universal Computer Science, vol. 18, no. 3, pp. 194-217, January 2012;

[21] P. Mell, T. Grance, The NIST Definition of Cloud Computing, NIST Special Publication 800-145, September 2011, http://csrc.nist. gov/publications/nistpubs/800-145/SP800-145.pdf;

[22] J. Miranda, J. Guillen, J.M. Murillo, C. Canal, Development of Adaptive Multi-cloud Applications: A Model-Driven Approach, MODELSWARD, 2013;

[23] S.B. Mokhtar, D. Preuveneers, N. Georgantas, V. Issarny, Y. Berbers, EASY: Efficient semAntic Service discover $Y$ in pervasive computing environments with QoS and context support, J. Syst. 
Softw., vol. 81, no. 5, pp. 785-808, doi: 10.1016/j.jss.2007.07.030, Elsevier Science Inc., May 2008;

[24] P. Muschamp, An introduction to Web Services, BT Technology Journal, vol. 22, no. 1, January 2004;

[25] OASIS TOSCA TC, Topology and Orchestration Specification for Cloud Applications Version 1.0, http://docs.oasis-open.org/ tosca/TOSCA/v1.0/TOSCA-v1.0.pdf;

[26] OASIS TOSCA TC, Topology and Orchestration Specification for Cloud Applications (TOSCA) Primer Version 1.0, http://docs.oasis-open.org/tosca/tosca-primer/v1.0/ tosca-primer-v1.0.pdf

[27] OpenTOSCA, http://www.iaas.uni-stuttgart.de/OpenTOSCA/ indexE.php;

[28] D. O'Sullivan, D. Lewis, Semantically driven service interoperability for pervasive computing, Proceedings of the 3rd ACM international workshop on Data engineering for wireless and mobile access, pp. 17-24, doi:10.1145/940923.940927, 2003;

[29] D. Petcu, G. Macariu, S. Panica, C. Craciun, Portable Cloud Applications - From Theory to Practice, Future Generation Computer Systems, vol. 29, issue 6, pp. 1417-1430, 2012;

[30] L. Schubert, K. Jeffery (Eds.), Advances in Clouds, Expert Group Report, Public version 1.0, European Commission, http://ec.europa.eu/information_society/newsroom/cf/ /document.cfm?doc_id=1174, 2012;

[31] S. Tummalapalli, P. RaviKanth, K. Yuvaraj, S. Velagapudi, TOSCA Enabling Cloud Portability, International Journal of Advanced Research and Computer Engineering \& Technology, vol. 2, no. 3, 2013;

[32] Valesca, http://www.cloudcycle.org/en/valesca/; 
[33] A. Weiss, Master Thesis: Merging of TOSCA Cloud Topology Templates, Institute of Architecture of Application Systems, University of Stuttgart, http://elib.uni-stuttgart.de/opus/volltexte/ 2012/7932/pdf/MSTR_3341.pdf;

[34] G.J. Woeginger, Zhongliang Yu, On the equal-subset-sum problem, Information Processing Letters, vol. 42, no. 6, pp. 299-302, 1992;

[35] W3C, Extensible Markup Language (XML) 1.0 (Fifth Edition), 26 November 2008, http://www.w3.org/TR/xml

[36] W3C, XML Path Language (XPath) Version 1.0, 16 November 1999, http: //www.w3.org/TR/xpath/;

[37] W3C, XML Schema Part 0: Primer Second Edition, 28 October 2004, http: //www .w3.org/TR/xmlschema-0 


\section{List of Abbreviations}

IT

OASIS

TOSCA

URI

URL

W3C

XML

XPath
Information Technology

Organization for the Advancement of Structured Information Standards

Topology and Orchestration Specification for Cloud Applications

Universal Resource Identifier

Universal Resource Locator

World Wide Web Consortium

eXtensible Markup Language

XML Path Language 


\section{Index}

OperationSETSDiscovering, 90 functional equivalence $(\Leftarrow), 88$ completeness, 98

complexity, 100

soundness, 96

hyperedge, 83

directed, 84

labelled directed, 85

cross-ontology matchmaker, 66

hypergraph, 83

dependencies (functional), 82

dependency, 84

inter-operation, 82,85

intra-operation, 82,85

derived-from checking operator $(\vdash)$,

49

minimality (of Operation sets), 92 .

93

exact matching (三), 32

construction, 86

directed, 84

labelled directed, 84

capabilities, 34

examples, 44

oblivion boundaries, 53

extension, 66

interfaces, 43

operations, 43

policies, 37

properties, 39

requirements, 34

flexible matching $(\cong), 62$

assumptions, 63

example, 66

plug-in matching $(\subseteq), 48$

capabilities, 50

example, 57

interfaces, 52

properties, 51

requirements, 50

policies applicability operator $(\succ)$,

interfaces, 65

operations, 65

properties, 64

36

sub-concept of, 86

hypergraph extension, 86

operator $(\triangleleft), 88$ 
TOSCA, 6

BoundaryDefinitions, 13

CapabilityType, 17

Definitions, 9

Node Type, 14

Plans, 14

PolicyTemplate, 20

Policy Type, 19

Relationship Type, 16

RequirementType, 17

Service Template, 11

Topology Template, 14

use cases, 7

weather forecast example, 21

white-box matching (@), 75

capabilities, 77

interfaces, 79

properties, 78 